\title{
Nonlinearities in bipolar cells and their role for encoding visual signals
}

\author{
Dissertation \\ for the award of the degree \\ "Doctor rerum naturalium" \\ of the Georg-August-Universität Göttingen \\ within the doctoral program \\ Sensory and Motor Neuroscience \\ of the Georg-August University School of Science (GAUSS)
}

submitted by

Helene Marianne Schreyer

from: Bern, Switzerland

Göttingen, 2018 


\section{Thesis committee}

First referee and supervisor

Prof. Dr. Tim Gollisch

Sensory Processing in the Retina, Department of Ophthalmology,

University Medical Center Göttingen

Second referee

Prof. Dr. Jochen Staiger

Center of Anatomy, Department of Neuroanatomy,

University Medical Center Göttingen

Third referee

Prof. Dr. Jeong Seop Rhee

Neurophysiology Group

Max Planck Institute for Experimental Medicine, Göttingen

\section{Further members of the examination board}

Dr. Marion Silies

Visual Processing group

European Neuroscience Institute Göttingen (ENI)

Prof. Dr. Tobias Moser

Institute for Auditory Neuroscience \& Inner Ear Lab

University Medical Center Göttingen

Prof. Dr. André Fiala

Schwann-Schleiden Research Centre

Department of Molecular Neurobiology of Behavior

University of Göttingen

Date of oral examination: 8 May 2018 


\section{Declaration}

I hereby declare that this thesis entitled "Nonlinearities in bipolar cells and their role for encoding visual signals" is my own work and no other source than quoted were used.

Helene Marianne Schreyer

Göttingen, March 2018 

Ich bin der Sucher also suche ich die Suche. in Erinnerung an Bob, irgendwo im Eve 



\section{Acknowledgements}

First, I would like to thank my advisor Tim Gollisch for giving me the chance to write my $\mathrm{PhD}$ in his lab. I am grateful for his continuous support, our discussions, his patience in explaining mathematical concepts and his great knowledge. I learned many things from Tim and I appreciate his exactness and fascination for science.

Besides my advisor, I would like to thank the members of my thesis committee, Jochen Staiger and Jeong Seop Rhee. I appreciated that they shared their knowledge with me and I enjoyed my thesis committee meetings, the questions and discussions. I also like to thank the members of the extended examination board Marion Silies, Tobias Moser and André Fiala for evaluating my defense.

Further, I would like to thank Jochen Staiger, Patricia Sprych and Mirko Witte for sharing with me the procedures of staining individual neurons with neurobiotin. Patricia Sprych was very patient and I enjoyed the days I spent with her at the lab bench.

My sincere thanks goes to Mohammad Hossein Khani and Michael Weick. Without their help in building my setup and their support in many technical issues my research would not be possible. Michael Weick taught me how to prepare retinas and shared many of his little tricks with me to reach beautiful recordings. Mohammad Hossain Khani supported me for the multielectrode array recordings and I could ask him any question.

I am very grateful to all my colleagues in our group "sensory processing in the retina". I appreciate our lunch breaks and "bakery" breaks. I enjoy our discussions about nonlinearities and other scientific and political problems. I enjoy the late nights, the weekends and dinners we spent together in the lab. I really like our cinema nights! Thank you Fernando Rozenblit, Norma Kühn, Dimokratis Karamanlis, Vidhyasankar Krishnamoorthy, Yunus Can Erol, Jian Liu, Diasuke Takeshita and Luis Giordano Ramos Traslosheros Lopez.

Last but not least, I would like to thank my "Schreyers": my parents, my sisters, my brother, my nephews and all other new and old family members. I am happy to be part of you! 



\section{Abstract}

Vision begins in the retina, where ganglion cells separate the visual input into $\sim 30$ parallel output channels with different response characteristics to visual stimuli. How retinal ganglion cells obtain such a diversity of functional properties is unclear. The diversity appears to evolve along the signal processing stream from photoreceptors to ganglion cells. Along this pathway, bipolar cells represent pivotal elements by connecting the photoreceptors, the horizontal cells and the amacrine cells to the retinal ganglion cells. Despite their crucial position, our knowledge about bipolar cells is limited. Furthermore, simplifying assumptions about their light responses are made. For example, it is broadly assumed that bipolar cells respond to light linearly.

In this thesis, we investigated the assumption of linear signal processing in bipolar cells. To do so, we worked on four main goals:

- Goal 1: Establishing a general characterization of bipolar cells

- Goal 2: Assessing nonlinearities in bipolar cells

- Goal 3: Predicting bipolar cells' responses with the linear-nonlinear model

- Goal 4: Simultaneous recordings from bipolar and ganglion cells

We investigated the goals in the salamander retina by recording the voltage signals of bipolar cells with single electrodes. We observed a diversity of bipolar cell responses to simple and complex light stimuli (goal 1). We observed nonlinear responses of bipolar cells in their contrast representation and in their input integration (goal 2). Further, mathematical models like the linearnonlinear model failed to predict responses of some bipolar cells to complex artificial and natural light stimuli (goal 3). Finally, the established method of simultaneous recordings from bipolar and ganglion cells was used to study the connection between bipolar cells and mathematically retrieved subunits in ganglion cells (goal 4). Taken together, our work suggests that nonlinear signal transformation starts at the level of the input integration in bipolar cells and that the bipolar cell nonlinearities have to be taken into consideration for mathematical encoding models in the retina. 



\section{Contents}

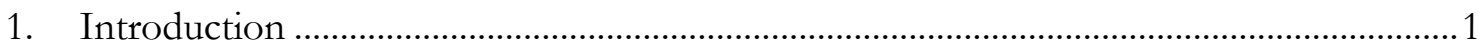

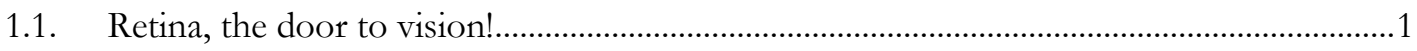

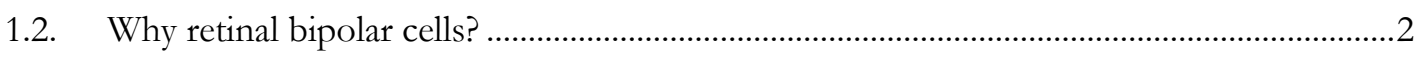

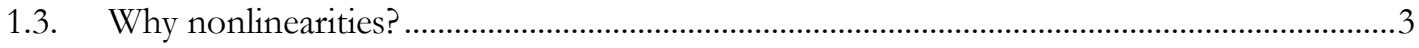

1.4. Nonlinearities: the burden for encoding!........................................................................

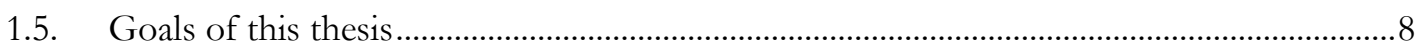

1.6. Outline of this thesis....................................................................................................

2. Background: Research in the vertebrate retina ............................................................11

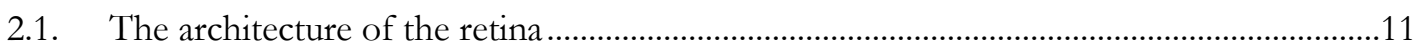

2.2. General functional characterization in the retina ................................................................18

2.3. The role of nonlinearities in the retina............................................................................23

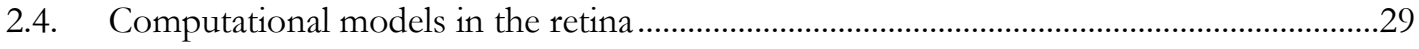

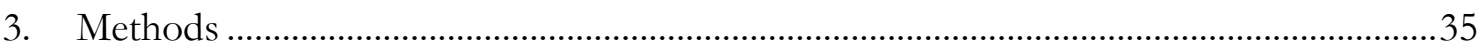

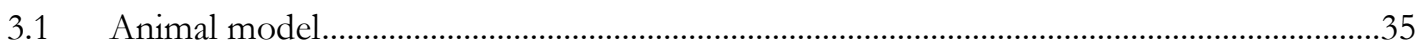

3.2 Tissue preparation ..........................................................................................................36

3.3 Simultaneous recordings from bipolar and ganglion cells ...................................................37

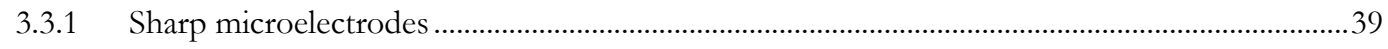

3.3.2 Multielectrode array ………………………………………………………………………………….... 40

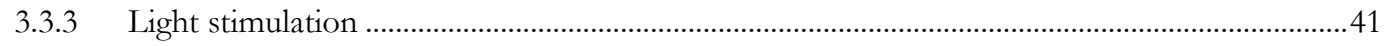

3.4 Identifying recorded cell types ............................................................................................

3.5 General response characterization..................................................................................4

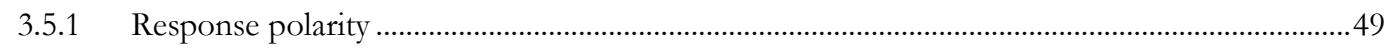

3.5.2 Receptive field center and surround estimation ..............................................................................51

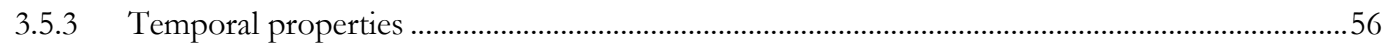

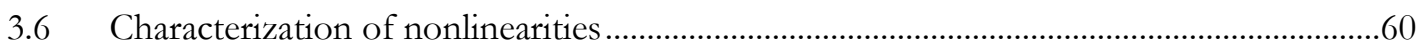

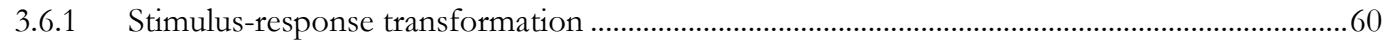

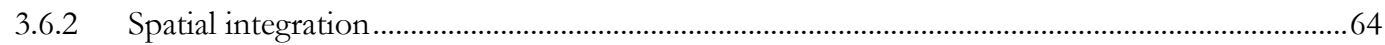

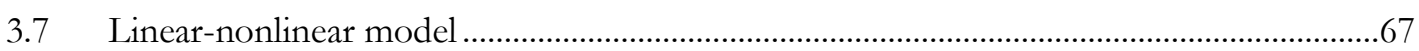




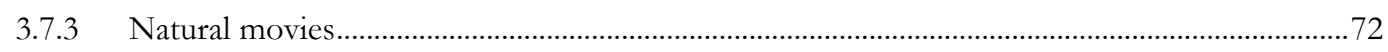

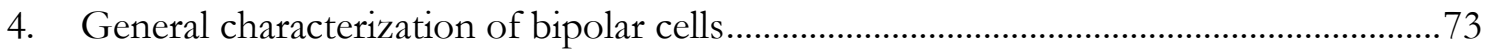

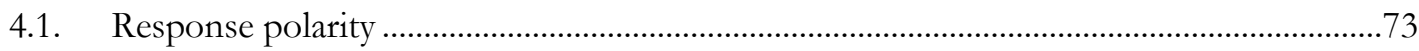

4.2. Receptive field center estimation ......................................................................................

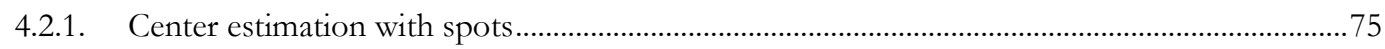

4.2.2. Center estimation with binary white noise ….............................................................................

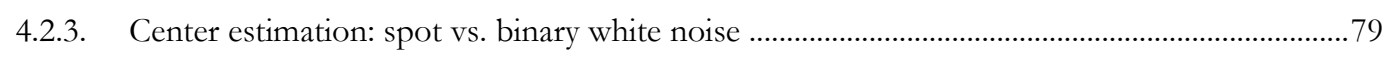

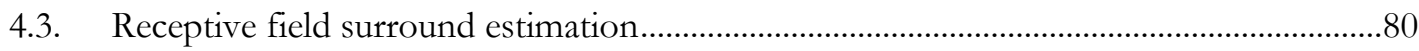

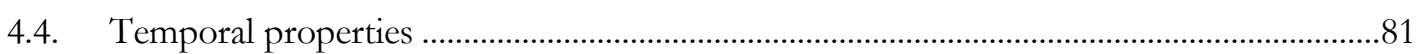

4.4.1. Temporal properties under full-field light steps .........................................................................82

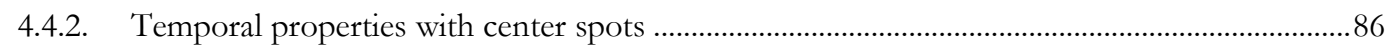

4.4.3. Are the temporal properties different between center and full-field stimulation? ....................8 88

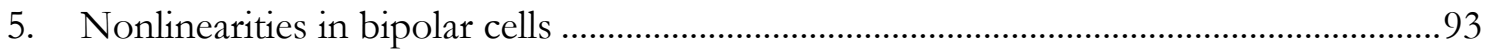

5.1. Nonlinearities in the output response function.................................................................93

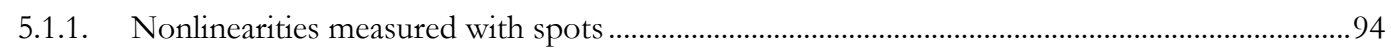

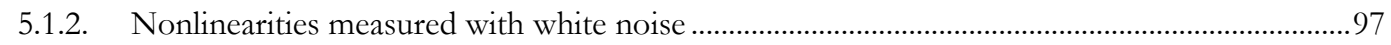

5.1.3. Are the nonlinearities with spots connected to the nonlinearities with white noise? ...............99

5.1.4. Are the output nonlinearities related to the general response properties?...............................100

5.2. Nonlinearities in the spatial integration ...................................................................... 102

5.2.1. Spatial integration in the receptive field center ...........................................................................103

5.2.2. Spatial integration in the receptive field surround...................................................................106

5.2.3. Is the spatial nonlinearity connected to the output nonlinearity?...........................................107

5.2.4. Is the spatial nonlinearity related to the general response properties? ....................................110

6. Linear-nonlinear model in bipolar cells ..........................................................................113

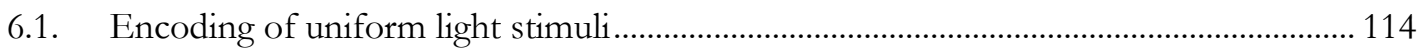

6.1.1. Prediction performance with uniform light stimuli .................................................................114

6.1.2. Is the prediction performance related to the observed nonlinearities? ....................................116

6.1.3. Is the prediction performance related to the general response characteristics?.......................117

6.2. Encoding of spatially structured light stimuli .............................................................. 119

6.2.1. Prediction performance with spatially structured light stimuli ...............................................120 
6.2.2. Is the prediction performance related to the observed nonlinearities?

6.2.3. Is the prediction performance related to the general response characteristics?

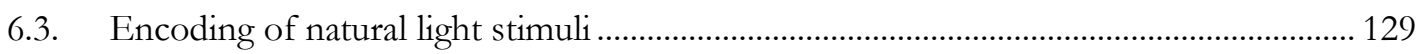

6.3.1. Do bipolar cells respond to natural light stimuli? ....................................................................129

6.3.2. Prediction performance with natural light stimuli........................................................................131

6.3.3. Is the prediction performance connected to the observed nonlinearities?..............................133

6.3.4. Adaptation under natural light stimulation..............................................................................136

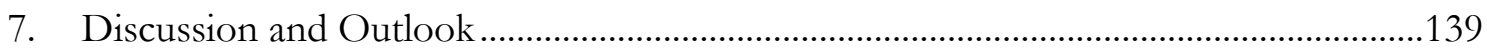

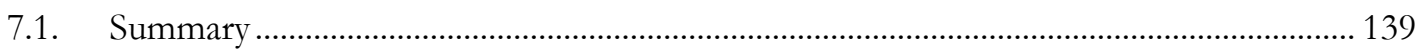

7.2. Role of nonlinearities for encoding visual signals in bipolar cells ............................... 141

7.3. Nonlinearities in bipolar cells: why they have not been observed before? ................... 146

7.4. Circuit mechanisms for nonlinearities in bipolar cell ................................................. 148

7.5. Consequences of nonlinearities in bipolar cells for models in retinal ganglion cells..... 155

7.6. Possible functions of bipolar cells' nonlinearities and linearities................................. 159

7.7. Challenges in quantifying bipolar cell response types ................................................ 164

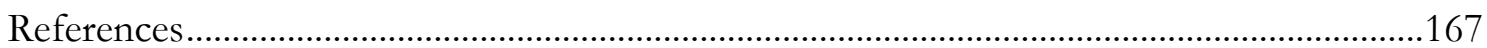





\section{List of figures}

Figure 1.1. Simple retina schema.

Figure 1.2. The two types of nonlinearities.

Figure 1.3. Linear-nonlinear model and its mapping to retinal anatomy.

Figure 2.1. The architecture of the eye and retina.............................................................................12

Figure 2.2. The five major cell classes and their subtypes in the retina............................................18

Figure 2.3. Spatial integration in retinal ganglion cells...................................................................25

Figure 2.4. Mechanism behind linear and nonlinear spatial integration in ganglion cells................28

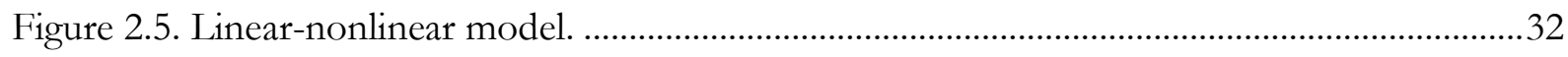

Figure 3.1. Combined recording technique (goal 4) ............................................................................. 38

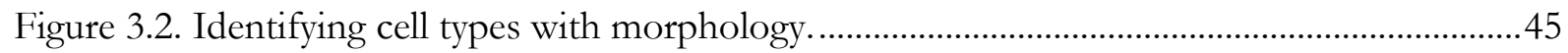

Figure 3.3. Retinal ganglion cell responses to current injection into a bipolar cell............................46

Figure 3.4. Light stimuli used for the general response characterization............................................49

Figure 3.5. Response polarity characterized with full-field light steps. ..............................................50

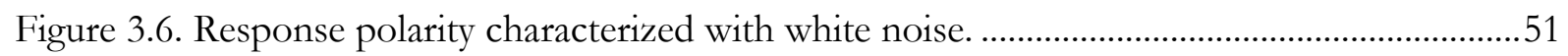

Figure 3.7. Receptive field center estimated with spots..................................................................52

Figure 3.8. Removing slow fluctuations from the membrane potential traces. ................................53

Figure 3.9. Receptive field center estimation with white noise. ...........................................................5

Figure 3.10. Temporal properties estimated with full-field light steps...............................................57

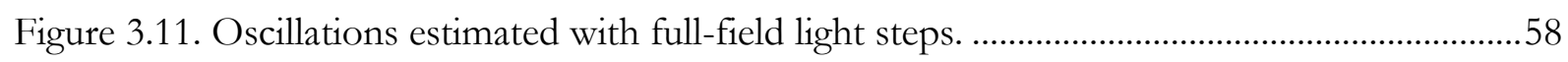

Figure 3.12. Estimating temporal properties with white noise analysis. ...........................................60

Figure 3.13. Estimation of the output nonlinearity with spots............................................................61

Figure 3.14. Estimation of the output nonlinearity with white noise................................................63

Figure 3.15. Stimulus designs to study spatial integration..................................................................64

Figure 3.16. Estimation of nonlinear spatial integration. .............................................................66

Figure 3.17. Implementation of the linear-nonlinear model for different light stimuli.................69

Figure 3.18. Overfitting in the linear-nonlinear model.......................................................................... 71

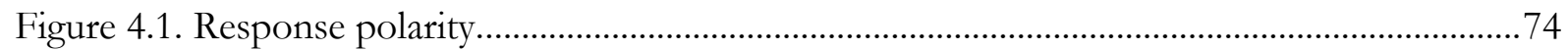

Figure 4.2. Investigating the response polarity with gray preframes. ................................................75

Figure 4.3. Center estimation with spots. ....................................................................................... 76

Figure 4.4 Receptive field estimation with spots without gray preframes. .........................................77

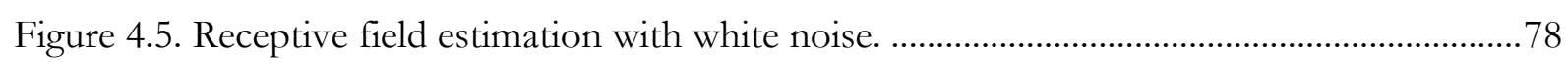

Figure 4.6. Comparison of receptive field sizes measured with spots vs. binary white noise..........80 
Figure 4.7. Receptive field surround estimation.

Figure 4.8. Response duration.

Figure 4.9. Oscillations in the responses of bipolar cells.

Figure 4.10. Response latency. .85

Figure 4.11. Temporal properties under a center spot stimulation. 86

Figure 4.12. Population distributions of the temporal properties to a center spot stimulation.......87

Figure 4.13. Comparison of the temporal properties under center and surround stimulation........89

Figure 4.14. Comparison of oscillatory responses under center and surround stimulation............90

Figure 4.15. Comparison of the response duration between white noise and spots.........................91

Figure 5.1. Output nonlinearity measured with spots......................................................................... 95

Figure 5.2. Output nonlinearity under center and surround stimulation..........................................96

Figure 5.3. Output nonlinearity with different spot designs............................................................97

Figure 5.4. Output nonlinearity with white noise ............................................................................99

Figure 5.5. Comparison of the nonlinear output transformation with spots and white noise......100

Figure 5.6. Output nonlinearity and the relation to general response properties............................102

Figure 5.7. Response traces for a linear and nonlinear spatial integration........................................104

Figure 5.8. Population distribution of the spatial nonlinearity index. ............................................105

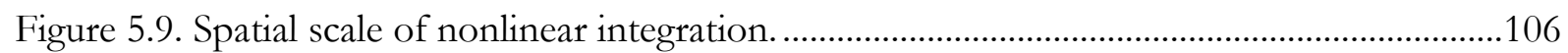

Figure 5.10. Nonlinear spatial integration under surround stimulation. ........................................107

Figure 5.11. Comparison of the spatial nonlinearity and the output nonlinearity..........................108

Figure 5.12. Predicting the nonlinear spatial integration with the black- white spot. ....................109

Figure 5.13. Predicting the nonlinear spatial integration with gray preframes...............................110

Figure 5.14. Relation of the spatial nonlinearity index to the general response properties...........111

Figure 6.1. Linear-nonlinear model (LN-model) under a full-field white noise stimulation.........115

Figure 6.2. Population distribution of the prediction performance with uniform stimuli...........116

Figure 6.3. Comparison of the prediction performance and the nonlinearities..............................117

Figure 6.4. Comparison of the prediction performance and the general response properties.....118

Figure 6.5. Linear-nonlinear model (LN-model) with binary white noise.......................................121

Figure 6.6. Population distribution of the prediction performance with spatial stimulus.............122

Figure 6.7 Nonlinear spatial integration leads to wrong prediction outputs. ...................................123

Figure 6.8. Comparison of the prediction performance to the spatial integration.........................124

Figure 6.9. The membrane potential range at around zero in the output function........................126

Figure 6.10. Performance outcome correlated with center and surround temporal properties. ..128

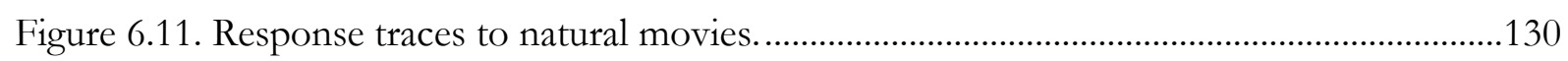

Figure 6.12. Prediction of natural movies' responses with the linear-nonlinear model.................132 
Figure 6.13. Natural movies prediction performance and nonlinear spatial integration. 133

Figure 6.14. Prediction performance of two natural movies for the same cell. ...............................135

Figure 6.15. Prediction performance with natural movies and binary white noise. ......................136

Figure 6.16. Linear-nonlinear model fitted with natural movies and white noise. ........................138

Figure 7.1 Assumed and proposed linear-nonlinear (LN) model. ...................................................143

Figure 7.2. Five possible mechanism for the nonlinearities observed in bipolar cells...................154

Figure 7.3. Implication of nonlinearities in bipolar cells for models in retinal ganglion cells.......158

Figure 7.4. Possible function of linear and nonlinear spatial integration........................................164 



\section{Introduction}

We start our journey with an overview from the general fascination of seeing to the specific questions I worked on during my doctorate. I begin my thesis with a brief glance into the retina, the door to our vision. Then, I outline the importance of studying bipolar cells and their nonlinearities for understanding the retinal circuitry. Further, I introduce the goals and questions I am addressing and I end this chapter with a general outline of my thesis.

\subsection{Retina, the door to vision!}

Vision is a precious sense with which we perceive the world. Every morning we wake up, open our eyes and see. We see the little fly washing its legs, the little honeybee that lost her way or the dance of a beautiful swallowtail butterfly. We just see! We see their movements, their little antennas, their wings, their patterning, their colors.

Seeing has long fascinated many researchers from different disciplines, psychologists, biologists, physicists to mention a few. They all try to understand the visual system from diverse perspectives. Yet still, vision remains mysterious and thus, so much more fascinating.

Seeing begins in the eye! Incoming photons are focused onto a thin neural tissue - the retina. The retina transforms photons into neural signals and transmits them via the optic nerve to various brain regions. The brain processes the visual world with the signals sent from the retina. Thus, the retinal signals are fundamental for seeing and every information lost at the level 
of the retina is lost for the brain too. Therefore, there is growing interest to understand what the "eye tells the brain"1.

To concretize, the output neurons of the retina are the retinal ganglion cells. Their axons assemble in the optic nerve and build the liaison to the brain. Research in the retina attempts to figure out what these retinal ganglion cells tell the brain. Do they report, similar to a camera, in a pixel-by-pixel fashion the light intensity? Or, do they extract and report specific "images", also referred to as "features", from the visual scene (e.g. contours, colors, moving object)? Decades of research portray that retinal ganglion cells indeed extract distinct visual features and transmit them in parallel streams to the brain (for a review see for example Gollisch and Meister (2010) or Roska and Meister (2014)). Currently, 30 different retinal ganglion cell types have been proposed in the mouse retina that disassemble the visual input into parallel "output channels" (Baden et al., 2016). What information each "output channel” encodes and how many exist, is subject of vivid debates. Yet, how do retinal ganglion cells become such diverse "output channels"? To understand the mechanisms that underlie the diverse response properties of retinal ganglion cells, we need to go back a step and learn about upstream neurons such as the bipolar cells.

\subsection{Why retinal bipolar cells?}

The bipolar cells represent the connecting element between the different neuronal types in the retina (see the simplified retina schema in Figure 1.1). In the outer retina, bipolar cells receive signals from the photoreceptors modified by the inhibitory feedback of horizontal cells. In the inner retina, bipolar cells transmit signals to ganglion cells modified by interactions with amacrine cells. Consequently, for transmitting signals from photoreceptors to ganglion cells, all information passes through the bipolar cells. This pivotal position makes them an interesting subject to study the mechanisms behind the response diversity observed in ganglion cells. Yet, this intermediate position makes it technically more challenging to record bipolar cells' neuronal responses. Therefore, so far, less research has been done on bipolar cells and our knowledge about "what bipolar cells tell retinal ganglion cells" is limited. Nevertheless, in various models, which try to explain the response properties of retinal ganglion cells, simplified assumptions are made about bipolar cells' response characteristics, for example, that they respond to light linearly.

\footnotetext{
${ }^{1}$ The expression refers to an article published by Lettvin et al. (1959) under the title "What the Frog's Eye Tells the Frog's Brain.
} 


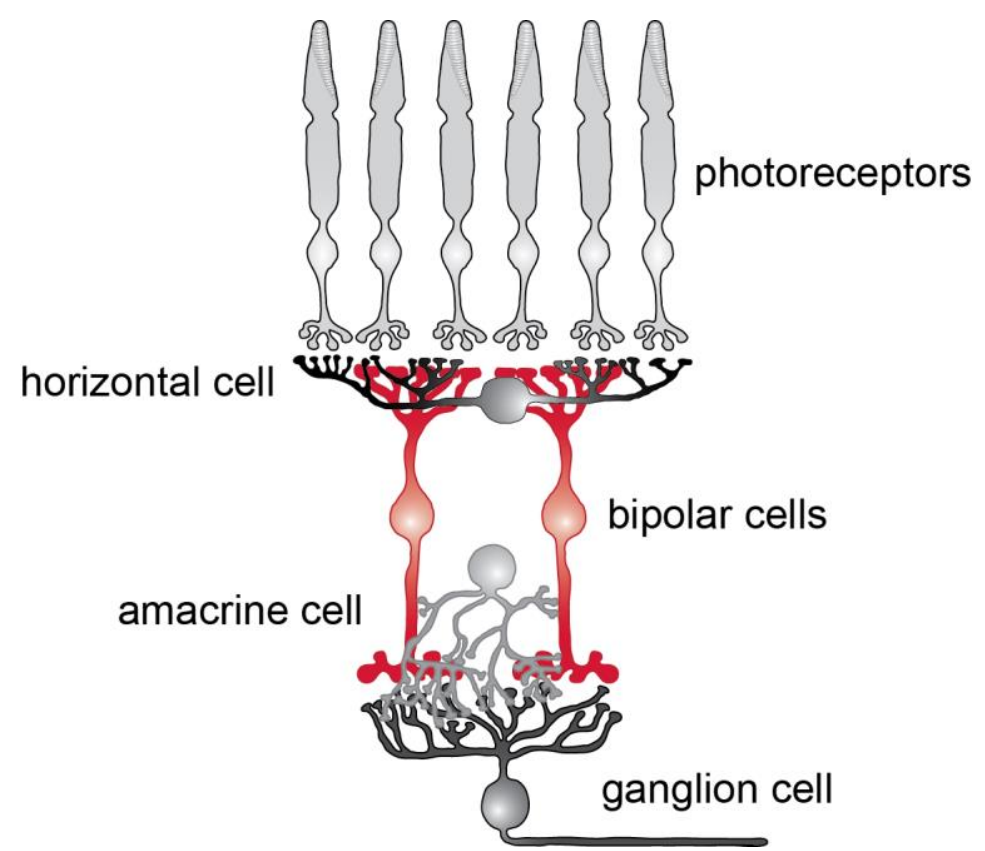

Figure 1.1. Simple retina schema. The photoreceptors absorb the light and transmit the neuronal signals modified by horizontal cells to the bipolar cells (outer retina). The bipolar cells forward the signals modified by amacrine cells to the ganglion cells (inner retina). The schematic retina in the current and following figures was inspired by Wassle (2004) and Swaroop et al. (2010).

\subsection{Why nonlinearities?}

Studying whether neurons respond to light in a linear or nonlinear way is investigated at two stages in a neuron: at its output and at its inputs. Traditionally, the nonlinearity at the output is examined. A common way to measure the nonlinear output function in visual neurons is by setting a reference light level (e.g. gray) from which the contrast is increased (e.g. to white) or decreased (e.g. to black) by equal amounts (Fig. 1.2A). The response of the neuron is measured (e.g. membrane potential) to the reference level and compared to the increases and decreases in contrast. If a neuron increases its membrane potential to one contrast (e.g. $+3 \mathrm{mV}$ to white) and decreases the voltage to the opposite contrast by the same amplitude (e.g. - $3 \mathrm{mV}$ to black), the cell is termed linear (Fig.1.2A). If however, the neuron increases and decreases the membrane potential with different amounts $(0 \mathrm{mV}$ to black and $+3 \mathrm{mV}$ to white) the cell is termed nonlinear. Often, bipolar cells are approximated by a linear response to light. The view is reinforced by the notion that bipolar cells, similar to photoreceptors and horizontal cells, are non-spiking neurons that respond to light with graded potentials. Retinal ganglion cells, and some amacrine cells, on the other hand, are spiking neurons. Here, the response to light is often approximated nonlinearly. For example, weak light inputs give rise to no response and only if the input stimulus 
passes a threshold, the neuron spikes. Further, retinal ganglion cells might show response saturation, for example, at a certain point, a further increase in the input magnitude does not increase the spiking rate any further. The described linear and nonlinear responses to light are summarized under the terms stimulus-response transformation, output function or output nonlinearity ${ }^{2}$. All terms are used in this thesis as synonyms.

Another way to measure whether neurons are linear or nonlinear is by studying the spatial integration. A neuron typically receives inputs from multiple upstream neurons. How a neuron combines these inputs into an output is described by its spatial integration property. Thus, here, space refers to light input signals at different spatial locations that activate different presynaptic neurons (Fig.1.2B). Measuring the spatial integration is not as straightforward as for the stimulusresponse function. By a set of groundbreaking experiments, Enroth-Cugell and Robson (1966) presented a dark half and a light half inside the receptive field of retinal ganglion cells (see Fig.1.2B for a simplified version of the stimulus). The idea behind the experiment was to assess spatial integration by presenting both positive (e.g. white contrast +1 ) and negative (e.g. black contrast -1) activation inside the receptive field and study whether the activations with opposite signs can cancel out the response (with the logic of $-1+1=0)$ or not $(-1+1>0)$. They found that some retinal ganglion cells indeed remained silent when presenting such a stimulus and concluded that for those cells the summation of the presynaptic inputs was approximately done linearly (-1+1=0, Fig.1.2B.) Curiously, they also found retinal ganglion cells that clearly responded to such light combinations, thus, here the summation over the presynaptic inputs was nonlinear $(-1+1>0$, Fig.1.2B). The described linear and nonlinear responses are studied under the terms spatial integration, input nonlinearity or spatial nonlinearity ${ }^{3}$. All terms are used as synonyms.

The stimulus-response transformation and spatial integration are studied by separate stimulus designs. Thus, for each neuron two main types of nonlinearities are generally distinguished in the retina: the output nonlinearity and spatial nonlinearity (Fig.1.2).

\footnotetext{
${ }^{2}$ The term output nonlinearity to describe a linear transformation might be counterintuitive. It has its origin from spiking neurons that have internal nonlinear processes like a spike threshold or a saturation. In this thesis, we will always specify whether the measured output nonlinearity was linear or nonlinear.

${ }^{3}$ As for the output nonlinearity, the term spatial or input nonlinearity might be counterintuitive to describe a linear integration. In this thesis, we will always specify whether the spatial integration was linear or nonlinear.
} 
A

Output nonlinearity

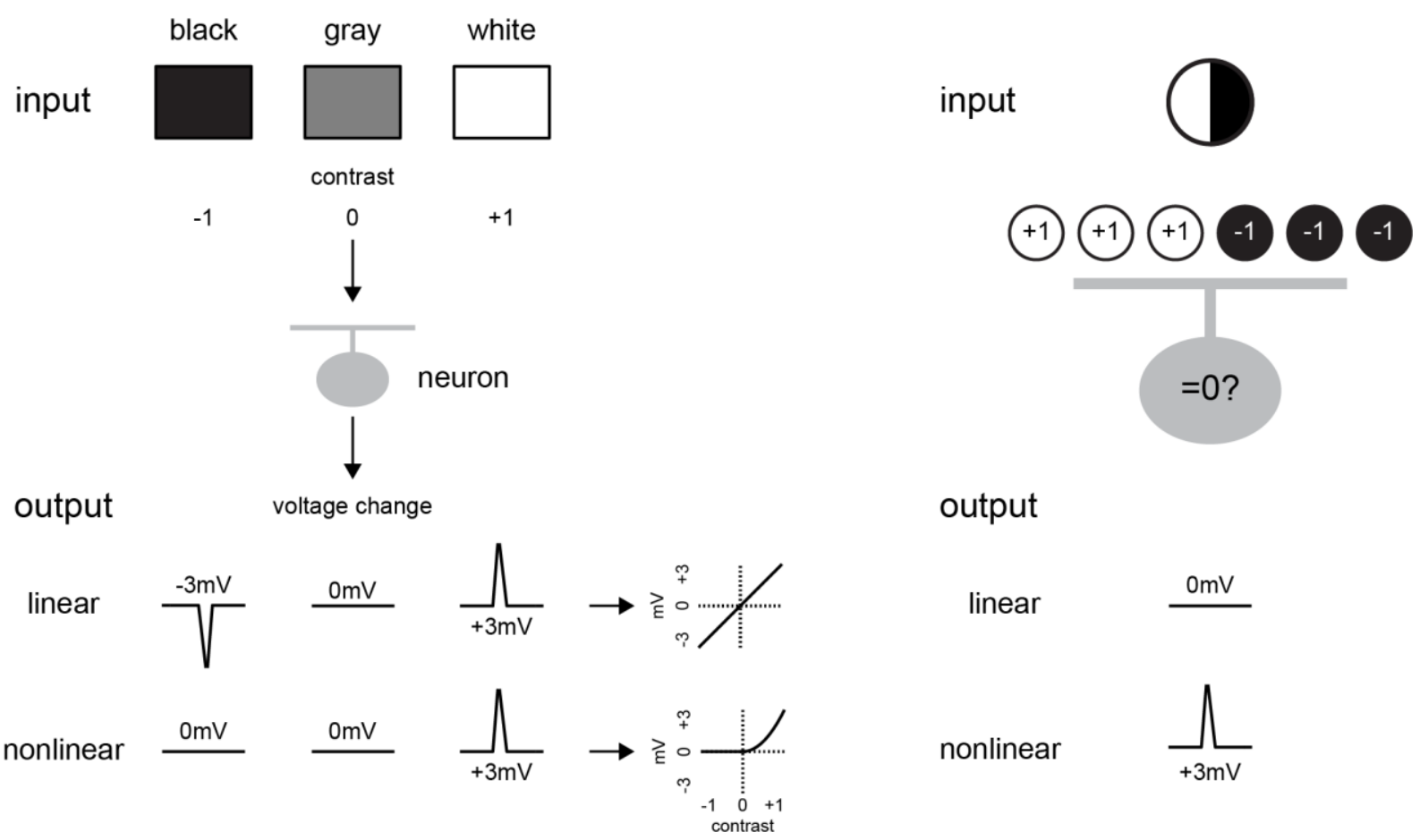

Figure 1.2. The two types of nonlinearities. A. Output nonlinearity, also termed stimulus-response transformation, here the light signal (input) is related to the neuronal response (output). A reference light level is set (e.g. gray) from which the contrast is increased (e.g. white, in Weber contrast +1 ) and decreased (e.g. black, in Weber contrast -1). The neuron's output is measured in millivolts. A linear cell responds with equal amount of negative and positive deflection from the voltage at the reference light level. A nonlinear cell responds with different amounts. B. Spatial nonlinearity, also termed spatial integration, here the input neurons are stimulated with dark (-1, in Weber contrast) and bright ( +1 , in Weber contrast) contrast. If the activation with opposite sign cancels out the response $(-1+1=0)$, the cell is termed linear, if however the cell responds $(-1+1 \neq 0)$ it is termed nonlinear.

It has been proposed that at the heart of the feature extraction, and thus response diversity in retinal ganglion cells, lie nonlinear signal transformations from bipolar cells to ganglion cells (Gollisch, 2013; Gollisch and Meister, 2010; Roska and Meister, 2014). For example, nonlinear spatial integration in retinal ganglion cells cannot be explained by a linear signal transmission. Further, linear signal transmission to ganglion cells cannot explain the response to small objects that move differently from the background detected by object-motionsensitive ganglion cells originally termed "bug perceiver" (Baccus et al., 2008; Lettvin et al., 1959; Ölveczky et al., 2003; Zhang et al., 2012) or the sensitivity of some ganglion cells to approaching objects, also termed "approach-sensitive" or "looming detectors" (Munch et al., 2009). 
Yet, studies measuring the stimulus-response relationship in bipolar cells' somas show controversial results. Certain studies show a linear stimulus-response relationship (Baccus and Meister, 2002; Dacey et al., 2000; Fahey and Burkhardt, 2003; Rieke, 2001; Sakai and Naka, 1987a; Toyoda, 1974) and other studies reported nonlinear bipolar cell responses (Burkhardt and Fahey, 1998; Euler and Masland, 2000; Fahey and Burkhardt, 2003). It has been speculated that the inconsistency between the different studies is due to different stimulus dynamics and bipolar cell types (Burkhardt and Fahey, 1998; Schwartz and Rieke, 2011). Yet, whether different stimuli produce different nonlinear properties, as well as whether different bipolar cell types (e.g. sustained vs. transient) show different nonlinearities, is not understood. Moreover, how the particular form of the nonlinear stimulus-response relationship in bipolar cells looks like is unclear. Traditionally in computational models, they are approximated by a threshold-linear transformation (Gollisch, 2013; Gollisch and Meister, 2010), yet recently also threshold-quadratic transformations were proposed (Bolinger and Gollisch, 2012).

In addition, how bipolar cells themselves integrate their inputs from presynaptic photoreceptors and horizontal cells is not known. Yet, the spatial integration in bipolar cells is broadly assumed to occur linearly. However, evidence for nonlinear neurotransmitter release in photoreceptors, is challenging the view of a linear integration in bipolar cells. For example nonlinear signals in rods (Dunn and Rieke, 2008; Field and Rieke, 2002; van Rossum and Smith, 1998) and in cones (Baden et al., 2013c; Dunn et al., 2007) have been reported.

\subsection{Nonlinearities: the burden for encoding!}

Neural encoding is the study of the stimulus to response "dictionary" and an important concept when trying to understand what the "eye tells the brain". The idea is to characterize how neurons respond to various stimuli, and then build models that aim to predict responses to new stimuli (Dayan and Abbott, 2001). To deduce what neurons encode ("tell"), computational models are essential. One of the most important models to understand neural encoding in the retina is the linear-nonlinear model (LN-model) (Chichilnisky, 2001; Gollisch, 2013).

The linear-nonlinear model has mainly been applied to study responses in retinal ganglion cells. The model and its mapping onto retinal anatomy is illustrated in Figure 1.3 for a retina ganglion cell. The idea is that the light stimulus is first linearly passed through the input neurons, then summed by the retinal ganglion cell, and transformed through a cell's internal nonlinear process (spike threshold and saturation) to generate a response. While this simple model manages to predict responses to simple uniform contrast stimuli quite accurately (Chichilnisky, 2001; 
Zaghloul et al., 2007), it fails for light stimuli with artificial and natural spatial structure (Freeman et al., 2015; Heitman et al., 2016; Liu et al., 2017). One reason for the failure is seen in nonlinear signal transmission from bipolar cells to ganglion cells (Gollisch, 2013; Schwartz and Rieke, 2011). The visual signals are not simply linearly transmitted in the retina, but nonlinearly transformed at the bipolar cell level, before the summation in the retinal ganglion cells. Further, the linear-nonlinear model assumes a complete linear transmission from photoreceptors to bipolar cells. If linear signal transmission would occur until bipolar cells, the simple linearnonlinear model should be able to describe bipolar cells' responses to both artificial and natural stimuli accurately. Yet, investigating the linear-nonlinear model with bipolar cells has been largely neglected in the retina field.

Anatomy

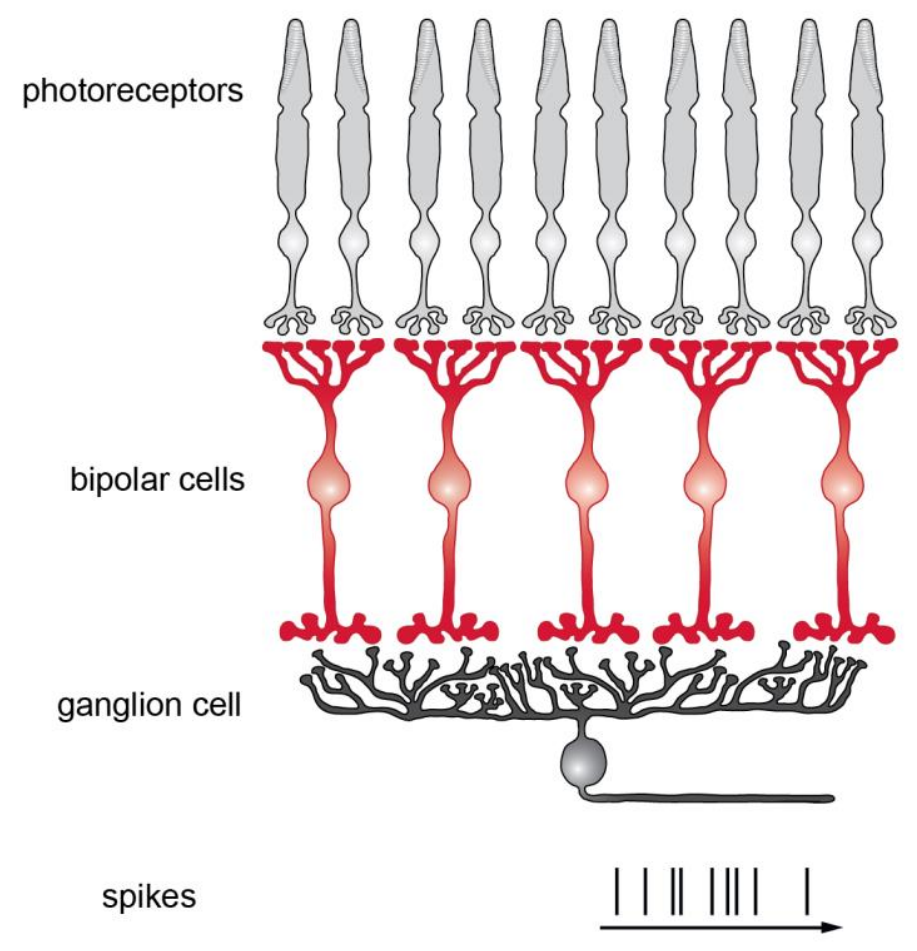

Linear-nonlinear-model

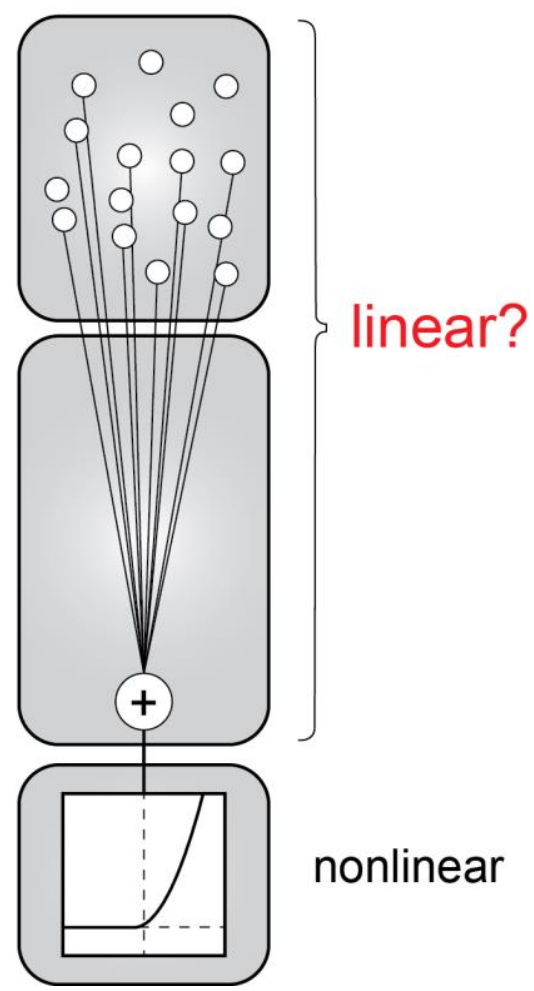

Figure 1.3. Linear-nonlinear model and its mapping to retinal anatomy. In the linear-nonlinear model (LN-model) a complete linear signal transmission is assumed from the photoreceptors to the ganglion cells. A retinal ganglion cell pools the linear signals and nonlinearly transforms them through a cell internal nonlinear process (spike threshold and saturation) to generate spikes. Yet, is the signal transmission from photoreceptors to ganglion cells through the bipolar cells linear? The figure was inspired by Freeman et al. (2015). 


\subsection{Goals of this thesis}

Four goals were pursued in this dissertation. The first three goals focus on bipolar cells' response properties. The fourth goal focus on establishing a method for simultaneous recordings in bipolar and retinal ganglion cells by combining intra- and extracellular recording techniques.

\section{Goal 1: Establishing a general characterization of bipolar cells}

In the first goal we aimed to describe bipolar cells based on classical response properties (e.g. sustained, transient, ON, OFF, receptive field size). To do so, we studied the bipolar cells' responses to diverse light stimuli. Moreover, we investigated the bipolar cells' center-surround structure and asked whether their responses change under center and surround stimulation.

\section{Goal 2: Assessing nonlinearities in bipolar cells}

In the second goal of this thesis, the two types of nonlinearities were studied in bipolar cells. First, the stimulus-response transformation (output nonlinearity) was investigated. To do so, two sets of stimuli were used; a simple flashing spot stimulus and a flickering contrast sequence. In a next step, the second type of nonlinearity, the spatial integration properties of bipolar cells (spatial nonlinearity) was investigated. Here, we performed experiments that have originally been conducted in retinal ganglion cells (Enroth-Cugell and Robson, 1966), in bipolar cells. Thus, dark and light patches were shown inside the bipolar cell's receptive field and we investigated whether bipolar cells sum signals from their input neurons linearly or nonlinearly. Further, we investigated the role of the surround and whether different bipolar cell types (e.g. sustained vs. transient) exhibit different nonlinearities.

\section{Goal 3: Predicting bipolar cells' responses with the linear-nonlinear model}

For the third goal, the bipolar cell responses were studied with the linear-nonlinear model. Here, we were curious whether bipolar cells' responses could be predicted with the model, which would point to a linear signal transmission from photoreceptors to bipolar cells. We investigated the model accuracy with three different types of stimuli: two artificial light stimuli and one natural light stimulus. We asked whether the model could predict responses to artificial stimuli with and without spatial structure. Additionally, we were curious how bipolar cells respond to natural stimuli and whether their responses can be predicted with the linearnonlinear model. 


\section{Goal 4: Simultaneous recordings from bipolar and ganglion cells}

Here, we established a method for simultaneous recordings in bipolar and retinal ganglion cells by combining intra- and extracellular recording techniques. The technical approach is similar to Asari and Meister (2012), yet we use a perforated multielectrode array. We simultaneously recorded single bipolar cells with sharp microelectrodes and large numbers of nearby ganglion cells with a multielectrode array. We used the simultaneous recording technique to verify whether mathematically identified subunits from recorded ganglion cells correspond to actual bipolar cell receptive fields (Liu et al., 2017).

\subsection{Outline of this thesis}

After the introduction, we continue our thesis with an overview of the literature of the retina (chapter 2). First, we present a detailed picture of the five major cell types (subchapter 2.1) looking at their morphology, their neurotransmitters as well as receptor types. Then, we continue with a historical overview of the physiological measurements of light responses (subchapter 2.2). Further, we inspect the literature about the nonlinearities in the retina (subchapter 2.3) and we end the chapter with an introduction into the computational models in the retina (subchapter 2.4).

In the next chapter (chapter 3), we describe the experimental and analytical methods used in this thesis. Here, we elaborate the combined recording technique of goal 4 that allows simultaneous recordings from bipolar and ganglion cells.

The results are presented in three chapters. In the first chapter (chapter 4), the results for the general response characterization are shown (goal 1). In the next chapter (chapter 5), we study the two types of nonlinearities in bipolar cells (goal 2). In chapter 6, we present the analysis with the linear-nonlinear model for artificial and natural stimuli (goal 3).

Finally, we summarize and discuss the results in chapter 7 . Thereby, we investigate the implication of the findings for encoding visual signals in bipolar cells as well as mathematical models in the retina. Furthermore, we introduce new ideas that could enrich the current findings. 



\section{Background: Research in} the vertebrate retina

More than a century of morphological research, more than half a century of physiological research and decades of computational modeling have built the understanding of retinal signal processing (Baccus, 2007). In this chapter, we review the three major lines of research in the vertebrate retina and show how their combination leads to our understanding of retinal function. First, we start by reviewing the anatomical structure of the retina and by presenting a detailed picture of the five major cell types (subchapter 2.1). More precisely, we look at the synaptic connections, the main neurotransmitter and receptor types. We then continue with a detailed literature overview of the physiological measurements of light responses in the retina. Here, we investigate the general response characterizations (subchapter 2.2 e.g. ON, OFF, transient, sustained) and the nonlinearities (subchapter 2.3). Finally, we examine the computational models in the retina (subchapter 2.4). Thereby, we focus on the linear-nonlinear model. At each stage, we introduce the previously discussed goals in the context of the literature.

\subsection{The architecture of the retina}

The eyes and retinas among vertebrates show a common architecture (Rodieck, 1998). Light is reflected by the cornea, passed through the pupil to the lens and focused at the back of the eye onto the retina (Fig.2.1A). The retina is a thin neural tissue of $\sim 200-300 \mu \mathrm{m}$ thickness and is part of the brain itself (Sterling and Demb, 2004). 
The vertebrate retinas are composed of five major classes of neurons arranged in three nuclear and two synaptic layers (Fig.2.1B). The light travels through the five layers before it reaches the outer segment of the light-sensitive photoreceptor cells, which nucleus are situated in the outer nuclear layer (ONL). Here, the light signals are transduced to neural signals. In the first synaptic layer, the outer plexiform layer (OPL), bipolar cells receive the signals from the photoreceptors, modified by the inhibitory feedback of horizontal cells. In the second synaptic layer, the inner plexiform layer (IPL), bipolar cells transmit signals to ganglion cells, modified by interactions with amacrine cells. The cell bodies of the bipolar, horizontal and amacrine cell lie in the inner nuclear layer (INL), the cell bodies of the ganglion cells in the ganglion cell layer (GCL). The retinal ganglion cells, the output neurons of the retina, are situated towards the inside of the eye, and their axons form the optic nerve.

A

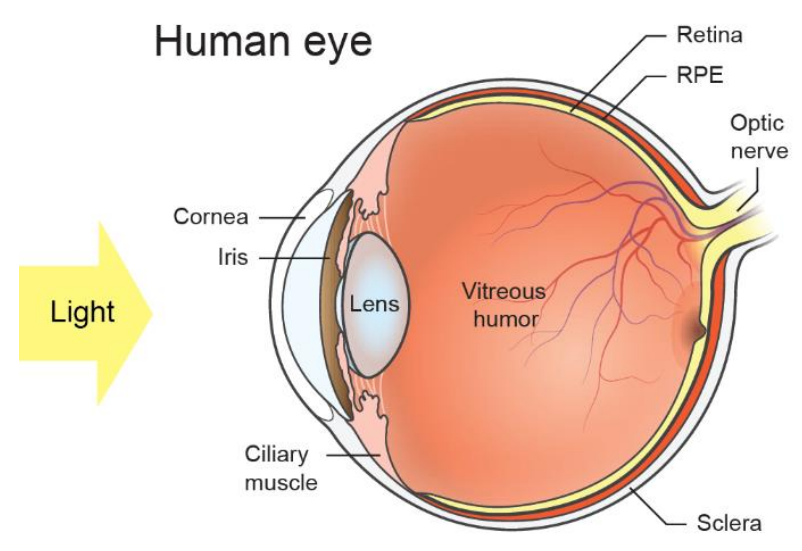

B

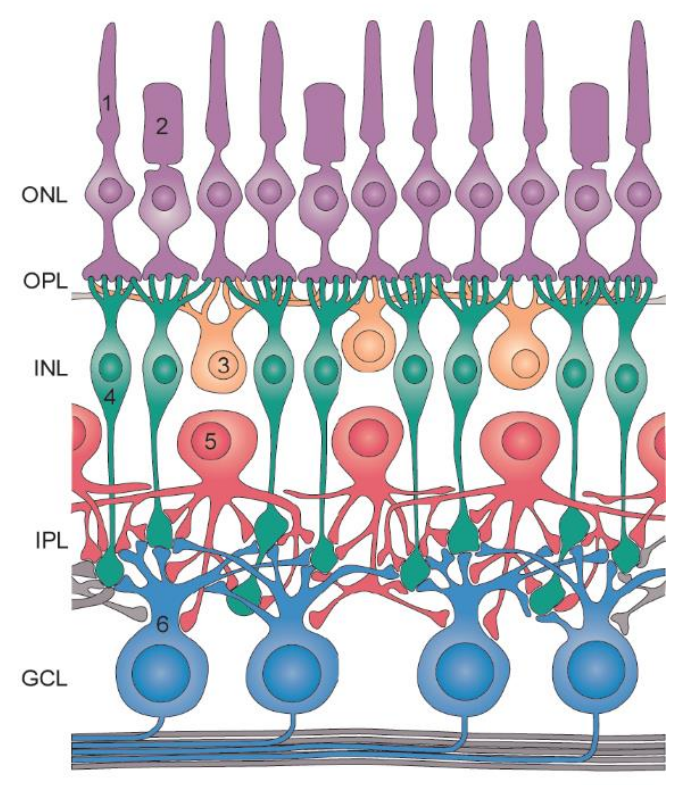

Light

Figure 2.1. The architecture of the eye and retina. A. The cornea is transparent and covers the front of the eye. The light passes through the pupil, the hole in the iris, and is focused by the cornea and lens onto the retina. There, the light travels first through the retina before it reaches the light sensitive photoreceptor cells that are embedded into pigment epithelium (RPE). B. Schematic architecture of the retina. The outer nuclear layer (ONL) contains the cell bodies from rod (1) and cone (2) photoreceptors. The outer plexiform layer (OPL) contains the photoreceptor-bipolar-horizontal cell synapses. The inner nuclear layer (INL) contains the cell bodies from horizontal (3), bipolar (4) and amacrine (5) cells. The inner plexiform layer (IPL) contains the bipolar-amacrine-ganglion cell synapses. The ganglion cell layer (GCL), contains the cell bodies from the ganglion cells. Images adapted from Veleri et al. (2015) and Wassle (2004). 


\section{The retinal cell types}

Each of these five major neuron classes is further subdivided into several or many individual types (Masland, 2004). We are going to have a closer look at each major cell class and highlight some of the important subtypes (see Fig.2.2). Further, we look at their morphological structure, neurotransmitter and receptor types.

Photoreceptors. The outer segments of photoreceptors contain light-catching proteins (commonly referred to as photopigments) that undergo a structural change when hit by light. The process is termed photoisomerization and initiates a signaling cascade (phototransduction), which closes ion channels and leads to a hyperpolarization of the photoreceptor cell (Rodieck, 1998). Thus, photoreceptors depolarize, i.e. release neurotransmitter, in darkness and hyperpolarize, i.e. reduce neurotransmitter release, with light. Photoreceptors release the excitatory neurotransmitter glutamate.

There are two main types of photoreceptor cells: rods and cones. They can be distinguished by morphology, rods have long outer segments, and cones have a conoid geometric shape (see Fig.2.2). Max Schultze (1866) proposed a duplex theory of vision, where rods are active during low-light level (scotopic vision), whereas cones are active during daylight (photopic vision) and different studies confirmed the idea (e.g. Aguilar \& Stiles (1954), Fain \& Dowling (1973), for a review see also Ingram et al. (2016)). However, some recent studies in mammalian retina report rod activity during high-light levels (Tikidji-Hamburyan et al., 2017). At intermediate light levels (mesopic vision), both rods and cones are active.

Each photoreceptor type has a different photopigment that defines the wavelength sensitivity. In the mammalian retina, only one type of rod has been described, in the salamander retina two types of rods are reported. The "red" rods $(\sim 98 \%)$ are sensitive to middle wavelength ( $\sim$ green light), the "green" rod $(2 \%)$ to short wavelength ( blue light) (Sherry et al., 1998). The naming of "red" and "green" is confusing and has been applied unrelated to the spectral sensitivity, alternative names are s- and m-rods (Sherry et al., 1998). For the cones, 2-3 types have been reported in the mammalian retina. Humans for example are trichomats with three cone types, the short- (blue), the medium- (green) and the long-wavelength sensitive (red) cone. The mouse retina has two types of cones, one UV-and one medium-wavelength sensitive cone. For salamander retina four cone types have been identified, one large and one double-cone (two outer segments) sensitive to long-wavelength $(\sim 84 \%)$, one small cone type sensitive to shortwavelength $(\sim 8 \%)$ and one UV-cone type (7\%) (Sherry et al., 1998). In most vertebrate retinas, rods outnumber cones, for example in the mouse retina $\sim 3 \%$ are cones and $\sim 97 \%$ are rods (Jeon et al., 1998), in the salamander retina 38\% are cones and 62\% are rods (Wang et al., 2016). In some vertebrate retinas some type of cones appear more numerous in one region of the retina 
(Applebury et al., 2000), however no such arrangement was observed in the salamander retina (Sherry et al., 1998).

Photoreceptors transmit their signals through glutamate release to horizontal and bipolar cells and we will look at these two downstream neurons in the next sections.

Horizontal cells. Both rods and cones synapse onto horizontal cells. Horizontal cells have ionotropic AMPA and kainate-type glutamate receptors and therefore hyperpolarize to light. They are characterized by dendritic branches that laterally spread out in the inner plexiform layer with cell bodies in the inner nuclear layer.

Horizontal cells feed back onto photoreceptors and thus influence the photoreceptor glutamate release. How the horizontal-to-photoreceptor feedback is employed is still controversial. Currently, three potential mechanism are debated (Chapot et al., 2017; Wassle, 2004). The classical mechanism assumes that horizontal cells release the inhibitory neurotransmitter GABA, which affects the photoreceptor transmitter release at the synaptic terminals (Wassle, 2004). This idea has been challenged due to the lack of ionotropic GABA receptors in mouse cones (Kemmler et al., 2014). Two alternative mechanisms have been proposed (Chapot et al., 2017; Wassle, 2004). One acts through ephaptic coupling, here cations flow through hemichannels (e.g. connexins) into the synaptic cleft (fast mechanism) (Chapot et al., 2017; Wassle, 2004). The other mechanism is a proton-mediated feedback, where voltagedependent ion transport of horizontal cells change the $\mathrm{pH}$ concentration in the synaptic gap (slow mechanism) (Chapot et al., 2017; Wassle, 2004). Both mechanism act on voltage-gated calcium channels expressed at the photoreceptor synaptic terminals and thereby change the glutamate release.

It still remains controversial whether horizontal cells directly influence bipolar cells or whether they only indirectly change the bipolar cell signal over the described feedback mechanism onto photoreceptors (Chapot et al., 2017; Masland, 2012a). There is however, evidence of GABA receptors on bipolar cell dendrites and thus horizontal cells might directly influence bipolar cells in a feedforward pathway (Hoon et al., 2015; Puller et al., 2014; Yang and Wu, 1991).

The exact function of horizontal cells in the retina circuitry is still only partially understood (Chapot et al., 2017). For example, horizontal cells seem to integrate signals over a broad region through their spread out dendrites and gap junctions. Dependent on the animal species around 1-3 types of horizontal cells are described (see Fig.2.2), for example 1 type in the mouse retina (Peichl and Gonzalez-Soriano, 1994), 2 types in primates (Dacey et al., 1996) and 3 
types in the salamander retina (Zhang et al., 2006). Around 5\% of the total amount of cells in the retina are horizontal cells (Masland, 2001).

Bipolar cells. The term "bipolar cell" has been attributed to Tartuferi, an apprentice of Golgi, and describes retinal cells with two prolongations, one going up and one going down (Euler et al., 2014). This idea still holds nowadays, bipolar cells are the retinal cells that make synaptic contacts both in the outer and inner plexiform layer.

In the outer plexiform layer, bipolar cells receive glutamatergic input from photoreceptors. Bipolar cells do not uniformly respond to the glutamate release, instead, they start to shape the signal differently based on their own glutamate receptor types. Two broad classes of bipolar cells can be differentiated in the vertebrate retina. One bipolar cell class expresses ionotropic glutamate receptors (iGluRs) (Brandstatter et al., 1997; Morigiwa and Vardi, 1999; Peng et al., 1995; Qin and Pourcho, 1996). Here, glutamate released by photoreceptors in darkness opens the cation channels at the bipolar cell dendrites. The receptor is thus signconserving and the bipolar cell depolarizes in the dark like the photoreceptor. Two types of ionotropic glutamate receptors have been reported in bipolar cells: AMPA and kainate receptors (Brandstatter et al., 1997; DeVries, 2000; DeVries and Schwartz, 1999; Morigiwa and Vardi, 1999; Peng et al., 1995; Qin and Pourcho, 1996). The other class of bipolar cells expresses metabotropic glutamate receptors (mGluR6) (Nomura et al., 1994). Here, glutamate initializes a cascade that through a second-messenger closes cation channels and hyperpolarizes the cell. The receptor is therefore sign-inverting and the bipolar cell hyperpolarizes in the dark.

The axons of bipolar cells stratify into the inner plexiform layer. The inner plexiform layer is subdivided into five sublaminas. In vertebrates, it has been observed that the axons of the two broad classes of bipolar cells (ionotropic vs. metabotropic receptors) stratify at different depth in the inner plexiform layer (Hare et al., 1986). The bipolar cells with ionotropic receptors stratify into the layers near the amacrine cell bodies and the bipolar cells with metabotropic receptors stratify into the layers close to ganglion cell bodies. These findings started the idea that bipolar cells can be subdivided into different types based on their anatomy which includes the stratification level, the dendritic branching pattern, the number and type of photoreceptor contacts (Euler et al., 2014; Ghosh et al., 2004; Wassle and Boycott, 1991). Further, the classification of bipolar cells is complemented by immunomarker labelling (Wassle et al., 2009). In the mouse retina, $\sim 13-15$ morphological types of bipolar cells are reported (see also Fig.2.2) (Euler et al., 2014; Ghosh et al., 2004; Helmstaedter et al., 2013; Tsukamoto and Omi, 2017; Wassle et al., 2009). The most numerous bipolar cell type is the rod bipolar cell which contacts only rods (Euler et al., 2014). All other bipolar cell types contact cone photoreceptors, some of 
them -as more recently described- make additional contacts with rod photoreceptors (Haverkamp et al., 2008; Mataruga et al., 2007). Except for two cone bipolar cell types, that exclusively contact s- or m-cones, the contacts are mixed between $\mathrm{m}$ - and s-cones (Breuninger et al., 2011; Haverkamp et al., 2005). The cone bipolar cells make contacts to 5-12 cones (Wassle et al., 2009), rod bipolar cells contact between 20-80 rod spherules (Wassle, 2004).

In the salamander retina, a similar number of $\sim 12-20$ bipolar cell types has been reported (Pang et al., 2004; Wang et al., 2016; Wu et al., 2000). Unlike in the mouse retina, but similar to fish or human retinas (Kolb et al., 1992), some bipolar cells stratify in multiple sublaminas in the inner plexiform layer (bi- or tristratification) or show axonal branching, which makes the bipolar cell types more diverse (Pang et al., 2004; Wu et al., 2000). Further, the salamander retina also has rod or cone dominated bipolar cells and multiple "mixed" bipolar cells contacting both rods and cones (Wu et al., 2000). Whether there are exclusive contacts to one specific type of cone or rod (specific photopigment), is not clear.

As mentioned in the previous section, bipolar cells might receive GABAergic input from horizontal cells in the outer plexiform layer (Hoon et al., 2015; Puller et al., 2014; Yang and Wu, 1991). However, this topic is controversial and it is not yet known whether different bipolar cell types would express different GABA receptors (see Hoon et al. (2015) who studied four different bipolar cell types and found that they all have the same $\mathrm{GABA}_{\mathrm{A} \text { alpha1 }}$ receptor).

The diversity of bipolar cells' morphology (see Fig.2.2), receptors and the influence of the horizontal cells is the first candidate for the diversification of the visual signal in the retina. Bipolar cells release glutamate onto amacrine and ganglion cells and we further introduce these two cell classes.

Amacrine cells. Amacrine cells are the most diverse and least understood retinal cell class (Masland, 2012b). Around $\sim 42$ different types of amacrine cells are estimated (see also Fig.2.2), but only very few have been studied (Euler et al., 2014). Two main types of amacrine cells are broadly distinguished: "small-field" and "wide-field", based on the size of their dendritic trees (Franke and Baden, 2017). Amacrine cells collect the signal across the different depth of the inner plexiform layers both vertically or laterally.

Amacrine cells are directly activated through glutamate release from bipolar cells and express ionotropic glutamate receptors such as AMPA, kainate and NMDA (Dumitrescu et al., 2006; Sterling and Demb, 2004). Further, amacrine cells release two main types of inhibitory neurotransmitters: GABA and glycine. In the mammalian retina, small-field amacrine cells are mainly releasing glycine (Menger et al., 1998) and wide-field amacrine cells release GABA (Pourcho and Goebel, 1983). This is in contrast to the salamander retina, where small-field 
amacrine cells mainly release GABA and wide-field amacrine cells release glycine (Yang et al., 1991). In addition, some amacrine cells also release modulatory neurotransmitter like e.g. dopamine (Contini and Raviola, 2003), serotonin (Li et al., 1990), acetylcholine (Masland and Mills, 1979) and glutamate (Lee et al., 2014). These neurotransmitters might be co-released with other neurotransmitters (e.g. GABA) (Euler et al., 2014; Masland, 2012b; Sterling and Demb, 2004).

In the inner plexiform layer amacrine cells release different types of neurotransmitters onto bipolar cells and ganglion cells and they provide feedback to amacrine cells themselves. Thus, they use their dendrite as a site for both receiving inputs and releasing outputs (Euler and Denk, 2001). Further, they are coupled with bipolar, ganglion and other amacrine cells through gap junctions. Bipolar, ganglion and amacrine cells express a diversity of target receptors for amacrine cells. The diversity of neurotransmitter and receptor types at the inner plexiform layer is another candidate for the diversification of the visual signal in the retina. However, which exact type of receptor each bipolar, ganglion or amacrine cell type expresses is not yet known. To just name a few target receptors of amacrine cells, both ionotropic $\mathrm{GABA}_{\mathrm{A}}$ and $\mathrm{GABA}_{\mathrm{C}}$ receptors (Shields et al., 2000), metabotropic $\mathrm{GABA}_{\mathrm{B}}$ receptors, ionotropic and metabotropic glycinergic receptors (Hou et al., 2008) as well as nicotinic receptors (Zucker and Yazulla, 1982) have been localized in the inner plexiform layer (see Zhang and McCall (2012) for a review of receptor types in the inner plexiform layer). Further, there is evidence for cell type specific receptor expression, for example $G A B A_{C}$ is primary located in bipolar cells whereas $G_{A B A}$ seems to be present in bipolar as well as ganglion and amacrine cells (Sterling and Demb, 2004; Zhang and McCall, 2012). Glycinergic receptors have not been observed in metabotropic cone bipolar cells, but in ionotropic and rod bipolar cells as well as ganglion and amacrine cells (Zhang and McCall, 2012).

Ganglion cells. The ganglion cells are the output neurons of the retina and their long axons assemble in the optic nerve. Ganglion cells integrate all the information of the described upstream neurons and carry it to the downstream brain areas.

Ganglion cells receive glutamatergic input primarily from bipolar cells and express mainly ionotropic NMDA, AMPA and kainate receptors (Brandstatter et al., 1998; Massey and Miller, 1988) but might also express metabotropic glutamate receptors (Yang, 2004). As we have seen, ganglion cells receive inhibitory inputs from amacrine cells and express a diversity of GABAergic and glycinergic receptors.

Ganglion cells differ in their dendritic morphology and stratification into the inner plexiform layer (Fig.2.2). In the mouse retina morphological classification of ganglion cells estimate 15-20 different ganglion cell types (Coombs et al., 2006; Kong et al., 2005; Sumbul et al., 
2014; Volgyi et al., 2009). In the salamander retina 6-10 are described (Toris et al., 1995; Wang et al., 2016), however given the high diversity of bipolar and amacrine cells in the salamander retina, further types are expected. Similar to the other cell classes, it is not known which morphological type of ganglion cells expresses which receptor type.

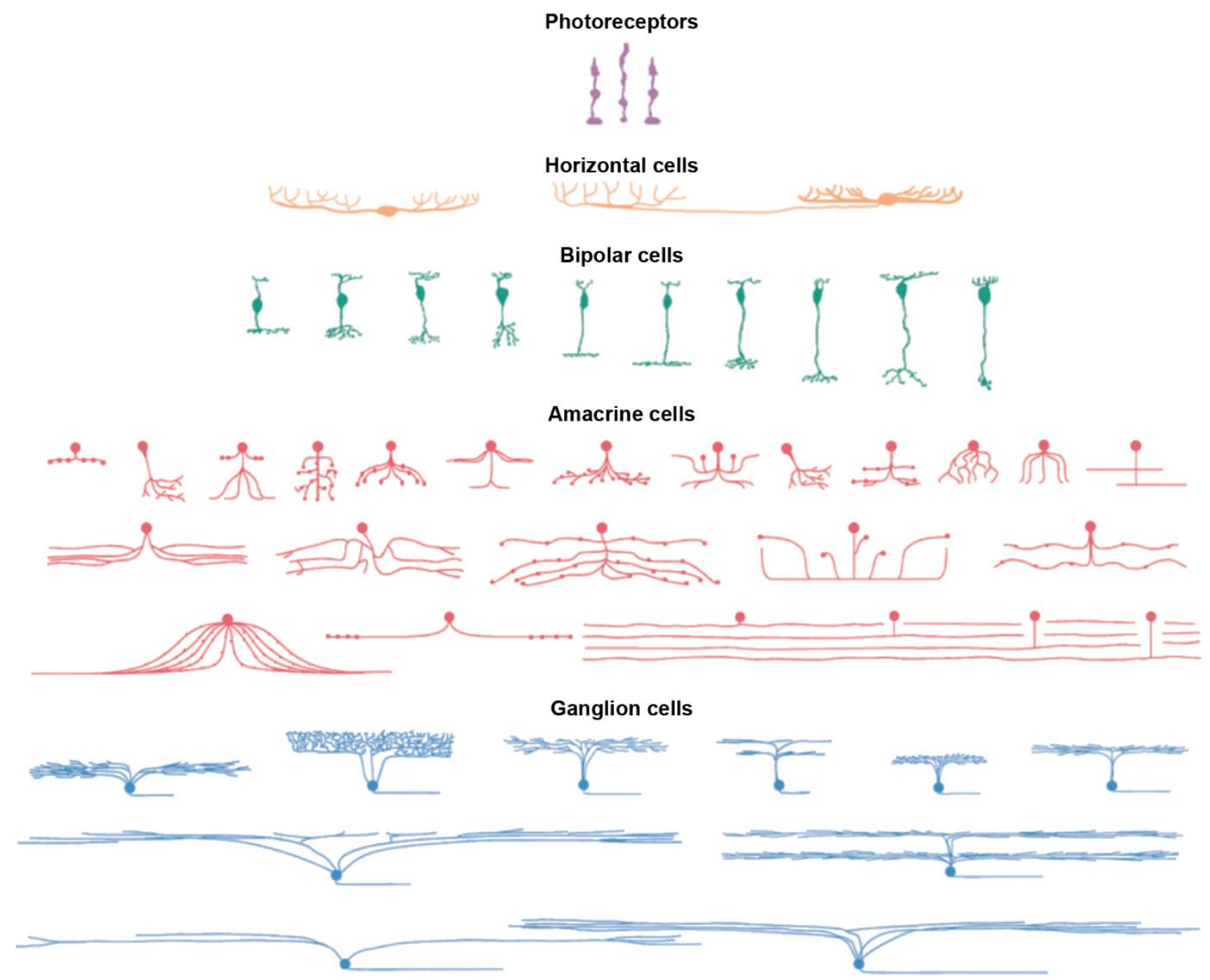

Figure 2.2. The five major cell classes and their subtypes in the retina. Image adapted from Masland (2001).

\subsection{General functional characterization in the retina}

We have seen a high diversity of morphology, receptors and neurotransmitters in the retina. Identifying these differences is an important first step in understanding the retina. Yet, to understand the function of the retina, we also have to study how the different neurons respond to light. In this subchapter, we view the classical physiological measurements of light responses such as the response polarity, center-surround structure and the temporal response properties. Further, we review how the previously introduced differences in morphology and receptor types 
manifest themselves physiologically. Moreover, we elucidate our described goals in the context of the literature.

\section{Response polarity}

In 1938, Hartline studied the light responses of single ganglion cell axons of the optic nerve of frogs with simple light bulbs (Hartline, 1938). He was among the first who studied light responses in the retina and discovered that some ganglion cells responded when the light was turned on (ON cells), others responded when the light was turned off (OFF cells). Further, he found ganglion cells that responded both when the light was turned on or off (ON-OFF cells). Thus, according to the light responses three functional classes emerged. However, how these preferences for light polarity come about remained unclear because intracellular recordings from cells distal to retinal ganglion cells were difficult to perform. Only around 30 years later, Werblin and Dowling (1969) recorded bipolar cell responses in the salamander retina and found two functional classes. One type of bipolar cell responded when the light was turned off (OFF cell), the other when the light was turned on (ON cell). Photoreceptors and horizontal cells were found to have only one polarity (OFF). Nearly a decade later, Famiglietti and Kolb (1976) showed that the ganglion cells inherit their light response preference from bipolar cells by making specific connections to bipolar cells with the respective response polarities. It took another $\sim 20$ years until the biochemical mechanism for the ON and OFF separation at the level of bipolar cells became clear. OFF bipolar cells express ionotropic glutamate receptors (iGluRs) (Brandstatter et al., 1997; Morigiwa and Vardi, 1999; Peng et al., 1995; Qin and Pourcho, 1996). Here, glutamate released by photoreceptors in darkness, opens the cation channels and depolarizes the cell. ON bipolar cells, on the other hand, express metabotropic glutamate receptors (mGluR6) (Nomura et al., 1994). Here, glutamate released from the photoreceptors closes cation channels and hyperpolarize the cell. The response polarity is a beautiful example of how the functional properties of the output ganglion cells emerge in the retina. Namely, through the unique interplay between synaptic connections, receptor types and cell classes. Yet, recently, it has been reported through imaging of calcium signals and glutamate release at the bipolar cell terminals, that some OFF bipolar cells, also exhibit delayed responses when the light is turned on (Baden et al., 2013a; Borghuis et al., 2013; Franke et al., 2017). These studies opened speculations about the presence of ON-OFF bipolar cells. Yet, they measured from the bipolar cell terminals, where the signals are intermingled with amacrine cells. In the current thesis, we record the bipolar cell signals at the soma. In our goal 1, we investigated the classical response characteristics like the polarity and we were curious whether we observe also ON-OFF bipolar cell responses. 


\section{Center-surround receptive field structure}

Hartline (1938), made another important contribution to the retina field. He introduced the term "receptive field", which is "the region of the retina which must be illuminated in order to obtain a response in any given fiber" (Hartline, 1938, p.410). Detailed studies by Kuffler (1953) further extended the concept of the receptive field, to a concentric center and antagonistic surround. He moved spots of light around the retina to find "receptors which feed into a single ganglion cell” (Kuffler, 1953, p.46). Curiously he discovered ON, OFF and even ON-OFF responses at different locations within the receptive field of the same cell. Further, he found that the different responses were organized into a central area sensitive to one polarity, a surrounding region responding to the opposite polarity and an intermediate region sensitive to both polarities. The findings of Kuffler (1953) are sometimes described under the term responsive surround; because the cell responds to surround stimuli of opposite polarity than in the center (Donner and Gronholm, 1984). In the same year, Barlow (1953) presented spots of different sizes to the retina ganglion cells and found that for some cells a further increase in spot size decreased the response. He coined the term inbibitory or suppressive surround; he speculated that retinal ganglion cells not just simply sum but also subtract signals over their receptive field (Barlow, 1953). It is not always clear if a cell possesses a suppressive surround, a responsive surround or both (Donner and Gronholm, 1984), nowadays the different effects are condensed into the term antagonistic surround.

The center-surround organization was also observed in bipolar cells. Werblin and Dowling (1969) found that the simultaneous illumination of center and surround reduces the response of the bipolar cells (suppressive surround). Yet, illumination of the surround alone, did not affect the cell (no responsive surround) (see also Thibos and Werblin (1978) for a similar finding). A bit later it was shown that an illumination of the surround can induce responses in the bipolar cells (responsive surround) (Burkhardt, 1974; Dacey et al., 2000; Fahey and Burkhardt, 2003). Interestingly, over the course of the years, not all studies observed an antagonistic surround in bipolar cells. For example, Berntson and Tylor (2000) recorded in dark-adapted mouse retinal slices and Borges and Wilson (1987) recorded in dark-adapted whole-mount salamander retinas, both studies did not observe an antagonistic surround (neither suppressive, nor responsive). It was speculated that the absence of the surround was either due to slice preparation, where the lateral connections are impaired, or to the low light level, where mainly rod bipolar cells are driven. Other studies (e.g. Hare and Owen (1990)) however also reported surround under low light levels. 
Surprisingly, even photoreceptors were found to show an inhibitory surround; illumination of the photoreceptor itself lead to a hyperpolarization, whereas a distal illumination of the surround produced a delayed depolarization (Baylor et al., 1971).

What could be the mechanism of the center-surround structure observed over the different cell types? Similar to the response polarity, it seems natural to deduce the antagonistic surround of ganglion cells from the center-surround organization in bipolar cells or photoreceptors (Donner and Gronholm, 1984). However, the mechanism seems to be more complicated and is still not fully understood. The center-surround organization in photoreceptors is explained through inhibitory feedback from horizontal cells onto photoreceptor terminals (Baylor et al., 1971; Sterling and Demb, 2004; Szikra et al., 2014). The cones in the far surround reduce the glutamate release onto horizontal cells, which then reduce their GABA release onto the central cones. The feedback mechanism of horizontal cells onto photoreceptors terminals might also be the origin for the center-surround organization in bipolar cells. For example, it was found that when the surround of cones was suppressed also the surround in bipolar cells disappeared (Mangel, 1991; Skrzypek and Werblin, 1983). However, other studies suggested a direct inhibition from horizontal cell onto bipolar cells through GABA (Sterling and Demb, 2004). Though this remains puzzling, only few studies reported GABAergic receptors on bipolar cell dendrites and it has been reported that the bipolar cell surround was neither GABAergic nor glycinergic (Hare and Owen, 1996). Recent studies suggest several mechanisms for the surround and the surround of different types of bipolar cells might be shaped by different synaptic circuits. For example, the feedback of horizontal cells onto cones, the direct feedforward mechanism from horizontal cells to bipolar cells, the feedback from amacrine cells and feedforward from amacrine to bipolar cells (Roska et al., 2000; Zhang and Wu, 2009). For ganglion cells, both horizontal cells (Mangel, 1991; Mangel and Miller, 1987) and amacrine cells (especially GABAergic wide-field) have been shown to be involved in forming the surround (Cook and McReynolds, 1998; Demb and Singer, 2015).

The center-surround structure is not as straightforward as the response polarity. In the present study, in goal 1, we therefore investigate the center-surround structure of bipolar cells and the influence of the surround on the response properties.

\section{Temporal properties}

Another important property of retinal cells is their temporal kinetics. Cleland et al. (1971) divided the responses of retinal ganglion cells into sustained and transient types. Transient cells responded very briefly to light, whereas sustained cells responded during the whole period of light stimulation. Transient and sustained responses were also found in bipolar cells, whereas 
photoreceptors and horizontal cells only exhibit sustained responses (Awatramani and Slaughter, 2000; Kaneko, 1970; Werblin and Dowling, 1969). Similar to the response polarity, the separation of the visual signals into transient and sustained channels was thought to originate from different glutamate receptor types at the bipolar cell dendrites. An elegant series of experiments performed by DeVries and colleagues in the ground squirrel showed that transient OFF bipolar cells express fast-adapting AMPA receptors, whereas sustained OFF bipolar cells express slow-adapting kainate receptors (DeVries, 2000; DeVries and Schwartz, 1999). However, the dichotomy remains controversial. A recent study in the mouse retina showed that both transient and sustained OFF responses were blocked by antagonists to kainate receptors but not AMPA receptors (Borghuis et al., 2014). It was speculated, that different kainate subunits alone could generate the diverse response kinetics (Lindstrom et al., 2014). Yet bipolar cells in the mouse were found to express AMPA receptors and kainate receptors (Puller et al., 2013). For the sustained and transient $\mathrm{ON}$ bipolar cells, which express metabotropic receptors, the molecular basis for the different temporal dynamics is even less understood. Possible mechanism are different metabotropic receptor subtypes, different proteins involved in the G- protein regulation, different sizes and rate of glutamate quanta release (Awatramani and Slaughter, 2000; Euler et al., 2014; Freed, 2000; Sterling and Demb, 2004).

It seems intuitive to ask whether the sustained and transient properties in ganglion cells are inherited from the bipolar cells (DeVries, 2000). Indeed there are some studies, showing that the sustained and transient responses in ganglion cells arise from the bipolar cells with the same temporal response dynamics (Awatramani and Slaughter, 2000; Roska and Werblin, 2001). However, other studies claim that amacrine cells shape, at least in part, the temporal response profile of retina ganglion cells (Dong and Werblin, 1998; Nirenberg and Meister, 1997). Thus, the jury is still on!

Similar to the response polarity, it was proposed that the neurons with different sustained-transient response types stratify at different depth of the inner plexiform layer. For example transient ganglion cells were found to ramify in the middle of the inner plexiform layer, while sustained ganglion cells ramify near the two nuclear boundaries (Roska et al., 2000). The full picture with bipolar cells is however more complicated. A recent study showed that although transient responses were observed more often in the middle layer, they could be found in other depths of the inner plexiform layer, too (Franke et al., 2017). Further, only the ON cells showed sustained responses (which ramify only at one nuclear layer closer to the ganglion cells). These results are different from another study of the same group, where the stratification agreed with the one proposed from ganglion cell recordings and where they found also OFF bipolar cells with sustained responses (Baden et al., 2013a). Further, both studies as well as studies in the 
salamander revealed that many bipolar cells display complex mixtures of sustained and transient components in their responses (e.g. transient peak with sustained plateau, slowly decaying, fast decaying, no decay). These findings are curious for the current thesis and to characterize the bipolar cell temporal properties we therefore investigate the sustained and transient component of their responses (goal 1).

\subsection{The role of nonlinearities in the retina}

In the previous section, we introduced the general response characterizations of retinal cells. Here, we investigate another response characterization, namely whether retinal neurons integrate light over their presynaptic inputs linearly or nonlinearly (spatial integration) and whether they transform light linearly or nonlinearly (output function).

\section{Nonlinear signal integration in retinal ganglion cells}

Many bipolar and amacrine cells connect to a single ganglion cell and form the centersurround structure of its receptive field. How a ganglion cell integrates these signals is a question that has puzzled the research in the retina since the beginning. Barlow (1953) for example investigated "the way in which a ganglion cell combines, or summates, effects contributed from different areas within this region (receptive field)" (p.71). He concluded that ganglion cells linearly add and subtract signals from different regions in the receptive field, but he also noted that there might be evidence that "the summation is not quite linear" (p.78). Yet, precise measurement of stimulus integration were lacking.

By a set of groundbreaking experiments, Enroth-Cugell and Robson (1966) introduced a new measure of signal integration within the receptive field. They recorded responses of cat retinal ganglion cells to sinusoidal gratings. The gratings were repeated at different positions, so that the receptive field center of the ganglion cell was covered successively by a fully dark, a fully bright and both bright and dark bars (Fig.2.3B). Enroth-Cugell and Robson (1966) were interested in the response of a ganglion cell to the grating position where both bright and dark bars where inside the receptive field center. Here, the change in luminance in one half of the receptive field was the exact opposite of the change in the other half (Enroth-Cugell and Robson, 1966). If a retina ganglion cell would do a linear summation, the two bars of opposite contrast would sum to zero and the cell would not respond. However, if the integration is nonlinear, the cell would respond to such a grating position. Enroth-Cugell and Robson (1966) indeed found cells that did not respond when the receptive field was covered by the two bars of opposite 
contrast (Fig.2.3A). They named those cells X-Cells. Curiously, they also found ganglion cells that clearly responded, when both bright and dark bars where inside the receptive field (Fig.2.3C). They named those cells Y-Cells. A bit later, morphological studies in the cat retina, suggested that the $\mathrm{X}$ and $\mathrm{Y}$ ganglion cells correspond to the anatomically distinguished beta (small dendritic field, X-Cell) and alpha (large dendritic field, Y-Cell) types (Boycott and Wassle, 1974). This finding gave rise to the idea, that different functional properties underlie different morphological cell types and started a "morphology reflecting function" paradigm in the retina (Saito, 1983).

Similar experiments were performed in various species and Y-type retinal ganglion cells have been documented among others in mouse (Carcieri et al., 2003; Krieger et al., 2017; Stone and Pinto, 1993), guinea pig (Demb et al., 1999), monkey (de Monasterio, 1978; Petrusca et al., 2007) and salamander retina (Bolinger and Gollisch, 2012). Further, the Y-type retinal ganglion cells were found to show fast ("brisk") and transient responses to light as well as large receptive fields (Boycott and Wassle, 1974; Cleland et al., 1971; Cleland et al., 1973; Crook et al., 2008; de Monasterio, 1978; Demb et al., 2001a; Petrusca et al., 2007). The X-type retinal ganglion cells on the other hand, were found to be slower ("sluggish") and more sustained with small receptive fields (Boycott and Wassle, 1974; Cleland et al., 1971; Cleland et al., 1973; de Monasterio, 1978). However, as implied by some of the mentioned studies (e.g. de Monasterio (1978)), the full picture seems to be more complicated than a strict dichotomy (for example the terms W-cells or Q-cell were introduced (Stone and Hoffmann, 1972; Troy et al., 1995), for a historical description about the terminology see also Cleland and Levick (1974) or Crook et al.(2008). Nowadays, the term "Y-cell" and "X-cell” is mainly used to describe the property of nonlinear or linear summation inside the receptive field independent of the morphology and other response properties. More recent studies for example documented nonlinear retina ganglion cells with slow (“sluggish”) responses (Demb et al., 1999; Demb et al., 2001a), with sustained responses (Krieger et al., 2017) and variable receptive field sizes (e.g. Heine and Passaglia (2011) did not find a difference in the receptive field size between linear and nonlinear cells, further Freeman et al. (2015) found nonlinear spatial summation in the small OFF midget ganglion cells of primates). It seems that nonlinear spatial summation is a property observed in different types and sometimes even all recorded retinal ganglion cells show nonlinear spatial summation (Bolinger and Gollisch, 2012; Demb et al., 1999). Another study showed that the degree of nonlinear spatial integration follows a continuum and not a strict dichotomy (Carcieri et al., 2003).

It was proposed, that linear $\mathrm{X}$-type retinal ganglion cells report the average light level inside their receptive fields, while spatially nonlinear $\mathrm{Y}$ - retinal ganglion cells extract patterns with high spatial structure or small high-contrast objects (Demb et al., 2001a; Gollisch, 2013). 
A

$X$ cell (linear)

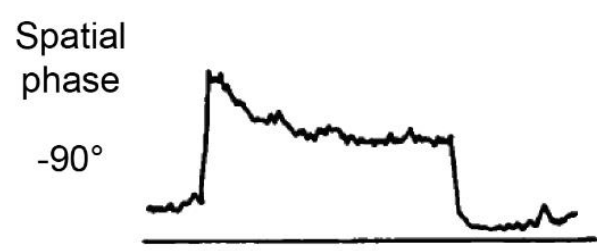

$-0^{\circ}$

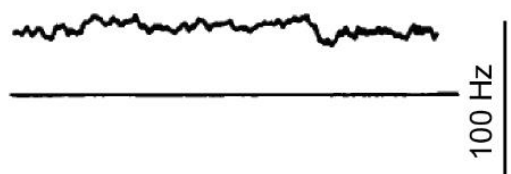

$90^{\circ}$

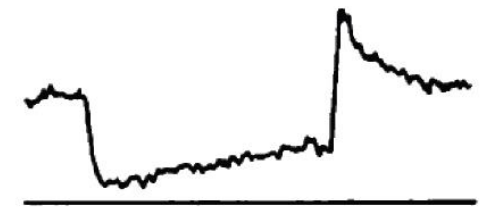

$180^{\circ}$

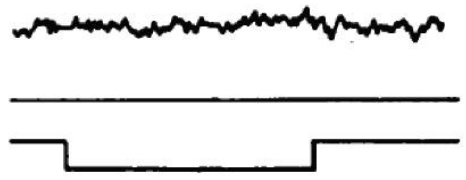

B
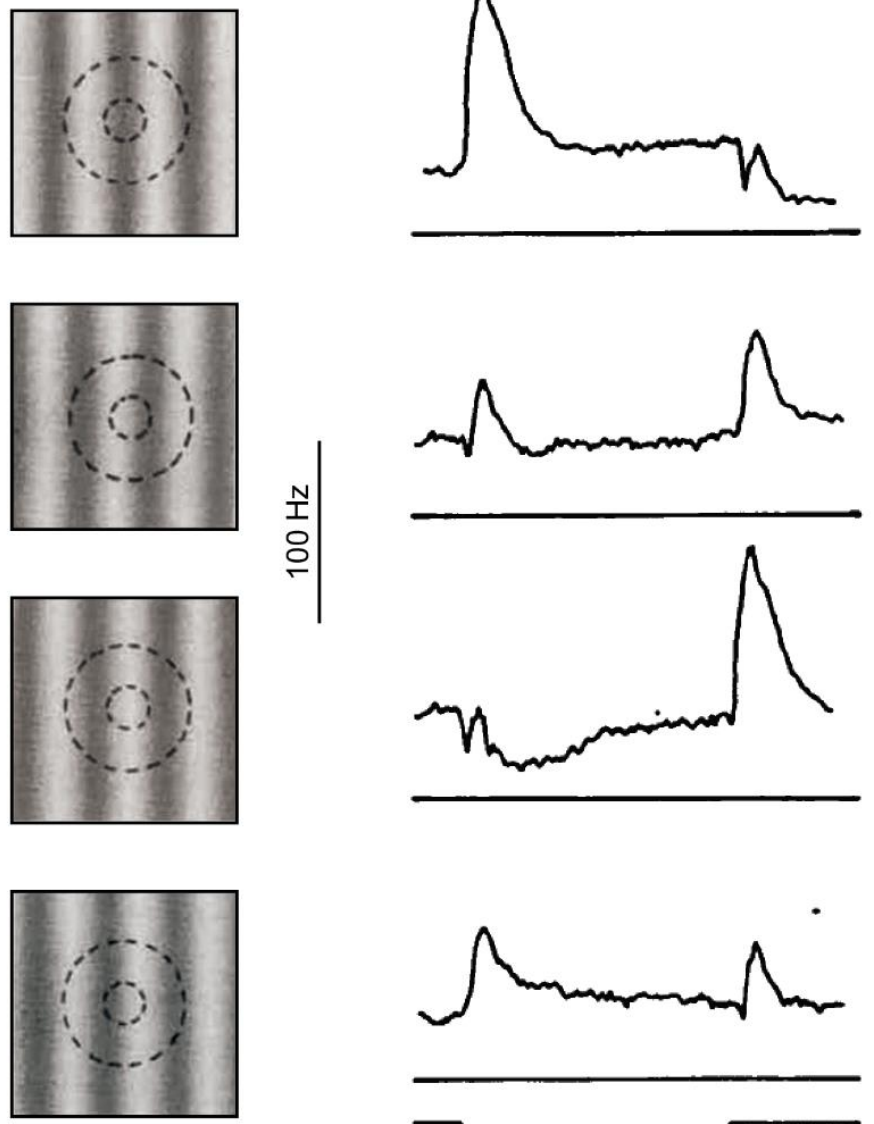
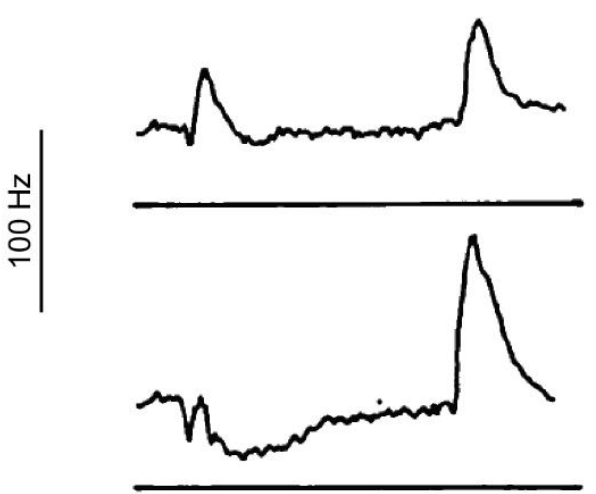

$Y$ cell (nonlinear)
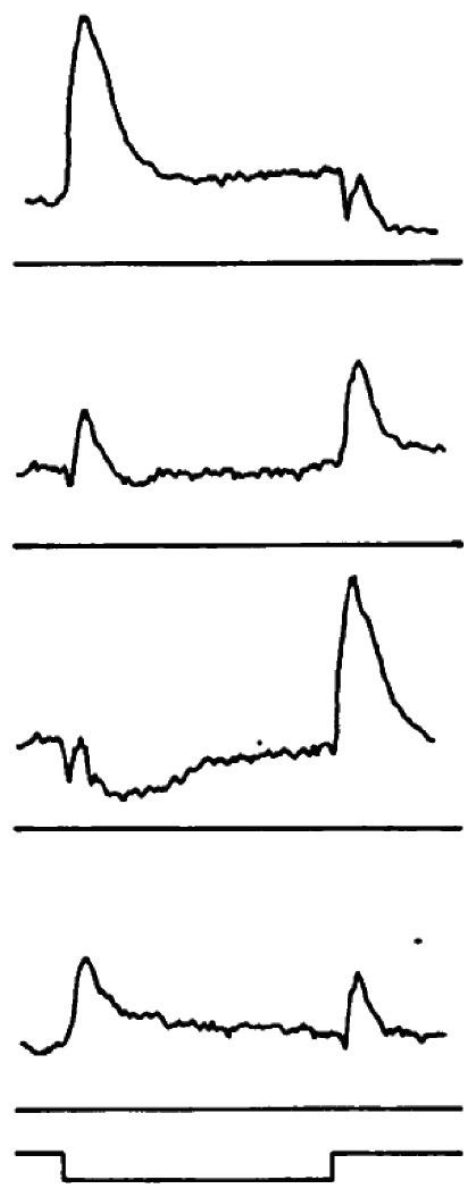

Figure 2.3. Spatial integration in retinal ganglion cells. A. Response traces of a linear retinal ganglion cell (X cell) that does not respond to the grating when both white and black bars cover the receptive field $\left(0^{\circ}\right.$ and $\left.180^{\circ}\right)$. Yet, when the black bar covers the receptive field $\left(-90^{\circ}\right)$ the cell clearly responds. B. The position of the stimulus pattern in relation to the receptive field location is shown. C. Response traces of an example nonlinear ganglion cell (Y cell) that responds when both white and black bars cover the receptive field $\left(0^{\circ}\right.$ and $\left.180^{\circ}\right)$. The image is adapted from Enroth-Cugell and Robson (1966) and Enroth-Cugell and Robson (1984).

\section{Role of bipolar cell output nonlinearity for signal integration in ganglion cells}

What could be the mechanism behind the observed linear and nonlinear integration in retina ganglion cells? The linear mechanism has been attributed to a linear signal transmission from photoreceptors to bipolar cells to X-type retinal ganglion cells (Hochstein and Shapley, 1976a, b; Victor and Shapley, 1979; Victor et al., 1977). The idea was supported by diverse experiments, showing that photoreceptors and bipolar cells respond approximately linear to light (Baylor et al., 1974; Naka et al., 1987; Sakai and Naka, 1987a, b; Toyoda, 1974). Recent studies further confirmed a linear relationship between light intensity and voltage (Baccus and Meister, 2002; Dacey et al., 2000; Fahey and Burkhardt, 2003; Rieke, 2001). The mechanism of a linear 
summation from an X-type retinal ganglion cell is illustrated in Figure 2.4A. The OFF X-type retinal ganglion cell sums the signals over the connected linear bipolar cells. When presenting a grating stimulus, some of the connected OFF bipolar cells (red cells) are exposed to the black bars and thus depolarize, other bipolar cells (blue cells) are exposed to the white bars and hyperpolarize (Fig.2.4A). The amount of hyper- and depolarization are equal. Thus, when the ganglion cell sums the input signals from the red and blue bipolar cells, the signals cancel out and the ganglion cell is silent. It is important to mention that linear integration not only requires linear light-voltage relationship but also linear synaptic transmission (i.e. symmetric increase and decrease in neurotransmitter release). Linear synaptic transmission needs a high-sustained rate of neurotransmitter release at the synapses of photoreceptors to bipolar and bipolar to ganglion cells (Schwartz and Rieke, 2011). The ribbon synapses present in both photoreceptors and bipolar cells are the candidate for linearity (Shapley, 2009). Photoreceptors and ON bipolar cells were found to have relatively high baseline release of glutamate and minimal rectification (Borghuis et al., 2013; Manookin et al., 2008; Thoreson et al., 2003; Witkovsky et al., 1997; Zaghloul et al., 2003).

Nonlinear spatial integration of the Y-type retinal ganglion cells has originally been attributed to amacrine cells (Hochstein and Shapley, 1976a; Sakai and Naka, 1987a, b; Victor and Shapley, 1979; Victor et al., 1977). Many amacrine cells have been found to show nonlinear responses to light (Freed et al., 1996; Sakai and Naka, 1987a, b). Some amacrine cells even generate spikes (Stafford and Dacey, 1997; Werblin and Dowling, 1969), which is an epitome for nonlinear voltage responses. However, it was difficult to grasp how inhibitory amacrine cells could generate an activation in retina ganglion cells to a grating (Demb et al., 2001a). In a series of experiments, Demb and colleagues investigated this question and discovered that when the amacrine cell inputs are blocked, the nonlinear response of the Y-type retina ganglion cell to a central grating persists (Demb et al., 2001a). They concluded that the nonlinear spatial summation is driven by an array of nonlinear bipolar cells (output function) and that the nonlinear response must be intrinsic to the bipolar cell (Demb et al., 2001a). The proposed mechanism of a nonlinear summation from a Y-type retinal ganglion cell is illustrated in Figure 2.4B. The OFF Ytype retinal ganglion cell sums the signals over the connected nonlinear bipolar cells. When presenting a grating stimulus, some of the connected OFF bipolar cells (red) are exposed to the black bars and thus strongly depolarize, other bipolar cells (in blue) are exposed to the white bars and remain silent (Fig.2.4B). Thus, here the input signals show different amount of hyper- and depolarization. Therefore, the summation of the bipolar cell inputs does not cancel at the level of the ganglion cell and cause the Y-type ganglion cell to respond. Demb and colleagues did not directly record the rectified signal from bipolar cells. Yet, their idea gained support by diverse 
studies performing single cell recordings from bipolar cells' somas and reported nonlinear responses (Burkhardt and Fahey, 1998; Euler and Masland, 2000; Fahey and Burkhardt, 2003). Burkhardt and Fahey (1998) for example showed that for some cells the increase in activity to the preferred contrast (e.g. white) is more than the decrease in activity to a non-preferred contrast (e.g. black). Furthermore, studies that imaged the glutamate release at bipolar cell terminals (Borghuis et al., 2013) and studies that measured input currents to ganglion cells showed clear rectification (Molnar et al., 2009; Zaghloul et al., 2003). Besides, fast transient almost spike-like events were observed at the axon terminals of bipolar cells (Baden et al., 2013a; Franke et al., 2017).

The observed nonlinear signals in bipolar cells contradict the findings of linear bipolar cells that were proposed to be responsible for X-type retinal ganglion cells. The inconsistency between different studies has been attributed to different stimulus dynamics and studied bipolar cell types (Burkhardt and Fahey, 1998; Schwartz and Rieke, 2011). For example, Burkhardt and Fahey (1998) showed contrast steps, while Rieke (2001) showed a white-noise contrast sequence. Therefore, we studied in this thesis the output function in bipolar cells by presenting both, contrast steps and white-noise stimuli. Further, we investigate whether linear and nonlinear light responses are properties of specific bipolar cell types (e.g. sustained vs. transient).

Curiously, previous studies have mainly focused on how retinal ganglion cells integrate signals over bipolar cells. However, how bipolar cells integrate signals over photoreceptors has been largely neglected. Bipolar cells are assumed to perform a linear integration over the photoreceptor signals, mainly due to the reported linear light responses in photoreceptors (Baccus and Meister, 2002; Baylor et al., 1974; Rieke, 2001). However, evidence for nonlinear neurotransmitter release in photoreceptors is challenging the view of a linear integration in bipolar cells. For example nonlinear responses have been reported in rods (Dunn and Rieke, 2008; Field and Rieke, 2002; van Rossum and Smith, 1998) and in cones (Baden et al., 2013c; Dunn et al., 2007). To solve this puzzle, we performed similar experiments that have been used to find nonlinear integration in ganglion cells, on bipolar cells (goal 2). 
A

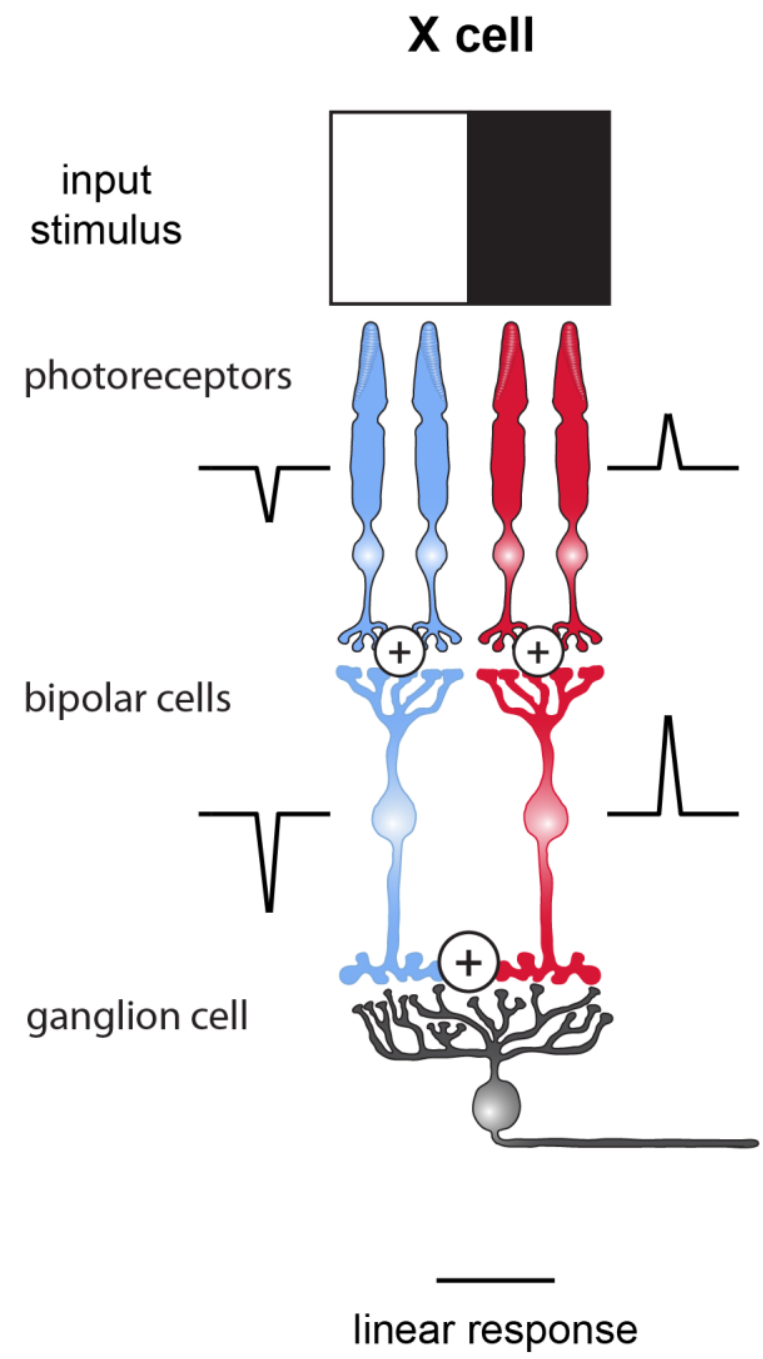

B

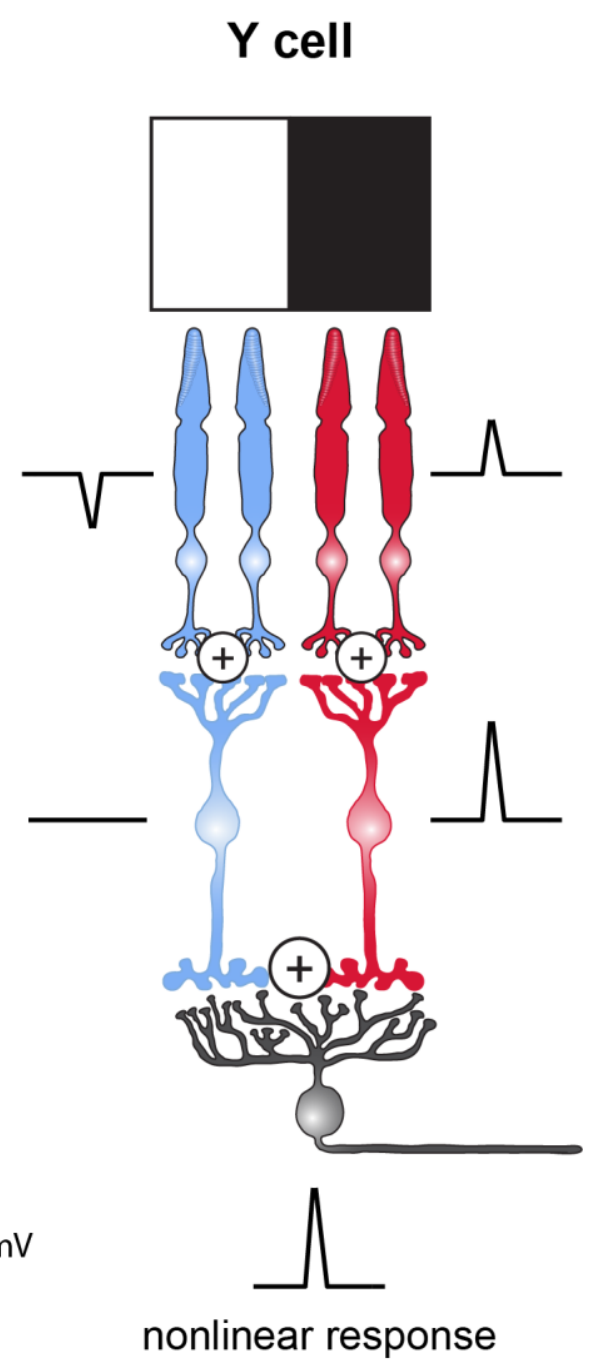

Figure 2.4. Mechanism bebind linear and nonlinear spatial integration in ganglion cells. A. Stimulus with half white and half black is shown to the photoreceptors. The blue photoreceptors hyperpolarize and the red photoreceptors depolarize by the same amount. The blue photoreceptors send their signals to the blue bipolar cells and the red photoreceptors to the red bipolar cells. The signals are summed by the bipolar cells, which respond with equal amount of hyperpolarization and depolarization. The ganglion cell sums the two signals from the blue and red bipolar cells to zero and does not respond to the stimulus (linear response). B. In contrast to A, the blue bipolar cell does not hyperpolarize to light but stays silent, while the red bipolar cell depolarizes. The ganglion cell sums the two signals and respond to the stimulus because the two input signals do not cancel (nonlinear response). The figure was inspired by Demb et al. (2001a). 


\subsection{Computational models in the retina}

We have seen a high diversity of cell types (subchapter 2.1) and functional properties (subchapter 2.2 and 2.3) in the retina. Computational models provide useful information to bridge the gap between the different cell types and the observed functions (for reviews see Baccus (2007), Field and Chichilnisky (2007) or Gollisch and Meister (2010). Currently, we cannot record from all neurons in the retina, thus we rely on mathematical models that provide us with intuition about how neurons combine and process inputs to generate a functional diversity. One of the most important models to understand the function of the retina is the linear-nonlinear model (LN-model) (Chichilnisky, 2001; Gollisch, 2013).

\section{Linear-Nonlinear Model}

The linear-nonlinear model is a cascade model that comprises two processing steps (Baccus, 2007). First, the input stimulus is linearly integrated by a filter. Second, the filtered output is passed through a nonlinear transformation. The idea of the model is that neurons have internal filters, and the filters define which stimulus features are enhanced and which ignored. To picture neurons as filters is one of the main "paradigms" in sensory neuroscience and is grounded in diverse findings (e.g. ON, OFF cells, receptive fields, motion or color sensitivity, famous "grandmother cell" or "bug detector"). It is believed that neurons gather information that is behaviorally significant while discard other information and that different neurons enhance different features of the environment (Simmons and Young, 1999). In this context, if we stimulate the sensory system with inputs, the neurons transmit only certain aspects of the input signal i.e. those inputs that "pass through the filter" (1. stage of the NL-model). For example, the neuron in Figure 2.5A only likes stars and stimuli that have a similar star shape pass the neuron's filter. However, the inputs that "pass through the filter" do not necessary have to activate the neurons. Many neurons show additional internal nonlinearities (2. stage of the NL-model). For example, to generate an action potential a threshold has to be passed (i.e. depolarization reaches $\sim 50-55 \mathrm{mV}$ ). Thus, in our example, small stars might not activate the neuron (Fig. 2.5A, output). Moreover, neurons often show response saturation; to exemplify, at a certain point, a further increase in the size of the star does not increase the response further.

To implement the linear-nonlinear model, one, in a first step, has to figure out the neuron's filter. The introduced characterization in subchapter 2.2 and 2.3 (e.g. spots of light and gratings) is one way to describe the sensitivity of neurons. Here, however, the difficulty is to translate the responses into a concrete mathematical filter. An alternative method is based on white-noise analysis (Chichilnisky, 2001; Gollisch, 2013). Here, the input stimulus can for 
example be a uniform randomized contrast sequence with no spatial structure or multiple contrast sequences that are shown simultaneously at multiple small areas (Fig.2.5B). The filter is readily obtained by correlating the response with the stimulus (see methods for mathematical details). Figure 2.5B shows an example of an obtained filter. The neuron in Figure 2.5B responds to light stimuli that are restricted to an area in space (seen in the black contrast of the pixels). Light falling outside the restricted area (on the gray pixels), does not affect the neuron's response. The filter can also be called spike-triggered average (STA) or voltage-triggered average (VTA), depending whether the action potential or voltage signal is measured as a response.

The input stimulus signal is then passed through the determined filter. At this stage, the influence of light signals presented outside the receptive field is reduced, while light signals presented inside the receptive field are enhanced. Yet, as we have indicated, the filtered stimuli does not necessarily have to activate a neuron. For example, increasing the light level by a factor of one million does not increase the membrane potential in a kilovolt range (saturation) (Baccus, 2007). Further, very small inputs, for example of one single photon, give usually rise to little or no outputs (threshold). Therefore, a second step in the linear-nonlinear model was introduced, termed the nonlinearity, which accounts for cell intrinsic dynamics like response threshold and saturation (Chichilnisky, 2001). Figure 2.5C shows such a classical nonlinear input-output transformation of neurons. Yet, the experiments in the retina are usually not performed under the full stimulus range (all possible light intensities), but under a small i.e. more naturalistic range of intensities (see red inset in Fig.2.5C). In this stimulus regime, the neurons exhibit diverse nonlinear transformations, which indeed can differ from the classical threshold and saturation nonlinearity. Beyond the threshold, the neuron can show for example a linear transformation i.e. the spike rate is proportional to the input (threshold-linear), they can show quadratic increase i.e. the spike rate increases more strongly than the input (threshold-quadratic) or further, they can exhibit a saturation i.e. the spike rate increases less than the input (Fig.2.5C). Most retinal ganglion cells exhibit such types of nonlinearities (threshold-linear, threshold-quadratic, threshold-saturation). Photoreceptors, bipolar and horizontal cells are generally approximated by a linear input-output transformation, see Figure 2.5C. It is important to mention that retinal ganglion cells with high spontaneous activity can show similar linear transformation i.e. they increase and decrease their spike rate with similar amounts from a high spontaneous activity (Schwartz and Rieke, 2011).

\section{Response prediction with the linear-nonlinear model}

Besides the functional description of the input-output transformation (i.e. type of nonlinear transformation), the nonlinearity provides a "lookup table". We can read i.e. predict, 
how the neuron responds to different sets of stimuli. For example, an input stimulus that matches the filter is mapped to a high neuronal response (y-axis) (Fig.2.5B, red dashed rectangle). On the other hand, a stimulus with opposite polarity to the linear filter is mapped onto a neuronal response close to zero (Fig.2.5B, blue dashed rectangle). Thus, once we know the filter and the output function of a neuron, we can predict responses to new stimuli. Figure 2.5B shows a predicted trace of a neuron in red.

How accurate does the linear-nonlinear model, describe the light responses of neurons? This question has been investigated in the retina almost exclusively for ganglion cells. Three different types of stimuli are traditionally used to probe the linear-nonlinear model: 1. artificial uniform contrast flicker (full-field white noise), 2. artificial contrast flicker at multiple location (spatio-temporal white noise) and 3. natural stimuli (see Fig.2.5B, inputs). For uniform contrast flicker stimuli, the predicted traces of the linear-nonlinear model are found to accurately match the actual responses of the retinal ganglion cells. For example matches of above $70 \%$ have been reported (Chichilnisky, 2001; Pillow et al., 2008; Zaghloul et al., 2003; Zaghloul et al., 2007), but one study also reported less precise prediction (e.g. matches $\sim 43-84 \%$ ) for some cells (Pillow et al., 2005). For stimuli with spatial structures, like the spatio-temporal white noise and natural stimuli, the linear-nonlinear model yields much diverse and often less accurate description of retinal ganglion cells' responses. For example, studies using spatio-temporal white noise reported matches of $\sim 10-60 \%$ (Liu et al., 2017), 40-100\% (Heitman et al., 2016) ${ }^{4}$ or $\sim 10-60 \%$ (Freeman et al., 2015). Studies investigating the model accuracy with natural stimuli reported matches of $\sim 30-90 \%$ (Liu et al., 2017), 0-80\% (Heitman et al., 2016) or 20-100\% (Turner and Rieke, 2016).

The linear-nonlinear model was mainly used to study retinal ganglion cells' responses. It has never been tested how accurate the linear-nonlinear model can predict bipolar cells' responses to stimuli with spatial structure. Thus, in our goal 3, we studied how accurate the linearnonlinear model can predict bipolar cell responses and probed the same three types of stimuli that are traditionally used for ganglion cells (see again Fig. 2.5B).

\footnotetext{
${ }^{4}$ Heitman et al. (2016) test the prediction performance with a generalized linear model (GLM). GLM is an extension of the LN-model. Additional to the linear filter and nonlinearity, the GLM includes a post-spike feedback that takes into account spike bursts and refractory period.
} 
A

input

filter

output

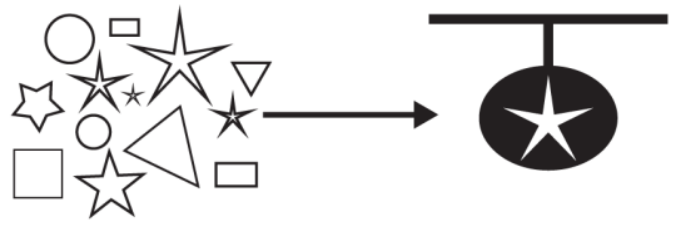

B

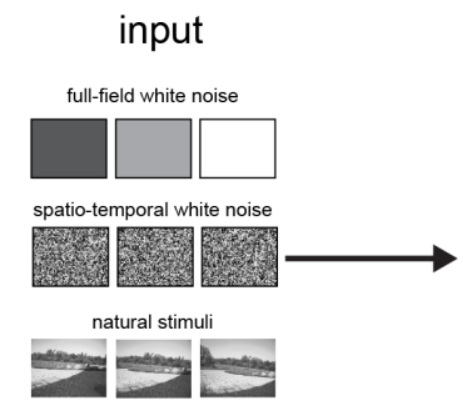

neuron

filter
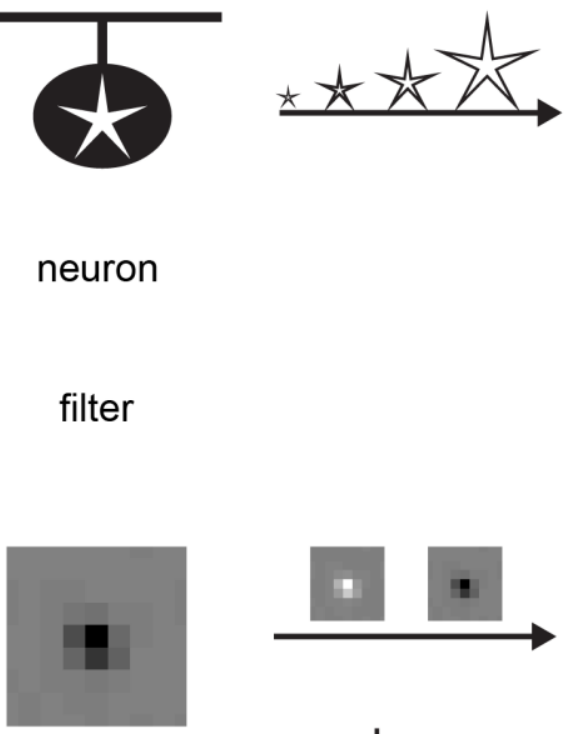

neuron

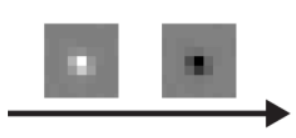

20

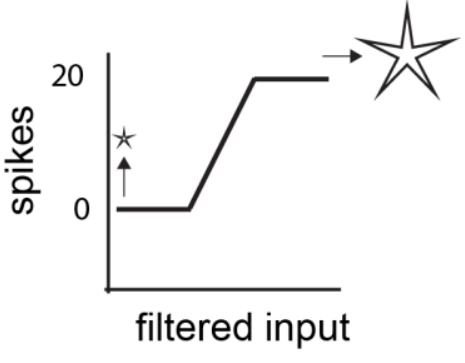

output

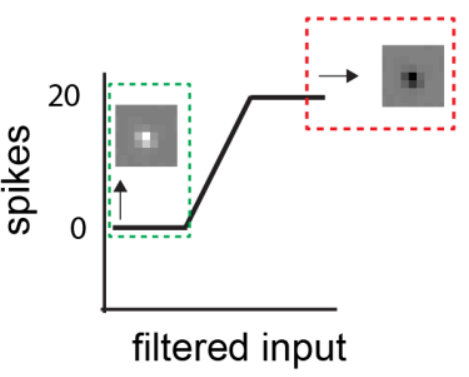

— prediction

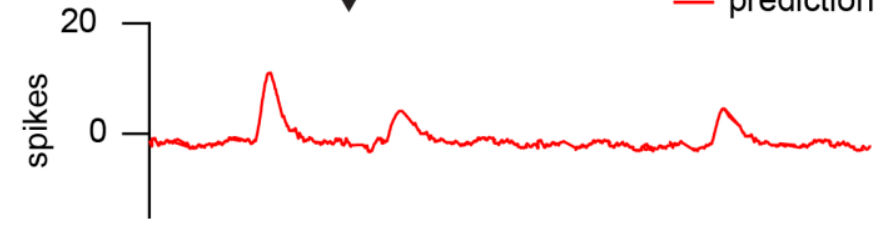

C

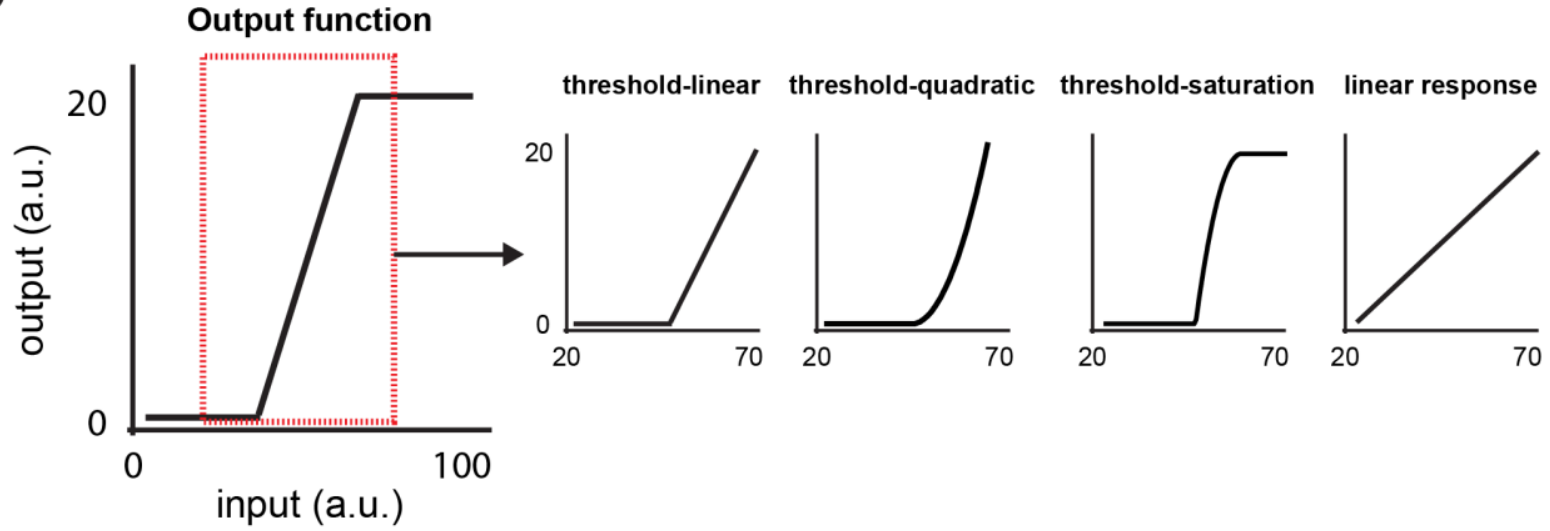

Figure 2.5. Linear-nonlinear model. A. Simplified illustration of the linear-nonlinear model (LN-model). The input stimulus are different geometrical shapes. The neuron has a filter in form of a star (1. stage of the model). Input stimuli with a star shape can pass through the filter while triangles or circles do not influence the neuron. The filtered stimulus is then passed through a cell intrinsic nonlinear transformation (e.g. spike threshold and response saturation) to generate a response in the cell (2. stage of the model). B. The classical input stimuli used in experiments in the retina are illustrated. Further, a spatial filter of a real neuron is shown. If the input signal matches the filter (red dashed rectangle), the neuron responds to the stimulus, if however the signal is opposite to the filter (blue dashed rectangle) the neuron is silent. From the output function, a prediction of a neurons response can be generated (red trace). C. Examples of 
output functions. Over a full stimulus range, neurons exhibit nonlinear output functions in form of a threshold and a saturation. Yet, in our experiments, we limit the stimulus range (red dashed rectangle). Here, the transformation can show diverse shapes, for example threshold-linear, threshold-quadratic, threshold-saturation or fully linear. 



\section{Methods}

In this chapter, we provide the experimental and analytical methods. First, we introduce the animal model (subchapter 3.1) and the retina tissue preparation (subchapter 3.2). Then, we describe the technique to simultaneously record from bipolar and ganglion cells (goal 4, subchapter 3.3) and we show how we identified bipolar cells (subchapter 3.4). Finally, we describe the analysis performed for the general response characterization (goal 1), the identification of nonlinearities (goal 2) and the linear-nonlinear model (goal 3).

\subsection{Animal model}

The experiments were done with retinas from the axolotl salamander (Ambystoma mexicanum, pigmented wild type, either sex). The axolotl is an amphibian that becomes sexually mature without undergoing a metamorphosis, thus they remain aquatic. Why using salamanders for vision research?

The use of amphibian in vision has a long tradition. For example, the concepts of response polarity and receptive field were first described in axons of ganglion cells in the frog (Hartline, 1938). Yet, recordings were mainly performed at the optic nerve and intracellular recordings from cell bodies in the retina were difficult because the micropipettes fail to penetrate the small cell bodies. In a series of experiments, Bortoff and colleagues showed that the mudpuddy salamander retina (Necturus maculosus) is ideal for intracellular recordings because of its large cell bodies (Bortoff, 1964; Bortoff and Norton, 1965). Therefore, the mudpuddy salamander became an important animal model to study the retina and was used for groundbreaking characterizations of bipolar cells' responses to light (Werblin and Dowling, 
1969). The tiger salamander (Ambystoma tigrinum) is another important retina model that is commonly used to study the retinal circuitry. For example, a high morphological diversity has been discovered in bipolar cells of the tiger salamander retina (Wu et al., 2000). Further, the tiger salamander retina is an important model to study functional properties of the retina like contrast adaptation (Baccus and Meister, 2002) or object motion (Ölveczky et al., 2003). The axolotl salamander is another salamander animal model used to study diverse response characteristics in the retina, for example membrane potential properties of bipolar cells (Attwell et al., 1987), direction-selective ganglion cells (Kuhn and Gollisch, 2016) or nonlinear spatial integration (Bolinger and Gollisch, 2012). Tiger, mudpuddy and axolotl salamanders are close relatives and no differences in the organization among their retinas are reported. The decision between the different salamander species depends on local availability and therefore in our lab we use axolotl salamanders.

Recently it became more and more common to study the retina and visual system of the mouse. Mainly advances in genetic tools made the mouse an interesting animal model. One may naturally wonder whether the findings in the amphibian retina can be transmitted to the mouse retina. Both, mammalian and amphibian retinas show a common architecture and most cell properties reported in the amphibian retina were found in the mouse retina too. Thus, even though amphibians are not mammals, they still provide a promising animal model to study the retina. We decided to perform our experiments on the salamander retina because their large cell bodies made it possible to perform intracellular recordings with sharp microelectrodes for more than an hour. The long recordings were needed for some of the questions studied in this thesis. We also performed bipolar cell recordings in the mouse retina, yet here, the recording durations were between 10-30 minutes (data not shown). Further, the rate of successfully impaling a bipolar cell was higher in the salamander retina.

\subsection{Tissue preparation}

All experiments were performed in the dark to avoid bleaching of the photoreceptors. The salamanders were anesthetized in ice water and dark-adapted for $\sim 1 \mathrm{~h}$. Then, the animals were decapitated and pithed to stop reflexes. The eyes were enucleated and immediately put into an oxygenated Ringer's solution $\left(110 \mathrm{mM} \mathrm{NaCl}, 2.5 \mathrm{mM} \mathrm{KCl}, 1 \mathrm{mM} \mathrm{CaCl} \cdot 2 \mathrm{H}_{2} \mathrm{O}, 1.6 \mathrm{mM}\right.$ $\mathrm{MgCl}_{2} \bullet 6 \mathrm{H}_{2} \mathrm{O}, 22 \mathrm{mM} \mathrm{NaHCO}, 10 \mathrm{mM} \mathrm{D-Glucose} \mathrm{monohydrate,} \mathrm{continuously} \mathrm{bubbled} \mathrm{with}$ Carbogen $\left(95 \% \mathrm{O}_{2}\right.$ and $\left.\left.5 \% \mathrm{CO}_{2}\right), \mathrm{pH} \sim 7.4\right)$. The dissection of the eye was done under infrared light, with the help of infrared goggles installed on a microscope. The eyes were opened along the 
cornea to access the retina. The lens and vitreous humor were removed and the retina was slowly detached from the pigmented epithelium. Once fully detached, the retina, with a diameter of $\sim 3 \mathrm{~mm}$, was taken out of the eye. To mount the retina into the setup, we used a nitrocellulose filter membrane (Fig.3.1A). In short, we made a hole of $1.5-2 \mathrm{~mm}$ in diameter into the filter membrane with a biopsy punch. We centered the retina over the hole and slightly pressed the edges of the retina onto the filter membrane. The retina on the filter paper was mounted onto the multielectrode array in a recording chamber inside the setup. The ganglion-cell side faced the electrodes and the photoreceptors faced up. The retina was continuously superfused with oxygenated Ringer's solution at room temperature $\left(\sim 25^{\circ} \mathrm{C}\right)$.

\subsection{Simultaneous recordings from bipolar and ganglion cells}

A schema of the combined recording technique we developed as our goal 4 is illustrated in Figure 3.1B. The setup is composed of three main parts: sharp microelectrode, multielectrode array and the light stimulation. The idea of the combined recording setup comes from several studies in the group of Markus Meister (Asari and Meister, 2012, 2014; de Vries et al., 2011). Our approach is similar to their combined recording technique, yet we performed some modifications. In this section, we are going to describe each of the three parts separately. 
A
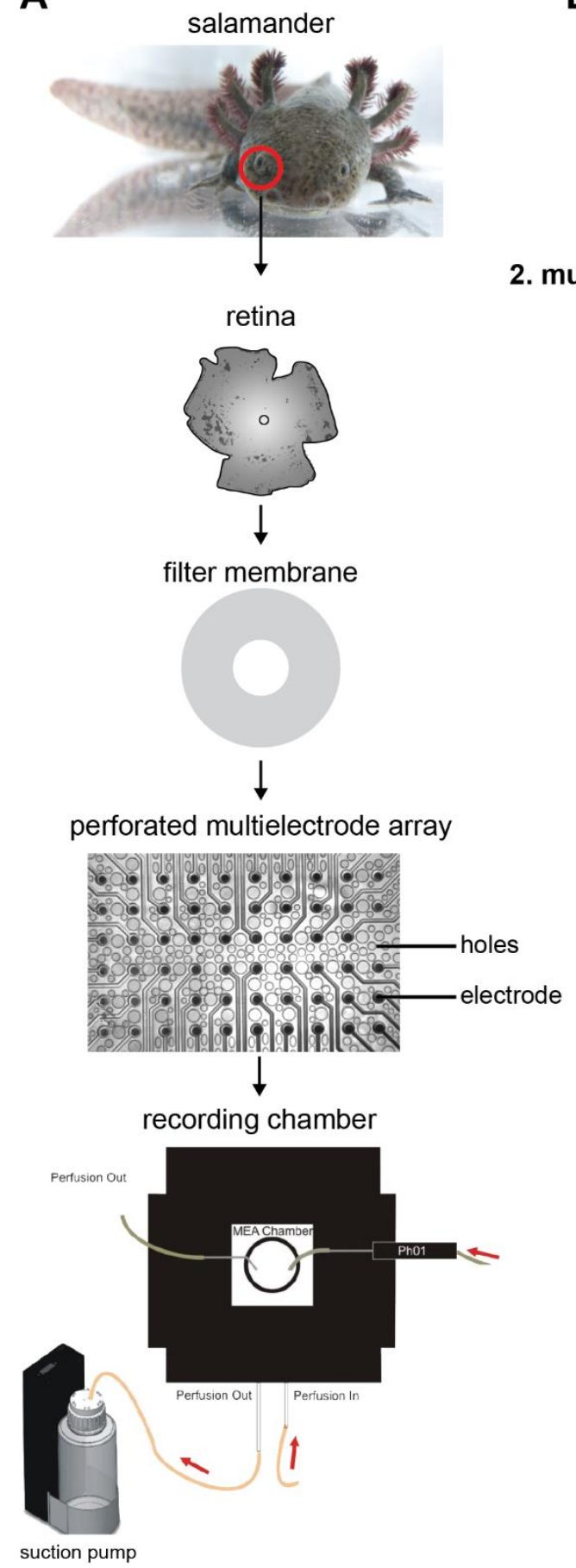

B
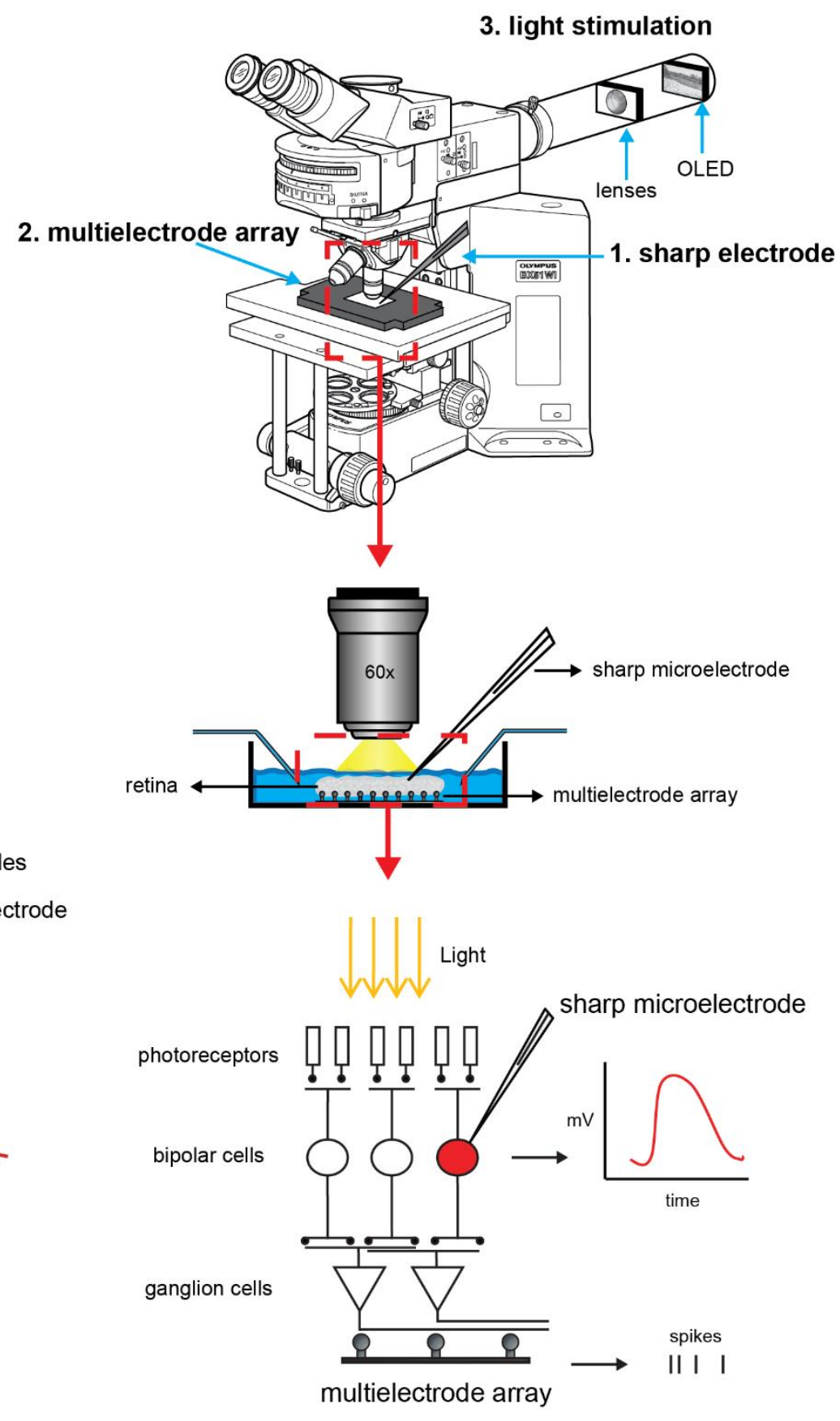

Figure 3.1. Combined recording technique (goal 4). A. The retina of the salamander is prepared and mounted onto a filter membrane. Then, the filter membrane is put onto a perforated multielectrode array (pMEA) so that the retinal ganglion cells face the electrodes. The perforated MEA has small holes between the electrodes that allow applying slight suction controlled by a suction pump. B. Microscope with the three main parts of the combined recording technique: 1. sharp electrodes, 2. multielectrode array and 3. light stimulation. The sharp electrode was inserted from the photoreceptor layer to the bipolar cells and the depth of the electrode was monitored with a $60 x$ objective. The bipolar cell signal was simultaneously recorded to the spiking activity of multiple ganglion cells. The light was projected through the objective onto the photoreceptor layer. Image of the recording chamber with permission from Multi Channel 
Systems MCS GmbH, taken from the Constant Vacuum Pump (CVP) manual (2017). Image of the Olympus microscope (BX51Wl), taken from the Olympus instruction BX51Wl fixed-stage upright microscope manual (2009). Picture of the salamander and perforated multielectrode array taken by Norma Kühn and myself in our lab.

\subsubsection{Sharp microelectrodes}

The bipolar cell's signals were recorded with sharp microelectrodes. We chose sharp over patch electrodes for several reasons. The sharp microelectrodes have a very fine tip and therefore allow penetrating the whole-mount retina from the photoreceptor side without seriously damaging the tissue. Further, they allow for fairly long recording periods (up to 1 hour, maximum 2h) without the problem of dialysis of the intracellular content as seen in whole-cell patch-clamp recordings. Various important findings about bipolar cell response properties come from recordings with sharp microelectrodes and the technique has been shown suitable for the intended experiments (Asari and Meister, 2012, 2014; Baccus and Meister, 2002; Burkhardt and Fahey, 1998; Fahey and Burkhardt, 2003; Ölveczky et al., 2003; Werblin and Dowling, 1969).

The construction of sharp microelectrodes, that allowed long and stable recordings, was much harder than originally conceived. We used the P-97 micropipette puller (Sutter Instruments, Novato, CA). The aim was to construct microelectrodes with very fine tips that can successfully penetrate the cell bodies. As a rule of thumb, the resistance roughly reflects the diameter size of the tip of the sharp microelectrode. The higher the resistance is of the electrode the smaller the tip diameter size. We tried to get high resistance electrodes of $>100 \mathrm{M} \Omega$ that have approximated tip sizes of $\sim 0.06 \mu \mathrm{m}$. To pull high resistance electrodes, we extensively studied the P-97 operation manual (2009, Rev.2.03) and the P-1000 \& P-97 pipette cookbook (2011, Rev.G) provided by the Sutter Instruments company. Further, Nicola Strenzke gave us some important tips about pulling sharp electrodes. On the one hand, we optimized the program by fine-tuning the five main parameter of the puller (Heat, Pull, Velocity, Time and Pressure). Here, the heat and the strength of the pull were the important variables. Further, we also had to optimize the distance of the glass capillaries to the heat filament as well as the heat filament to the air jet. In addition, we changed the filament itself; the trough filament (Sutter Instruments, FT330B) provided higher resistance than the classical box filament. A further critical variable was the inner and outer diameter of the glass capillaries. For example, glasses normally used for patch pipettes with an outer diameter (O.D.) of $1.5 \mathrm{~mm}$ and inner diameter (I.D.) of $1 \mathrm{~mm}$ only lead to resistance of $\sim 20-50 \mathrm{M} \Omega$ and did not allow successful impaling of the cell bodies. However, glasses with smaller inner and outer diameters, for example of 1-1.2mm O.D. and 0.5-0.6mm I.D. lead to the desired high resistance electrodes. At the end, we used borosilicate glass with filament with an 
O.D. of 1.2mm and I.D. of 0.6mm (Sutter Instruments, BF120-60-10) and had final resistance of around 100-300 $\mathrm{M} \Omega$ (measured with $3 \mathrm{M} \mathrm{KCl}$ ). For the experiment, we tip-filled our sharp microelectrodes with $4 \%$ Neurobiotin, dissolved in $0.1 \mathrm{M}$ Tris buffer, and backfilled the electrode with $3 \mathrm{M} \mathrm{KCl}$.

The sharp electrodes were positioned above the outer segments of the photoreceptors with the help of a 60x objective (Fig.3.1B) and slowly inserted into the retina (1 $\mu \mathrm{m}$ steps, diagonal axis). The depth of the electrode was monitored through the $60 \mathrm{x}$ objective as well as through the remote control (keypad SM5, Luigs \& Neumann). The thickness of the salamander retina is $\sim 200-300 \mu \mathrm{m}$, we expected the bipolar cell bodies roughly after 70-120 $\mu \mathrm{m}$ (outer segments + photoreceptor cell bodies + outer synaptic layer) (Roth, 1987). To impale cells, we applied small electric pulses $\left(15 \mathrm{~V}_{\mathrm{p}-\mathrm{p}} 10 \mathrm{kHz}\right.$ square wave pulses, Buzz button in the I-Clamp pane). Membrane potentials were recorded using a MultiClamp 700B amplifier (Molecular Devices, San Jose, CA) in current-clamp mode and digitized at 20kHz using a Digidata 1440A (Molecular Devices) and stored on a computer with pClamp 10 software (Molecular Devices). After the experiment, the data were extracted and downsampled to a $1 \mathrm{~ms}$ resolution with a running median (code was adapted from Michael Weick written in MATLAB, The Mathworks, Natick, MA).

\subsubsection{Multielectrode array}

To record simultaneously from bipolar and ganglion cells (goal 4), the retinal ganglion cells spiking activity was recorded extracellularly with a perforated multielectrode array (pMEA). The perforated MEAs contain small holes between the electrodes, which allow slight suction from underneath the array controlled by a suction pump outside the setup (Fig.3.1A). We used the 60pMEA100/30iR (MultiChannel Systems, Reutlingen, Germany), where 60 Titanium nitride electrodes with a diameter size of $30 \mu \mathrm{m}$ and electrode distance of $100 \mu \mathrm{m}$ are embedded in a perforated polyimide foil. An advantage of the perforated MEA is that the suction from below leads to a better oxygen and nutrient supply and a better signal-to-noise ratio due to improved contacts between the electrodes and the retina (Reinhard et al., 2014). Further, the retina can be kept in place without the need of an additional filter membrane that presses the retina onto the electrodes. Thus, the perforated system is ideal for us and provides free access to insert sharp electrodes into the retina. The perforated multielectrode array is also the main difference to the previous studies that combined MEA and single cell recordings (e.g. Asari and Meister (2012)). Further, we used a 60x objective to see the electrodes of the MEA (see Fig.3.1B) and place the sharp pipette exactly above the electrodes of the MEA that showed high ganglion cell spiking activity. This design allowed us to maximize chances of recording pairs of bipolar and ganglion 
cells, which was needed for the study of subunits in ganglion cells by our group (Liu et al., 2017). The extracellular recorded voltage traces were amplified and band-pass filtered $(300-5000 \mathrm{~Hz})$, digitalized at $25 \mathrm{kHz}$ and stored with the data acquisition software MC Rack (MultiChannel Systems).

\section{Spike Sorting}

Multielectrode array recordings are powerful techniques to record the activity of many neurons simultaneously. From the 60 electrode sites, we usually recorded 200-300 retinal ganglion cells. Thus, one electrode typically records spikes from several cells. Further, the signal of an individual cell can also be recorded by several electrodes. To assign the different spike waveform to individual ganglion cells, we apply an analytical technique named spike sorting. Spike sorting tries to group spikes into clusters based on the similarity of their waveforms and the goal is that each cluster represents a separate cell (for a review see Lewicki (1998)). Each neuron produces a unique spike waveform that mainly depends on the distance and orientation of the cell towards the recording electrode (Gold et al., 2006).

We used a custom-made spike sorting program developed by Ofer Mazor (Harvard University, Cambridge, USA) in Igor Pro 6.37 64-bit (WaveMetrics, Lake Oswego, USA) (Pouzat et al., 2002). The program is semi-automatic. First, electrodes with similar voltage traces are grouped (measured through cross-correlations between the voltage traces). Then, the spikes are detected when passing a threshold (4 standard deviation above the noise level) (Quiroga et al., 2004). The spike-waveforms are aligned by the peaks, which was set at the $15^{\text {th }}$ sample point and cut to a duration of $1.8 \mathrm{~ms}$ (45 sampling points). The spike waveforms of each electrode group are clustered with a Gaussian mixture model. The resulting clusters were manually inspected and improved. For example, if the refractory period was violated, the waveforms were split into two or more clusters (splitting); when two clusters strongly overlapped, their waveforms were merged into one cluster (joining). Each cluster was graded according to the amplitude of the waveform, refractory period (\% interspike-intervals below $3 \mathrm{~ms})$ and the distance between clusters.

\subsubsection{Light stimulation}

Light was projected onto the photoreceptor layer through a $4 \mathrm{x}$ objective of an upright microscope (see Fig.3.1B). The light source was a monochromatic white OLED monitor (eMagin, $800 \times 600$ pixels, 60 or $75 \mathrm{~Hz}$ refresh rate). To de-magnify the OLED image and to include the image into the light path of the microscope, the light was first projected through two lenses and a beamsplitter (Fig. 3.1B). The design helped us to have a sharp image of the OLED 
monitor at the focus location of the microscope. Thus, to ensure a sharp image at the photoreceptor layer, we focused on the outer segment with a $4 \mathrm{x}$ objective. To additionally finetune the focus in the range of micrometers (due to different focus of the camera), we played stimuli with fine spatial structures and focused the monitor at the position which maximized the spiking rate or membrane potential of the recorded cells.

The total size of the stimulation area of the OLED (800x600) was $2 \times 1.5 \mathrm{~mm}$, which was larger than the electrode area of $0.93 \mathrm{~mm} \times 0.53 \mathrm{~mm}$. Each pixel had a size of $2.5 \times 2.5 \mu \mathrm{m}$, which allowed us to project light stimuli on a photoreceptor resolution. The mean light level (perceived as gray) was about $2.5 \mathrm{~mW} / \mathrm{m}^{2}$, which correspond approximately to a low photopic range with $\sim 1.27 \times 10^{4}$ isomerization per rod per second (for example indoor light). At this light level, the rods are considered to be saturated (Farrow et al., 2013) or they are considered to be the least active (Tikidji-Hamburyan et al., 2017). Further, we calibrated and gamma-corrected the OLED, to ensure a linear light signal. The light stimuli were programmed and presented by a custommade software written in $\mathrm{C}++$ and OpenGL libraries. The light stimulation, multielectrode array and single-cell recording stage were inside a Faraday cage and covered by a black curtain during the experiment. Thus, the retina was only stimulated by the light stimuli presented on the OLED.

\subsection{Identifying recorded cell types}

For the multielectrode array recordings, it is commonly accepted that the recorded spikes come from retinal ganglion cells. The retinal ganglion cell layer on the one hand directly faces the electrodes. On the other hand, only high amplitude voltage changes can be measured with extracellular electrodes and the ganglion cells are the main cell type in the retina that generates such action potentials.

The identification of the recorded cell types with the sharp microelectrodes was more challenging. We used staining and imaging techniques to identify the morphology of the recorded cells. Yet, in some experiments, the staining failed and thus we used other criteria to distinguish bipolar cells from photoreceptors and amacrine cells. In this section, we are going to review the techniques individually.

\section{Staining}

To assess the morphology of the recorded single cells, we tip-filled our glass electrodes with 4\% Neurobiotin (Vector Laboratories, Burlingame, CA) dissolved in $0.1 \mathrm{M}$ Tris buffer. At the end of an intracellular recording, we injected the Neurobiotin into the cell with positive and 
negative current pulses. We developed the current injection protocol with the help from Lauw Klaassen from the group of Maarten Kamermans who works with sharp microelectrodes and Neurobiotin in the retina. The amount of positive and negative current injection was celldependent. For positive current, we depolarized the cell to approximately $0 \mathrm{mV}$ (we tried to avoid going positive). For negative pulses, we hyperpolarized the cell to approximately -80 to $-90 \mathrm{mV}$ (we tried to avoid to go more negative than $-90 \mathrm{mV}$ ). We used two types of protocol for Neurobiotin injection: block design and alternating design. In the block design, we injected first positive current pulses (0.5sec current, $2 \mathrm{sec}$ break) for $\sim 2-4$ minutes, let the cell rest for 0.5-1 minute and then injected negative current pulses (0.5sec current, $2 \mathrm{sec}$ break) for $\sim 2-4$ minutes. In the alternating design, we injected first for 2 minutes positive pulses ( $0.5 \mathrm{sec}$ current, $1 \mathrm{sec}$ break), let the cell rest for $\sim 0.5-1$ minute and then injected alternating positive and negative pulses (4 positive pulses, 2 seconds break, 2 negative pulses) for 2-4 Minutes. Our experience was that the pattern of pulses was not critical for the staining. Important was to have at the beginning positive pulses for at least 2 minutes to fill the cell body with Neurobiotin. The negative pulses helped to spread the Neurobiotin along the dendrites (personal communication with Lauw Klaassen). There was a tendency that we had more neighboring neurons filled the longer we applied the current injection. Currently, we mainly use the alternating design for a total of $\sim 5$ minutes. Note, before using Neurobiotin, we tried to fill the cells with Lucifer Yellow. Yet, we were not successful in staining the cells; further, the glass pipettes commonly were clogged. With Neurobiotin we could overcome these problems.

At the end of an experiment, the retina was fixated and further processed to image the cells with a confocal microscope. We used two different staining protocols. The first protocol was introduced with the help of the group of Jochen Staiger where Patricia Sprych taught me the procedure. Here, we fixated the retina in a $4 \%$ paraformaldehyde solution containing $15 \%$ (vol/vol) picric acid in 0.2 M phosphate buffer (PB), for $\sim 24$ hours. The fixation was then washed out extensively with PB ( $\sim 15$ times for 15 minutes at room temperature), followed by one rinse in Tris Buffer and one rinse in Tris buffer saline (TBS). Further, the retinas were washed two times with TBS containing 0.5\% Triton-X 100 (TBST) (15min each) and kept overnight in TBST containing Alexa Fluor 488 Streptavidin 1:300 (Thermo Fisher Scientific, MA, USA) at $4^{\circ} \mathrm{C}$ on a rocking plate. Then, the retinas were rinsed two times with TBST and one time with TBS, before a nucleus staining was performed with TO-PRO-3, 1:1000 (Thermo Fisher) in TBS for 5 minutes (at room temperature). The retinas were washed in TBS and TB buffer and mounted and coverslipped with Aqua-Polymount.

In the second protocol, we fixated the retina in $4 \%$ formaldehyde in Phosphate-buffered saline (PBS) for 1-2 hours and left it overnight in PBS. We washed the retina five times with PBS 
(15 minutes at room temperature) and rinsed it two times with $0.5 \%$ Triton-X 100 in PBS. The retina was then kept overnight in the PBS buffer containing Triton and Alexa Fluor 488

Streptavidin 1:300 at $4^{\circ} \mathrm{C}$. Before we performed a staining of the nuclei with TO-PRO-3 in PBS (1:1000), we washed the retina two times in the PBS buffer containing Triton and one time in PBS (all at room temperature). Then, we washed the retina three times in PBS and mounted and coverslipped the retinas with Aqua-Polymount. The second protocol was made with the help of Michael Weick.

With both protocols, we could get very beautiful stainings and no clear effect of the protocols on the quality of the stained cell could be observed.

We then inspected the retinas with fluorescence microscopy to see whether and where we have successfully stained neurons and then we went to the confocal microscopy to acquire a zstack. We analyzed the images with MATLAB and did two-dimensional representations of the recorded cells by maximum projections in the $x-z, y-z$ and $x-y$ plane. We identified the recorded cell types in the following way. Photoreceptors were identified by their clear outer segments and their cell bodies in the outer layer of the retina (see Fig.3.2). Bipolar cells were identified by their bipolar shape; they made contacts with the outer (photoreceptors) and inner (amacrine and ganglion cells) parts of the retina and usually their cell bodies lay just below the outer synaptic layer (see Fig.3.2). The amacrine cells only make contacts in the inner retina but not with photoreceptors in the outer retina and their cell bodies lay closer to the ganglion cells. Further, we almost exclusively recorded from amacrine cells that possessed long neurites that stratified in the same layer as their cell bodies (see Fig.3.2).

When the staining and imaging of the cell was successful, we used the morphology of the cell to identify the recorded cell type. Yet, the imaging did not always work, sometimes, the cell was lost before the dye injection, sometimes, the retina could not be removed from the perforated multielectrode array and sometimes no cells were stained in the tissue. Thus, we developed additional criteria to distinguish cell types. 

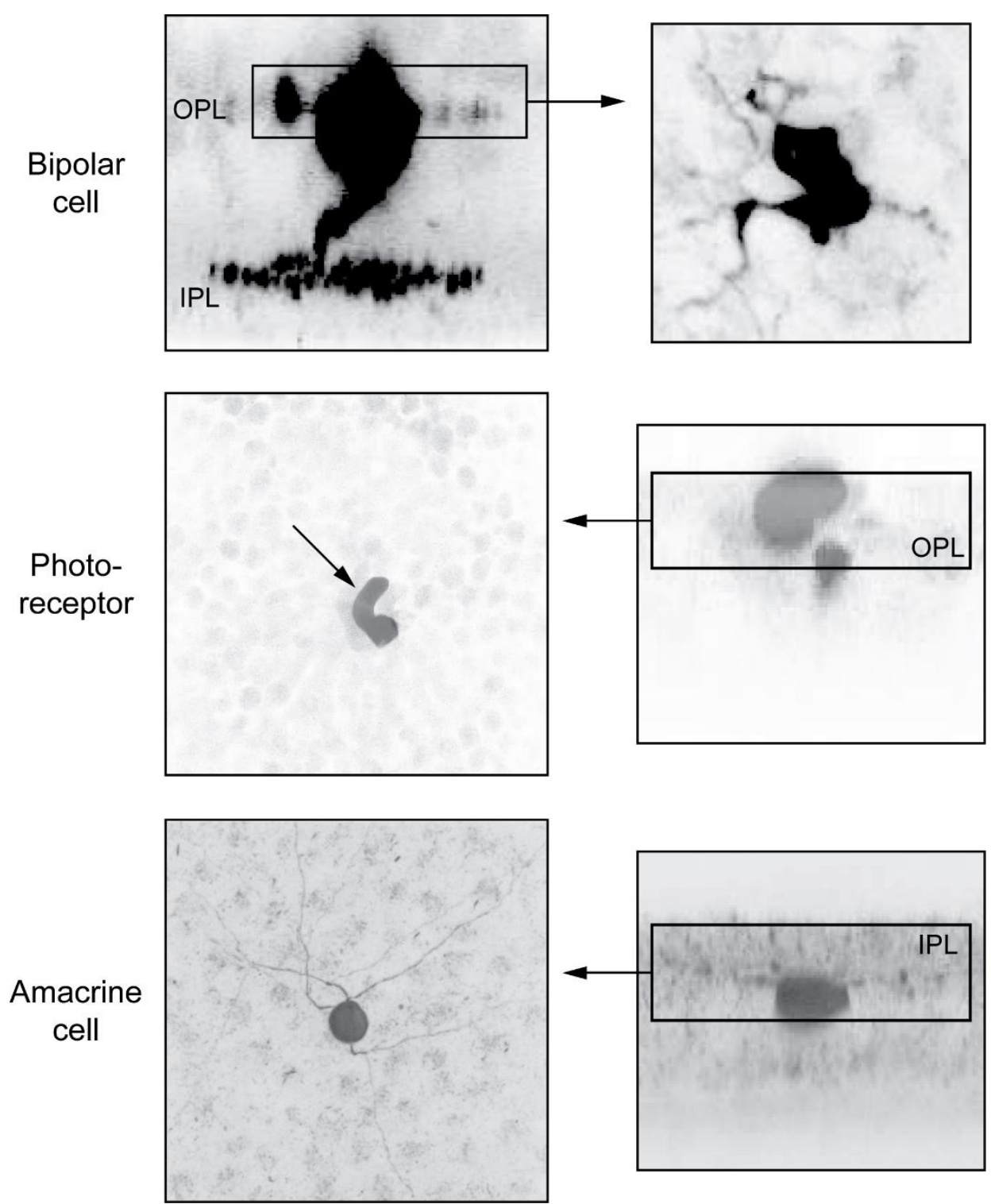

Figure 3.2. Identifying cell types with morphology. The first cell is a bipolar cell, which was identified based on the connection in both the outer plexiform layer (OPL) and the inner plexiform layer (IPL). The inset shows the dendrites at the outer plexiform layer. The second cell is a photoreceptor, which was identified based on the outer segment (see arrow). Further, the photoreceptor made contacts only into the outer plexiform layer (see rectangle). The third cell is an amacrine cell with neurites stratify only into the inner plexiform layer (see rectangle).

\section{Distinguishing bipolar from photoreceptor cells}

Photoreceptors show OFF responses to light and their traces have some similarity to some OFF bipolar cells. Thus, to distinguish bipolar cells from photoreceptors, we used in some recordings, the AMPA/kainate antagonist CNQX to check whether the light responses of our recorded cell is abolished. CNQX blocks the excitatory inputs to the OFF bipolar cells while the photoreceptors are not affected. Thus, if our recorded cell continued to respond after the application of CNQX, we knew that the cell was a photoreceptor. 
Bipolar cells were further distinguished from photoreceptors based on the recording depth. We expected the bipolar cell bodies approximately after 70-120 $\mu \mathrm{m}$ (outer segments + photoreceptor cell bodies + outer synaptic layer) (Roth, 1987). Thus, the recorded responses

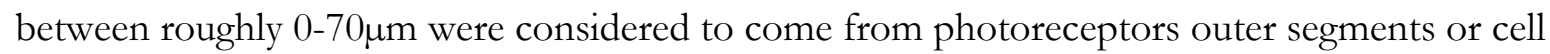
bodies. Additionally, those cells that were morphologically identified as photoreceptors, showed smaller receptive field sizes, while bipolar cell receptive fields were bigger and on average around $110 \mu \mathrm{m}$ (2sigma). Further, all recorded photoreceptors showed stereotypical response traces to light increments and decrements, usually starting with a slow increase to light decrement and a fast hyperpolarization to light increments. However, for few bipolar cells we observed similar response traces for light increment and decrements.

\section{Distinguishing bipolar from amacrine cells}

Bipolar cells were distinguished from amacrine cells by the sign of retinal ganglion cell responses to current injection. We injected positive and negative current pulses into our recorded cells $(50-500 \mathrm{pA}, 500 \mathrm{~ms}$ duration, $2 \mathrm{sec}$ break). If the ganglion cells increased their firing rate to the positive current injection, we identified the recorded cell as a bipolar cell (similar to Asari and Meister (2012)). If on the other hand, the ganglion cells increased the firing rate to a negative current injection, we identified the recorded cell as an amacrine cell (similar to de Vries et al. (2011)). The idea is that bipolar cells increase their excitatory neurotransmitter release under a depolarization and therefore activate the connected ganglion cells. The amacrine cells on the other hand, decrease their inhibitory neurotransmitter release under a hyperpolarization and therefore increase the responses of the connected ganglion cells. In Figure 3.3, we show the location of a simultaneous recorded bipolar and ganglion cell and the corresponding positive responses of the ganglion cell to current injection.

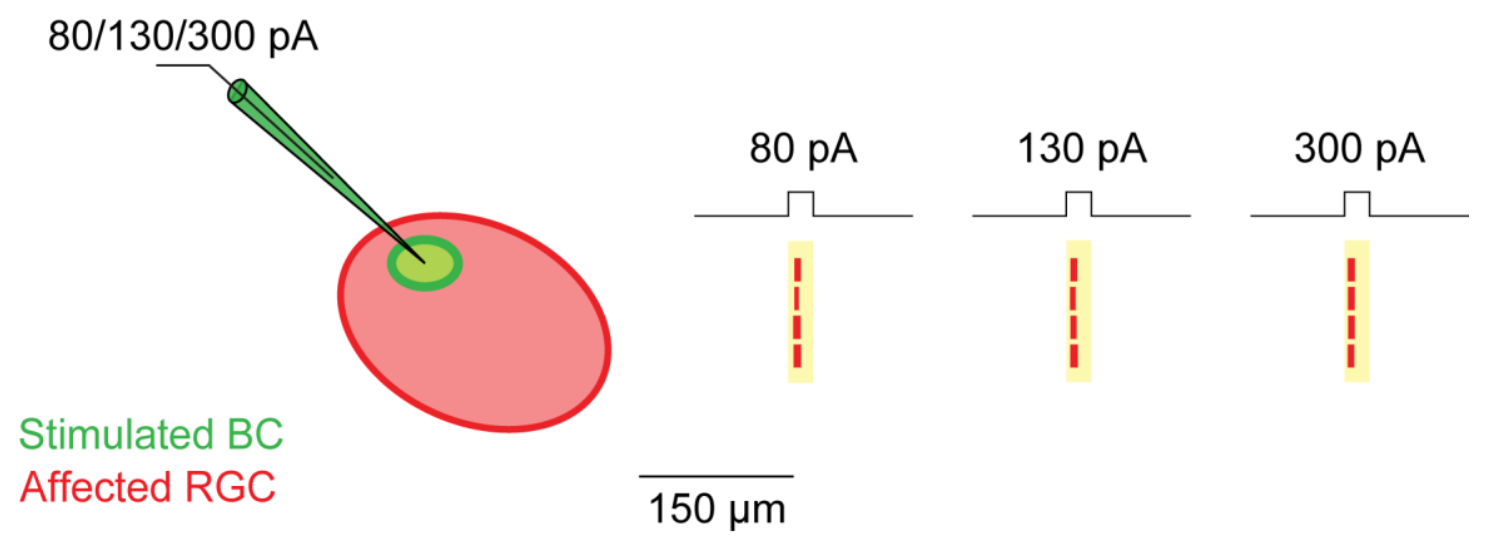

Figure 3.3. Retinal ganglion cell responses to current injection into a bipolar cell. Simultaneous recording of a bipolar (BC) and a retinal ganglion cell (RGC). Circles show the corresponding receptive field location and size. 
On the right, the responses of the retinal ganglion cell to different amount of current injection. The lines are spikes.

Amacrine cells were further distinguished from bipolar cells based on the spiking activity. We often observed that the amacrine cells identified with morphology showed spiking activity (fast transient events) with amplitudes of around $20-50 \mathrm{mV}$. Further, the recording depth gave us an orientation when approximately the amacrine cell bodies started. For example, cells that we recorded directly after the photoreceptor layer were considered as bipolar cells.

\subsection{General response characterization}

As we have seen, bipolar cells are separated into different types based on their response properties (e.g. sustained and transient). Thus, in our goal 1 we characterized bipolar cells based on their responses to different light stimuli. We investigated three main response characteristics: response polarity, receptive field and temporal properties. In this chapter, we describe the analysis performed for each main characteristic separately. To characterize the general response properties we used three types of light stimuli (Fig.3.4):

1. We used a periodic full-field light step stimulus, where in one period, the whole screen changed successively from gray $(1 \mathrm{sec})$ to white $(1 \mathrm{sec})$ to gray $(1 \mathrm{sec})$ to black $(1 \mathrm{sec})$ (see Fig.3.4A). For some cells, the full-field light step stimulus changed from black $(1 \mathrm{sec})$ to white $(1 \mathrm{sec})$, without a gray intermediate screen (see Fig.3.4B). We presented the full-field light steps for around 1 minute ( $\sim 15$ repetitions).

2. We used a spot stimulus, where a spot was presented on a gray background $(0.5 \mathrm{sec})$, followed by a gray screen $(1 \mathrm{sec})$ (see Fig.3.4C). The spot diameter changed randomly

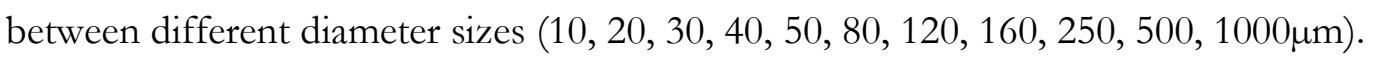
Each spot diameter was shown $\sim 10$ times. First, we showed the stimulus only with black spots ( $\sim 3$ minutes) then the white spots ( $\sim 3$ minutes). For some cells, we also recorded a spot stimulus that directly changed between black $(0.5 \mathrm{sec})$ and white

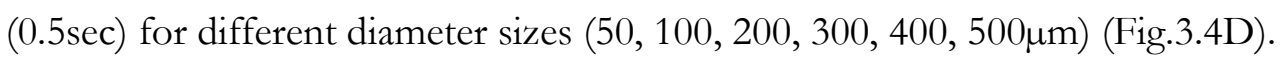

3. We used white noise stimuli, either full-field Gaussian white noise (Fig.3.4E) or binary white noise (Fig.3.4F). The full-field Gaussian white noise stimulus is a uniform contrast flicker where the intensity (contrast) of the full screen changes every $33 \mathrm{~ms}$ to a value randomly drawn from a Gaussian distribution. One standard deviation of the 
full-field stimulus corresponded to $30 \%$ contrast (from the mean light level at gray). We recorded the full-field stimulus usually for 10 minutes.

For the binary white noise stimulus, the screen was subdivided into a checkerboard layout with a pixel size of $30 \times 30 \mu \mathrm{m}$ (see Fig.3.4F). In some experiments, we also used smaller pixel sizes of $10 \times 10 \mu \mathrm{m}$ or $22.5 \times 22.5 \mu \mathrm{m}$. For the binary white noise stimulus, the pixels randomly and independently changed between black and white with equal probability, thus, the intensity values of the pixels were uncorrelated to each other. For most cells, the intensity of the pixels changed every $33 \mathrm{~ms}(60 \mathrm{~Hz})$ or $40 \mathrm{~ms}(75 \mathrm{~Hz})$. The speed of the stimulus was reduced to 66 or $99 \mathrm{~ms}$ $(60 \mathrm{~Hz})$, if the cell did not respond to a fast change of $33 \mathrm{~ms}$. Dependent on the research question, we recorded the binary white noise stimulus between 15-60 minutes.

The response polarities, as well as the temporal properties, were investigated with all three stimulus types. The receptive field size was investigated with the spot stimulus and the binary white noise stimulus. All analysis were programmed with MATLAB (The Mathworks, Natick, MA). 
A

light steps

gray preframes

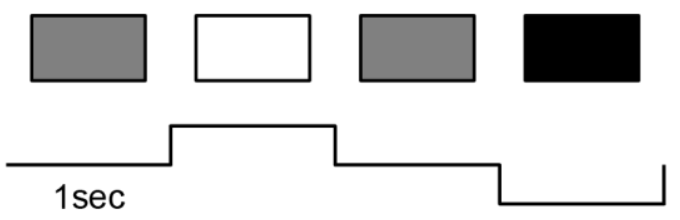

C

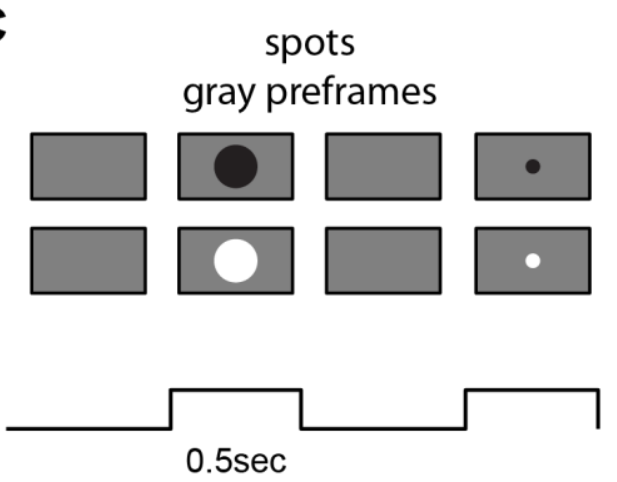

E

gaussian white noise
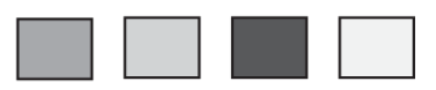

$33 \mathrm{~ms}$
B

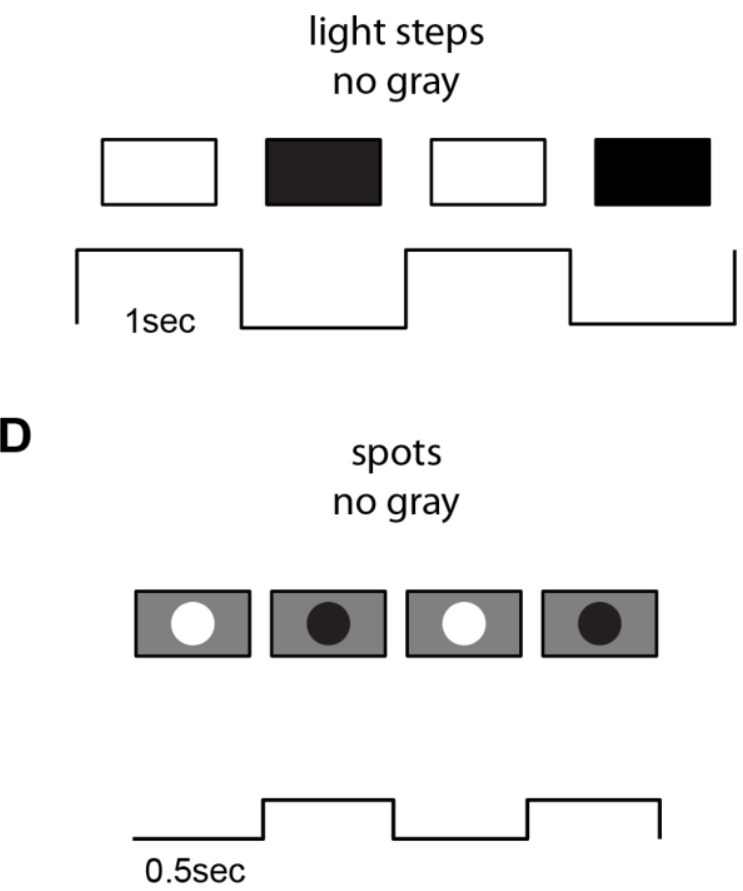

$\mathbf{F}$ binary white noise

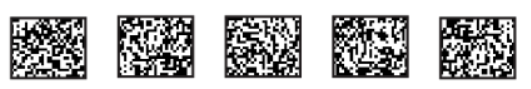

$33 \mathrm{~ms}$

Figure 3.4. Light stimuli used for the general response characterization. A. Full-field light steps with gray preframes (step duration 1 second). B. Full-field light steps without gray preframes. C. Spot stimulus, where a spot was presented on a gray background $(0.5 \mathrm{sec})$, followed by a gray screen $(1 \mathrm{sec})$. The spot diameter changed randomly between different diameter sizes. D. Spot stimulus with different diameter sizes without gray preframes. E. Full-field Gaussian white noise stimulus. F. Binary white noise stimulus, where the screen is subdivided into small pixels that randomly change between black and white.

\subsubsection{Response polarity}

We characterized whether the bipolar cells respond to light increments (ON cells) or light decrements (OFF cells) by analyzing their responses to the full-field light steps (Fig.3.4A-B). Further, we compared whether the response polarity changed under the spot stimulus and the white noise stimulation. 


\section{Full-field light steps}

For each trial, we defined the baseline level of the cell by building the average response over the last $200 \mathrm{~ms}$ of the first gray screen (see red dashed rectangle in Fig.3.5). Then, we assessed the depolarization and hyperpolarization, by subtracting for each trial the baseline level from the response and built the average over the baseline-corrected single trial responses. A depolarization is defined as a response that is more positive compared to the baseline membrane potential at gray, a hyperpolarization is a response that is more negative compared to the baseline level at gray (see Fig.3.5).

From the average trace, we computed a polarity index that took values close to 1 for ON cells and values close to -1 for OFF cells (Franke et al., 2017). The response polarity of a cell was defined as the difference between the response (r) during the white $(\mathrm{ON})$ and black (OFF) light step, divided by the total response during these two light steps:

$$
\text { Polarity }=\frac{\sum_{t=1}^{f} r O N(t)-\sum_{t=1}^{f} \operatorname{rOFF}(t)}{a b s\left(\sum_{t=1}^{f} \operatorname{rON}(t)\right)+a b s\left(\sum_{t=1}^{f} \operatorname{rOFF}(t)\right)}
$$

where $f=1000 \mathrm{~ms}$, which is the total duration of one light step.
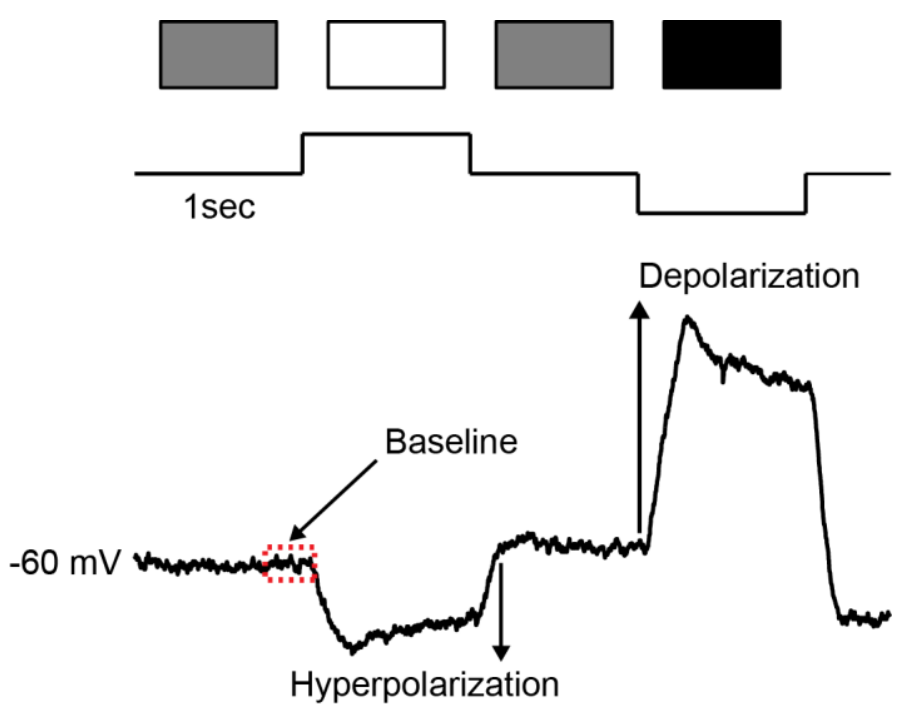

Figure 3.5. Response polarity characterized with full-field light steps.

Note, for the cells where we recorded a black-white light step without an intermediate gray screen, the baseline response was built from the response to a gray screen (last 200ms) that was presented before the starting of the light step. 
Spots

For the spot stimulus, the trials were baselined in the same way, by subtracting from each trial, the averaged activity over the last $200 \mathrm{~ms}$ of the gray screen. For each spot diameter size, the average over the baseline-corrected trials was built. For the optimal spot size (see section 3.5.2), the polarity index was computed in the same way as described for the full-field light steps ( $f=500 \mathrm{~ms})$. For the cells that were recorded with a black-white switching spot (no gray), the baseline was built by the gray screen that was presented at the beginning (200ms before the spot).

\section{White noise}

From the full-field white noise as well as the binary white noise stimulus, we quantified the response polarity by building the temporal filter of the cell, also referred to as voltagetriggered-average. The exact calculation of the temporal filter will be introduced in the next subchapters. In Figure 3.6, two temporal filters of bipolar cells are pictured. The response polarity is reflected in the sign of the peak of the filter. A positive peak indicates that the cell prefers light increments and is therefore an $\mathrm{ON}$ cell (Fig.3.6A), a negative peak represents an OFF cell (Fig.3.6B).

A

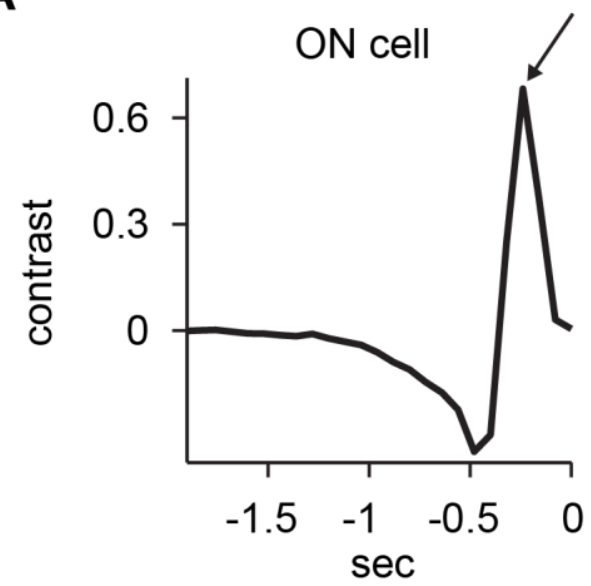

B

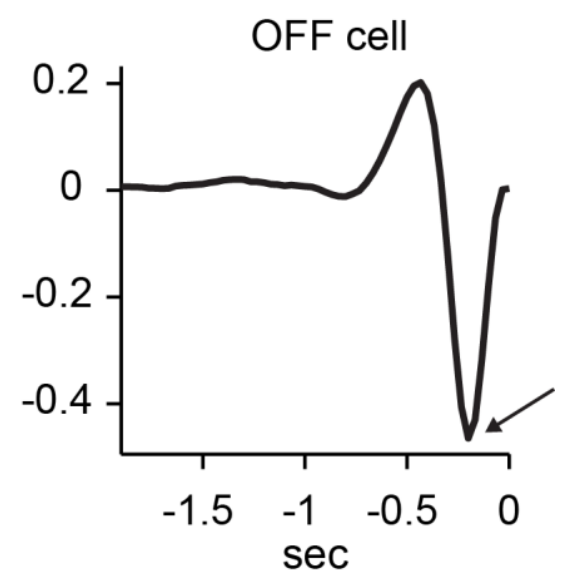

Figure 3.6. Response polarity characterized with white noise. A. Example ON cell that shows a positive peak just before 0 . B. Example OFF cell that shows a negative peak just before zero. Time zero is the membrane potential change.

\subsubsection{Receptive field center and surround estimation}

We estimated and compared the receptive field center with both classical spot stimuli and binary white noise. 


\section{Center and surround estimation with spots}

The estimation of the center size with spots is done in two steps. First, the position of the receptive field center is determined by moving a flashing spot over the retina and searching for the location that maximally activates the recorded cell (Fig.3.7A). Second, the receptive field size is estimated with varying spots sizes presented over the determined center position (Fig.3.7B). For each diameter size, the maximum of the average baselined response is represented in a tuning curve (Fig.3.7C). The receptive field center size is approximated by the diameter that induces the optimal response (see arrow in Fig.3.7C). The optimal response is defined as the diameter size where a further increase in spot diameter did not increase the response amplitude by more than 20\% (see Van den Bergh et al. (2010) for a similar logic). The 20\% threshold was introduced to avoid high center diameter sizes for cells with a plateau-like region around the maximum.

A

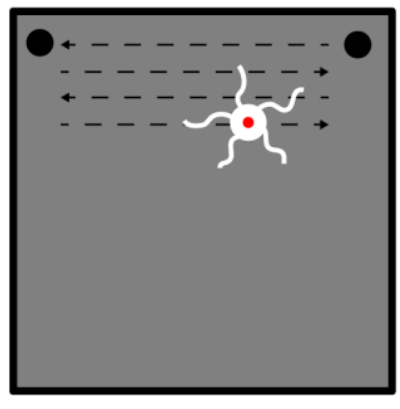

B

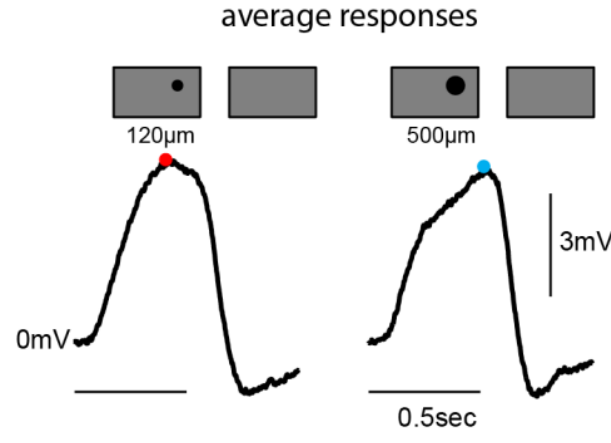

C tuning curve

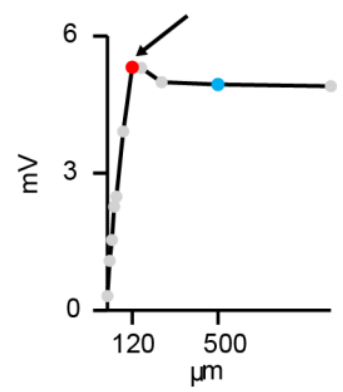

Figure 3.7. Receptive field center estimated with spots. A. We manually determined the location of the cell by moving a flashing spot over the retina. Once we found the location (red dot), we showed spots with different diameters. B. Average baselined response traces of an OFF bipolar cell to spots with different diameters. C. Tuning curve, showing the maximal response amplitudes for each spot size for the example cell in $\mathrm{B}$. The arrow points to the estimated receptive field size.

The surround inhibition was measured by comparing the response of the receptive field center to the response to a large spot i.e. whose diameter exceeds the receptive field center (Fahey and Burkhardt, 2003; Hare and Owen, 1990; Krieger et al., 2017). If the response to the large spot, compared to the center spot, is reduced, the bipolar cell shows an inhibitory surround. The large spot was defined as the spot diameter (up to $500 \mu \mathrm{m}$ ) with the smallest response after the spot inducing the maximum response (see corresponding blue point in Fig.3.7C). We chose the spot diameter with the smallest response, instead of the largest spot diameter, to avoid underestimation of the surround for cells that show a sudden increase to the largest spot diameter. The increased response to the biggest spot could be an effect of a far surround, which 
is different from the classical antagonistic surround that we were investigating. The index of the surround was defined as follows:

$$
\text { surround strength }=1-\left(\frac{\max \left(r_{\text {largespot }}\right)}{\max \left(r_{\text {centerspot }}\right)}\right)
$$

A value close to 0 stands for no inhibitory surround. A value close to 1 stands for a strong surround. Note, for the spot that switched between black and white without gray, the same logic was applied. Further, to compare the surround index between the two types of spot stimuli, only the responses up to $500 \mu \mathrm{m}$ were taken into consideration.

\section{Center estimation with binary white noise}

To remove slow fluctuations in the membrane potential, the responses to the binary white noise were first de-trended with a high-pass filter (fourth-order Butterworth filter, $0.1 \mathrm{~Hz}$ ). The slow fluctuations as pictured in Figure 3.8 were observed for some cells during long recordings. They were removed to avoid over- or underestimating the response.
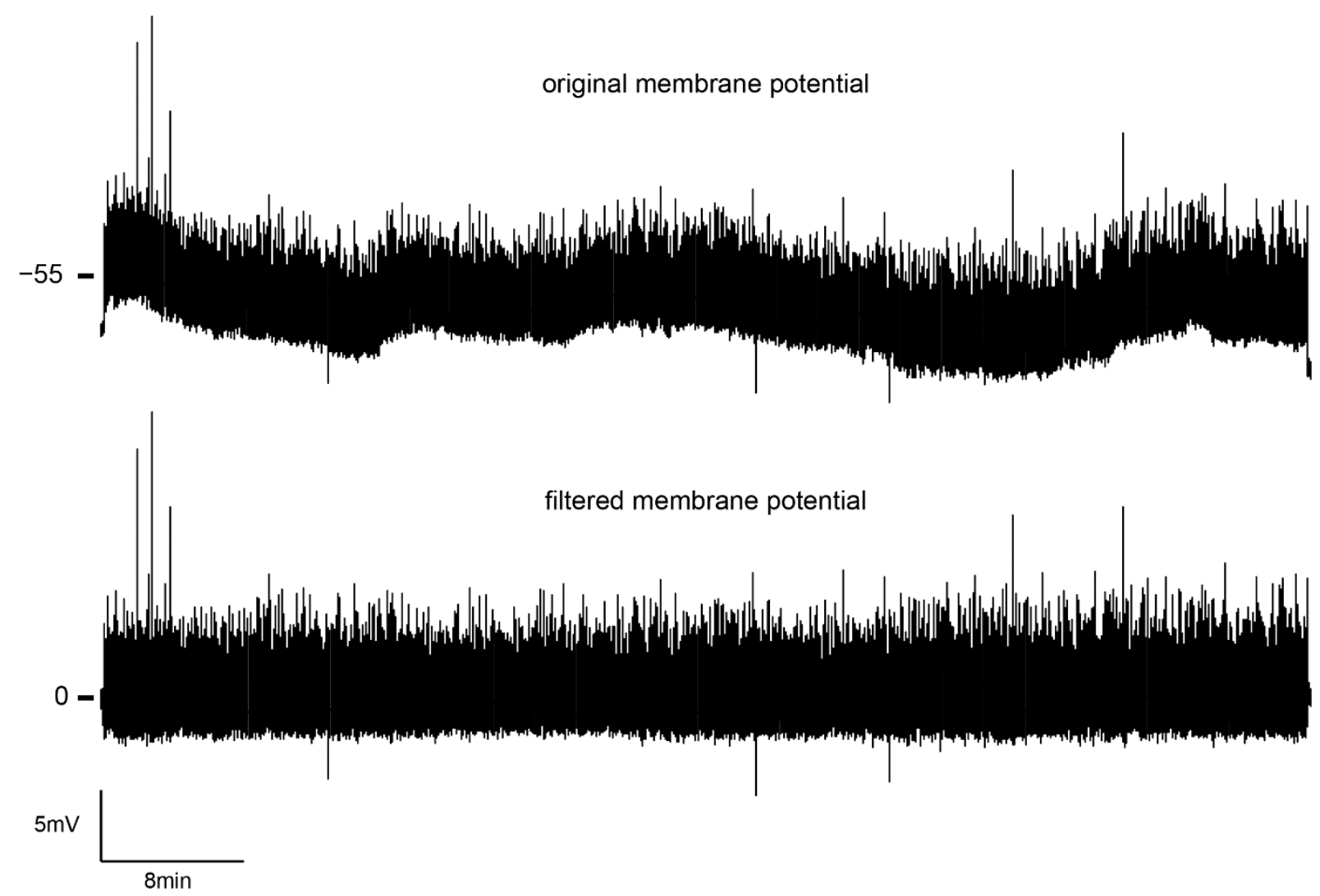

Figure 3.8. Removing slow fluctuations from the membrane potential traces. 
To estimate the receptive field size with binary white noise, the voltage-triggered-average was built. To do so, the recorded membrane potential to the binary white noise stimulus is first averaged per frame and subtracted from the overall mean (mean over the whole recording period, see Fig.3.9). The stimulus intensity values up to 2 seconds preceding each frame were collected (see red, blue and green rectangles in Fig. 3.9) and weighted by the corresponding membrane potential deviation for that frame. Finally, the average over the weighted stimulus sequence was built, which provides the spatiotemporal filter of the cell (Fig.3.9). The spatiotemporal filter or also voltage-triggered average shows which stimulus on average elicits a membrane potential change in the bipolar cell (for the analog with spikes, the spike-triggered-average see Chichilnisky (2001)). Through the averaging, the pixels that lead to the membrane potential change pop out, while for the other pixels the random contrast averages to gray.

The spatiotemporal filter contains information about the spatial location of the receptive field of the cell and the change of the response over time. For example, when looking at the frame with the maximum response in Figure 3.9 (red frame), we see the location and size of the receptive field of the recorded bipolar cell. Further, we see that over time, the pixels inside the receptive field location change, for example, first they are gray then black then gray.

To assess the spatial and temporal components of the spatiotemporal filter in a standardized way, we used the singular-value-decomposition (SVD, MATLAB command svd) (Gauthier et al., 2009; Liu et al., 2017). The SVD decomposes the spatiotemporal filter into two orthogonal matrices of space and time. Then, we used the highest ranked spatial and temporal components as an approximation of the spatial and temporal receptive field (Fig. 3.9). To estimate the receptive field size, we fitted a two-dimensional Gaussian function to the spatial component. As an approximation of the receptive field, we chose a contour of 2 standard deviation of the fitted Gaussian (red ellipse, Fig. 3.9). From here, we determined the diameter = $\sqrt{a \bullet b}$, where $\mathrm{a}$ is the major and $\mathrm{b}$ the minor axis of the ellipse contour. 
binary white noise

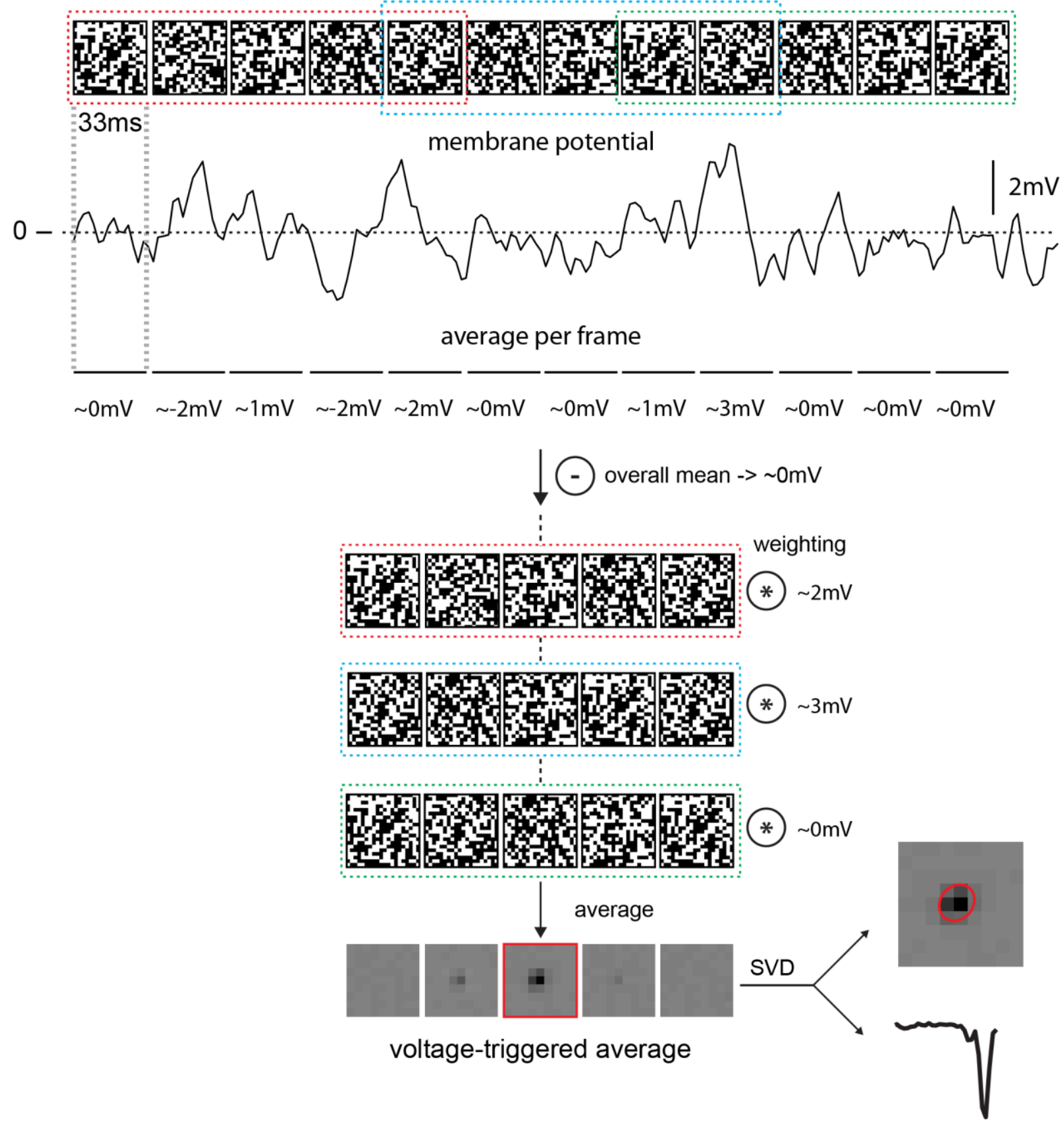

Figure 3.9. Receptive field center estimation with white noise. The recorded membrane potential is first averaged per stimulus frame (33ms) and the deviation to the overall mean is computed. Then, the stimulus intensity values up to 2 seconds preceding each frame were collected (red, blue and green dashed rectangles) and weighted by the corresponding membrane potential deviation for that frame. Finally, the average over the weighted stimulus sequence was built to assess the voltage-triggered average. From here, the spatial and temporal components were deduced by the method of singular value decomposition (SVD). 


\subsubsection{Temporal properties}

We estimated and compared the temporal properties with the full-field light steps, the spots and the white noise stimuli (see again Fig.3.4 for the stimulus design). We focused on the latency (fast/slow), the duration (sustained/transient) and the oscillation frequency of the response.

\section{Full-field light steps}

Response onset. To compute how fast or slow a cell responded to the stimulus, we defined the time from the onset of the preferred light step (OFF or ON) to the onset of the response of the cell in ms (yellow dot in Fig.3.10). The response onset ( $t_{\text {onset }}$ ) was calculated as the first point that exceeds 4 standard-deviations of the gray baseline activity:

$$
\text { Response onset }=\min \left(r(t)>4 \sigma_{\text {baseline }}\right)
$$

The latency measurement is similar to a current study of bipolar cell glutamate release (Franke et al., 2017). In other studies, different measures are used to quantify the response latency. For example, the time it takes for a cell to reach the peak (maximum) (green dot in Fig.3.10) or the maximal derivative (slope) of the response trace (pink star in Fig.3.10, see e.g. Baden et al. (2013a)). We did not use the peak as a latency measure; because for some of our cells, the response continuously increased during the full light step period (see Fig. 3.10B). If we would have taken the peak, these cells would have had a response onset of $1000 \mathrm{~ms}$, yet clearly, they responded much earlier. Further, we did not take the maximal slope, because for cells with similar response profiles, the maximal slope could be close to the peak (Fig.3.10C) or at the rise of the response (Fig.3.10A).

Sustained-transient-Index (STi). The sustained vs. transient responses were distinguished by computing the ratio between the mean response level at the end (steady state) and the peak of the response. The peak response was defined as the maximum response to the preferred light step (green point in Fig.3.10). The steady state was computed by taking the mean of the response (r) over half the interval from the peak ( $\left.t_{\text {peak }}\right)$ to the end of the light step $\left(t_{\text {end }}\right)$ (cyan line in Figure 3.10):

$$
\text { steady state }=\frac{\sum_{\text {thalf }}^{\text {tend }} r(t)}{N}
$$

where $t_{\text {half }}=t_{\text {end }}-\left(\left(t_{\text {end }}-t_{\text {peak }}\right) / 2\right)$ and $\mathrm{N}=$ number of time points between $t_{\text {end }}$ and $t_{\text {half. }}$ 
From here the sustained-transient index(STi) was computed as follows:

$$
S T i=\frac{\text { steady state }}{\text { peak }}
$$

The index takes values close to 1 for sustained cells and values close to zero for transient cells. For two cells (one example is shown in Fig.3.10D), the index took negative values, because the steady state is below zero. As one can see in Figure 3.10D, the cell is clearly transient; therefore, we put negative values as zero.

A

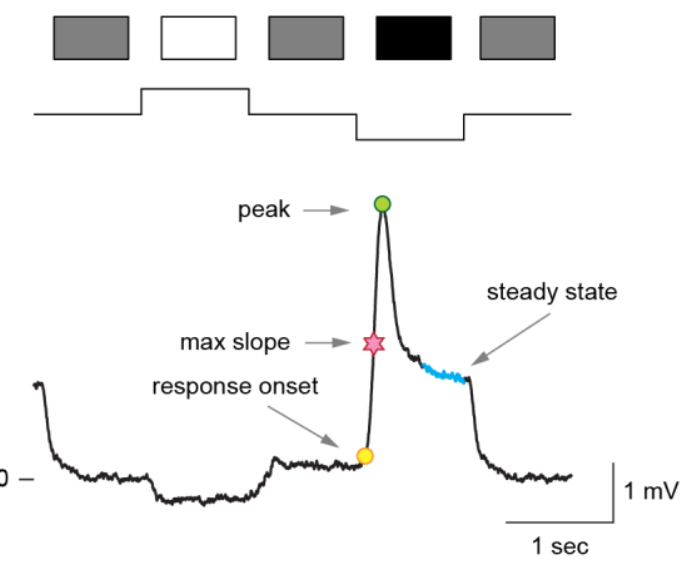

C

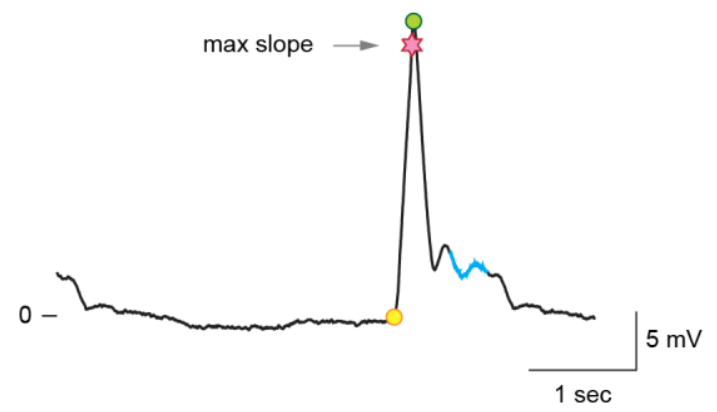

B
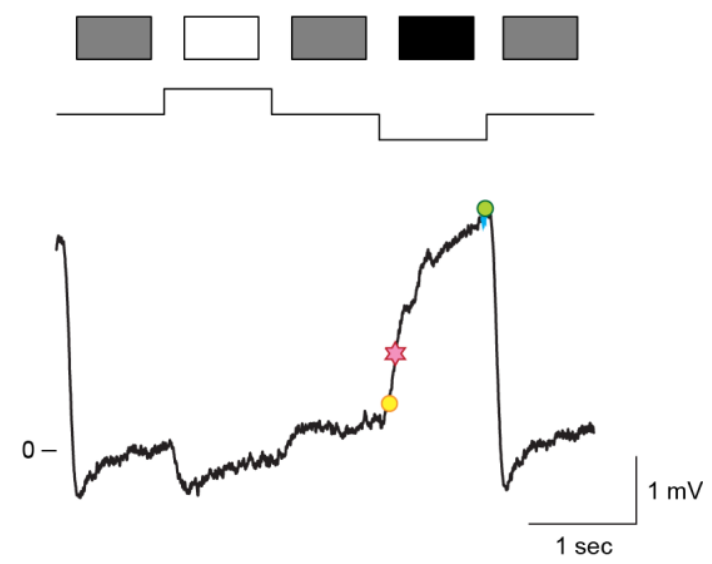

D

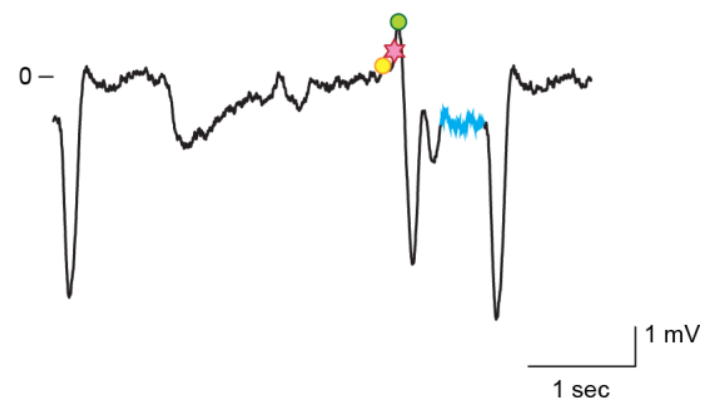

Figure 3.10. Temporal properties estimated with full-field light steps. A-D. Example response traces of bipolar cells to the full-field light step with gray frames. Yellow dot shows the onset of the response, the pink star the maximum slope of the response, the green dot is the peak of the response trace and the blue line the steady state level.

Oscillations. To quantify the oscillations observed in some responses (see Fig.3.11A for an example of an oscillatory response) the power spectrum was computed with a fast Fourier transform (FFT, using MATLAB fft function). To get the power spectrum, the square of the absolute value of the output of the FFT was taken. The frequency with the highest power was defined as the frequency at which the response is oscillating (see Fig.3.11B, the cell is responding 
with a $\sim 4 \mathrm{~Hz}$ oscillation). Note, three points had to be taken care of before the fast Fourier transformation was computed:

1. The response was cut from the peak to $50 \mathrm{~ms}$ after the end of the light step to capture the last cycle (see dashed rectangle in Fig.3.11A). The cut is crucial, if we for example take the period from the starting of the light step to the end of the light step, the maximum frequency is the $1 \mathrm{~Hz}$ response to the light step.

2. The frequency resolution depends on the amount of data we use for the fast Fourier transform (FFT). If we use $1000 \mathrm{~ms}$ of data (with a sampling rate of $1000 \mathrm{~Hz}$ ), the frequency resolution is $1 \mathrm{~Hz}$ (the frequency is determined by the Nyquist-Shannon sampling theorem $\frac{f_{S}}{N}$, where $f_{s}$ is the sampling rate and $\mathrm{N}$ the amount of datapoints). If we only use $750 \mathrm{~ms}$ of data (sampling rate stays $1000 \mathrm{~Hz}$ ), the frequency resolution is $1.333 \mathrm{~Hz}$. For cells where the peak comes very late, we were left with a small amount of data and therefore low frequency resolution. We therefore made an additional condition that we wanted to have at least a frequency resolution of $1.3 \mathrm{~Hz}$, which means at least $750 \mathrm{~ms}$ data after the peak.

3. From the sequence of interest, the overall mean was computed and subtracted. If one does not correct for the mean when computing the Fast Fourier Transform (FFT), the maximal peak is at $0 \mathrm{~Hz}$. The power at $0 \mathrm{~Hz}$ represents the mean over the sequence. If the mean is subtracted, the peak at $0 \mathrm{~Hz}$ disappears, while the other frequencies are unaffected.

A

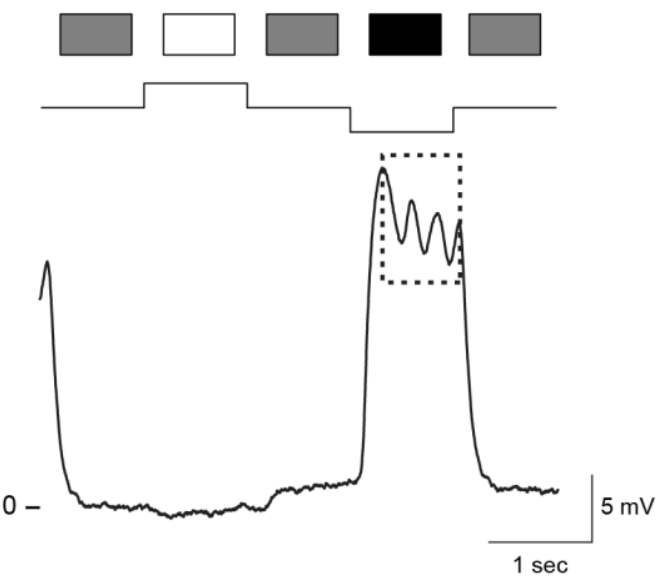

B

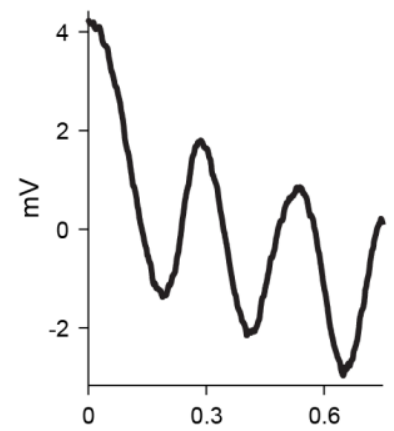

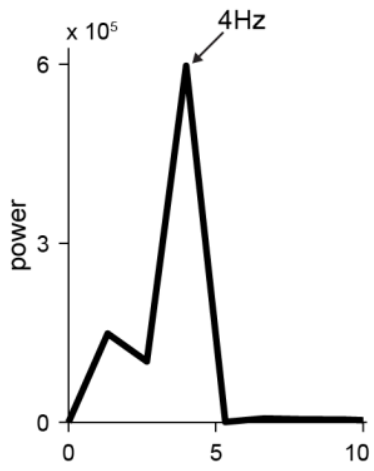

Figure 3.11. Oscillations estimated with full-field light steps. A. Example response traces of an OFF bipolar cell with oscillations. The dashed rectangle mark the start and end of the oscillations. B. Zoom into the oscillations of the dashed rectangle and corresponding power spectrum with a peak at $4 \mathrm{~Hz}$.

Spots

For the spot stimuli, the response onset, sustained-transient index and the oscillation frequency were calculated in the same way. The only difference was that the spot was shown for 
$0.5 \mathrm{sec}$ compared to the $1 \mathrm{sec}$ for the full-field flashes. Thus, for the oscillation index, $500 \mathrm{~ms}$ instead of $750 \mathrm{~ms}$ were chosen. For all analysis where we compare the indices of the spot with the full-field light steps, only the first 500ms of the full-field light step were taken into consideration.

\section{White noise}

The white noise stimulation provides another way to assess the temporal dynamics of a cell. The temporal filter of the binary white noise stimulus is computed over the singular value decomposition of the spatiotemporal receptive field (see section 3.5.2). The temporal filter of the full-field white noise stimulus is directly assessed over the computation of the voltage-triggeredaverage. The voltage-triggered-average is computed in the same way as previously described for the binary white noise stimulus (section 3.5.2), except that here, the stimulus signal has no spatial dimension.

The filters are represented with time-axis going backwards, such that time point zero indicates the response of the cell and negative values represent times farther in the past. The latency of the cell is computed by determining the time a cell needs to reach the peak of the filter (yellow dots in Fig.3.12). The time-to-peak is calculated by first fitting a parabola to three points around the maximum and then determining the time in $\mathrm{ms}$ to the peak value of the fit. Further, we also computed the time-to-rise point, which is defined as the time a cell needs to reach half of the amplitude of the peak response (red rectangle in Fig.3.12). Another common description relates to the biphasic shape of the temporal filter (Zaghloul et al., 2007). Here, we computed the absolute ratio between the amplitude of the second (green dot) and the first (yellow dot) peak. The value is close to zero, if the cell does not have a second peak (termed monophasic filter) and larger than zero, if the cell exhibit a second peak (termed biphasic filter) (compare the two cells in Fig.3.12). Usually, the biphasicness is seen as a measure for how transient the cell is. 
biphasic filter

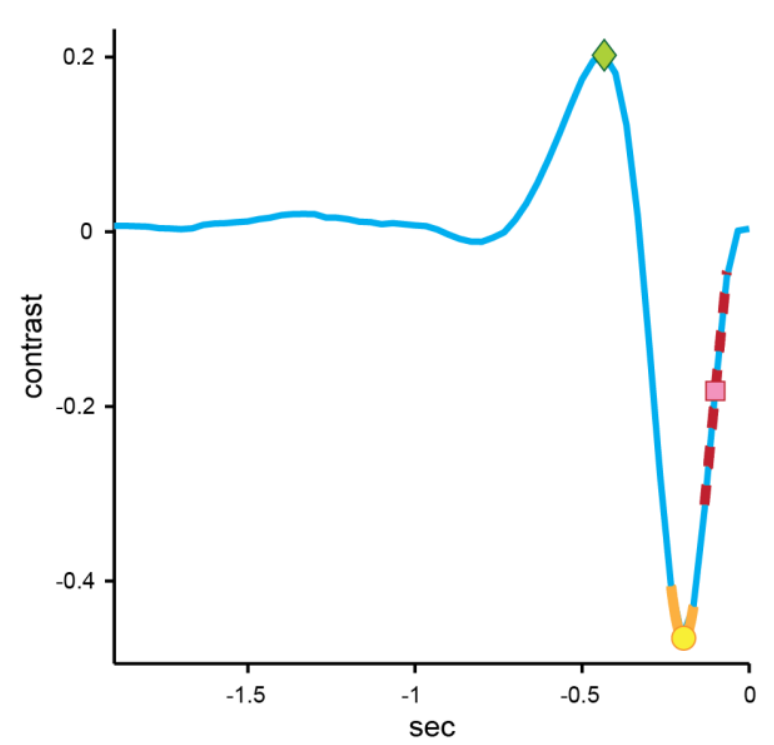

monophasic filter

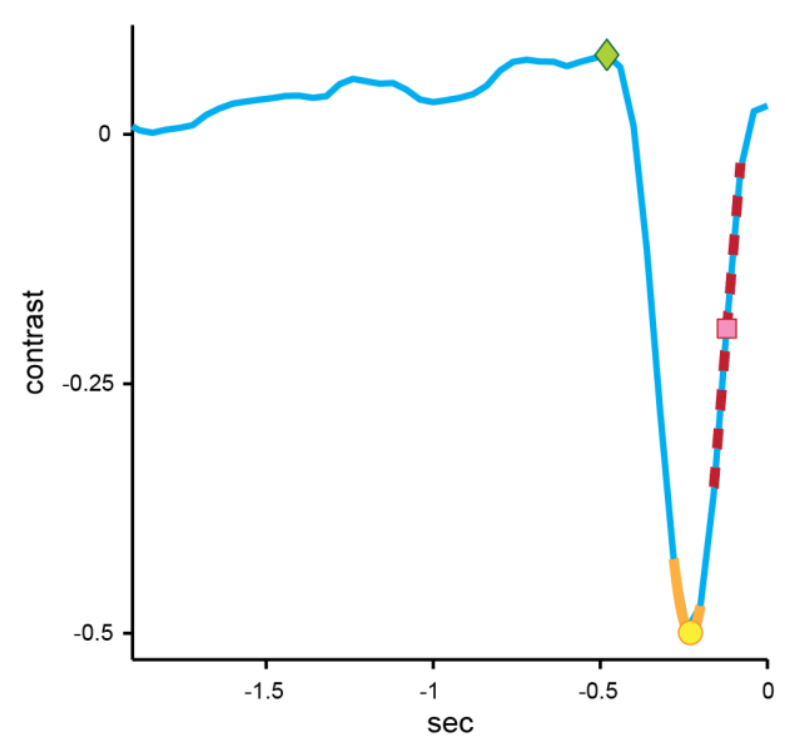

Figure 3.12. Estimating temporal properties with white noise analysis. Temporal filters of two bipolar cells. The yellow dot marks the time-to-peak, the red dot the time-to-rise and the green dot the second peak that was used to compute the biphasicness index.

\subsection{Characterization of nonlinearities}

Nonlinearities seem to play a crucial role for the response diversity observed in ganglion cells. Yet, how these nonlinearities manifest themselves in bipolar cells is unclear. In the retina, two main stages are distinguished where nonlinear signal transformation can take place: at the output and at the inputs of neurons. In our goal 2, we studied these two types of nonlinear transformations in bipolar cells by assessing the stimulus-response transformation (output nonlinearity) and the spatial integration (input nonlinearity). We describe the analysis performed for each type separately.

\subsubsection{Stimulus-response transformation}

Spots

The spots used to estimate the receptive field center-surround structure were shown with black and white contrast. For the previous analysis with spots, e.g. center-surround estimation and temporal properties, we only studied the responses to the preferred contrast (e.g. black). To study the stimulus-response transformation we investigated the response to both the preferred 
(e.g. black, $-100 \%$ ) and non-preferred (e.g. white, $+100 \%$ ) contrast. We compared the amount of depolarization to the preferred spot and the amount of hyperpolarization to the non-preferred spot (see Fig.3.13). We built a hyper-depolarization index (HDi):

$$
H D i=a b s\left(\frac{a b s\left(\max \left(r_{\text {preferred }}\right)\right)-a b s\left(\min \left(r_{\text {non-preferred }}\right)\right)}{a b s\left(\max \left(r_{\text {preferred }}\right)\right)+a b s\left(\min \left(r_{\text {non-preferred }}\right)\right)}\right)
$$

From a completely linear bipolar cell, one would expect a similar amount of depolarization and hyperpolarization to the preferred and non-preferred spot. In this case, the index takes values close to 0 . A nonlinear cell is expected to show different amounts of depolarization and hyperpolarization and here, the index takes values larger than zero.
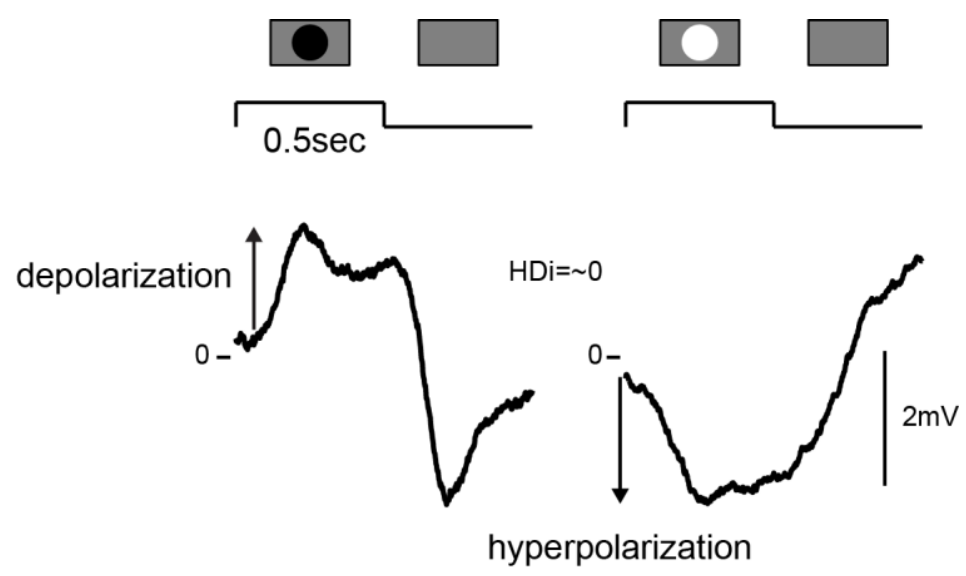

Figure 3.13. Estimation of the output nonlinearity with spots. The example bipolar cell shows a similar amount of depolarization to the preferred spot (black) and hyperpolarization to the non-preferred spot (white). The hyper-depolarization index (HDi) is in this case close to zero.

\section{White noise}

With the spots, we only studied the stimulus-response transformation for the highest contrast changes $( \pm 100 \%)$ but not for intermediate contrast values. The white noise analysis provides an approach to study those intermediate contrast values too. To do so, we assessed the stimulus-response transformation by convolving the filter (voltage-triggered average, see subchapter 3.5.2) with the original stimulus signal (see Fig.3.14). In the convolution, the filter is multiplied by a stimulus sequence of the same length as the filter and the output is summed, then the filter is shifted by $1 \mathrm{~ms}$ to the next stimulus sequence (filter2 command in MATLAB, no zero padding at edges). The convolution is a linear operation; thus, the output of the convolution is linearly proportional to the filter. For the full-field Gaussian white noise stimulus, the temporal filter was directly convolved with the stimulus signal. For the binary white noise stimulus, the 
stimulus signal was first convolved by the spatial filter and the resulting sequence was then convolved with the temporal filter. To normalize the output of the convolution, each filter was normalized to unit Euclidean norm, so that the sum of squares of the filter elements is equal to one. The filtered output was then related to the response of the cell by plotting for each convolution output ( $\mathrm{x}$-axis) the corresponding membrane potential change (y-axis) for that sequence (Fig.3.14). In the stimulus-response-transformation pictured in Figure 3.14, the cell increases the membrane potential by $10 \mathrm{mV}$ (depolarization, compared to baseline level) when the incoming stimulus sequence matches the cell's filter (green shaded region and dot). Further, the cell is silent ( $0 \mathrm{mV}$ change from the baseline) when the stimulus sequence is opposite to the filter (orange shaded region and dot).

To quantify the degree of nonlinear transformation, the convolution output was binned into 40 bins and each bin contained the same number of data points. Then, the average convolution output was plotted against the average membrane potential change for each bin (blue dots in Fig.3.14). From here, a nonlinearity index was built by fitting a straight line to the negative (blue line) and positive (red line) parts of the output function (Fig.3.14). The slopes of the negative and positive values were then compared and an output nonlinearity index (ONi) was computed:

$$
O N i=a b s\left(\frac{\text { slope }_{\text {positive }}-\text { slope }_{\text {negative }}}{\text { slope }_{\text {positive }}+\text { slope }_{\text {negative }}}\right)
$$

An index close to 0 means that the negative and positive slopes are almost identical and therefore the cell shows overall a very linear transformation to the input light stimulus. A value larger than 0 means that the cell shows a different transformation for positive compared to negative filtered stimulus values (Fig.3.14). Note, for some analysis (Fig.5.4F), we used the non-absolute value of the $\mathrm{ONi}$, because we were interested in the direction of the nonlinearity (directional $\mathrm{ONi}$ ). In this case, a positive index means that the cell responds more strongly to the positive filtered signals compared to negative filtered signals; a negative index means that the cell shows a saturation for positive filtered signals. 


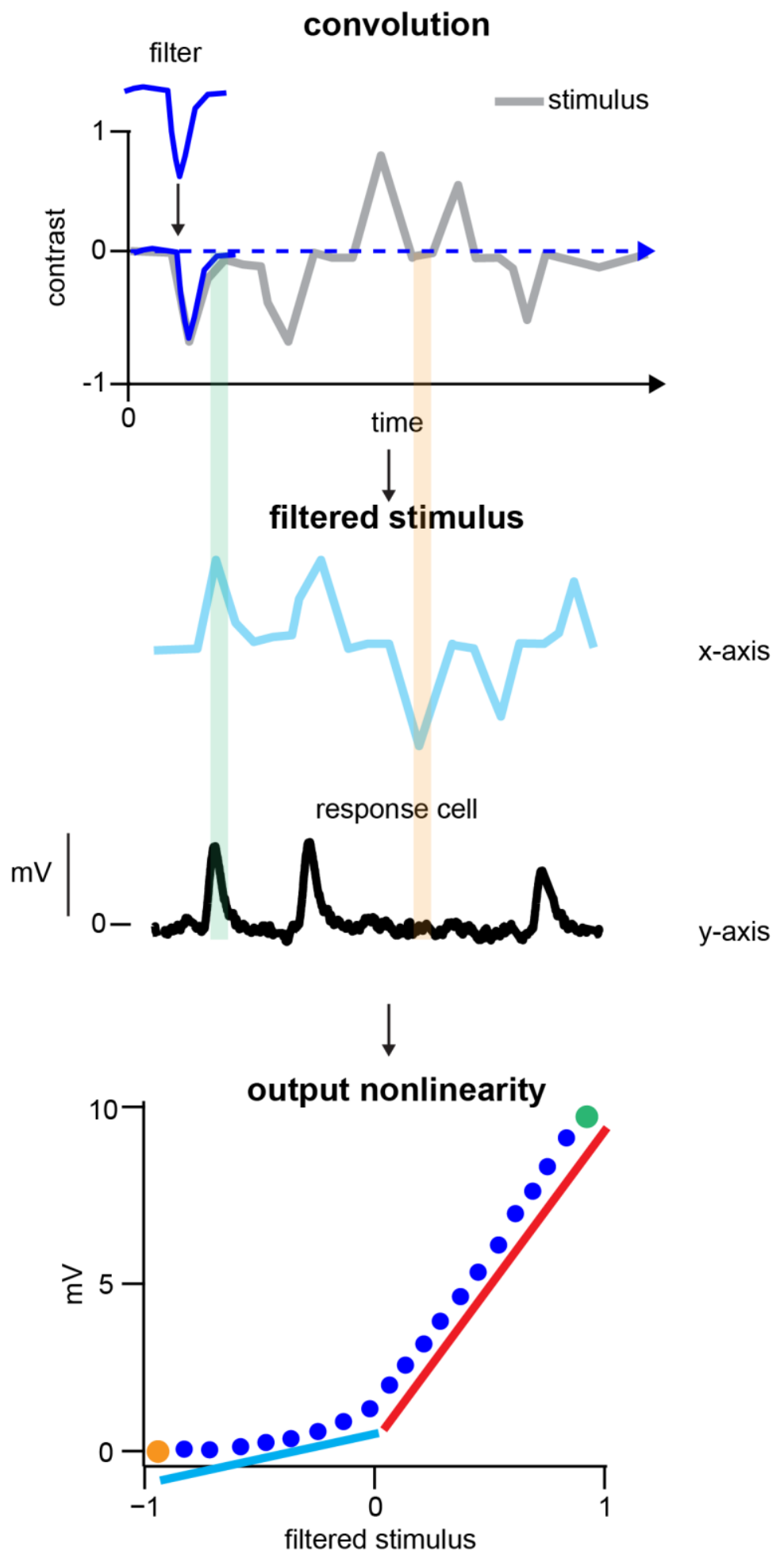

Figure 3.14. Estimation of the output nonlinearity with white noise. The input stimulus signal (contrast) is first filtered by the cell's internal filter (convolution). Then, the filtered stimulus is compared to the response of the cell to build the output nonlinearity (see green and orange colors). For example, the stimulus signal that is similar to the filter leads to a high convolution output and is mapped to the corresponding membrane potential change of $10 \mathrm{mV}$ (green shaded region and dot). The output nonlinearity is quantified by fitting a straight line to the positive and negative filtered stimulus signal. 


\subsubsection{Spatial integration}

The second type of nonlinearity we studied in goal 2 was the spatial integration. Here, we investigated whether bipolar cells integrate input signals in a linear or nonlinear way. We studied the integration in the receptive field center (spot grating) as well as under surround stimulation (full-field grating). The two stimulus designs are shown in Figure 3.15.

\section{grating spot}
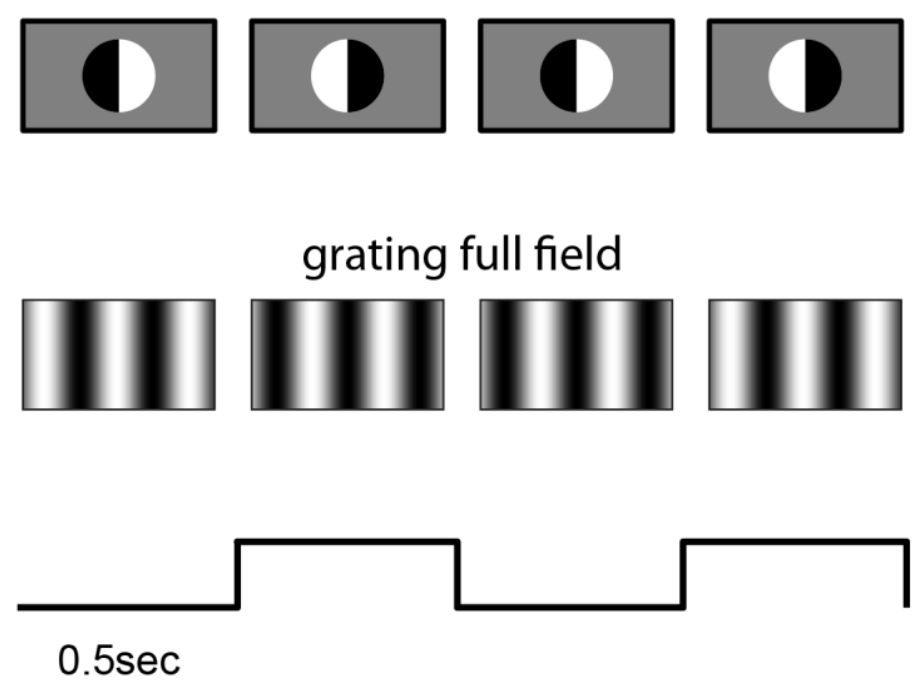

Figure 3.15. Stimulus designs to study spatial integration.

\section{Grating spot}

First, the location of the receptive field center was determined by flashing a spot over the retina (see subchapter 3.5.2 for details). At the determined location, spots with different diameters were presented and the diameter that maximally activated the cell was defined as the receptive field size. Then, various spot gratings were presented at the defined location and diameter size. 3-4 types of spatial patterns were shown to the cells. We separated the receptive field into two halves (Fig.3.15), into four quarters, into squares of $25 \mu \mathrm{m}$ and for some cells squares of $10 \mu \mathrm{m}$. The polarity of the contrast in the patterns was periodically reversed at a $1 \mathrm{~Hz}$ rhythm for 4 seconds (4 trials). The different spatial gratings were shown in sequence, starting from the largest (halves) to the smallest ( 25 or $10 \mu \mathrm{m}$ ) pattern. Between the spatial gratings, a gray period was presented for 4 seconds.

As for the spots, we computed the average baseline activity by building the mean over the membrane potential of the last $200 \mathrm{~ms}$ for each gray period. The response traces for each grating type was baselined by subtracting the corresponding baseline level. We then built per grating type an average trace over trials. 
Hochstein and Shaple (1976b) were among the first who objectively quantified the degree of nonlinearity in the response to gratings with Fourier analysis. The idea is that if a cell nonlinearly integrates input signals, the grating and its reversal would lead to two responses, which in the power spectrum can be seen as a peak at the double of the stimulus frequency. In our case the stimulus frequency is $1 \mathrm{~Hz}$, thus the double is $2 \mathrm{~Hz}$. A cell on the other hand that does not respond to the grating and its reversal would not show a peak at $2 \mathrm{~Hz}$. Similar to their work, we quantified the response to the grating by computing an index for nonlinear spatial integration. Therefore, we compared the response to the grating with a response to a black-white flashing spot (1Hz frequency, Fig.3.16A\&B). The black-white flashing spot provides a control condition to assess the maximal possible response of the cell and the corresponding maximal expected power at the stimulus frequency $(1 \mathrm{~Hz})$. We computed a fast Fourier transform (FFT, using Matlab's fft function) on the average response traces of the grating and the black-white flashing spot (Fig.3.16). For the flashing spot, the spectrum has a peak at $1 \mathrm{~Hz}$, which is the modulation frequency of the spot. For the grating, the power spectrum exhibited a peak at $2 \mathrm{~Hz}$, thus, double the stimulus frequency (see gray dots in Fig.3.16). Note, for 2 out of 23 cells, we observed a peak in the power spectrum at $4 \mathrm{~Hz}$ for the reversing grating (see Fig.3.16B). The $4 \mathrm{~Hz}$ response becomes understandable when looking at the response to the flashing spot. Here, the cell showed an oscillatory response of two peaks. When presenting the reversing grating, the cell continued responding to each reversals of the grating with two peaks, which leads to the $4 \mathrm{~Hz}$ response.

We computed the spatial nonlinearity index as a ratio between the sum of the power at 2 and $4 \mathrm{~Hz}$ from the reverse grating and the power at $1 \mathrm{~Hz}$ from the flashing spot:

$$
\text { spatial nonlinearity index }=\frac{\left(\text { power }_{2 \mathrm{~Hz} \text { grating }}+\text { power }_{4 \mathrm{~Hz} \text { grating }}\right)}{\text { power }_{1 \mathrm{~Hz} \mathrm{Spot}}}
$$

Cells with a spatial nonlinearity index (SNi) close to 0 do not respond to the reversing grating, while cells with indices above 0 respond to the reversing grating. 
A Cell 1
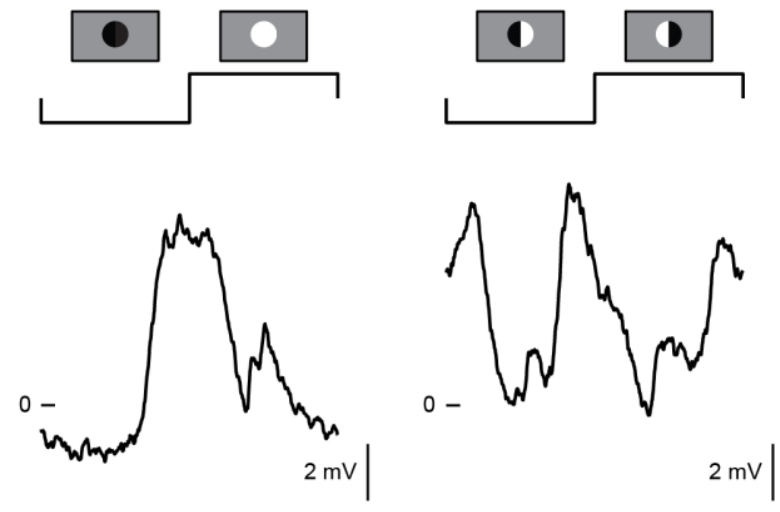

power spectrum
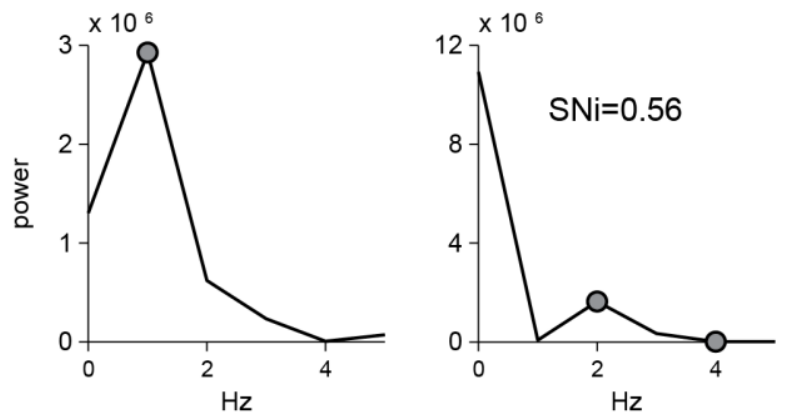

B

\section{Cell 2}
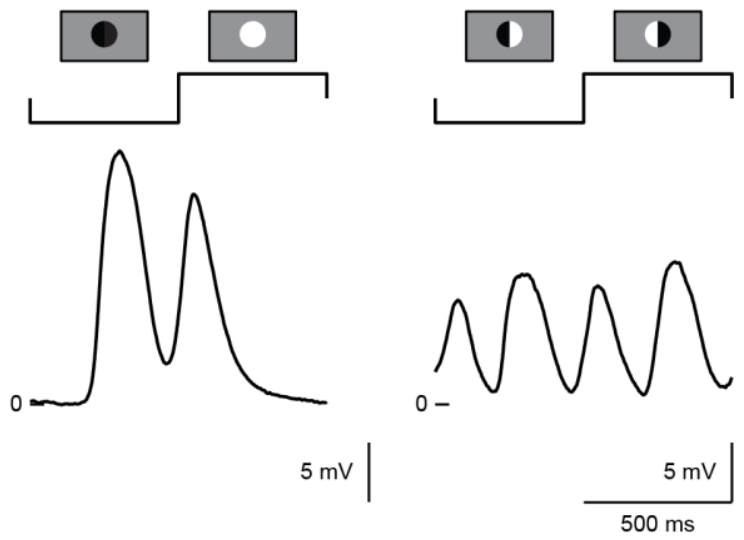

power spectrum
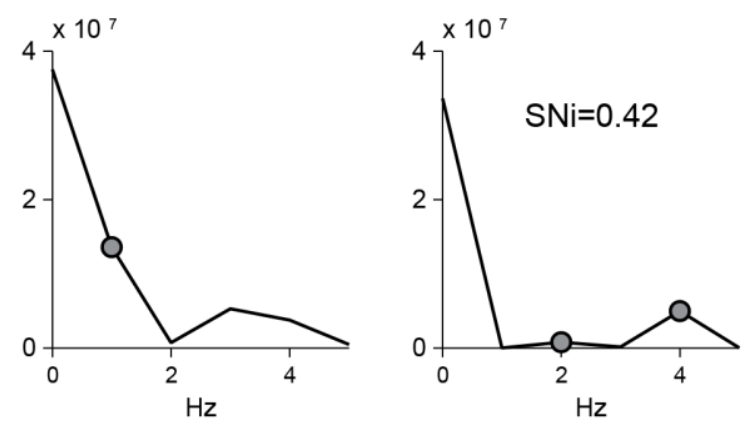

Figure 3.16. Estimation of nonlinear spatial integration. A. Example cell with the response traces to the blackwhite spot and the reversing spot grating. Below is the corresponding power spectrum. The black-white spot leads to a peak at $1 \mathrm{~Hz}$, the reversing grating to a peak at $2 \mathrm{~Hz}$. The spatial nonlinearity index $(\mathrm{SNi})$ is $\sim 0.56$. B. An example cell that showed a peak at $4 \mathrm{~Hz}$ to the reversing grating.

\section{Full-field grating}

To investigate, whether the spatial integration is changing under surround stimulation, we presented to some recorded bipolar cells a reversing full-field grating. We used bar width of 40, 80 and $150 \mu \mathrm{m}$. The grating was reversed every $500 \mathrm{~ms}$ and after 8 trials, the spatial phase was shifted by $45^{\circ}$. We baseline-corrected the recorded traces by the mean of the last $200 \mathrm{~ms}$ of the first gray period shown at the beginning of the light stimulation and built the average over the trials for each spatial phase. We again computed the fast Fourier transformation of the average response trace per spatial phase (Hochstein and Shapley, 1976b). Different from the grating spot, we compared the maximum frequency at $2 \mathrm{~Hz}$ to the maximum frequency at $1 \mathrm{~Hz}$ over all the spatial phases:

$$
\text { spatial nonlinearity index }=\frac{\max \left(\text { power }_{2 \mathrm{~Hz}}\right)}{\max \left(\text { power }_{1 \mathrm{~Hz}}\right)}
$$




\subsection{Linear-nonlinear model}

For the third goal, we studied the responses of bipolar cells with the linear-nonlinear model. The model assumes a linear signal transmission from photoreceptors to bipolar cells and if the assumption holds, an accurate prediction of bipolar cells' responses can be expected. The linear-nonlinear model is a cascade model that comprises two stages: a filter and a nonlinear transformation. The filter is the voltage-triggered-average that we described in subchapter 3.5.2. The nonlinear transformation is the nonlinear output function, with the convolution output at the $\mathrm{x}$-axis and the membrane potential at the y-axis, as described in subchapter 3.6.1. In the model, neurons linearly filter the input signals and transform the output through a nonlinear function to a response. To study how accurate the model describes the real response of a neuron, the model is built with a training set (e.g. randomized contrast sequence) and the response to a test set (e.g. fixed contrast sequence) is predicted. The prediction is done by convolving the filter with the test set and reading the predicted responses from the nonlinearity of the model. Finally, the performance of the model is assessed by comparing the real response of the cell with the predicted response. We investigated the performance of the model with three different stimulus sets: full-field white noise, binary white noise and natural movies. In this chapter, we look at the mathematical implementation of the model for the different light stimuli.

\subsubsection{Full-field white noise}

We first studied the performance of the model with full-field white noise (Fig.3.17A). The contrast sequence was composed of one non-repeated sequence (gray trace, termed running noise, Fig.3.17A), the training set, that was independently drawn from a Gaussian distribution. The sequence was regularly interrupted (every 30 seconds) by the same contrast segment of 10 seconds (Fig.3.17A, red traces, termed frozen noise). The repeated contrast segment built the test set that was at the beginning also randomly drawn from a Gaussian distribution. The full-field white noise stimulus was shown for $\sim 10$ minutes and as previously described; slow fluctuations in the membrane potential were removed by de-trending the data. The test set was repeated $\sim 10$ 15 times and the responses to the test set were averaged.

The linear-nonlinear model was built with the training set (not test set!); the filter and nonlinearity were deduced as previously described (see Fig. 3.17A). The test set was used to evaluate the performance of the model. To do so, the test set was convolved with the built filter and the output provided the new input $\mathrm{x}$-values for the nonlinearity of the model. The membrane potential change was predicted by fitting a straight line to the two closest bins, one that is larger 
and the one that is smaller from the new $\mathrm{x}$-value, and from here, the new predicted membrane potential was interpolated. For new x-values that were at the most right or left side of the output function, the membrane potential was extrapolated by fitting a straight line to the two bins closest to the $\mathrm{x}$-value. To evaluate the performance of the model the real averaged response of the test set (black trace) was compared to the predicted response (red trace) (Fig.3.17A). The model performance was quantified by computing the explained variance $\left(\mathrm{R}^{2}\right)$ defined as the square of the correlation coefficient $\mathrm{R}$ between the predicted and measured membrane potential response.

A

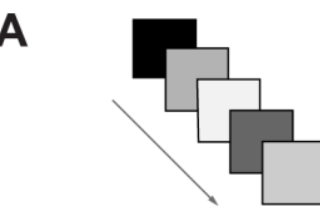

\section{full-field white noise}

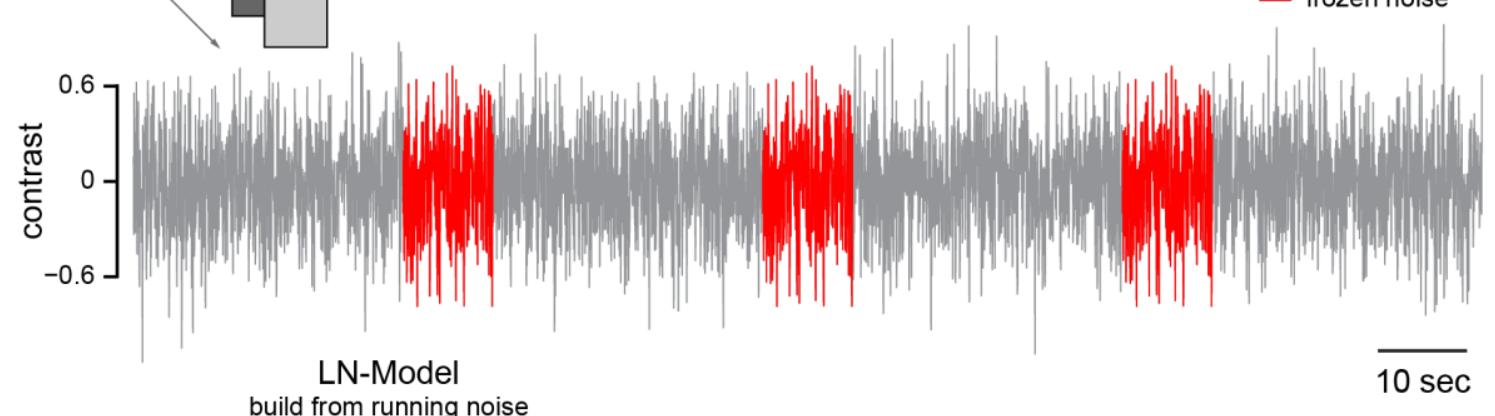

build from running noise
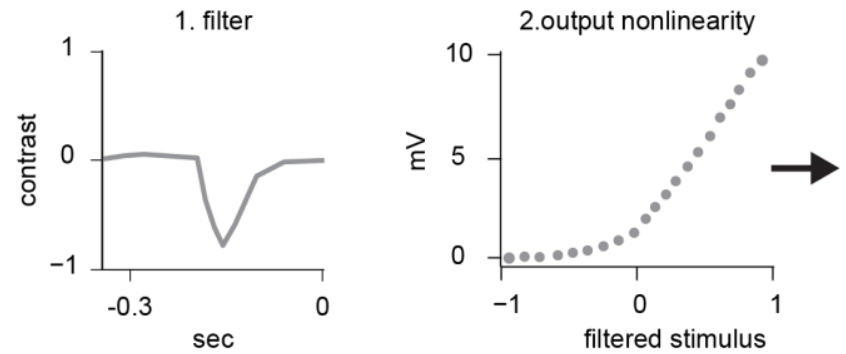

Prediction

response to frozen noise

B

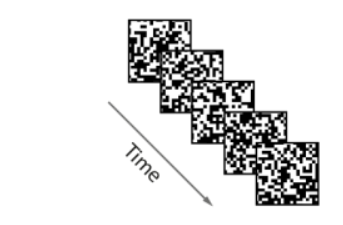

binary white noise

1. filter

LN-Model

build from training set

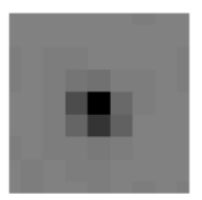

2. output nonlinearity

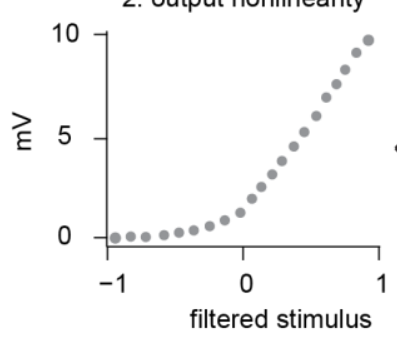

Prediction response to test set
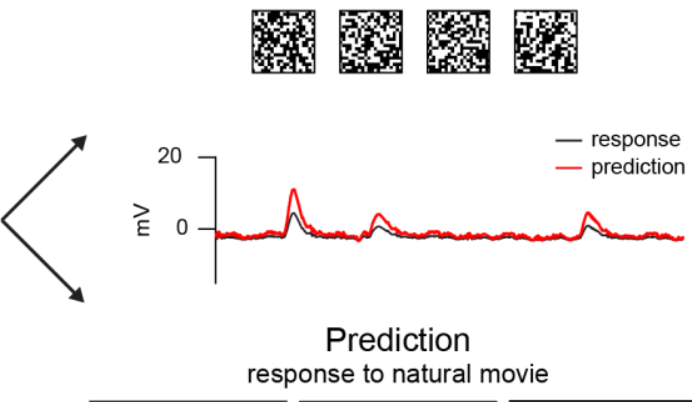

Prediction

response to natural movie
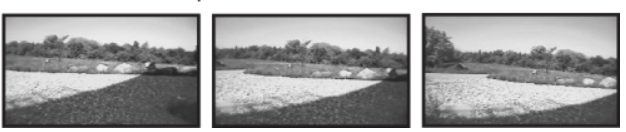
Figure 3.17. Implementation of the linear-nonlinear model for different light stimuli. A. Full-field white noise stimulus with the running (training) and frozen (test) noise sequences. The filter and nonlinearity of the linearnonlinear model are built with the training set. The membrane potential to the frozen noise sequence (test set) is then predicted and compared to the real response. B. Model built with a training set of the binary white noise stimulus. The response of a test set is then predicted and compared to the real response. The test set was either a binary white noise sequence or a natural movie. The natural movies were selected from the standardized "CatCam" database (Betsch et al., 2004).

\subsubsection{Binary white noise}

The binary white noise stimulus was one sequence of contrast values without repeatedly inserted segments (Fig.3.17B). The separation into a test and training dataset was therefore done by a leave-one-out technique. Randomly, 200 segments of 10 seconds length were chosen from the white noise contrast sequence (test set). Then, the linear-nonlinear model was built 200 times by always leaving out the sequence that is being predicted. For each predicted sequence, the real response of the cell was compared to the predicted response by computing the explained variance $\left(R^{2}\right)$. The final performance of the model was calculated as the average over all the computed values of the explained variance.

To predict responses to the binary white noise stimulus, we only analyzed the bipolar cells with a good receptive field (maximum pixel at least two standard deviation above the noise). Further, we only considered bipolar cells with a membrane potential signal of at least $0.5 \mathrm{mV}$ for one standard deviation.

\section{Overfitting in the linear-nonlinear model}

For the binary white noise analysis, we ran into an unexpected problem of overfitting. The problem is illustrated in Figure 3.18A. When using all the pixels of the screen, the prediction to the test dataset, the dataset that was not used to build the model, was very low (14\%). Yet, for the prediction to the training dataset, the dataset that was used to build the model, the performance was very high $(\sim 87 \%)$. Overfitting describes the phenomena of a model that almost perfectly describes the data it was built on, but fails to generalize to a new dataset. The idea is that the model has no predictive power and does not describe the underlying structure of the data. To understand overfitting in an intuitive way, let us imagine we learn by heart chemical formulas without understanding the rules behind them. In such a case, we would have a very high grade if in a school test the exact same formulas were requested. Yet, if we have to transfer the knowledge and build new formulas, we would fail. 
To avoid overfitting, we reduced the amount of variables in our spatiotemporal filter and cut a region around the pixel with the maximum activity (Fig.3.18B, 3 sigma cut). By reducing the variables, the prediction to the test dataset became similar to the prediction of the training dataset (Fig.3.18B). Further, the singular value decomposition additionally reduced the noise of the surrounding pixels and helped to avoid overfitting.

We also tested whether fitting a function to the original data points of the output function (before binning) or the bins themselves increased the model performance and reduced overfitting. Here, we found that the described linear interpolation between the bins led to the highest prediction performance and lowest overfitting. 


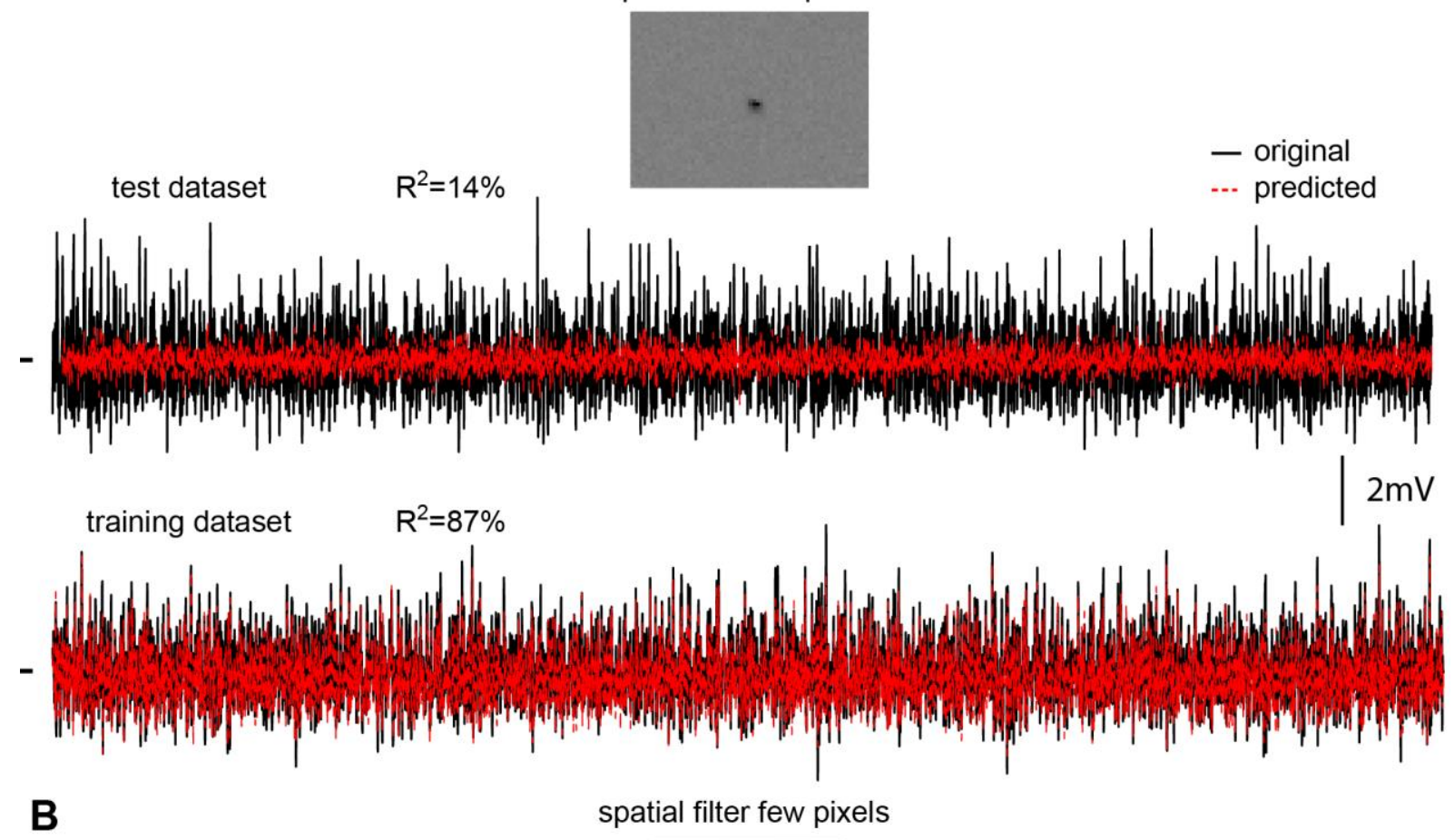

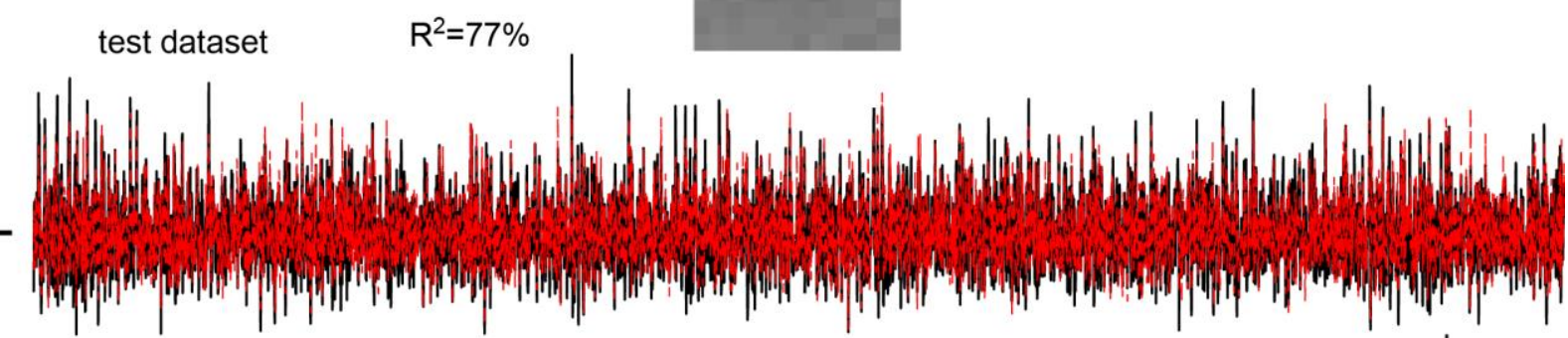

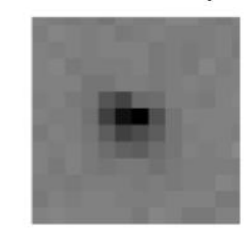

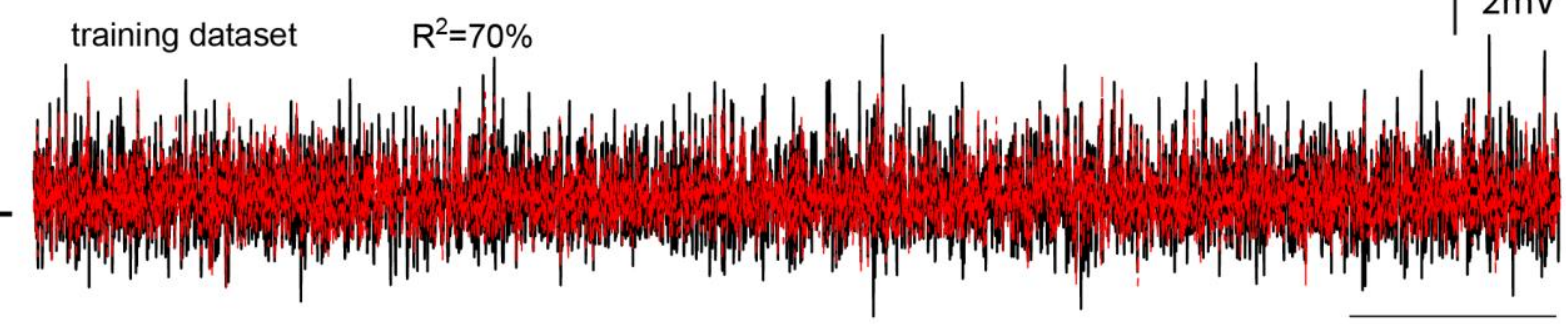

$1 \mathrm{~min}$

Figure 3.18. Overfitting in the linear-nonlinear model. A. All the pixels of the spatiotemporal filter were used to predict the response of the bipolar cell. For the test dataset, the prediction failed (14\%), yet for the training dataset the prediction was high $(87 \%)$. B. When reducing the amount of pixels in the spatiotemporal filter, the prediction performance was good for both test and training dataset ( $77 \% \mathrm{vs}$. $70 \%)$. 


\subsubsection{Natural movies}

We showed to the bipolar cells natural movies and recorded their responses (see example frames of one movie in Figure 3.17B). The natural movies were selected from the standardized "CatCam" database (Betsch et al., 2004) that has been used for ganglion cells (Katz et al., 2016), visual cortex (Kayser et al., 2003) or lateral geniculate nucleus (Mante et al., 2008). In the "CatCam" database, natural scenes were recorded from a cat's perspective. Equipped with a camera on their head, cats were exploring diverse outdoor environments (Betsch et al., 2004). In the movie in Figure 3.17B for example, cats were exploring an environment with stones and grass. Five different natural movies were used, three of them with a length of $\sim 40$ seconds $(25 \mathrm{~Hz})$, two of them with a length of $\sim 20$ seconds $(25 \mathrm{~Hz})$. Each movie was repeated $\sim 10$ times.

The two stages of the linear-nonlinear model (i.e. filter and stimulus-response function) are built from the responses to the binary white noise stimulus (see Fig.3.17B). Then, we convolved the filter to the natural movie sequence and put the convolution output into the stimulus-response function. From here, we predicted the responses to the natural movies (red dashed trace in Fig.3.17B). Again, we evaluated the performance of the model by computing the explained variance.

To control for adaptation during the exposure of the movie, we also computed the linearnonlinear model with the stimulus-response function of the movie. To do so, we plotted the convolution output from the white noise filter against the response of the movie for the corresponding sequence. From here, the prediction and performance were computed as described before. 


\section{General characterization of}

\section{bipolar cells}

In our goal 1, we aimed to describe bipolar cells based on classical response properties. The characterization included the response polarity (subchapter 4.1), the receptive field center (subchapter 4.2) and surround structure (subchapter 4.3) and the temporal dynamics of the response (subchapter 4.4). Characterizing the bipolar cells' responses is an important first step before studying their nonlinearities and model their responses.

\subsection{Response polarity}

We started with the most fundamental and classical characterization of bipolar cells: their response polarity. To do so, we used classical full-field light steps and characterized whether bipolar cells responded to light increments (ON cells) or light decrements (OFF cells). The fullfield light stimulus is illustrated in Figure 4.1. For each trial, the screen changed from a uniform gray to either white $(100 \%$ contrast increase) or black (100\% contrast decrease). Figure 4.1A shows the response of an OFF bipolar cell, both the original single trial traces and the average relative membrane potential change. The OFF bipolar cell responded with an increase in the membrane potential (depolarization, relative to the gray light step) to the light decrements (black screen) and with a decrease in the membrane potential (hyperpolarization, relative to the gray light step) to the light increments (white screen). The ON bipolar cells responded in the opposite direction, for example the cell in Figure 4.1B depolarized to the white screen and hyperpolarized 
to the black screen. In total, around $92 \%$ of the recorded bipolar cells were OFF cells, while only $8 \%$ were ON cells (Fig.4.1C, $\mathrm{n}=51$ ). No clear ON-OFF cells were observed. The strong bias towards OFF cells is commonly observed in our lab group for the axolotl retina and has been reported in the tiger salamander both for bipolar cells (Hare et al., 1986; Hare and Owen, 1990) and for ganglion cells (Burkhardt et al., 1998; Segev et al., 2006).

A

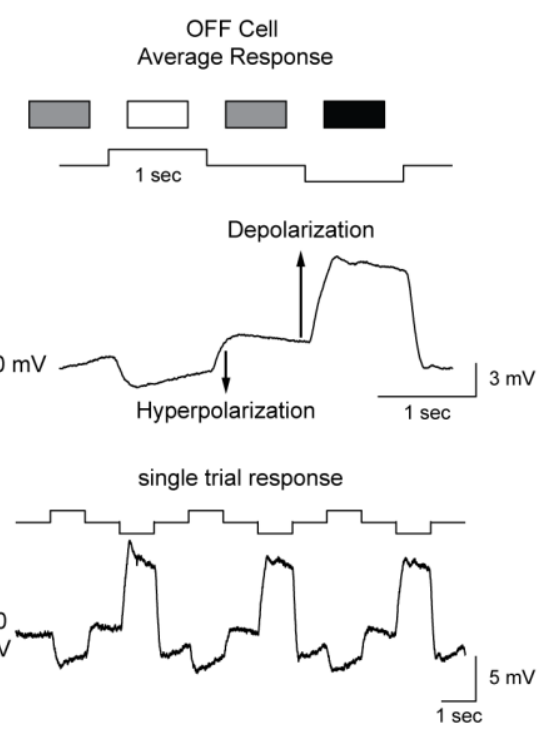

B
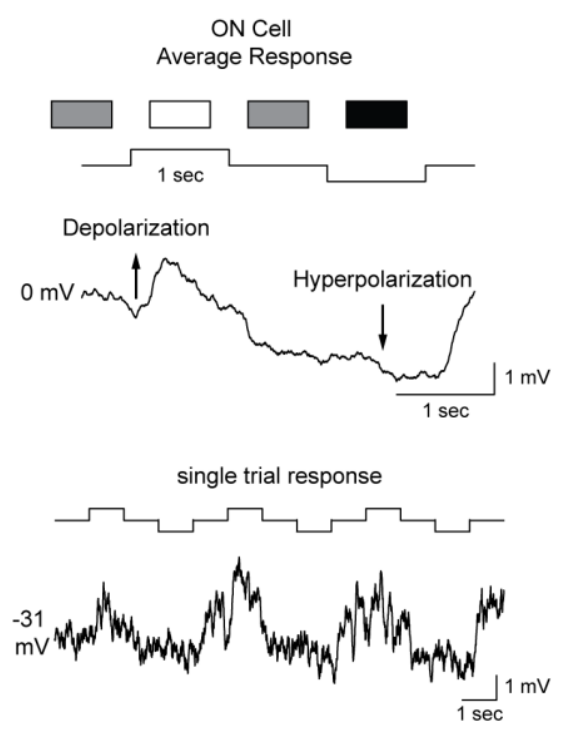

C

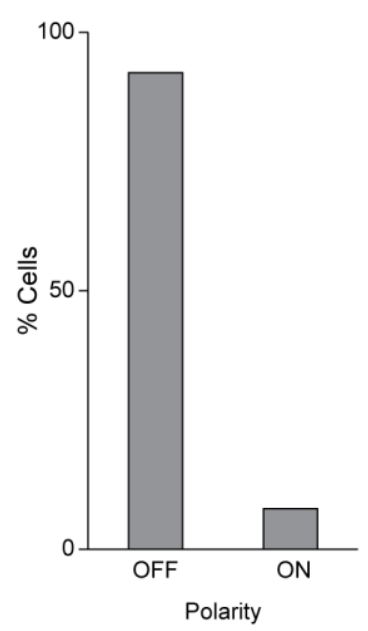

Figure 4.1. Response polarity. Example traces to the full-field light steps and population distribution for the response polarity. A. Shows the average and single trial responses of an OFF cell. B. Shows the average and single trial responses of an ON cell. C. Shows the $\%$ of ON and OFF cells ( $n=51)$.

\section{Excurse: Gray or no gray?}

From the 51 recorded cells in Figure 4.1C, 11 were recorded with a light step that switched between a black and a white screen, thus without an intermediate gray light step (see Figure 4.2A for the stimulus design). In our early recordings, we used the black-white switch stimulus, because it is a common stimulus to determine response polarity (see for example Baden et al. (2013a)). We realized however that for cells recorded with the black-white switch stimulus, the responses to the preferred contrast (e.g. white screen) were confounded with the responses to the non-preferred contrast (e.g. black screen). The effect is illustrated on an example cell in Figure 4.2. The response of the cell to the black-white switch in Figure 4.2A can be interpreted as if the cell responded to the white screen. However, when comparing the response to the stimulus with a gray intermediate screen, one sees that the cell did not respond to a change from gray to white, the cell only depolarized to a change from black to gray (4.2B). Therefore, the responses to the stimulus with a gray intermediate screen contain more information about the contrast preference of the cell, i.e. the cell does not respond to all kinds of increment of light; it depends 
on the starting level (black vs. gray). Similar to other studies, we therefore changed the light stimulus and used an intermediate gray screen to determine the response polarity (TikidjiHamburyan et al., 2015).

A

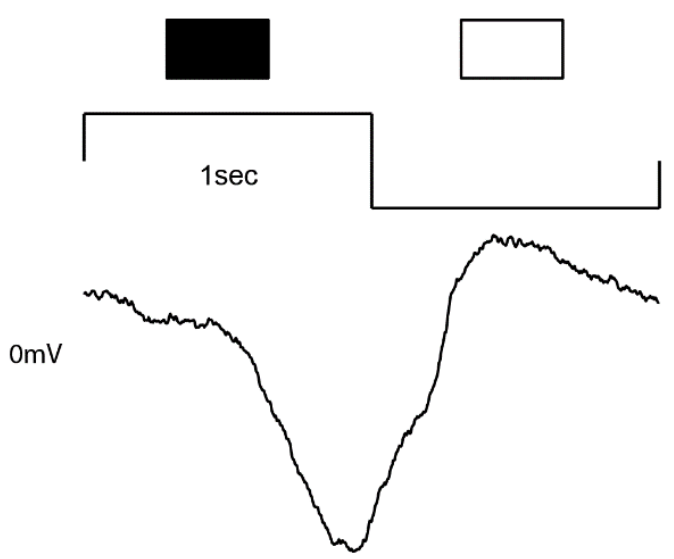

B
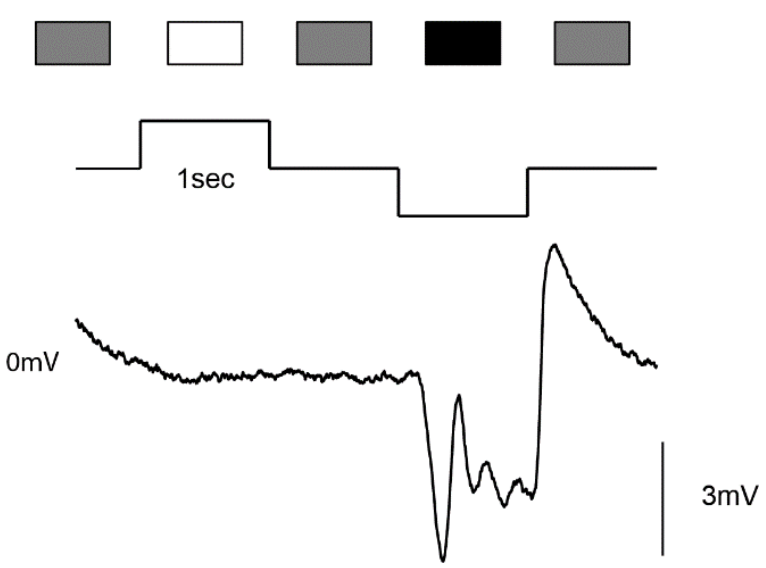

Figure 4.2. Investigating the response polarity with gray preframes. Response traces of the same ON cell to light steps with and without intermediate gray screen. A. Shows the average response trace to the black-white switch stimulus. B. Shows the average response trace of the same cell to the light step stimulus with intermediate gray steps.

\subsection{Receptive field center estimation}

Classically, the receptive field center is measured with spots that change between different diameter sizes. Another way to estimate the receptive field center is by a binary white noise stimulus. Binary white noise is a standard method to measure the receptive fields of ganglion cells. For bipolar cells the receptive fields are traditionally measured with spots, yet recently, also binary white noise has been used (Baccus et al., 2008; Franke et al., 2017). We measured the receptive field center both with classical spots (section 4.2.1) and with the binary white noise stimulus (section 4.2.2) and compared the estimated sizes (section 4.2.3).

\subsubsection{Center estimation with spots}

To estimate the center size with spots, varying spot sizes were presented over the location of the bipolar cell (see method 3.5.2). The spot changed between the preferred contrast (black or white) and gray. Figure 4.3A shows the responses of an OFF bipolar cell to different spot diameters and the corresponding tuning curve. The OFF bipolar cell showed a clear optimal 
response to a diameter of $80 \mu \mathrm{m}$ (arrow in Fig.4.3A). Around 87\% of the recorded bipolar cells showed optimal responses to diameters between 80-500 $\mu \mathrm{m}(\mathrm{n}=23)$. Surprisingly, the other 13\% of the recorded bipolar cells continuously increased their response with increasing spot sizes and reached their maximum response at the largest spot diameter of $1000 \mu \mathrm{m}$ (example in Fig.4.3B). Figure 4.3C shows the population distribution of the estimated receptive field sizes that varied between 80 and $1000 \mu \mathrm{m}$.

A
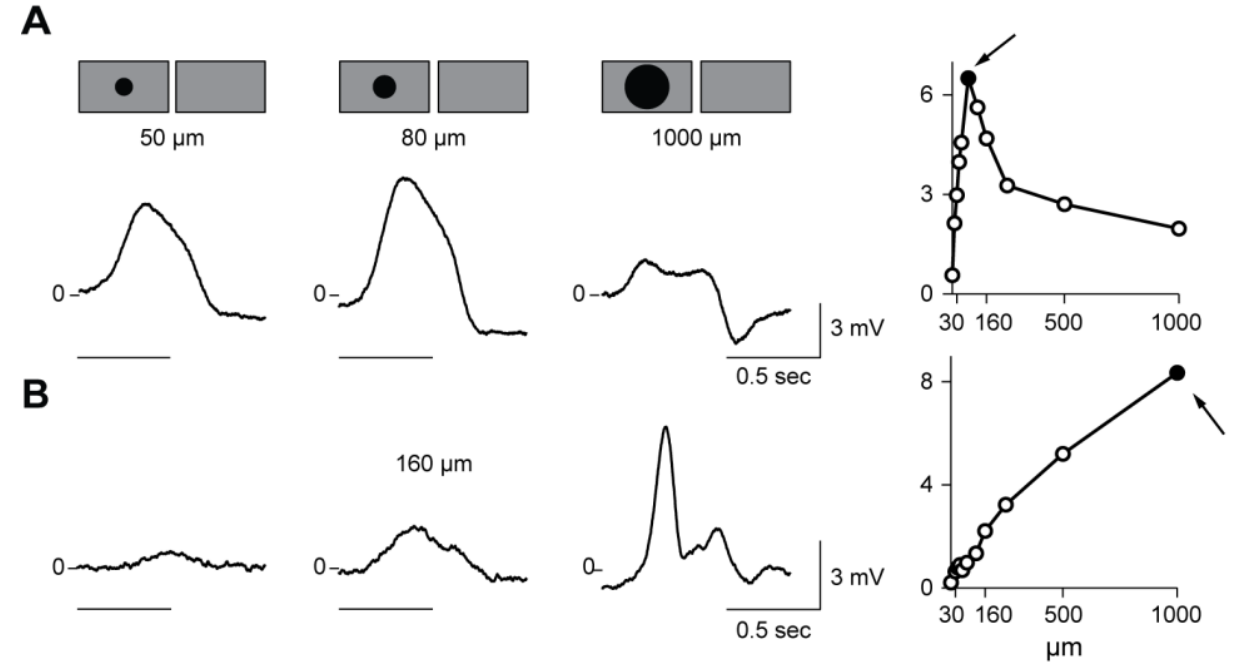

C

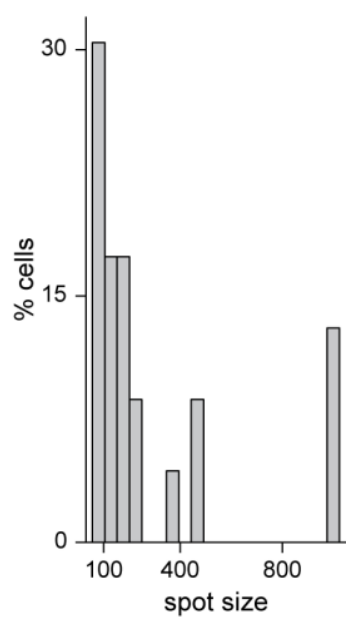

Figure 4.3. Center estimation with spots. A \& B. Response traces of OFF bipolar cells to spots with different diameters and the corresponding tuning curve. A. The bipolar cell showed a clear optimal response to a spot size of $80 \mu \mathrm{m}$ (arrow and black dot). B. Example of a bipolar cell who did not show a clear optimal response to any spot size; it reached its maximum with the largest spot diameter $(1000 \mu \mathrm{m})$. C. Population distribution of the estimated center diameter with spot stimulus $(n=23)$.

Studies in the salamander retina measuring the receptive field size with spots, reported diameters between 272-515 $\mu \mathrm{m}$ (Fahey and Burkhardt, 2003) and 100-500 $\mu \mathrm{m}$ for cone bipolar cells (Burkhardt et al., 2011)and 374-662 $\mu \mathrm{m}$ for rod bipolar cells (Borges and Wilson, 1987). Thus, our receptive field sizes between 80 and $500 \mu \mathrm{m}$ for $87 \%$ of our cells seem in the line with these studies. For the additional three cells that continuously increased their response even with spots of $1000 \mu \mathrm{m}$, the receptive fields seem too large. Such a continuous increase could be explained by a mismatch between the "real" position of the cell and the determined position (Van den Bergh et al., 2010). To test this hypothesis, we need to estimate "real" position of the receptive field center and compare it to the determined spot position. As mentioned in the introduction of this subsection, the binary white noise stimulus, introduced in the next section, is another way to measure the receptive field. 


\section{Excurse: Gray or no gray?}

From the 23 recorded cells in Figure 4.3C, 9 were recorded with spots of different sizes that switched between black and white, thus without an intermediate gray screen (see Fig.4.4 for the stimulus design). Some studies measure the receptive field size with an intermediate gray screen (Fahey and Burkhardt, 2003) others do not use a gray screen (Borghuis et al., 2013). For seven cells, we recorded both stimuli and therefore tested whether the different stimulus designs had an effect on the center size of the same cell. We did not find a significant difference between the size estimated with the spot with gray screen and the size estimated with the black-white switching spot $(p=0.24, n=7)$. Furthermore, we also found that the continuous increase in the response amplitude for larger spot sizes was still present with spots that changed between black and white (Fig.4B). We therefore excluded the possibility that the additional intermediate gray light steps can change the center diameter size.

A
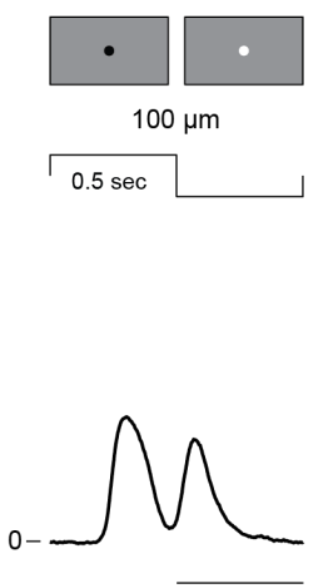

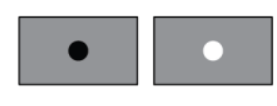

$200 \mu \mathrm{m}$

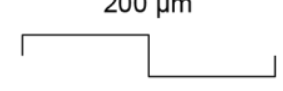

$\int_{0-\infty}$

0

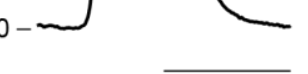

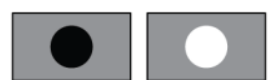
$500 \mu \mathrm{m}$

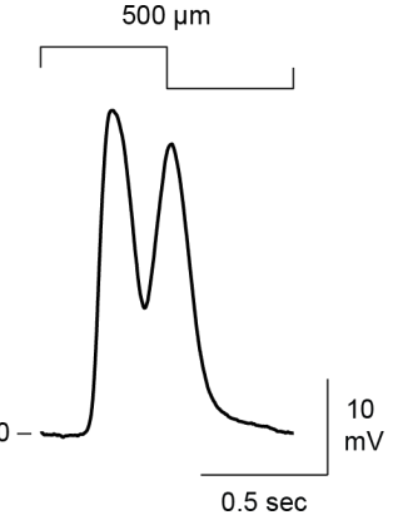

B

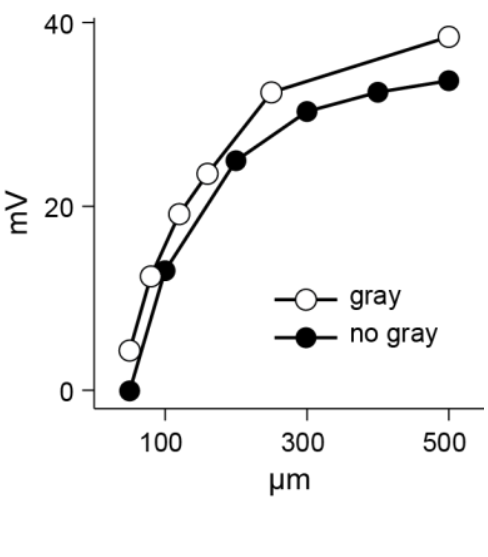

Figure 4.4 Receptive field estimation with spots without gray preframes. A. Response traces of a recorded bipolar cell to spots of different diameters that switched between black and white, thus without intermediate gray screen.

B. Comparison of the tuning curve with gray intermediate screen (black line with black dots) and without gray intermediate screen (black line with white dots). The continuous increase can be observed in both types of stimuli.

\subsubsection{Center estimation with binary white noise}

In visual neuroscience, binary white noise commonly refers to a stimulus, where the screen is subdivided into squares that randomly change between two contrast values. We subdivided our screen into squares of $\sim 20-30 \mu \mathrm{m}$ and we changed the contrast inside a square randomly to either black or white every $\sim 33 \mathrm{~ms}$ (for some cells we used $40 \mathrm{~ms}$ or $66 \mathrm{~ms}$, see methods 3.5). To estimate the receptive field center, the weighted average over all stimulus 
signals, preceding every membrane potential change, is built (see methods 3.5.2). This reverse correlation method provides a description of the receptive field of a cell and identifies the location and size of its receptive field.

The receptive fields of three bipolar cells measured with binary white noise are shown in Figure 4.5. The response polarity is reflected in the contrast of the squares i.e. ON cells respond to white squares, OFF cells to black squares. All cells had the same response polarity as in the previously introduced full-field light steps and spots. Further, we found cells with larger receptive fields (Fig.4.5 A \& C) and cells with smaller receptive fields (Fig.4.5B). The population distribution of the measured center sizes ranged between 30 to $210 \mu \mathrm{m}(2$ sigma, $n=31)$ and is shown in Figure 4.5D. 84\% of the cells had diameters between 50-150 $\mu \mathrm{m}$. The distribution is in line with previous estimations with binary white noise, which reported salamander bipolar cell diameters in the range of 35-120 $\mu \mathrm{m}$ (Ölveczky et al., 2003) and 50-150 $\mu \mathrm{m}$ (Baccus et al., 2008).

A

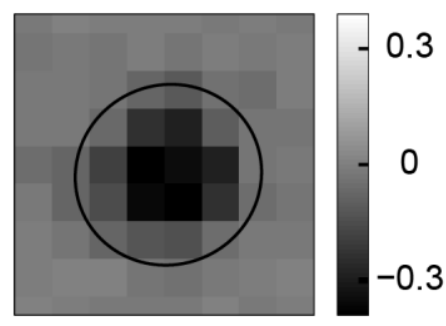

C

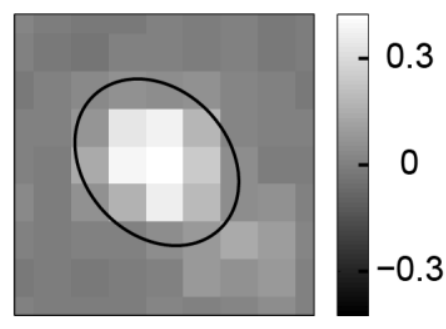

B
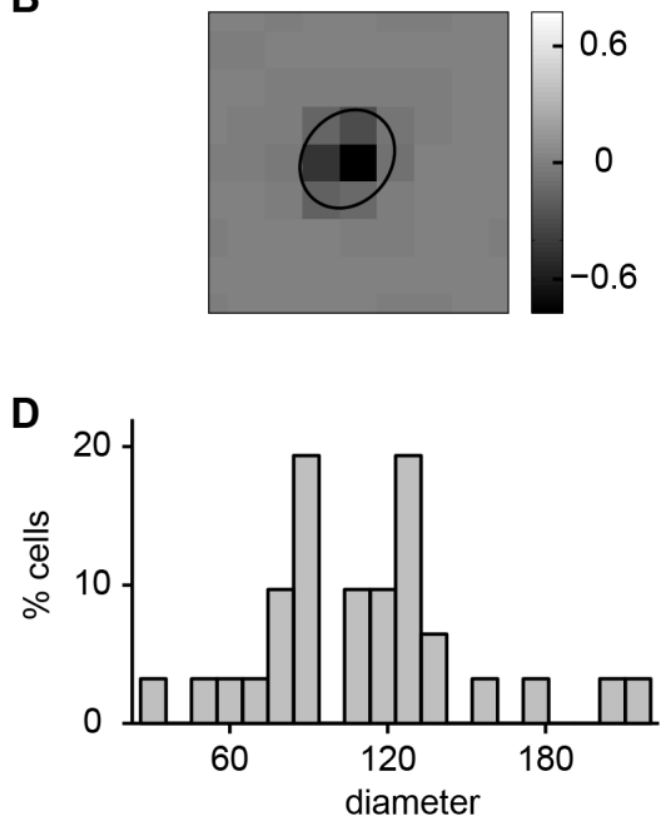

Figure 4.5. Receptive field estimation with white noise. Three examples cells for receptive field center estimation with binary white noise and population distribution. A \& B. Show the receptive field center estimation of two OFF bipolar cells. The receptive field of the bipolar cell in A has a larger diameter of around $150 \mu \mathrm{m}$. The receptive field diameter of the bipolar cell in B is smaller with a diameter of around $80 \mu \mathrm{m}$. C shows the receptive field center of an ON cell with a diameter of around $130 \mu \mathrm{m}$. D. Population distribution of the estimated center diameters with binary white noise ( 2 sigma, $\mathrm{n}=31)$. 


\subsubsection{Center estimation: spot vs. binary white noise}

We compared the estimated center sizes of the spot stimulus to the estimated center sizes of the binary white noise stimulus. The two were strongly correlated, the larger the diameter was estimated with spots, the larger it was estimated with binary white noise (Fig.4.6A, $r=0.68$, $p=0.002, \mathrm{n}=18)$. However, the measured sizes were significantly different from each other (Fig.4.6B, $p=0.001, \mathrm{n}=18)$. Most of the cells $(\sim 95 \%)$ have larger diameters when measured with spots compared to the binary white noise estimation. This discrepancy between the diameters was also found when studying the literature. Fahey and Burkhardt (2003) for example reported diameter sizes of $272-515 \mu \mathrm{m}$ with spots; these are much larger than the diameter sizes of 35-120 $\mu \mathrm{m}$ measured with binary white noise by Ölveczky et al. (2003). To see such large diameter sizes with spot stimuli in salamander bipolar cells is surprising given their dendritic field sizes of $\sim 25$ $175 \mu \mathrm{m}$ (Wu et al., 2000) and other studies even reported smaller sizes of $\sim 43-70 \mu \mathrm{m}$ (Borges and Wilson, 1987). The binary white noise receptive fields of $30-210 \mu \mathrm{m}$ are more comparable to those dendritic field sizes of $25-175 \mu \mathrm{m}$. Thus, it might be that the binary white noise stimulus captures the center size more accurate.

As already indicated, a reason for the larger receptive fields with spots could be poor centering of the spot over the "real" position of the cell. For the spots, the location of the cell was determined manually by moving a flashing spot around the retina (see methods 3.5.2). For some cells we tested whether the manually determined spot receptive field location differ from with the binary white noise receptive field locations. We did not find a significant difference between the locations and further, there was no correlation between the difference in the location and the estimated diameter size of the spot $(\mathrm{r}=-0.16, p=0.7)$. Thus, there might be other explanations for the large diameter sizes with spots that we investigate in the discussion of this thesis.

Taken together, the receptive field sizes measured with spots varied between $80-1000 \mu \mathrm{m}$ and the receptive field sizes measured with binary white noise between 30-210 $\mu \mathrm{m}$. For further analysis, we chose the receptive field size measured with binary white noise and refer to it as the diameter of the cell. 
A

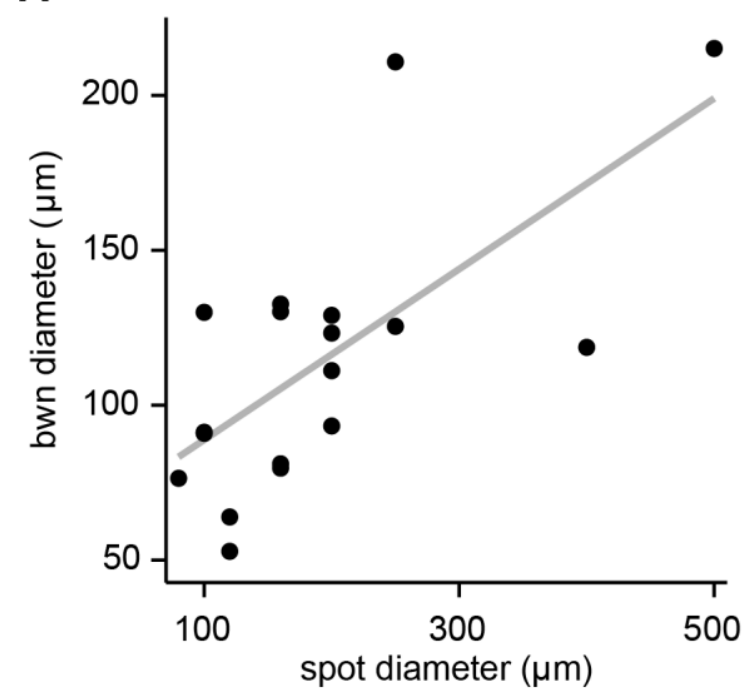

B

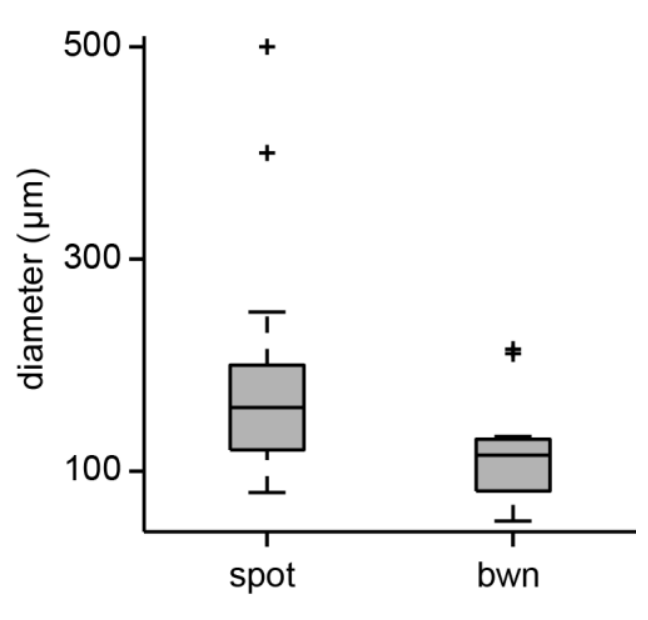

Figure 4.6. Comparison of receptive field sizes measured with spots vs. binary white noise. A. Diameter sizes measured with spots are plotted against the diameter sizes measured with binary white noise (bwn). The estimated diameters with the two methods are significantly correlated $(\mathrm{r}=0.68, p=0.002, \mathrm{n}=18)$. B. The receptive field diameters measured with spots are significantly larger than the diameters estimated with binary white noise (bwn) stimulus $(p=0.001, \mathrm{n}=18)$. Note, for the reported analysis the cells with a spot diameter above 500 $\mu \mathrm{m}$ were excluded.

\subsection{Receptive field surround estimation}

A classical way to measure the surround antagonism is by comparing the response to a spot of optimal diameter, i.e. whose diameter matches the receptive field center, with the response to a large spot i.e. whose diameter exceeds the receptive field center (Fahey and Burkhardt, 2003; Hare and Owen, 1990; Krieger et al., 2017). If the response to the large spot, compared to the center spot, is reduced, it is evidence for an antagonistic i.e. inhibitory surround. Figure 4.7A shows superimposed responses of an ON bipolar cell to a center spot of $160 \mu \mathrm{m}$ and a large spot of $1000 \mu \mathrm{m}$. The peak response to the large spot was reduced by about $36 \%$ compared to the peak of the center spot. Figure 4.7B shows the responses of an OFF bipolar cell without an inhibitory surround. Here, the center response is very similar to the large spot response. Over the 23 bipolar cells, we found response reductions between $0 \%$ - 70\% (Fig.4.7C). Around $40 \%$ of our recorded cells showed a reduction in the response of more than $10 \%$, while the remaining $60 \%$ did not show an inhibitory surround effect.

We compared the surround index to the diameter size as well as the response polarity. We did not find a relationship of the surround index to either the size of the diameter or the response polarity. 
A

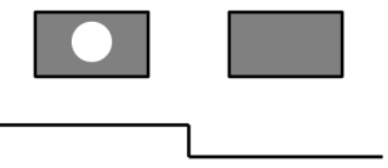

surround strength $=\sim 36 \%$

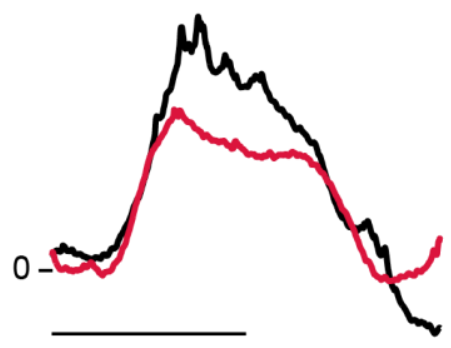

B

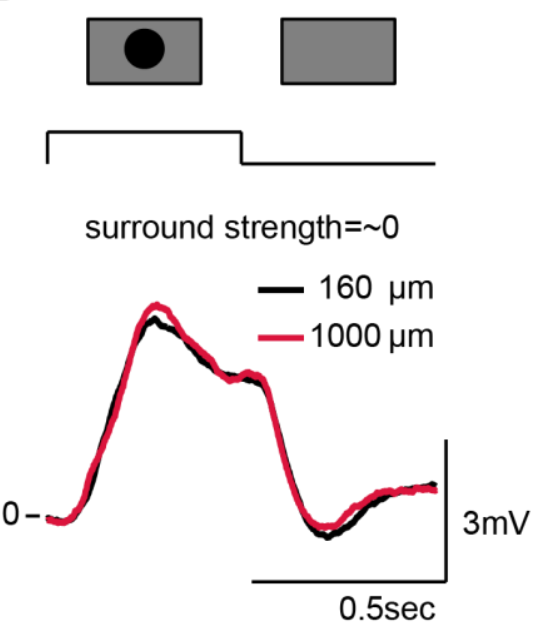

C

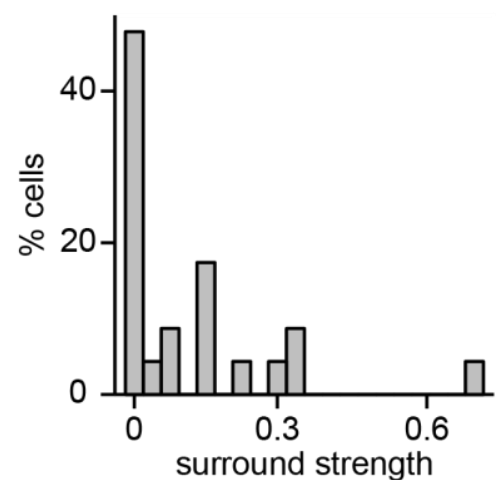

Figure 4.7. Receptive field surround estimation. A. Response traces of a recorded ON bipolar cell to the optimal center spot $(160 \mu \mathrm{m}$, black trace) and to the large spot $(1000 \mu \mathrm{m}$, red trace). The recorded ON bipolar cell reduced its amplitude to a large spot by about 37\%. B. Example of an OFF bipolar cell with similar amplitude response traces to the optimal and large spot. C. Population distribution of the estimated surround strength ranging between $0-70 \%$ reduction of the amplitude to the large spot relative to the center $\operatorname{spot}(\mathrm{n}=23)$.

\section{Excurse: Gray or no gray?}

As for the receptive field center estimation, 9 out of the 23 recorded cells in Figure 4.7C, were recorded with spots that switched between black and white. For 7 cells we recorded both stimuli and tested whether the different stimulus designs had an effect on the surround estimation. We did not find a significant difference between the surround strength estimated with the spot with gray screen and the black-white switching spot $(p=0.56, \mathrm{n}=7)$. As for the center estimation, we excluded the possibility that the intermediate gray screen can change the surround strength.

\subsection{Temporal properties}

In the previous chapters, we looked at the response polarity and center-surround structure of the recorded bipolar cells. In the current subchapter, we inspect their response profiles. Traditionally, the response profiles are analyzed with respect to the temporal properties of a cell. The classical temporal properties are the duration of the response ("transient" vs. "sustained") and the latency of the response ("fast" vs. "slow"). It has been shown that the temporal properties of bipolar cells are different under a center and full-field stimulation (Fahey 
and Burkhardt, 2003; Franke et al., 2017). Therefore, we examined the temporal properties first under full-field light stimulation (section 4.4.1), then under center spot stimulation (section 4.4.2) and finally we compared the findings (section 4.4.3).

\subsubsection{Temporal properties under full-field light steps}

\section{Response duration}

Based on the response duration, bipolar cells are traditionally subdivided into transient and sustained cells. As shown in Figure 4.8, we observed sustained (A) and transient (B) bipolar cells under the light step of the preferred contrast polarity. Further, as described in more recent studies (Franke et al., 2017), we also observed cells with an initial peak that is followed by a sustained plateau response (Fig.4.8C). We quantify the duration of the response by computing a sustained-transient index (STi, see methods 3.5.3). Values close to 1 stand for sustained responses, while values close to 0 stand for transient responses (see the corresponding STi values for the example cells in Figure 4.8A-C). The population distribution of the sustained-transient index (STi) is shown in Figure 4.8D. The responses ranged from sustained membrane potential depolarization for more than 1 second, to transient membrane potential changes. Similar to analysis performed in retinal ganglion cells (Carcieri et al., 2003), we tested whether bipolar cells can be grouped into two distinct classes of "transient" vs. "sustained" cells. We found that the bipolar cells could not be subdivided into two distinct groups (transient vs. sustained), the distribution of the sustained-transient index was unimodal (bimodality was rejected, $p=0.8$, $\mathrm{n}=40$ ). From our data, it seems that the response duration of bipolar cells follows a continuum with transient and sustained responses on the extremes and more slowly or fast decaying cells in the middle. 
A

sustained cell average response
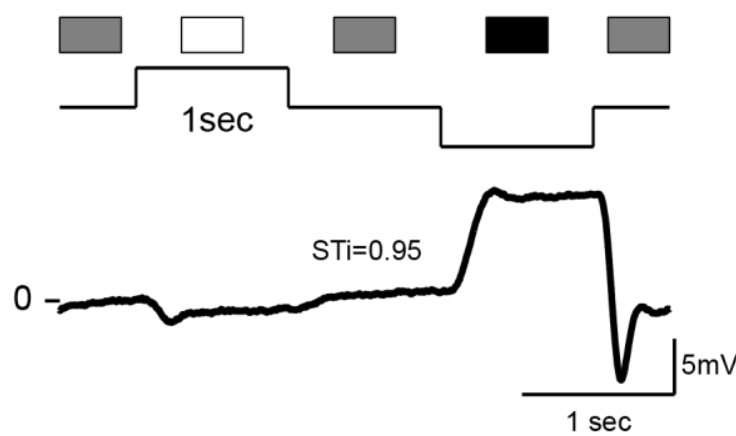

single trial response

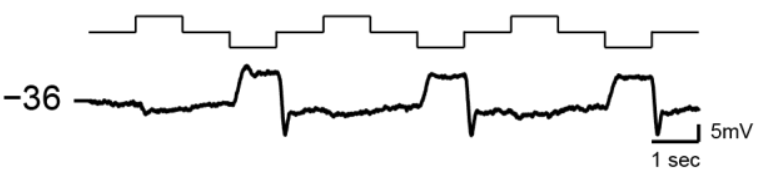

C

plateau cell average response

0

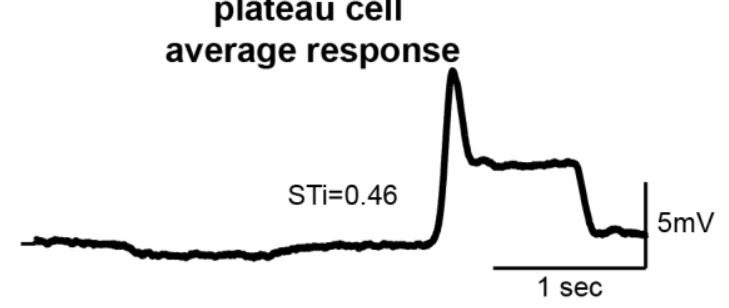

single trial response

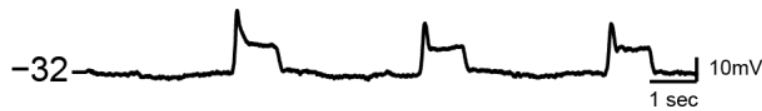

B

transient cell average response

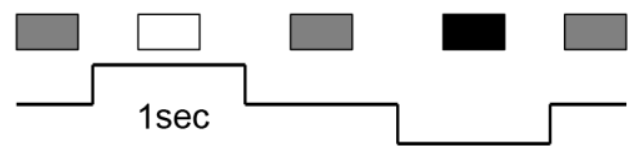

0

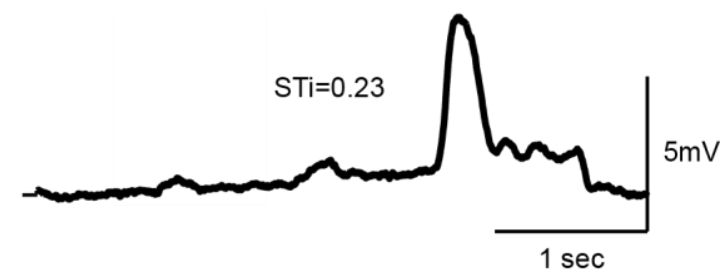
single trial response

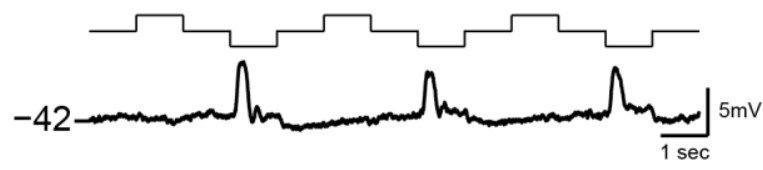

D

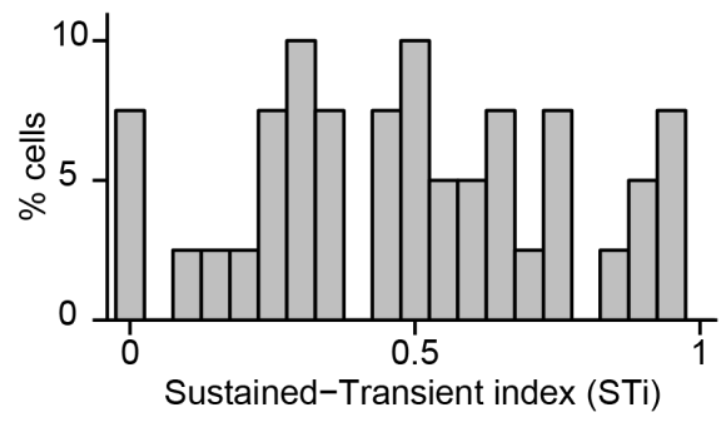

Figure 4.8. Response duration. Example traces to the full-field light steps and population distribution for the response duration. A-C. Shows the average and single trial responses of three OFF bipolar cells. We found sustained responses (A), transient responses (B) and bipolar cells that settled on a steady plateau after the first peak (C). D. Population distribution of the sustained-transient index (STi). The distribution was found to be unimodal (bimodality was rejected, $p=0.8, \mathrm{n}=40$ ).

\section{Oscillations}

Curiously, we discovered OFF bipolar cells that showed oscillations in their response profiles (example cell in Fig.4.9A). The example cell responded to the light decrement with a $\sim 4 \mathrm{~Hz}$ cycle (see methods 3.5.3 for how the oscillation frequency is determined). There is evidence for slow oscillations at around $4 \mathrm{~Hz}$ in OFF dogfish bipolar cells (Ashmore and Falk, 1980) and at around 5-10Hz in goldfish ON bipolar cells (Burrone and Lagnado, 1997).

However, these oscillations are rarely reported and their role remains unclear. In Figure 4.9B, we 
show the population distribution of the oscillation frequency. For $55 \%$ of the cells, this frequency is at around $1 \mathrm{~Hz}$, meaning they did not show oscillations in their response profiles but simply responded to the light step with one cycle. For around $45 \%$ of the cells however we found oscillation frequencies between $2-6 \mathrm{~Hz}$, meaning they responded with more than one response cycle to the stimulus. Furthermore, the population distribution was found to be bimodal $(p=0.003, \mathrm{n}=40)$. Therefore, in some of our following analysis, we subdivided our bipolar cells into two groups: 1. non-oscillatory group (frequencies between $0-1 \mathrm{~Hz}$ ) 2. oscillatory group (frequencies 2-6Hz).

A
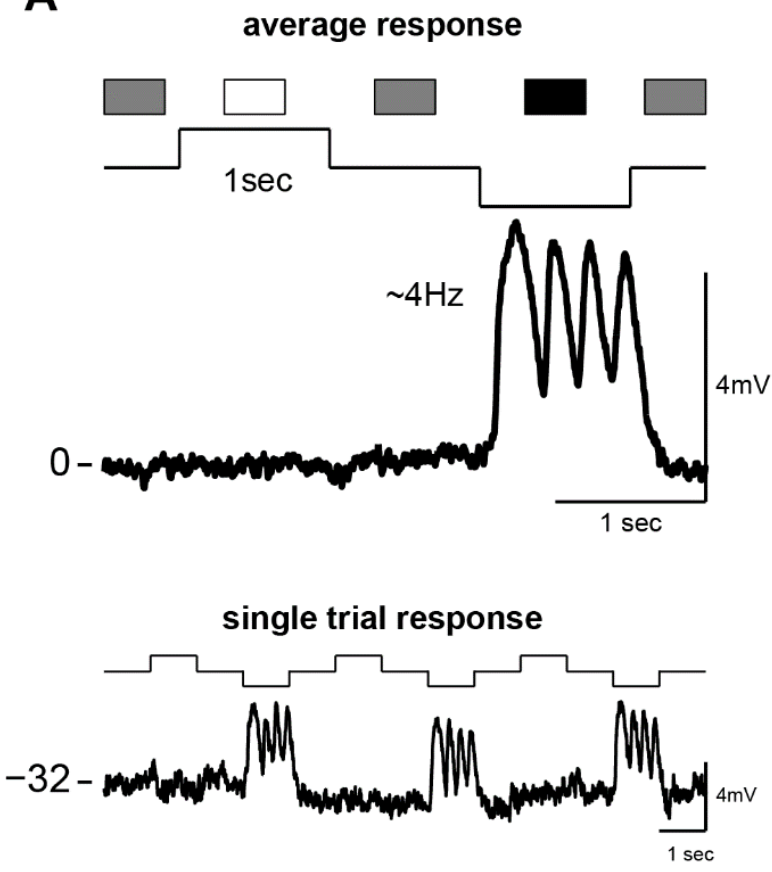

B

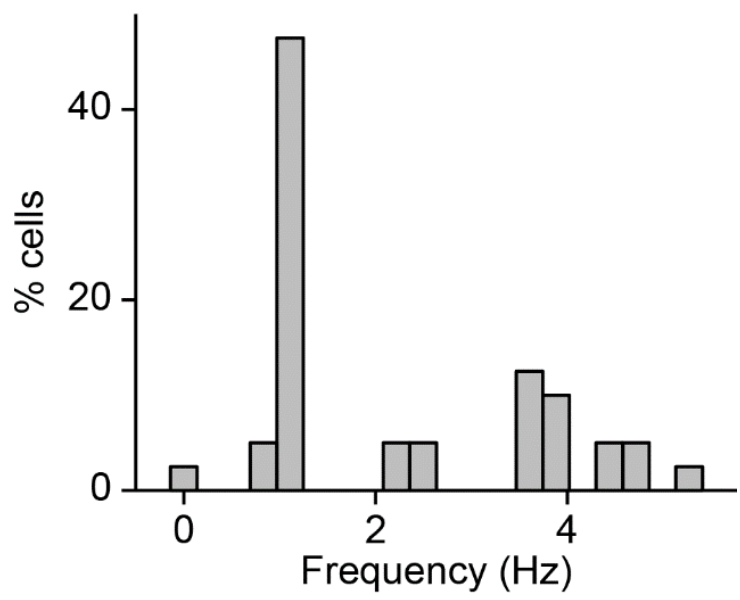

Figure 4.9. Oscillations in the responses of bipolar cells. Example of an oscillatory response to the full-field light steps and population distribution of the oscillation frequencies. A. Shows the average and single trial responses of an OFF bipolar cell. The bipolar cell responded to the light decrement with a $\sim 4 \mathrm{~Hz}$ cycle (visible both in the single trials as well as in the average response). B. Population distribution of the oscillation frequency $(n=40)$. For around $45 \%$ of the cells we found oscillations between $2-6 \mathrm{~Hz}$.

\section{Response latency}

A further temporal property is the latency of the response ("fast" vs. "slow"). A way to measure the latency is by building the time difference between the onset of the light step (dashed line in Fig.4.10A) and the first detectable deviation of the response from the baseline (red point Fig.4.10A). Figure 4.10B shows superimposed responses of different OFF bipolar cells to a light decrement from gray to black. Here, the cell 1 (green response trace) exhibit longer latencies compared to cell 2 (black response trace). The population distribution of the latency measured in 
$\mathrm{ms}$ is shown in Figure 4.10C (range 44-230 ms). The smaller the latency values, the faster the cells responded to the onset of the stimulus. The bipolar cells could not be subdivided into two groups (fast vs. slow), the distribution of the response latency was found to be unimodal (bimodality was rejected, $p=0.65, \mathrm{n}=40$ ). Again, it seems that the response latency of bipolar cells follows a continuum with fast and slow responses on the extremes and intermediate latencies in the middle.

A

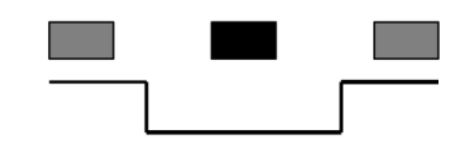

$1 \mathrm{sec}$

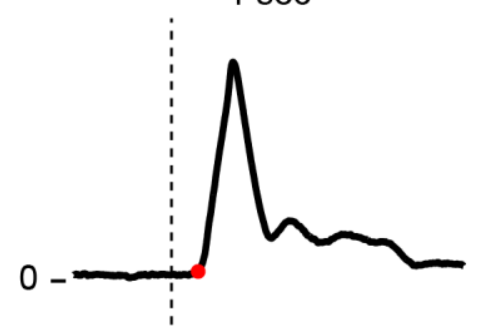

B

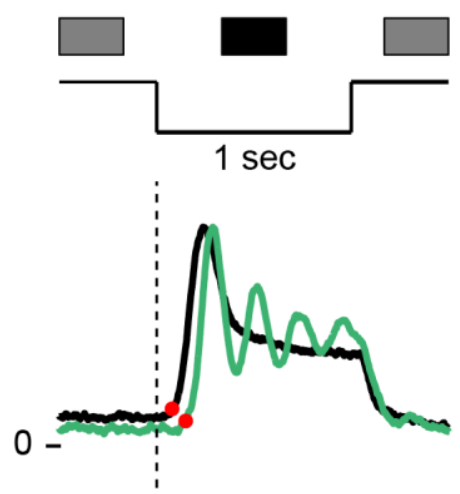

C

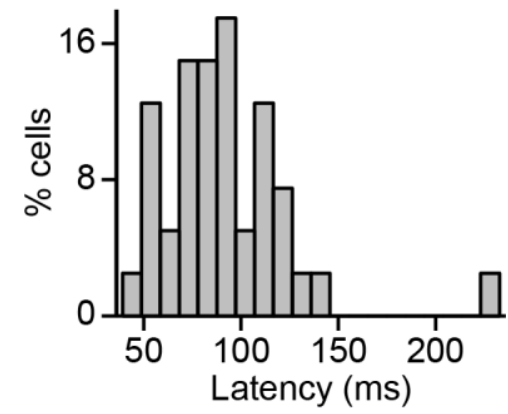

Figure 4.10. Response latency. Example of responses to the preferred light steps and population distribution for the response latency. A. The response latency is measured as the difference in time between the onset of the preferred stimulus (here black screen, dashed line) and the first detectable deviation of the response from the baseline (red dot). B. Shows the average responses of two OFF bipolar cells. We found slower responses (green response trace) and faster responses (black response trace). C. Population distribution of the latency, measured in ms. The distribution was found to be unimodal (bimodality was rejected, $p=0.65$, $n=40)$.

Finally, we also compared whether the described properties were correlated among each other. We did not find a correlation between the response onset (fast vs. slow) and the duration of the response (sustained vs. transient). We also did not observe a connection to the presence of oscillations. Furthermore, none of the temporal properties was correlated to the receptive field diameter. Due to the small amount of ON cells $(n=4)$, we did not study whether the temporal properties are different between the ON and OFF cells.

\section{Excurse: Gray or no gray?}

The 11 cells recorded with light steps that switched directly between black and white, thus without an intermediate gray screen, were excluded from the analysis of the temporal properties. We discovered that the gray screen had an influence on the temporal properties. The cells 
recorded without intermediate gray light steps responded much slower to the stimulus $(p<0.01$, $\mathrm{n}=11)$.

\subsubsection{Temporal properties with center spots}

In the previous section, we described the responses of bipolar cells to full-field light steps. Here, the measured response in a cell is composed of center and surround activation together. In a next step, we estimated the temporal properties of the center only. To do so, we analyzed the response traces to the spot size that matches the receptive field size of the cell. Similar to the fullfield stimulation, we observed sustained, transient and plateau-like-responses (see Fig.4.11A-C). Further, we observed responses with different latencies and responses showing oscillations (Fig.4.11D \& E).

A
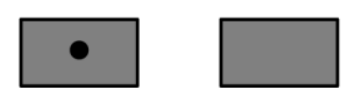

$0.5 \mathrm{sec}$

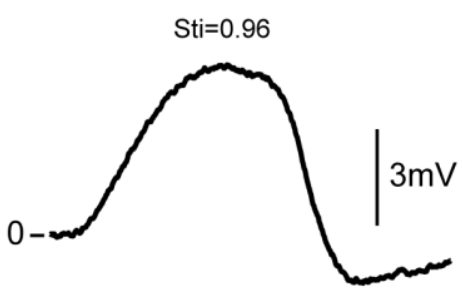

B

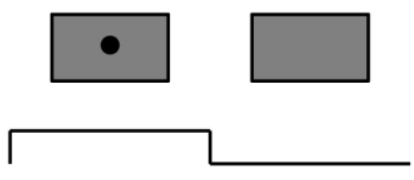

Sti $=0.21$

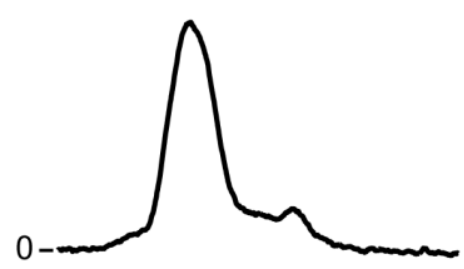

C
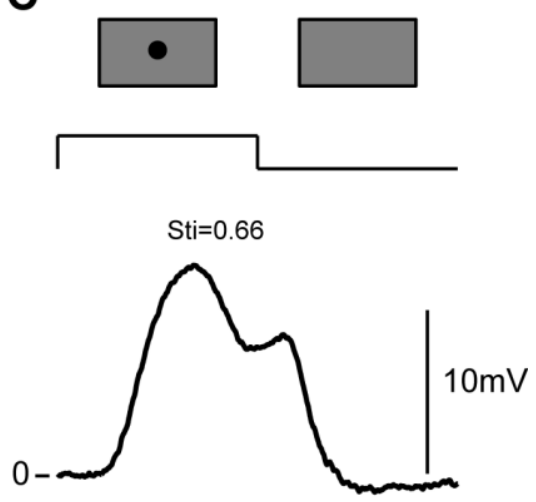

D

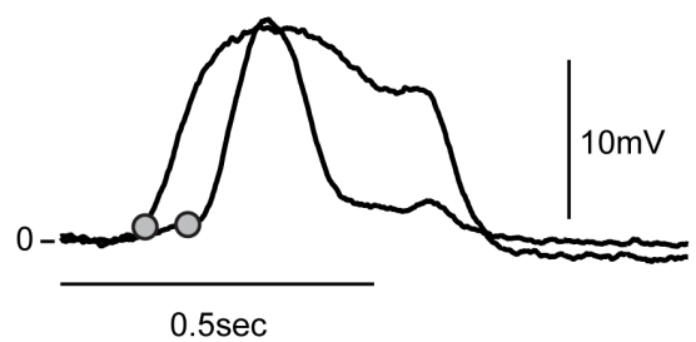

E

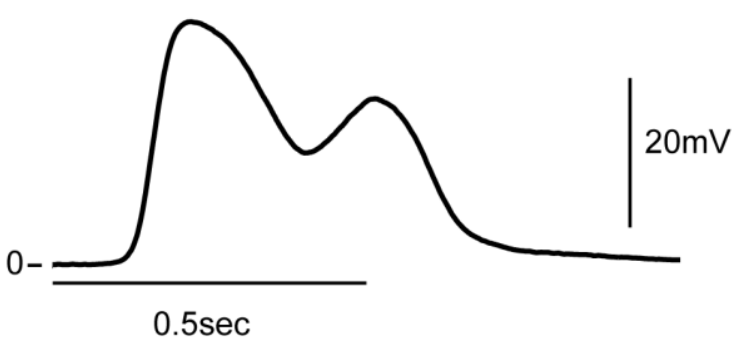

Figure 4.11. Temporal properties under a center spot stimulation. A. The response trace of a sustained bipolar cell to a center spot with a sustained-transient index (STi) of $\sim 1$. B. The response trace of a transient bipolar cell $(\mathrm{STi}=\sim 0.2)$. C. The response trace of a bipolar cell with a steady plateau response after the first peak (STi= 0.65). D. The response traces of two different bipolar cells with different latencies to a center. E. Example bipolar cell with oscillations during a receptive field center stimulation. 
The population distribution of the sustained-transient index (STi), latency and oscillation frequency to the center light stimulation are shown in Figure 4.12A-C. We did not find a correlation among the different central temporal properties as well as with the receptive field diameter or surround strength. Further, due to the low number of cells $(n=11)$, we did not test for bimodality in our distribution.

Note, the quantification of the oscillation frequency was more difficult during the center light stimulation. Here, we only presented the spot for 0.5 seconds, thus the frequency resolution is lower compared to the full-field light stimulation of 1 second (see also methods 3.5.3). The observed peak at $2 \mathrm{~Hz}$ ( $80 \%$ of the cells) corresponds roughly to the peak found at $\sim 1.5 \mathrm{~Hz}$ during the full-field light stimulation. The frequency of $\sim 4 \mathrm{~Hz}$ corresponds roughly to a frequency of $\sim 3-5 \mathrm{~Hz}$ under a 1 second stimulation.

A

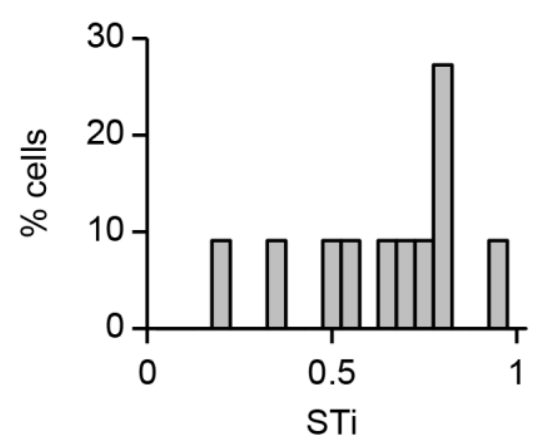

B

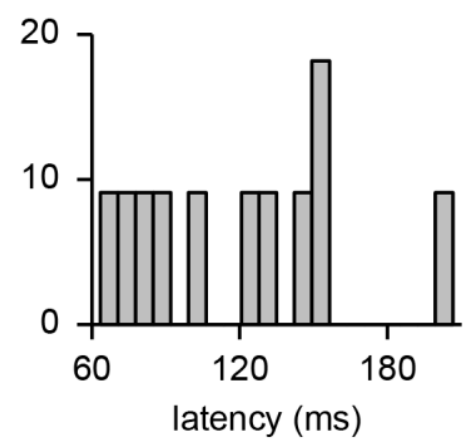

C

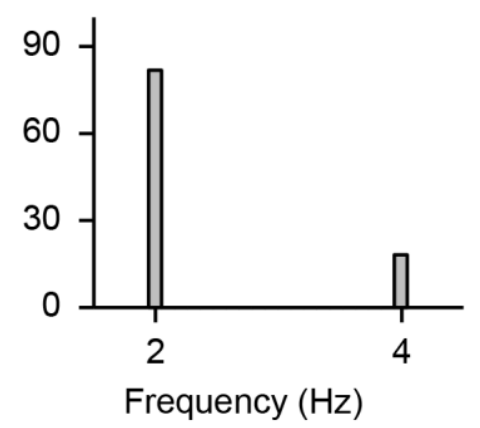

Figure 4.12. Population distributions of the temporal properties to a center spot stimulation. A. Population distribution for the sustained-transient index (STi), range between 0.21-0.96. B. Population distribution for the latency in ms, range between 67-203ms. C. Population distribution for the oscillatory frequency, range between 24Hz. $(\mathrm{n}=11)$

\section{Excurse: Gray or no gray?}

The 9 cells recorded with spots that switched directly between black and white, thus without an intermediate gray screen, were excluded from the analysis of the temporal properties of the center. Similar to the full-field stimulation, we discovered that the gray screen had an influence on the temporal properties. For seven cells, we recorded both stimuli and therefore tested whether the different stimulus designs had an effect on the temporal properties of the same cell. The cells responded much slower to a black-white spot compared to a spot with gray screen $(p<0.01, \mathrm{n}=7)$. 


\subsubsection{Are the temporal properties different between center and full- field stimulation?}

Recently, it has been shown, that the temporal properties change under a center and fullfield stimulation (Franke et al., 2017). Therefore, we compared the temporal properties of the receptive field center with the temporal properties under the full-field light steps. In Figure 4.13A, we overlaid the response traces during center and full-field stimulation for three example cells. The step duration of the full-field light stimulation was 1 second, while for the spot we used 0.5 seconds. To compare the response durations of the two stimuli, we therefore only looked at the first 0.5 seconds of the full-field light stimulation (see methods 3.5.3). As one can see from the example cells, the response duration was similar under center and full-field light stimulation. Cell 1 for example, showed similar sustained response profiles, cell 2 responded transiently to both center and full-field stimulation. Overall, we observed a clear correlation between the sustained-transient index (STi) under a center and full-field stimulation $(r=0.94, p<0.001, n=9$, Fig.4.13B). Due to the different step durations between full-field and center stimulation (1 sec vs. $0.5 \mathrm{sec}$ ), we also compared the sustained-transient index (STi) between the largest spot (gray trace) and the center spot. Both spots are recorded with a duration of 0.5 seconds and the largest spot of $1 \mathrm{~mm}$ is covering the screen almost like a full-field stimulation. We found a similar strong correlation between the center and large spot $(p=0.94, p<0.001, \mathrm{n}=14)$.

Curiously, we observed a different picture for the response latency. Here, we did not observe a correlation between the center and full-field stimulation ( $\mathrm{r}=0.34, p=0.36, \mathrm{n}=9$, Fig.4.13C). As one can see from the single examples, some cells responded faster during center stimulation (e.g. cell 1), other cells responded more slowly (e.g. cell 2) and some cells did not change their latencies between center and full-field stimulation (cell 3). This picture was almost identical when comparing the latencies of the center with the latencies of the large spot $(r=0.30$, $p=0.29, \mathrm{n}=14)$. 
A

Cell 1
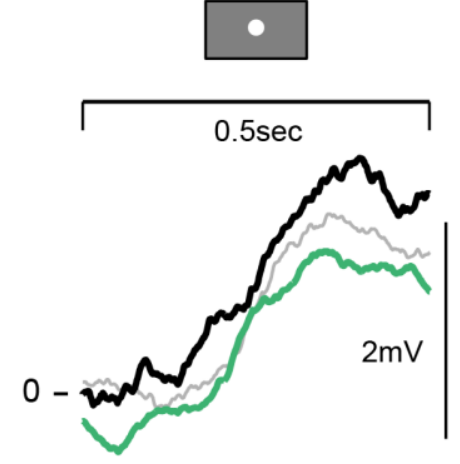

Cell 2
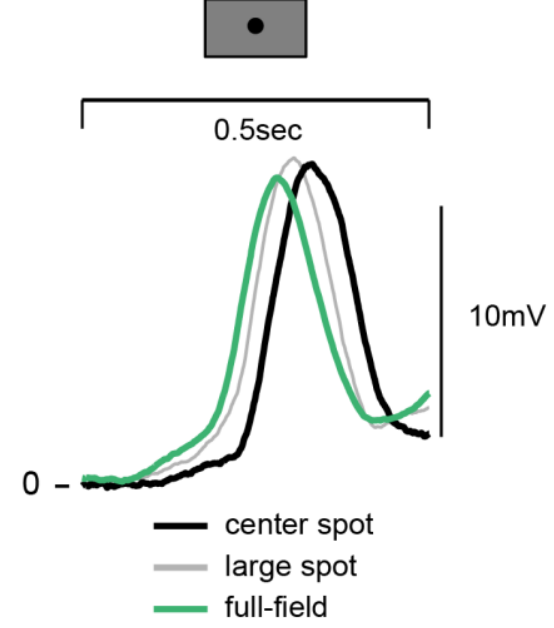

Cell 3

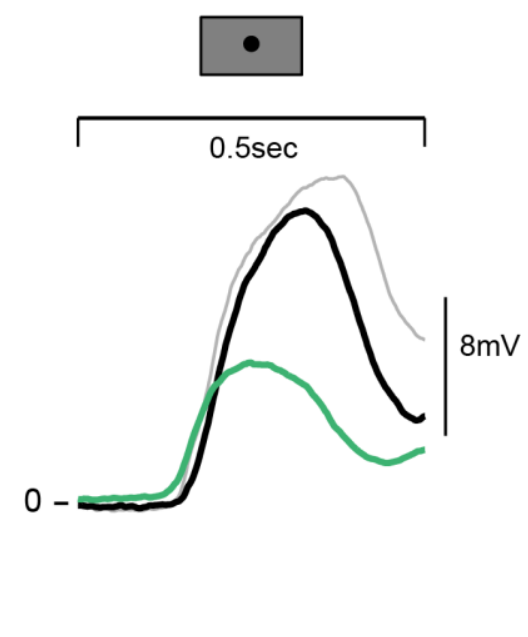

B

Sustained-transient index (STi)

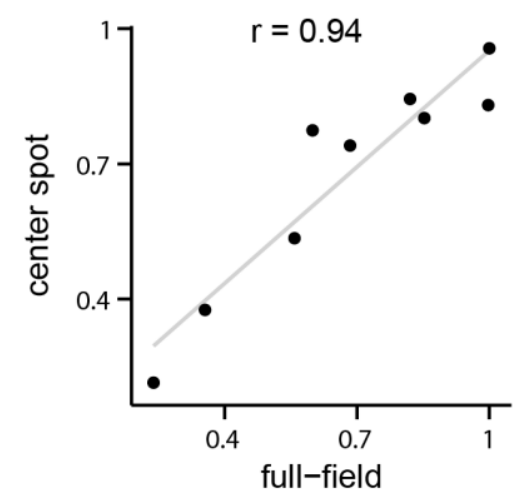

C Latency (ms)

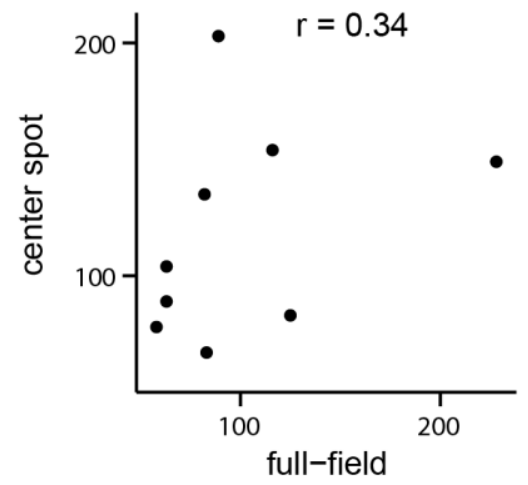

Figure 4.13. Comparison of the temporal properties under center and surround stimulation. A. Three example cells with response traces under a center spot (black), large spot (gray) and full-field (green) stimulation. B. The sustained-transient index (STi) under center and full-field stimulation showed a clear correlation $(\mathrm{r}=0.94$, $p<0.001, \mathrm{n}=9)$. C. The latency under center and full-field stimulation showed no relationship ( $\mathrm{r}=0.34$, $p=0.36, \mathrm{n}=9)$.

For the oscillatory responses, we found that most cells that showed oscillations during the full-field stimulation also oscillated under a center stimulation. For some cells the frequency of the oscillation was similar under center and surround stimulation (e.g. Fig.4.14A, 4Hz for both type of stimulations). For other cells, the frequency under a full-field stimulation was higher (e.g., Fig.4.14B, frequency at the center was $4 \mathrm{~Hz}$ and full-field 5Hz). A direct comparison of the oscillatory frequencies has to be interpreted with caution, because the duration of the spot was shorter and therefore the frequency resolution with the spot was lower $(\mathrm{r}=0.6, p=0.09, \mathrm{n}=9)$. 
A

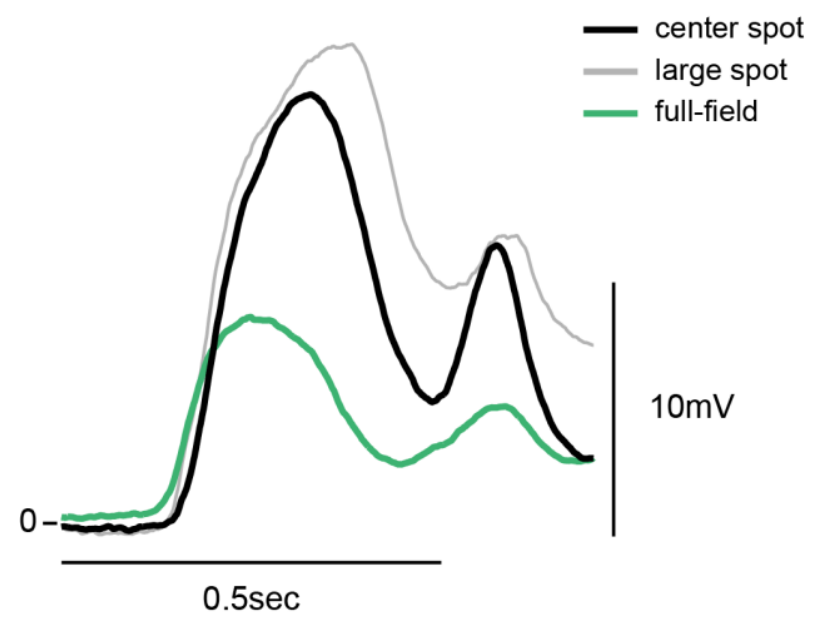

B

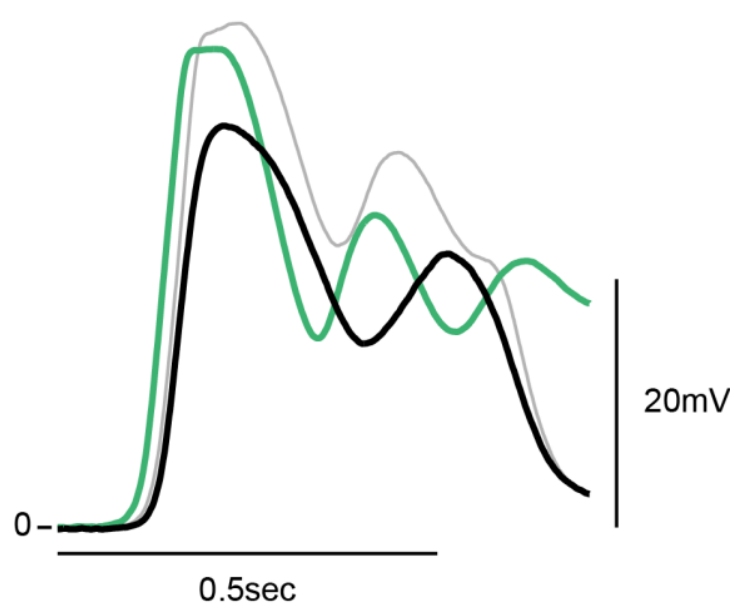

Figure 4.14. Comparison of oscillatory responses under center and surround stimulation. A. Example of a bipolar cell that showed a $4 \mathrm{~Hz}$ oscillation to center (black), large spot (gray) and full-field (green) stimulation. B Example of bipolar cell that showed a $4 \mathrm{~Hz}$ oscillation to the center spot and a $5 \mathrm{~Hz}$ oscillation to the full-field stimulation.

Overall, we concluded that the response duration, i.e. how transient or sustained the cell responded, was similar under center and full-field stimulation and therefore less influenced by a surround. Curiously, the response latency, i.e. how fast or slow the cell responded, was different under center and full-field stimulation and therefore influenced by the surround. Further, our results indicate that bipolar cells also oscillate under a center stimulation, whether however, the surround can influence the speed of the oscillations remains an open question.

\section{Are the temporal properties connected to the white noise stimulation?}

From the binary white noise stimulus, we can estimate the spatiotemporal filter of the cell. The spatial component of the filter is used to estimate the receptive field center (section 4.2.2); the temporal component of the filter (see methods 3.5.3) contains information about the temporal properties of the cell like for example the latency or duration (Fig.4.15). For retinal ganglion cells, the temporal filters are often used to characterize the temporal dynamics of their responses (Segev et al., 2006). The time course from the binary white noise stimulus is usually interpreted as a measure for the temporal properties in the receptive field center of the cell (Chichilnisky, 2001).We tested this idea by comparing the temporal properties during center spot stimulation with the binary white noise stimulation. Surprisingly, the latencies of the center spot stimulus were not correlated to the latencies of the binary white noise time course $(r=0.23$, 
$p=0.55 \mathrm{n}=9$ ). However, the latencies of the full-field stimulation were significantly correlated with the latencies of the binary white noise stimulation $(\mathrm{r}=0.47, p=0.02, \mathrm{n}=26)$.

The biphasicness of the temporal filter is interpreted as a measure for how transient a cell is. Yet, we did not find a correlation between the degree of biphasicness and the duration of the response for the center spot $(\mathrm{r}=0.21, p=0.59, \mathrm{n}=9)$ as well as for the full field steps $(\mathrm{r}=0.11$, $p=0.58, \mathrm{n}=26$ ). We found bipolar cells with a biphasic filter and a sustained response, further, we found bipolar cells with a monophasic filter and a transient response (Fig.4.15).

To conclude, for all our further analysis, we used the temporal properties of the spot stimulus as a measure for the properties in the receptive field center. The temporal properties of the surround are measured with the full-field light steps.

\section{cell 1}

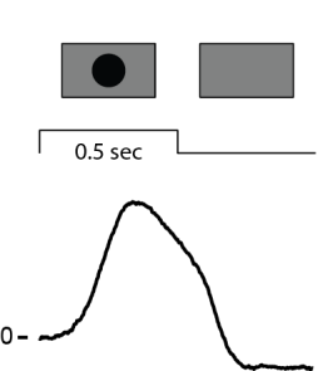

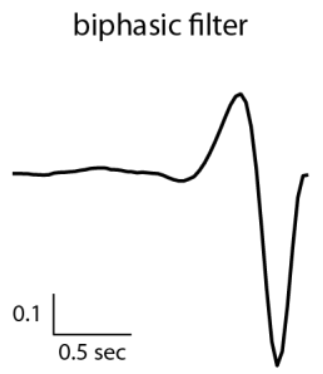

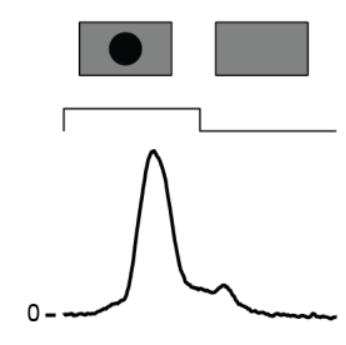

\section{cell 2}

monophasic filter

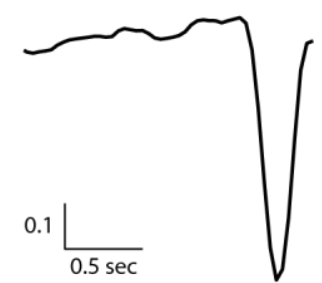

Figure 4.15. Comparison of the response duration between white noise and spots. Cell 1 showed a sustained response to a spot covering the receptive field center and a biphasic filter. Cell 2 showed a transient response to a center spot and a monophasic filter. 



\section{Nonlinearities in bipolar}

\section{cells}

With the general response characteristics in our pockets, we are ready to investigate the second goal of this thesis. It has been proposed that at the heart of the response diversity observed in ganglion cells lie nonlinear signal transformations in bipolar cells (Gollisch, 2013; Gollisch and Meister, 2010; Roska and Meister, 2014). In our goal 2, we aimed to describe how these nonlinear signal transformations manifest themselves in bipolar cells. Nonlinear signal transformation can occur at two stages in bipolar cells: at their output and their inputs. Thus, we studied whether bipolar cells transform light linearly or nonlinearly by measuring their output response function (subchapter 5.1). Furthermore, we investigate whether bipolar cells integrate their inputs linearly or nonlinearly by measuring their spatial integration properties (subchapter $5.2)$.

\subsection{Nonlinearities in the output response function}

The output response function describes how neurons respond to an input light stimulus with various intensity. A linear output function means that the neuronal response changes proportional to the input light stimulus. For example, when the strength of the input is doubled, the cell doubles the magnitude of its response. Various studies measuring the output function at the bipolar cells' somas proposed a linear relationship (Baccus and Meister, 2002; Rieke, 2001; Sakai and Naka, 1987a; Toyoda, 1974). Yet, other studies reported nonlinear responses 
(Burkhardt and Fahey, 1998; Fahey and Burkhardt, 2003). The inconsistency between the different studies has been attributed to different stimulus dynamics and bipolar cell types (Burkhardt and Fahey, 1998; Schwartz and Rieke, 2011). For example, Burkhardt and Fahey (1998) showed spots, while Rieke (2001) showed a full-field white-noise contrast sequence. Thus, we measured the output function with both types of stimuli: spot stimuli (section 5.1.1) and white noise (section 5.1.2) and compared the results (section 5.1.3). Further, we investigated whether bipolar cells with different general response characteristics (e.g. sustained vs. transient) showed different nonlinear signal transformation (section 5.1.4).

\subsubsection{Nonlinearities measured with spots}

The spots used to measure the receptive field center-surround structure, were shown once with black contrast ( $-100 \%$ contrast) and once with white contrast ( $+100 \%$ contrast). This stimulus design allowed us to investigate for each spot diameter size, the response to the preferred (e.g. black) and non-preferred (e.g. white) contrast. A linear bipolar cell is expected to show a similar amount of hyper- and depolarization to the preferred and non-preferred spot. A nonlinear bipolar cell is expected to show a different amount of hyper- and depolarization to the preferred and non-preferred spot. We indeed found bipolar cells that showed such an equal amount of depolarization and hyperpolarization (see example cell in Fig.5.1A). However, almost 90\% of our recorded bipolar cells showed unequal amounts of hyper- and depolarization i.e. nonlinear responses. Thereby, they varied in their imbalance between hyper- and depolarization. For example, the bipolar cell in Figure 5.1B showed a depolarization to the preferred stimulus of $\sim 5 \mathrm{mV}$, while the hyperpolarization was $\sim 2 \mathrm{mV}$ or in other words $\sim 40 \%$ of the depolarization. The response of the bipolar cell in Figure 5.1C was even more nonlinear; the cell strongly depolarized to the preferred stimulus $(\sim 20 \mathrm{mV})$ but did not show hyperpolarization to the nonpreferred stimulus (rectification). We quantified the degree of hyper- and depolarization imbalance by computing a hyper-depolarization index (HDi, see methods 3.6.1). Values close to 1 stand for a strong imbalance between the hyper- and depolarization, while values close to 0 stand for almost equal hyper- and depolarization (see the corresponding values for the example cells in Fig.5.1A-C). The population distribution of the hyper-depolarization index is shown in Figure 5.1D and ranged between 0-1 $(\mathrm{n}=23)$. Almost $70 \%$ of our cells showed values higher than 0.4 , meaning that they showed much smaller amount of hyperpolarization to the non-preferred stimulus compared to the depolarization to the preferred contrast. Note, for almost all cells the observed imbalance came from a higher depolarization (depolarization $>$ hyperpolarization). For two cells, the imbalance came from a higher hyperpolarization (hyperpolarization $>$ 
depolarization). One of the two cells was the ON cell described in in Figure 4.2, here the hyperpolarization to the non-preferred stimulus was much stronger than the depolarization to the preferred stimulus.

A
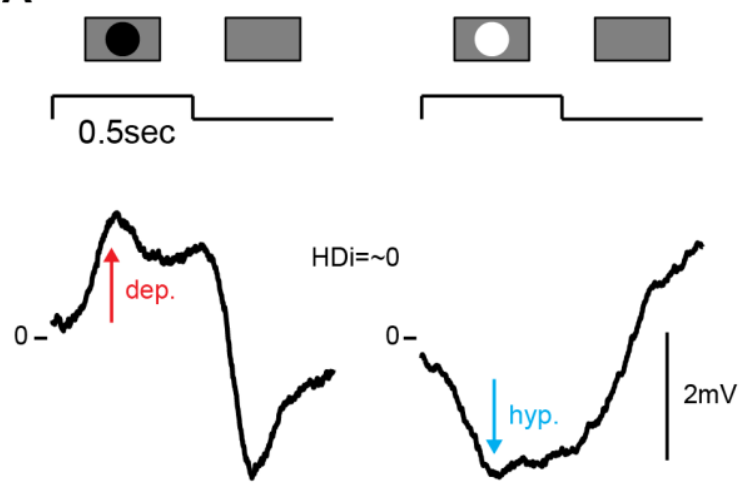

C

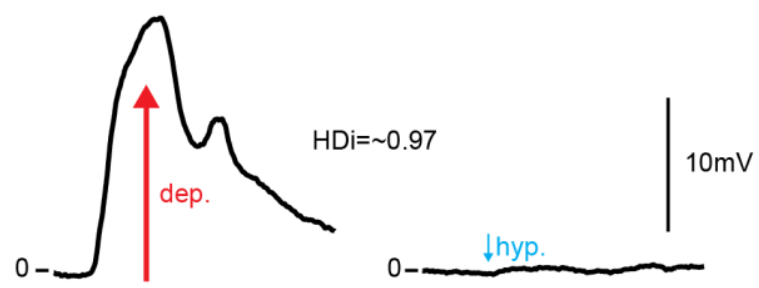

B
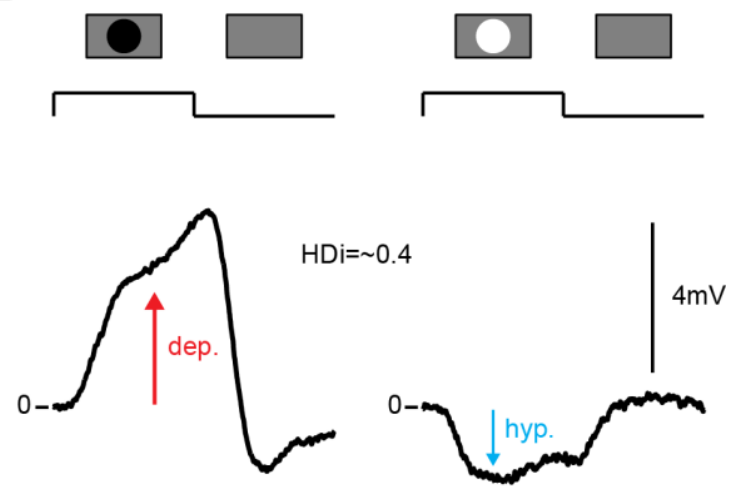

D

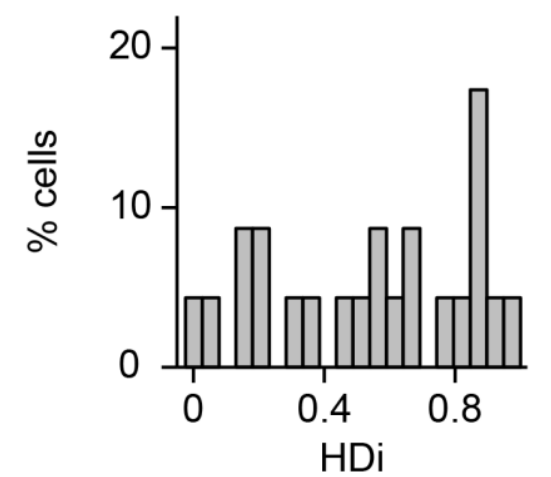

Figure 5.1. Output nonlinearity measured with spots. Comparison of the amount of depolarization (dep., red) and hyperpolarization (hyp., blue) to a black and white spot of $1 \mathrm{~mm}$ diameter. A. Example cell where the amount of depolarization and hyperpolarization to the black and white spot is very similar. B. Example cell that hyperpolarize, yet, the amount of depolarization is larger. C. Example cell with strong depolarization and zero hyperpolarization. D. Population distribution of the hyper-depolarization index (HDi, range 0-1, $\mathrm{n}=23$ ), small values stand for an equal amount of hyper- and depolarization (like example cell in $\mathrm{A}$ ), large values stand for an unequal amount (example cells B and C).

In Figure 5.1, we studied the imbalance between the hyper- and depolarization with the largest spot stimulus of $1 \mathrm{~mm}$. Thus, we activated the center and surround together. We have seen in subchapter 4.4 that the activation of the surround can change the temporal properties. Thus, we were wondering, whether the observed imbalance is influenced by the stimulation of the surround and investigated the imbalance during a stimulation limited to the center.

Figure 5.2A shows for an example cell the response traces to the large and small spot for both contrasts. For both spot sizes, the cell showed a similar ratio between the hyper- and depolarization. Over all recorded bipolar cells, we observed a clear correlation between the 
hyper-depolarization index (HDi) during center and large spot stimulation ( $\mathrm{r}=0.87, p<0.001$, $\mathrm{n}=17$, Fig.5.2B). Further, we compared the imbalance in the hyper- and depolarization observed under a spot stimulus, with the imbalance during the full-field light steps. The ratio between the hyper- and depolarization stayed similar during a center and full-field stimulation $(p=0.33, \mathrm{n}=17$, Fig.5.2C). Therefore, we concluded that the degree of imbalance between hyper- and depolarization, is similar under center and full-field stimulation and not changed by the surround.

A

large spot
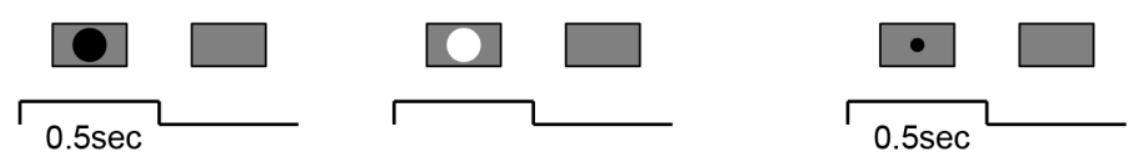

small spot
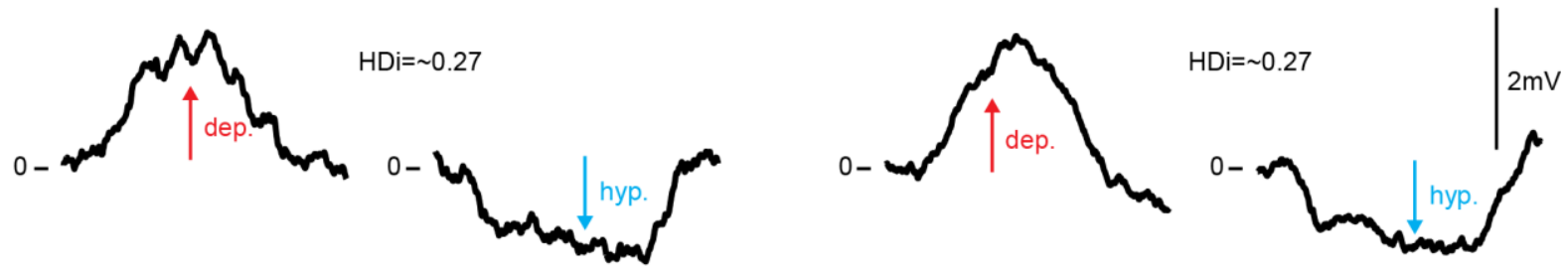

B

hyper.-depolarization index

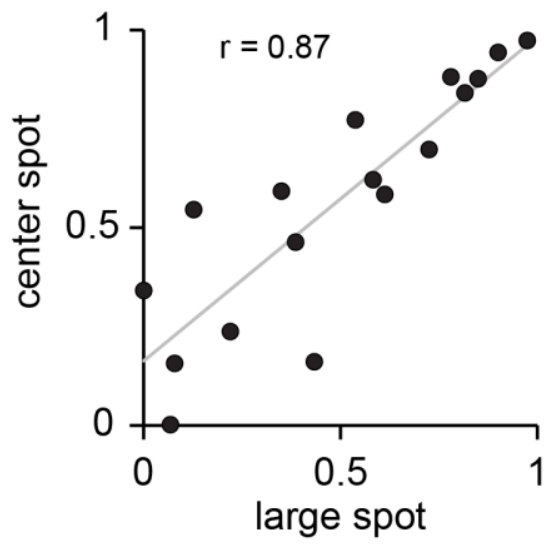

C

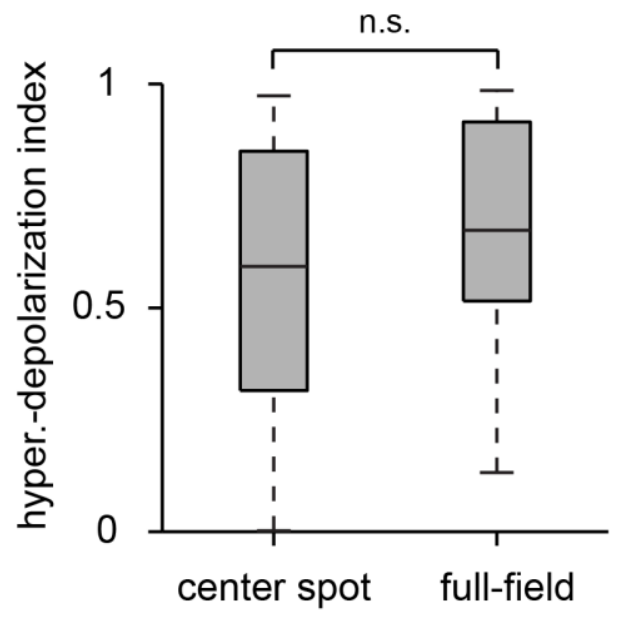

Figure 5.2. Output nonlinearity under center and surround stimulation. A. Example cell where the amount of depolarization and hyperpolarization was similar under a large spot $(1000 \mu \mathrm{m})$ and center spot $(160 \mu \mathrm{m})$. B. Population comparison of the hyper-depolarization index (HDi) under center and large spot stimulation, a clear correlation was observed $(\mathrm{r}=0.87, p<0.001, \mathrm{n}=17)$. C. Population comparison of the HDi under a center spot and full-field light steps, the indices were similar, no significant difference was observed $(p=$ $0.33, \mathrm{n}=17)$. 


\section{Excurse: Gray or no gray?}

As for the receptive field center-surround characterization, 9 out of the 23 cells in Figure 5.1D were recorded with spots that switched between black and white. For 7 cells we recorded both stimuli and again tested, whether the different stimulus designs had an effect on the imbalance between the hyper- and depolarization. We found that the hyper-depolarization index (HDi) was strongly correlated between the two stimulus designs (Fig.5.3A, r= 0.83, $p=0.02, \mathrm{n}=7$ ). As for the receptive field center-surround characterization, we excluded the possibility that the intermediate gray screen can change the imbalance between the hyper-depolarization. Note however, we found some cells, like the example in Figure 5.3B-C, where the hyper-depolarization ratio was more balanced during the black-white switch (Fig.5.3C, $+4 \mathrm{mV}$ depolarization, $-4 \mathrm{mV}$ hyperpolarization) than during the stimulus with gray intermediate screen (Fig. 5.3B, $+5 \mathrm{mV}$ depolarization, $-2 \mathrm{mV}$ hyperpolarization).

A

hyper.-depolarization index

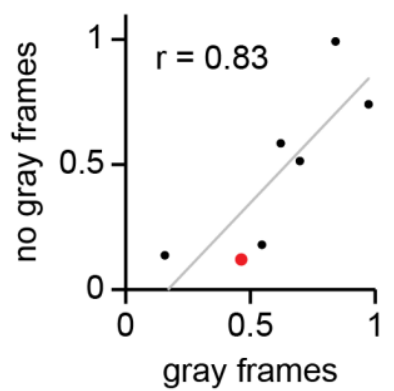

B

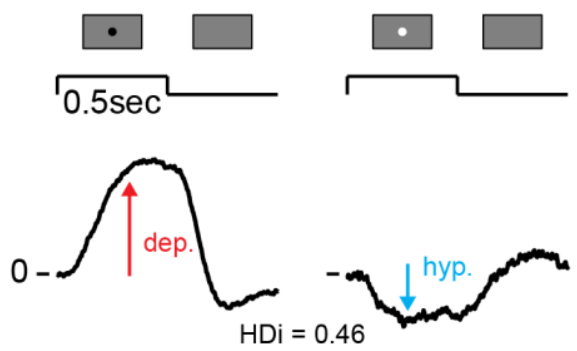

C

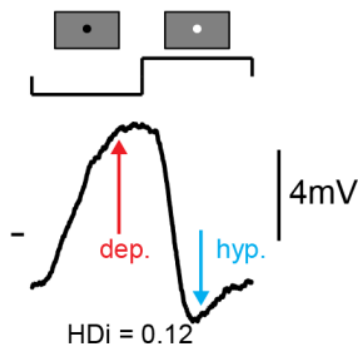

Figure 5.3. Output nonlinearity with different spot designs. A. Positive correlation among the hyper- depolarization indices (HDi) for spots with gray frames and spots without gray frames $(r=0.83, p=0.02, n=7)$. B. Example cell under a center spot with gray preframes, the cell showed an unequal amount of hyper- and depolarization. C. Same cell as in B but under a center spot with a black-white switch (no gray-frames), here the cell showed a more equal amount of hyper- and depolarization.

\subsubsection{Nonlinearities measured with white noise}

Another way to assess the imbalance between the hyper- and depolarization is with white noise analysis. With the spots, we only studied the nonlinear transformation for the highest contrast changes $( \pm 100 \%)$ but not for intermediate contrast values. The white noise analysis provides an approach to study those intermediate contrast values too. From the computed spatial and temporal filters of the white noise analysis one can deduce the output response function (see methods 3.6.1). The computed output function tells us how the membrane potential of a cell changes for a particular incoming stimulus sequence. For example, the cell in Figure 5.4A 
increases the membrane potential by $1 \mathrm{mV}$ (depolarization compared to the baseline level) when the incoming stimulus sequence matches the cell's filter. If, on the other hand, the stimulus sequence is opposite to the filter, the cell decreases the membrane potential by $1 \mathrm{mV}$ (hyperpolarization compared to the baseline level). In this example, we have a linear output transformation (seen in the linear shape of the function).

We also observed bipolar cells with nonlinear output functions. For the example cell in Figure 5.4B, a stimulus sequence that matches the cell's filter depolarized the membrane potential by $\sim 3 \mathrm{mV}$. The stimulus sequence opposite to the filter hyperpolarized the membrane potential by $\sim 1 \mathrm{mV}$. We quantified the degree of nonlinearity of the output function with an output nonlinearity index $(\mathrm{ONi})$. The index takes values close to 0 for linear transformations and values close to 1 for nonlinear transformations (see the corresponding values for the example cells in Figure 5.4 and methods 3.6.1). Figure 5.4C shows the distribution of the absolute value of the nonlinearity index over all cells, ranging from indices of $\sim 0$ to indices of $\sim 0.9$ ( $\mathrm{n}=31$ ).

To study the influence of the surround under white noise stimulation, we tested whether the degree of nonlinearity of the output function changes between a spatio-temporal white noise and a full-field white noise stimulation (Fig.5.4D-F). We found that the directional nonlinearity index (see methods 3.6.1) observed during binary white noise matches the directional nonlinearity index observed during full-field white noise (Fig.5.4F). These results support the findings observed with spots. The degree of nonlinearity seems to be similar under center and full-field stimulation and not significantly influenced by the surround. 
A

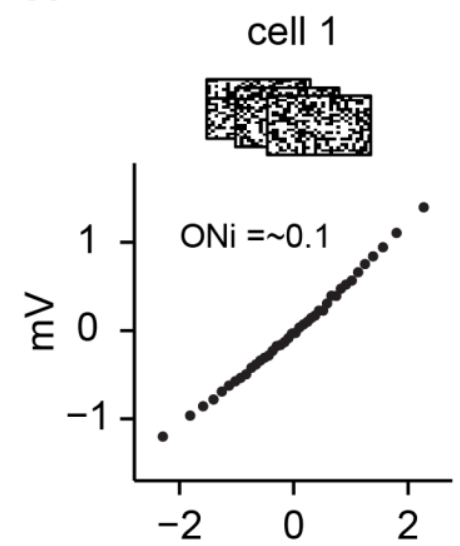

D
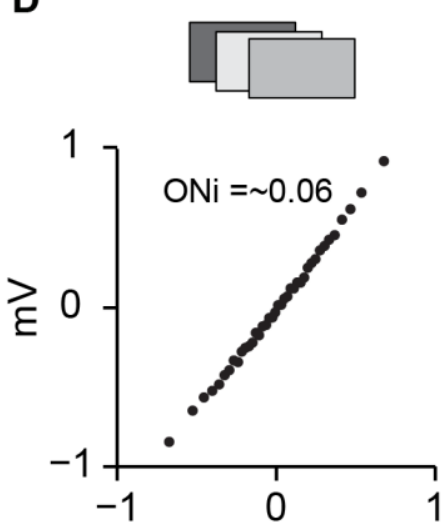

filtered stimulus
B

cell 2

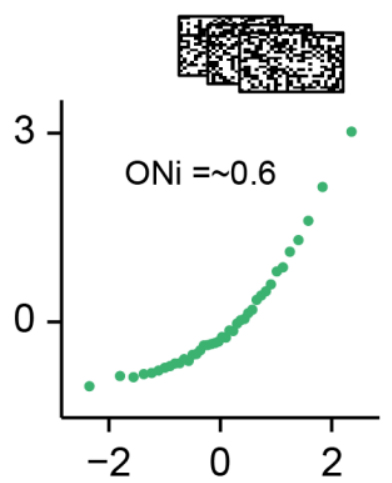

E
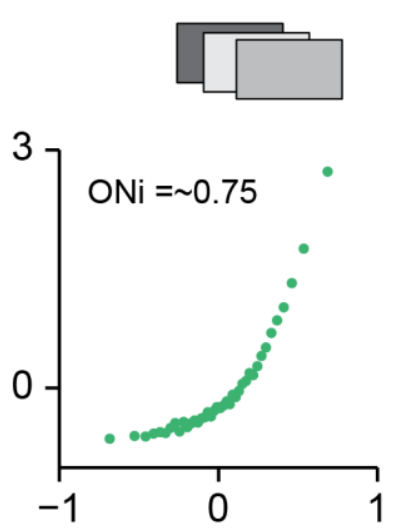

filtered stimulus
C population data

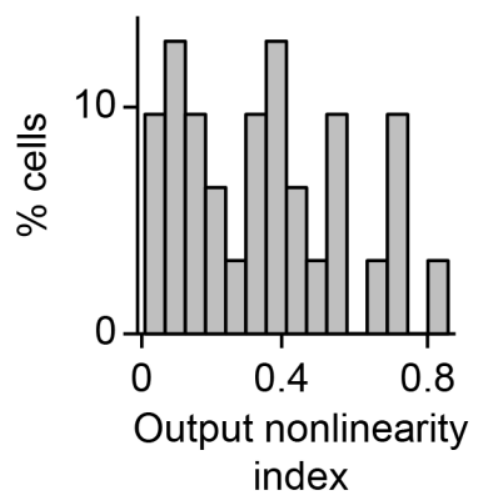

$\mathbf{F}$

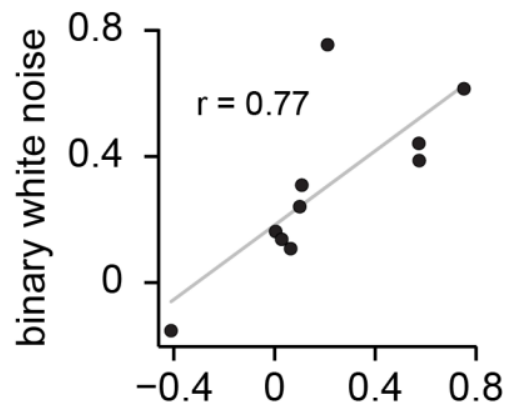

full-field white noise

Figure 5.4. Output nonlinearity with white noise. Stimulus-response transformation from binary (A-C) and fullfield (D-E) white noise. A. Example cell with a linear stimulus-response transformation under binary white noise $(\mathrm{ONi}=\sim 0.1)$. B. Example cell with a nonlinear stimulus-response transformation $(\mathrm{ONi}=\sim 0.6)$. C. Population distribution of the output nonlinearity index $(\mathrm{ONi})$ under the binary white noise stimulus (range $0-0.9, \mathrm{n}=31)$. D. Same linear cell as in A but under a full-field white noise stimulation. E. Same nonlinear cell as in B under a full-field white noise stimulation. F. Positive correlation of the directional ONi under a full-field and binary white noise stimulation $(\mathrm{r}=0.77, p=0.01, \mathrm{n}=10)$.

\subsubsection{Are the nonlinearities with spots connected to the nonlinearities with white noise?}

Previous studies using white noise reported linear bipolar cell responses, while studies using spots reported nonlinear responses (e.g. Rieke (2001) vs. Fahey and Burkhardt (2003)). Therefore, we tested whether the observed nonlinearities with spots are connected to the observed nonlinearities with white noise. We indeed found a positive correlation between the nonlinearities values measured with the two different light stimuli (Fig.5.5C r $=0.72, p=0.001$, $\mathrm{n}=16$ ). As illustrated with an example in Figure 5.5A, bipolar cells showing a balanced hyper- and 
depolarization with spots also showed a linear output response transformation with binary white noise. Further, bipolar cells showing an imbalance in hyper- and depolarization showed a nonlinear stimulus-to-response function (Fig.5.5B). The clear correlation shows that the nonlinearities can be observed with both types of stimuli. Therefore, for further analysis, we only use the white noise responses to quantify the bipolar cells' output nonlinearity.

A

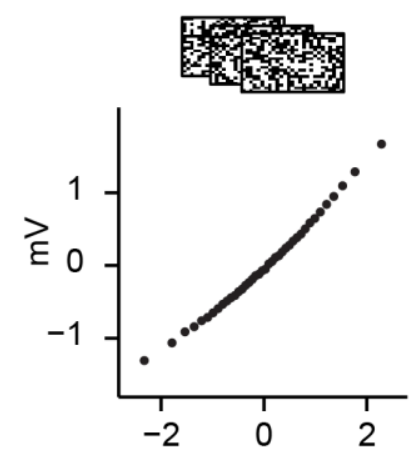

B

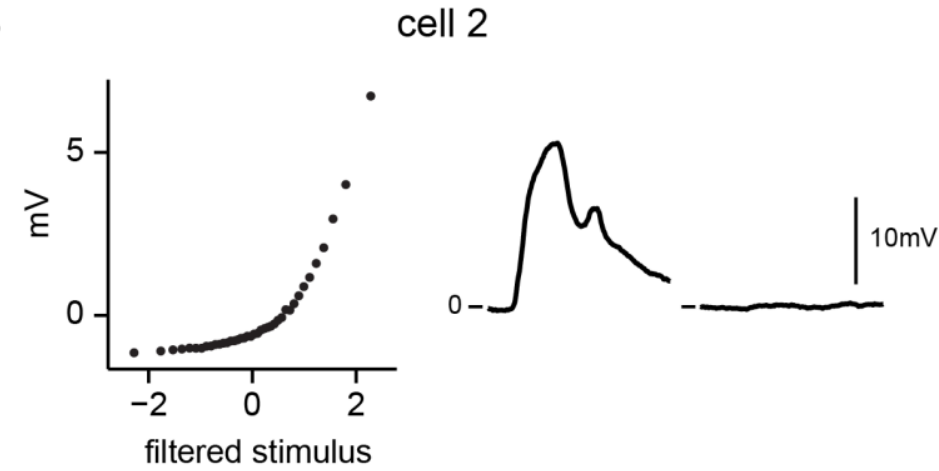

cell 1
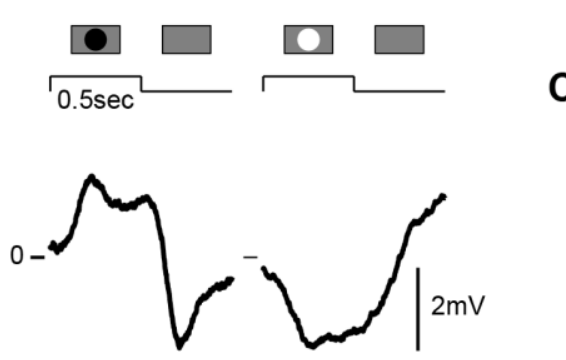

C nonlinearity index

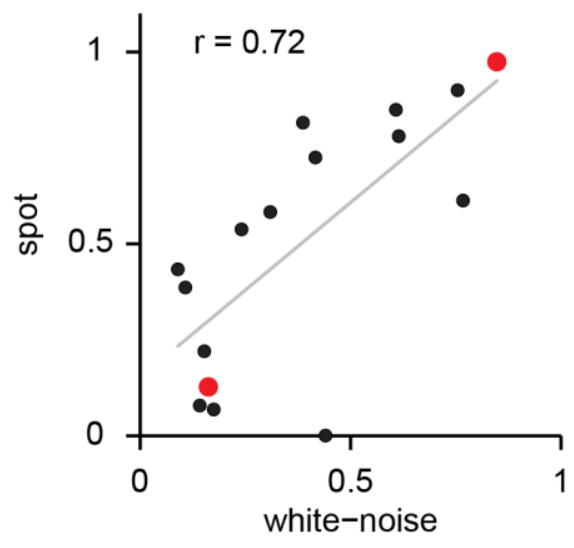

Figure 5.5. Comparison of the nonlinear output transformation with spots and white noise. A. Example cell showing a linear stimulus-response transformation with binary white noise and spots. B. Example cell showing a nonlinear transformation with binary white noise and spots. C. Positive correlation between the absolute nonlinearity indices under white noise and spot stimuli $(\mathrm{r}=0.72, p=0.001, \mathrm{n}=16)$. Red dots are the two example cells.

\subsubsection{Are the output nonlinearities related to the general response properties?}

It has been speculated that different types of bipolar cells might show different nonlinear output functions (Schwartz and Rieke, 2011). Yet, this question has never been studied. Thus, we investigated whether any of the previously described general response characteristics (chapter 4) are connected to the nonlinearities observed in the output function. 
Interestingly, we found that the more sustained the bipolar cells were during a center stimulation, the more linear they were in their output response functions $(\mathrm{r}=-0.88, p=0.002, \mathrm{n}=9$, see Fig.5.6A). Further, we found, that the faster the bipolar cells responded to the center spot, the more linear were their response functions $(r=0.89, p=0.001, n=9$, see Fig.5.6C). Thus, these results indicate that the degree of nonlinearity in the output function might be cell type specific.

We have documented that the surround can change the temporal properties of a cell (subchapter 4.4). Thus, we were curious whether the reported relationships were still present under a surround activation. We observed that the relationship of the output nonlinearity index with the latency completely disappeared when comparing it to the large spot $(\mathrm{r}=0.25, p=0.5, \mathrm{n}=9)$ or full-field light steps $(\mathrm{r}=0.21, p=0.3, \mathrm{n}=26)$ (see Fig.5.6D). Further, when comparing the sustained-transient index under a surround stimulation to the output nonlinearity index, the relationship was present, but less pronounced. For example for the large spot we observed a trend $(\mathrm{r}=-0.59, p=0.09, \mathrm{n}=9)$ and for the full-field light steps we observed a smaller correlation $(\mathrm{r}=-0.48, p=0.01, \mathrm{n}=26)$ (see Fig.5.6 B). These results indicate that the temporal response characteristics of the receptive field center are crucial to understand the origin of the nonlinear stimulus-response transformation. The surround seems to play a minor role in shaping such nonlinear input-output functions. For the other response characteristics e.g. diameter size, surround strength, response polarity, we did not find a connection. 
A

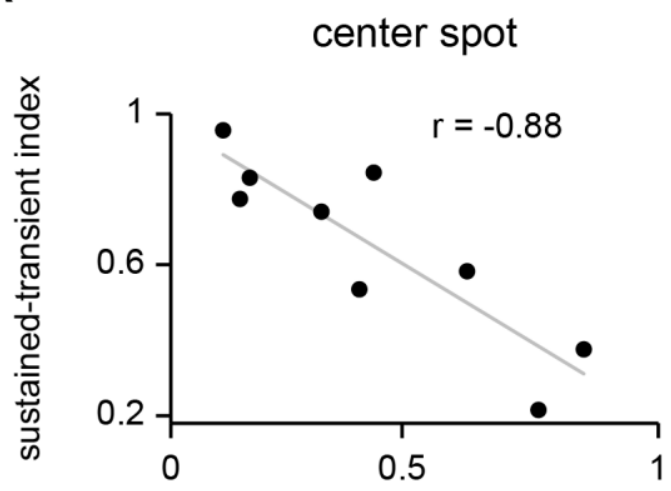

C

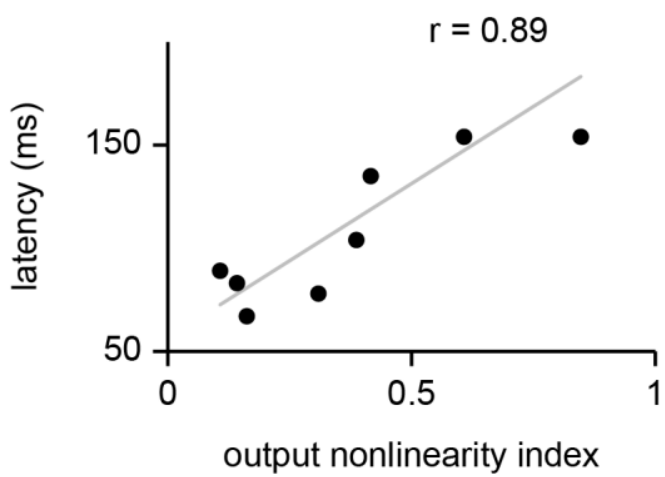

B

large spot

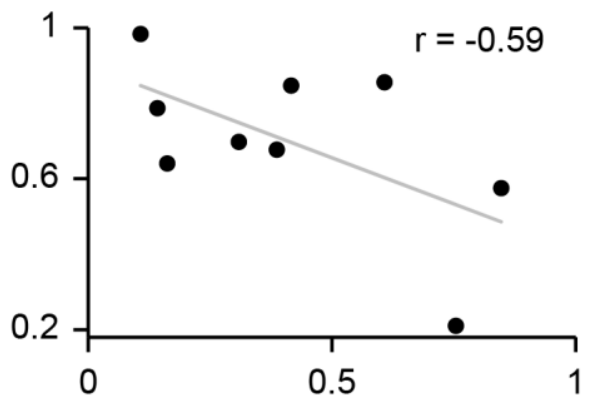

D

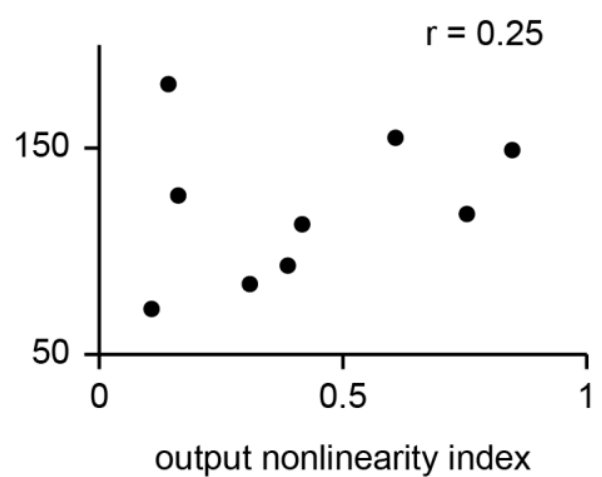

Figure 5.6. Output nonlinearity and the relation to general response properties. A. Negative correlation of the output nonlinearity with the sustained-transient index under a center spot stimulation $(r=-0.88, p=0.002, n=9)$. B. Under the large spot the clear relationship with the sustained-transient index was only present as a trend $(r=-0.59, p=0.09, n=9)$. C. Positive correlation between the response latency and the output nonlinearity under a center spot stimulation $(\mathrm{r}=0.89, \mathrm{p}=0.001, \mathrm{n}=9)$. $\mathrm{D}$. The relationship with the latency was not visible under a large spot stimulation $(\mathrm{r}=0.25, \mathrm{p}=0.5, \mathrm{n}=9)$.

\subsection{Nonlinearities in the spatial integration}

As we have seen, nonlinear signal transformation can occur at two stages in bipolar cells: the output and the inputs. In the previous subchapter, we investigated the output nonlinearities of bipolar cells. In this subchapter, we study the nonlinearities at the inputs to bipolar cells by measuring the spatial integration properties of bipolar cells. To do so, we performed similar experiments that have originally been done in ganglion cells (Enroth-Cugell and Robson, 1966), in bipolar cells. How bipolar cells integrate information over different spatial locations has not yet been investigated. Therefore, we first studied the spatial integration properties under a center stimulation (section 5.2.1) and then under a surround stimulation (section 5.2.2). Further, we 
investigated how the output and input nonlinearities are connected to each other (section 5.2.3) and whether bipolar cells with different general response characteristics (e.g. sustained vs. transient) showed different spatial integration properties (section 5.2.4).

\subsubsection{Spatial integration in the receptive field center}

To investigate whether bipolar cells integrate information in their receptive field center in a linear or non-linear way, we subdivided the spot stimulus that matches the receptive field center size into two halves and stimulated the cells with opposite contrasts in these two subfields (see grating stimulus in Fig.5.7). We periodically reversed the polarity of the two halves at a $1 \mathrm{~Hz}$ rhythm e.g. every half a second one half was switched from black to white, while the other half switched from white to black. If bipolar cells integrate inputs linearly, the stimulus would not excite the cell $(-1+1=0)$. If, however, they integrate inputs nonlinearly, both reversals of the stimulus would excite the cell $(-1+1>0)$.

Figure 5.7B shows the example traces of 4 bipolar cells to the contrast-reversing grating. As can be seen, cell 1 did not respond to the reversing grating, whereas cells 2-4 responded to both reversals of the grating. To compare the magnitude of the response traces, we showed in Figure 5.7A, the responses of the same cells to the flashing spot stimulus that matched the center size. All the four cells clearly responded to the flashing spot; yet they differ in their responses to the reversing grating. 
A
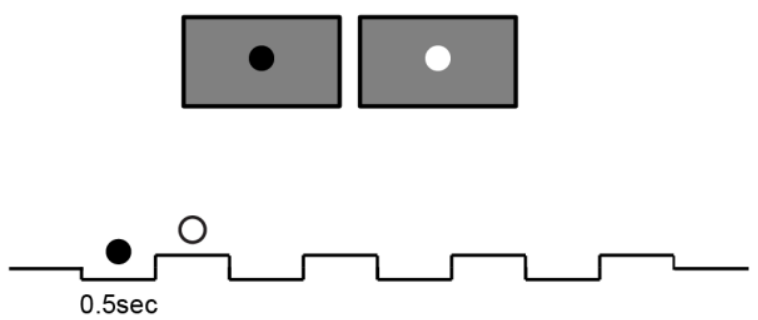

Cell 1
B
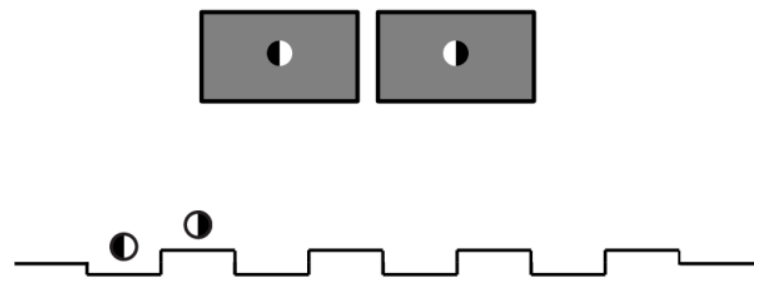
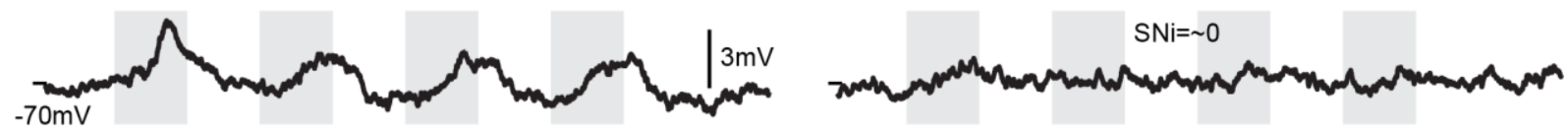

Cell 2

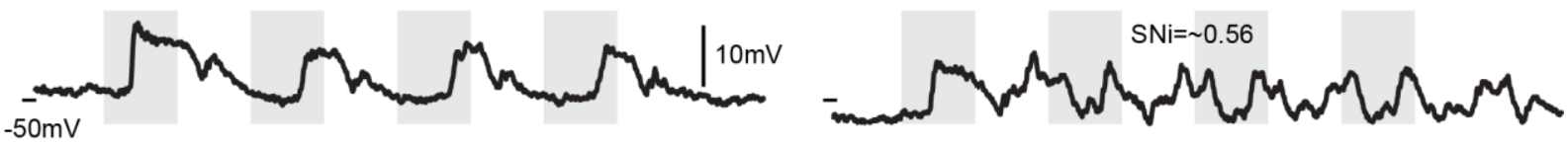

Cell 3
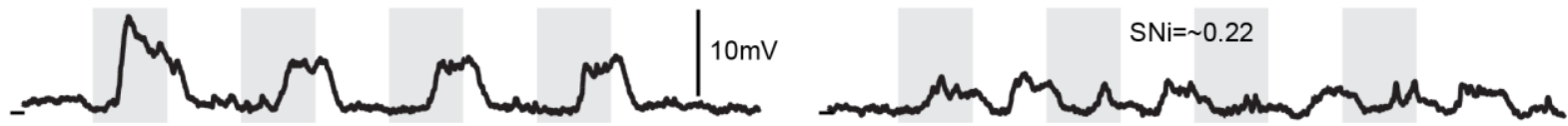

$-40 m \mathrm{~m}$

Cell 4

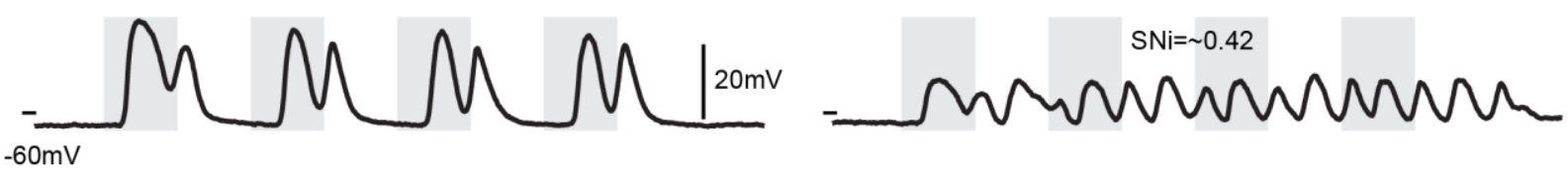

Figure 5.7. Response traces for a linear and nonlinear spatial integration. A. Response traces of four example bipolar cells under a spot stimulus that covered the receptive field center of the cell and switched between black and white every 0.5 seconds. All four cells responded to the stimulus. B. Response traces of the same cells as in A under a spot with half black and half white inside the receptive field center where every $0.5 \mathrm{sec}$ the contrast is reversed. Here, cell 1 does not respond to the stimulus (linear cell), while cells 2-4 responded (spatially nonlinear cells). For each cell, the spatial nonlinearity index (SNi) is shown.

To quantify the responses to the reversing grating, we computed a spatial nonlinearity index by the means of Fourier analysis similar to Hochstein and Shapley (1976b) (see methods section 3.6.2). Cells with a spatial nonlinearity index ( $\mathrm{SNi}$ ) close to 0 did not respond to the reversing grating, while cells with indices larger than zero responded to the grating (see corresponding indices in Fig.5.7B). The population distribution of the spatial nonlinearity index is shown in Figure 5.8 and ranged between 0-0.6 $(n=20)$. Only around 25\% of our cells showed spatial nonlinearity indices of $\sim 0$ and thus did not respond to the grating. The remaining $75 \%$ showed indices larger than zero and responded to the grating with amplitudes of $10-60 \%$ of the spot amplitude, around 25\% showed amplitudes above $40 \%$ of the spot amplitude. 


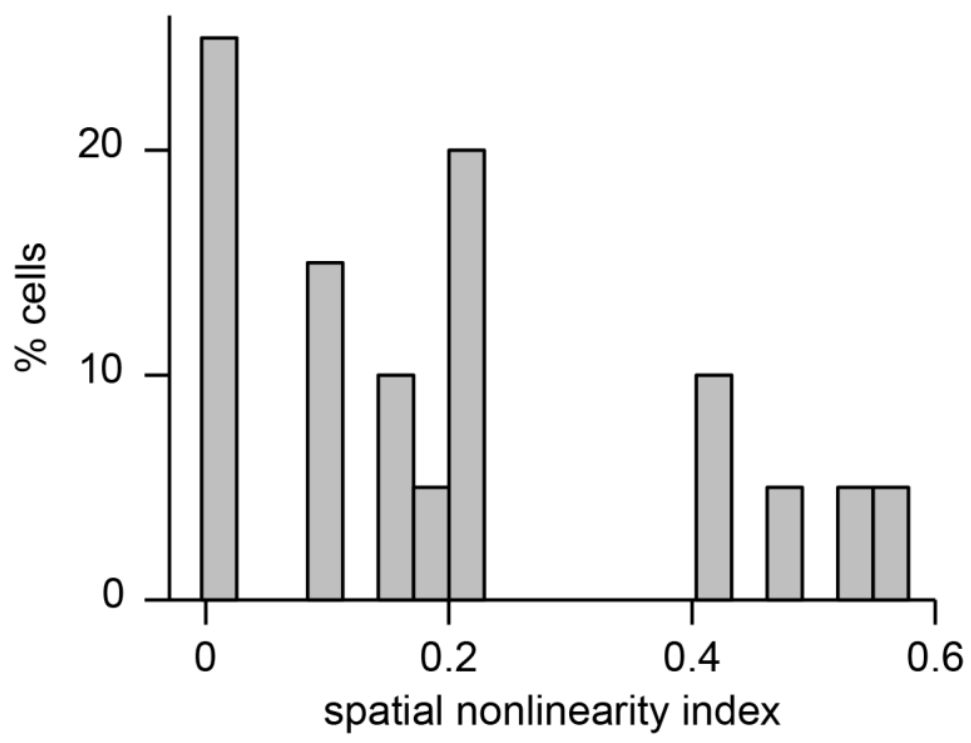

Figure 5.8. Population distribution of the spatial nonlinearity index. Spatial nonlinearity index (SNi), range 0-0.6, $\mathrm{n}=20$.

\section{Scale of spatial integration in the receptive field center}

In a next step, we investigated whether the spatial scale of the reversing grating influenced the integration properties. To do so, we used reversing gratings with fine spatial scales (see Fig.5.9). We separated the receptive field into two halves, into four quarters, into squares of 25 $\mu \mathrm{m}$ and for some cells squares of $10 \mu \mathrm{m}$.

For cells that showed nonlinear spatial integration, we found that the magnitude of the response traces decreased with smaller spatial scales (see example cell in Fig.5.9A). The magnitude is highest with a spatial arrangement of two halves, with an arrangement of four quarters the magnitude decreases but is still present and usually with squares of $25 \mu \mathrm{m}$, the response vanished. For the linear cells, the responses stayed at zero for all different spatial arrangements (Fig.5.9B). In Figure 5.9C, we illustrate the change of the spatial nonlinearity index for different spatial scales for all bipolar cells $(n=20)$. We see that bipolar cells mostly responded to spatial gratings with 2 halves, with 25 or $10 \mu \mathrm{m}$ pixels the responses to the grating become close to zero. Thus, the spatial scale seems to influence the integration properties. When the receptive field of a bipolar cell is separated into two halves, multiple photoreceptors will be exposed only to the black halve and other photoreceptors are exposed only to the white halve. Few photoreceptors will be exposed to half black and half white inside their receptive field. Yet, for a spatial scale of 25 or $10 \mu \mathrm{m}$, which is roughly the receptive field size of a photoreceptor, many photoreceptors will be exposed to half black and half white inside their receptive field. For these small spatial scales the light signals seem to cancel inside the photoreceptor and therefore the bipolar cell does not respond. Thus, the strong decrease in the nonlinearity index for gratings 
with spatial squares of $25 \mu \mathrm{m}$ indicates that photoreceptors integrate light in a linear way over their receptive field.

A
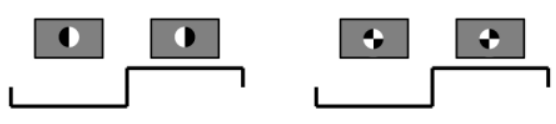

Cell 1

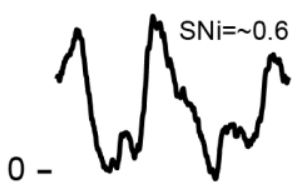

B

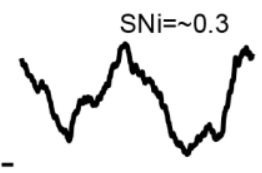

Cell 2

$\mathrm{SNi}=\sim 0$
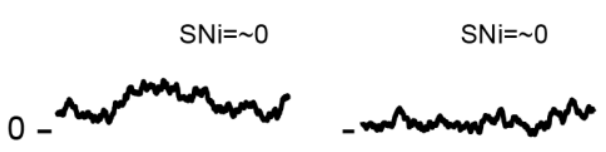
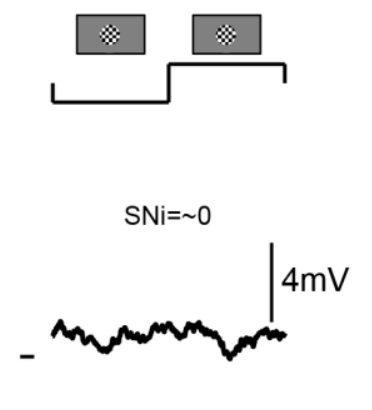

C

\section{Population data}

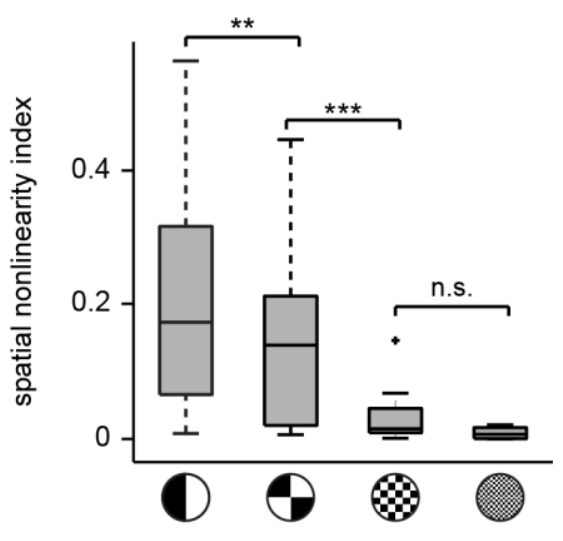

Figure 5.9. Spatial scale of nonlinear integration. A. Response traces of a bipolar cell to patterns with different spatial scales. The cell strongly responded to a spatial scale of two halves inside the receptive field $(\mathrm{SNi}=\sim 0.6)$ and reduced the response to four quarters inside the receptive field $(\mathrm{SNi}=\sim 0.33)$. To a spatial scale of $25 \mu \mathrm{m}$, the cell does not respond $(\mathrm{SNi}=\sim 0)$. B. Response traces of a linear bipolar cell that does not respond to any spatial pattern. C. The population data for the spatial nonlinearity index (SNi) for all cells $(n=20)$ for the four different spatial arrangements: halves, quarters, $25 \mu \mathrm{m}$ and $10 \mu \mathrm{m}$. The index significantly decreased from the halves to quarters to $25 \mu \mathrm{m}\left(* * p<0.01,{ }^{* * *} p<0.001\right)$.

\subsubsection{Spatial integration in the receptive field surround}

Until now, we have looked at the spatial integration inside the receptive field center. To study whether we observe nonlinear spatial integration under a surround stimulation, we recorded from 6 cells the responses to a reversing full-field grating at various positions (Fig.5.10). In Figure 5.10A-B, we see the response of two cells to the full-field grating. One cell is an OFF cell that responded to the grating when the black contrast fully covered the receptive field center and when both contrasts are balanced inside the receptive field. The other cell is an ON cell (B) that responded when the white bar fully covered the receptive field as well as when both bars were shown inside the receptive field. To study whether a surround stimulation changes the degree of spatial nonlinear integration in the receptive field center, we compared the indices of a center spot stimulation to the indices under a full-field stimulation. We found a positive trend between the center and surround stimulation ( $\mathrm{r}=0.71, p=0.11, \mathrm{n}=6$, see Fig.5.10C). Thus, our results indicate, that the degree of nonlinear spatial integration is similar under center and full- 
field stimulation. Further, the spatial integration property seem to be formed mainly by the properties of the center and likely not by the surround. These findings are similar to the output function, where the degree of nonlinearity remained similar under a center and full-field stimulation.

A
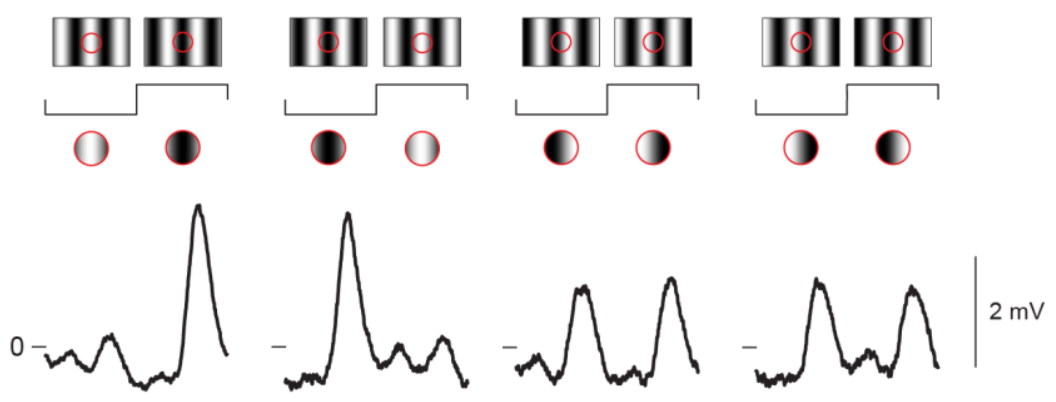

B

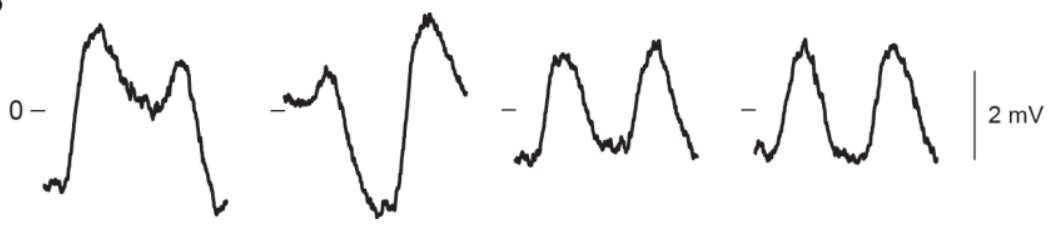

C spatial nonlinearity index

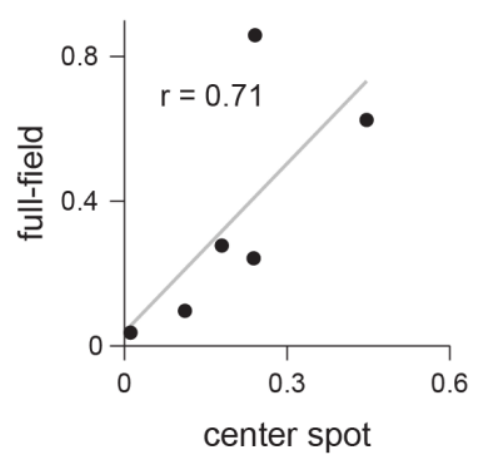

Figure 5.10. Nonlinear spatial integration under surround stimulation. A. Example traces of an OFF bipolar cell under a full-field grating stimulation. The cell responded when the center was covered by a black bar, as well as when half white and half black was shown inside the receptive field. B. Example trace of an ON bipolar cell that responded when the center was covered by a white bar and when half white and half black was shown inside the receptive field. C. Positive trend between the spatial nonlinearity index under center and surround stimulation $(\mathrm{r}=0.71, p=0.11, \mathrm{n}=6)$.

\subsubsection{Is the spatial nonlinearity connected to the output nonlinearity?}

In the previous subchapter 5.1 we have seen bipolar cells with linear and nonlinear output functions. In this chapter, we described bipolar cells with linear and nonlinear spatial integration. Hence, we were curious whether the different types of nonlinearities stand in relationship to each other. In Figure 5.11A, we show an example bipolar cell that integrated information in space linearly and the cell had a linear stimulus-response-function. In Figure 5.11B, we show an example bipolar cell that integrated information in space nonlinearly and here we found a nonlinear stimulus-to-response function. Figure 5.11C shows the correlation between the spatial nonlinearity index and the output nonlinearity index. We found a strong significant correlation between the two types of nonlinearities $(r=0.8, p<0.001, n=19)$. The higher the degree of 
nonlinearity in the output function, the higher the nonlinearity in the spatial integration (the example cells are marked in red).

A

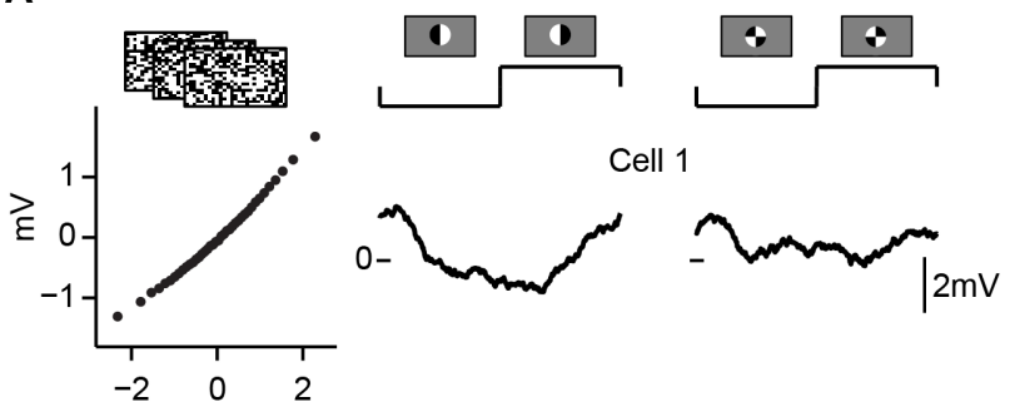

B
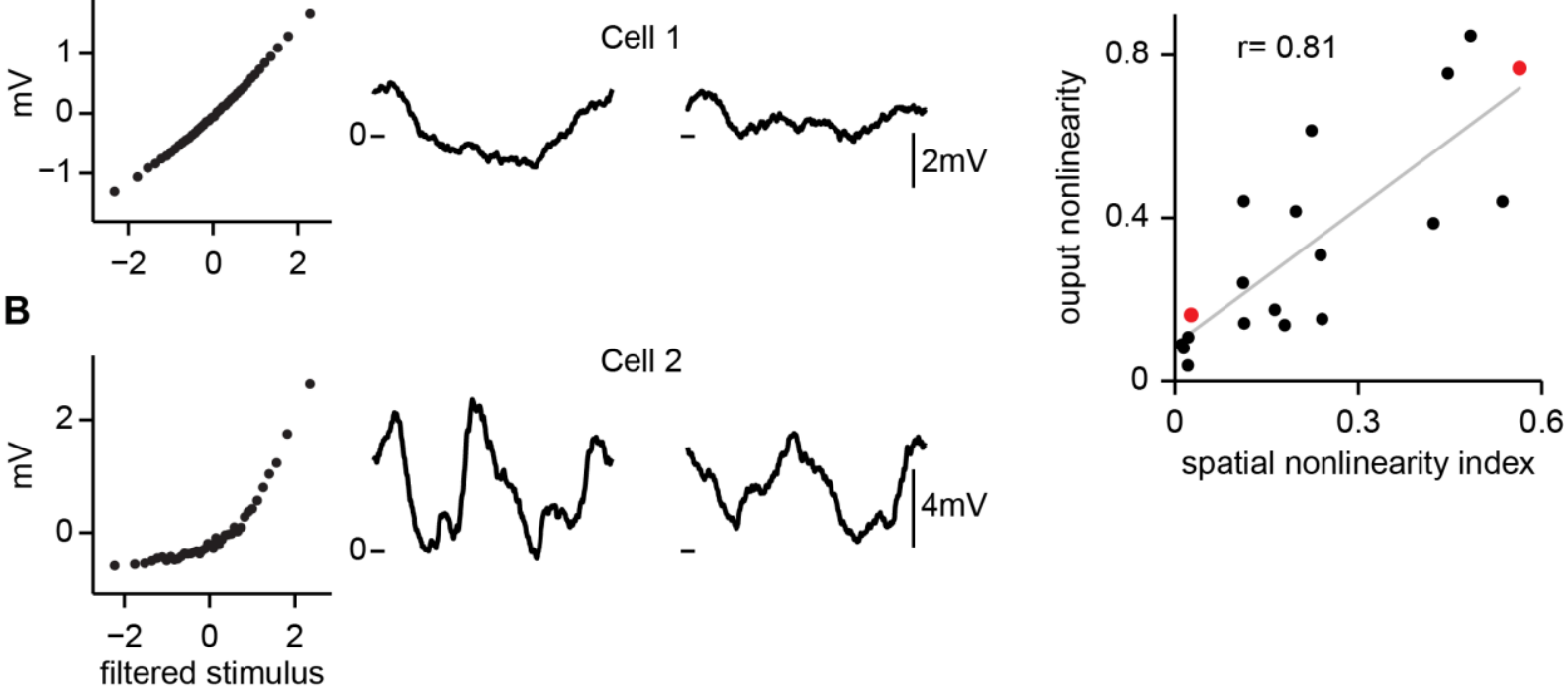

Figure 5.11. Comparison of the spatial nonlinearity and the output nonlinearity. A. Stimulus-response transformation of a linear bipolar cell and the corresponding nulling of the response to the grating stimulus. B. Stimulusresponse transformation of a nonlinear bipolar cell and the corresponding frequency doubling response to the grating stimulus. C. Positive correlation between the spatial and output nonlinearity $(\mathrm{r}=0.8, p<0.001$, $\mathrm{n}=19$, red dots are the example cells in $\mathrm{A}$ and $\mathrm{B}$ ).

These results are curious and point out that the measured output nonlinearity might mainly be driven by the spatial nonlinearity. The results allow for a simple speculation that we briefly want to outline. If the magnitude of hyper- and depolarization in a bipolar cell is similar (e.g. $+2 \mathrm{mV}$ and $-2 \mathrm{mV}$ ), then an integration of a stimulus with half white and half black inside the receptive field would be summed to zero and therefore silence the response. If on the other hand, the magnitude of hyper- and depolarization in a bipolar cell is different (e.g. $+8 \mathrm{mV}$ and $2 \mathrm{mV}$ ), an integration of a stimulus with half white and half black would not be summed to zero (e.g. $+6 \mathrm{mV}$ ) and therefore the cell would respond.

We applied this logic to our recorded bipolar cells and tested whether through a simple summation of the hyper- and depolarization from the flashing spot we can predict the responses to the reversing grating (see the two cells in Fig.5.12). We indeed found that the predicted responses (red dashed line) looked very similar to the real responses of the cells (black line); both for nonlinear and linear cells (Fig.5.12 A-C). We quantified the similarity between the predicted and real response by computing the correlation $(\mathrm{R})$ between the traces and report them as 
explained variance $\mathrm{R}^{2}$ (see coefficients in Fig.5.12 A-C for the example cells and methods). The population distribution of the explained variances is shown in Figure 5.12 D. We found that for around $45 \%$ of our cells we could get explained variances higher than $70 \%$ (red rectangle). Thus, for those cells the predicted traces strongly matches the real traces. These first findings are curious, need however more investigation to for example better understand what happens with the remaining $\sim 50 \%$ of the cells. One of the downside of the quantification with the explained variance is that for linear cells, like the example in Figure 5.12B, even though the predicted and real traces look similar, the coefficient takes very low values $(\sim 0.15)$. One reason for these low values might be due to the internal "noise" of the membrane potential when randomly fluctuating at around 0 , thus not being activated by the stimulus. To predict such a "random" fluctuation that is independent of the stimulus is therefore more difficult. Hence, even if we predict a response with a fluctuation at around 0 , the random fluctuation in the real signal might sometimes be higher and sometimes be lower than the predicted signal and therefore the similarity cannot be measured in a correlation. Further analysis could help to better quantify the prediction performance for these cases of small responses.

A

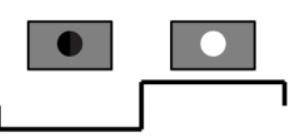

Cell 1
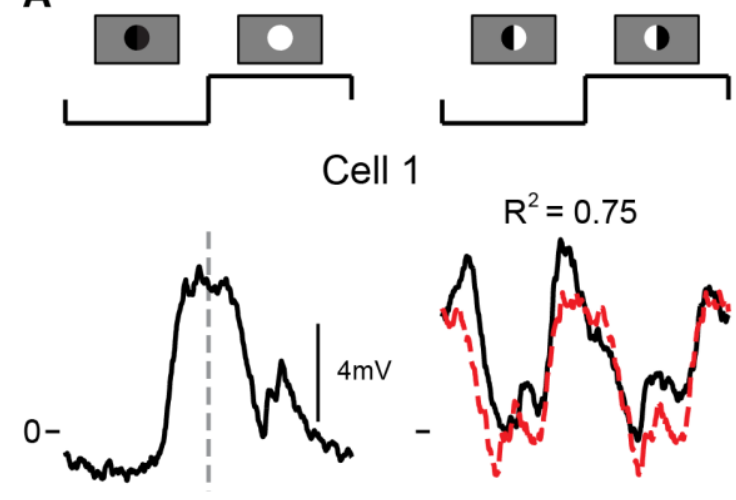

$$
R^{2}=0.75
$$

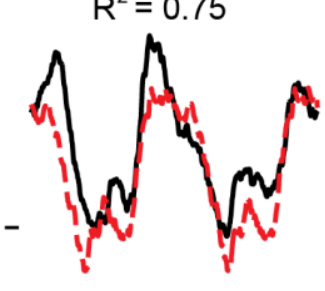

B

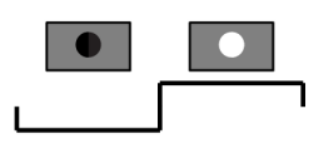

Cell 2
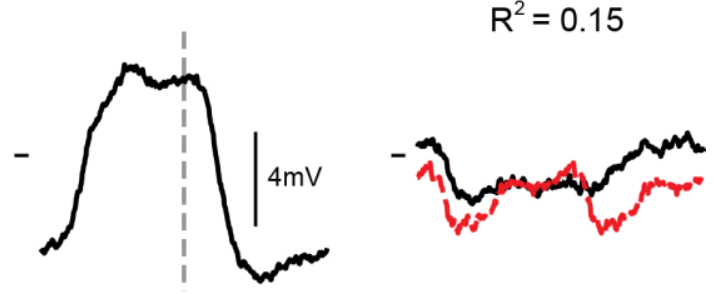

C

Cell 3

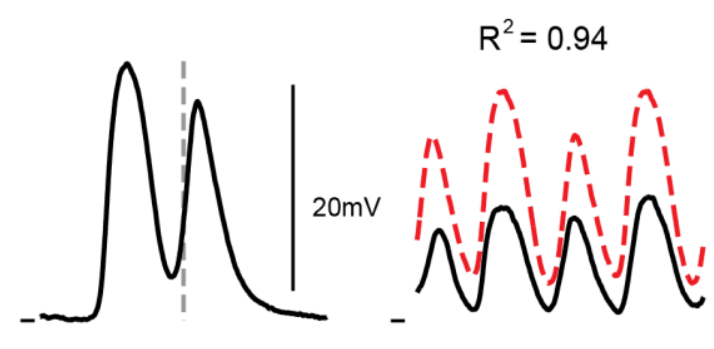

D

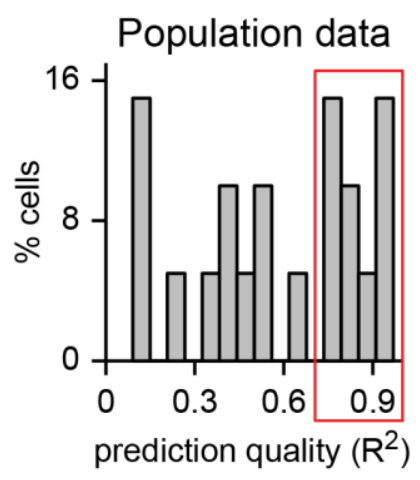

Figure 5.12. Predicting the nonlinear spatial integration with the black- white spot. A.-C. Example cells with the response traces to the spot and the grating as well as the prediction to the grating by summing the response 
to the black and white part of the spot (gray dashed line for the separation). D. Population distribution of the prediction performance $\left(\mathrm{R}^{2}\right)$, range $0.12-0.94, \mathrm{n}=19$.

The prediction was done with the responses to the reversing spot, i.e. the spot switched between black and white thus without gray screen. We also tried to do the same prediction but with the responses to the spot that had a gray screen (Fig.5.13). As expected from the results presented in the subchapter 4.4 , the temporal dynamics of the prediction were different. The cell responded with a faster latency to the spot with gray screen and therefore the predicted peaks are shifted to an earlier time point. The results point out that to predict the responses to a reversing grating, a spot is needed that switches between black and white. However, we speculate that if we would present the grating with an intermediate gray screen (no reversing), we would need a spot that also has a gray screen to correctly predict the temporal dynamics.

A
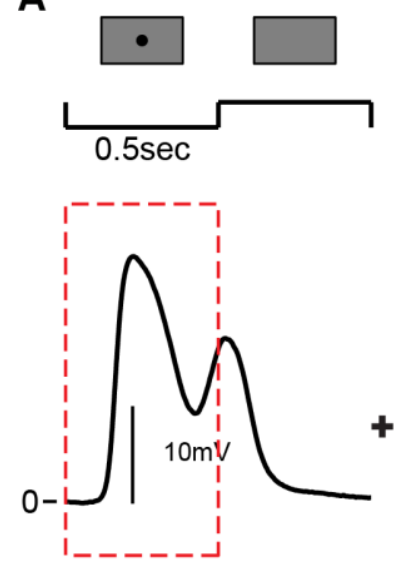
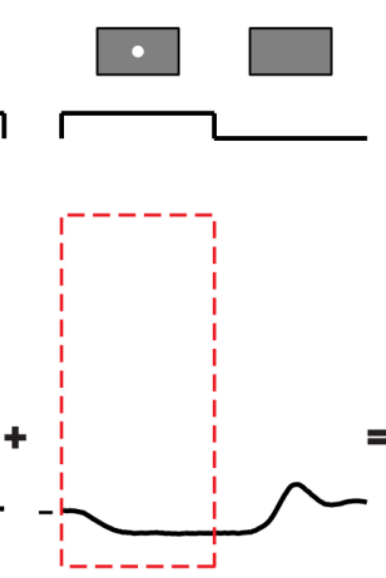
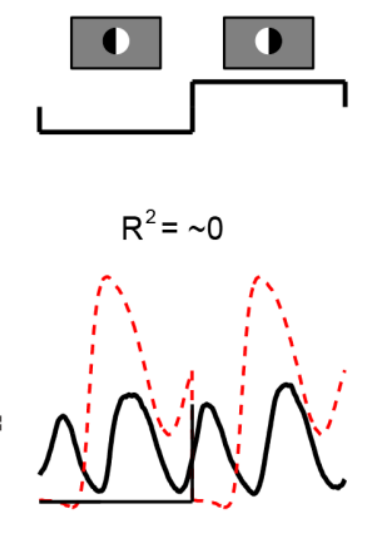

B

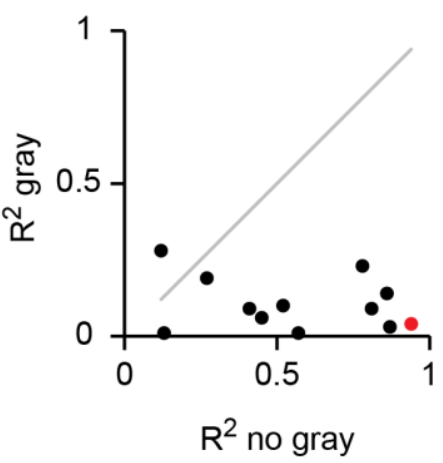

Figure 5.13. Predicting the nonlinear spatial integration with gray preframes. A. Example cell with the response traces to the spot and the grating. Further, the prediction to the grating was done by summing the response to the black and white spot (red dashed line). D. Comparison of the prediction performance with the spot without gray preframes and the spot with gray preframes, gray is the unity line. Clearly, the performance is much better for the spot without gray preframes (red dot is the example cell).

\subsubsection{Is the spatial nonlinearity related to the general response properties?}

We further investigated whether the degree of nonlinear spatial integration is related to the described general response characteristics in chapter 4. Our findings were very similar to the previous analysis of how the output function is related to the general response properties. We found that the more sustained the bipolar cells were during a center stimulation, the more linear 
they were in their spatial integration $(\mathrm{r}=-0.91, p=0.002, \mathrm{n}=8$, see Fig.5.14A). In addition, we found, that the faster the bipolar cells responded to the center spot, the more linearly they integrated spatial information ( $\mathrm{r}=0.73, p=0.04, \mathrm{n}=8$, see Fig.5.14C). Again, these clear relationships were only present under a center stimulation. When comparing the sustainedtransient index of the large spot to the spatial nonlinearity index, the relationship was only present as a trend $(\mathrm{r}=-0.67, p=0.07, \mathrm{n}=8$, see Fig.5.14B), for the full-field light steps, no relationship could be observed $(\mathrm{r}=-0.3, p=0.2, \mathrm{n}=20)$. Further, the relationship of the spatial nonlinearity index with the latency completely disappeared when comparing it to the large spot $(\mathrm{r}=0.07, p=0.86, \mathrm{n}=8$, see Fig.5.14 D) or full-field light steps $(\mathrm{r}=0.08, p=0.73, \mathrm{n}=20)$. For the other response characteristics e.g. diameter size, surround strength, response polarity, we did not find a correlations.

These results again highlight that the response characteristics of the receptive field center are crucial for understanding the origin of spatial nonlinear information integration. The surround seems to play a minor role for a nonlinear integration of the input signals. Further, the results indicate that the spatial integration properties likely are cell types specific.

A

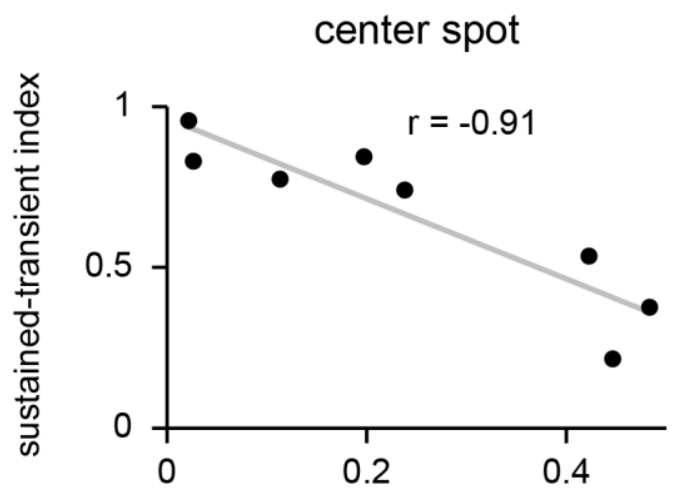

C

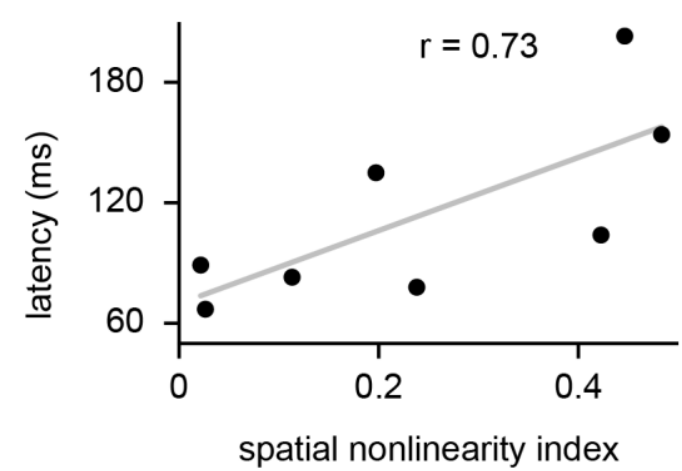

B

large spot

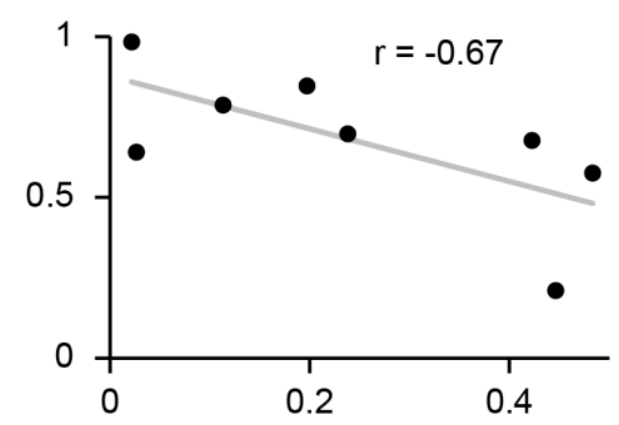

D

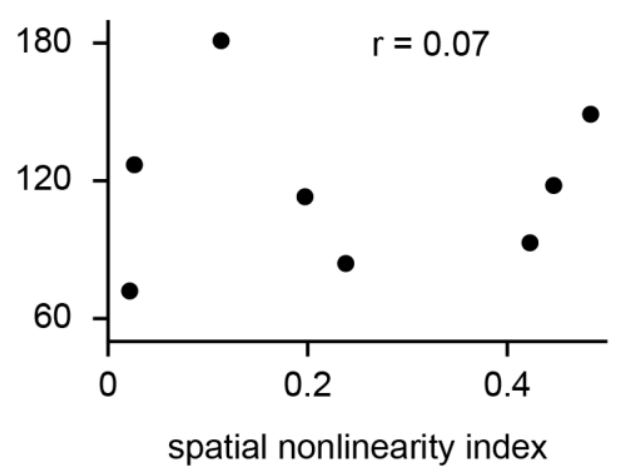

Figure 5.14. Relation of the spatial nonlinearity index to the general response properties. A. Clear negative correlation between the spatial nonlinearity index and the sustained-transient index under a center spot stimulation $(r=-$ 
$0.91, p=0.002, \mathrm{n}=8)$. B. Under the large spot the correlation was only present as a trend $(\mathrm{r}=-0.67, p=0.07$, $\mathrm{n}=8$ ). C. Positive correlation between the response latency and the spatial nonlinearity under a center spot stimulation $(\mathrm{r}=0.73, p=0.04, \mathrm{n}=8)$. D. The relationship was not visible under a large spot light stimulation $(\mathrm{r}=0.07, p=0.86, \mathrm{n}=8)$. 


\section{Linear-nonlinear model in}

\section{bipolar cells}

One of the most important models to understand retinal functions is the linear-nonlinear model (LN-model) (Chichilnisky, 2001; Gollisch, 2013). The model is composed of two stages: 1. linear filter and 2. nonlinear output function. The linear-nonlinear model has mainly been used to study the responses of retinal ganglion cells. Here, three different types of stimuli are investigated: 1. full-field white noise, 2. binary white noise and 3. natural stimuli. It has been reported that the linear-nonlinear model can predict the responses of retinal ganglion cells to fullfield white noise quite accurately (Chichilnisky, 2001; Pillow et al., 2008). However, for stimuli with spatial structure, like the binary white noise or the natural stimuli, the model fails to describe the responses of retinal ganglion cells (Freeman et al., 2015; Liu et al., 2017). One of the reasons for the failure is seen in the nonlinear spatial integration performed by retinal ganglion cells.

Investigating the bipolar cells with the linear-nonlinear model has been largely ignored in the retina field. Bipolar cells are assumed to integrate information linearly in space and therefore it is broadly accepted that their responses should be described by the model accurately. Thus, in our goal 3, we tested whether bipolar cells' responses can indeed be predicted by the linearnonlinear model. To do so, we investigated the linear-nonlinear model with the classical three types of stimuli: full-field white noise (subchapter 6.1), binary white noise (subchapter 6.2) and natural stimuli (subchapter 6.3). 


\subsection{Encoding of uniform light stimuli}

We used a full-field white noise stimulus, which is a spatially uniform contrast sequence with randomized contrast values (see Fig.6.1 and methods). In this subchapter, we show how well the linear-nonlinear model can describe the bipolar cells' responses to such full-field white noise stimuli. First, we describe the prediction performance of the model for two single bipolar cells as well as the population data (section 6.1.1). Then, we investigate the relation of the model performance to the nonlinearities (section 6.1.2) and the general response characteristics (section 6.1.3).

\subsubsection{Prediction performance with uniform light stimuli}

In Figure 6.1A and D, we see the two stages of the linear-nonlinear model (i.e. filter and output function) for two bipolar cells. The filter and output functions are deduced as previously described (section 4.2.2, 5.1.2 and methods). The bipolar cell in Figure 6.1A showed a linear stimulus-response transformation, the bipolar cell in Figure 6.1D exhibited a nonlinear transformation. Further, in Figure 6.1B and E, we see the responses to a new contrast sequence (test set), for the single trials (colored traces) as well as the average (overlaid black trace). Both cells responded very reliably and without strong deviations between trials.

From the built linear-nonlinear model, we then predicted the responses to the new contrast sequence (red dashed traces in Fig.6.1C and F). Clearly, the predicted responses matched the real responses of the cells very closely (black trace). We quantified the similarity by computing the correlation coefficient $(R)$ as explained variance $R^{2}$ between the traces. For both cells the coefficients are very high, for bipolar cell 1 the predicted and real response matched by $97 \%$, for cell 2 by $93 \%$. 

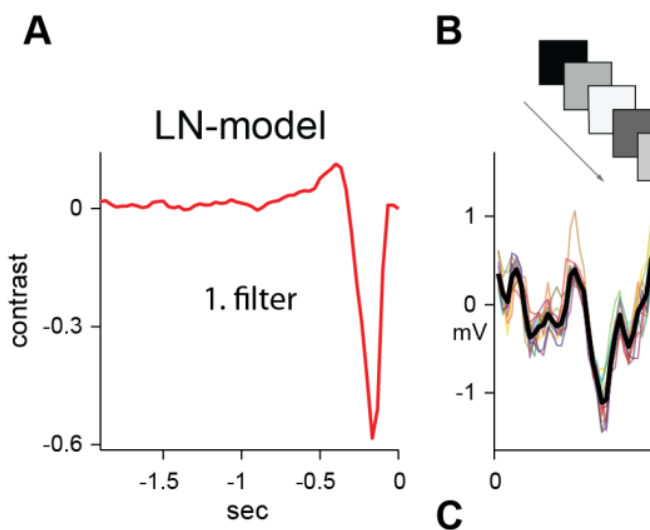

cell 1

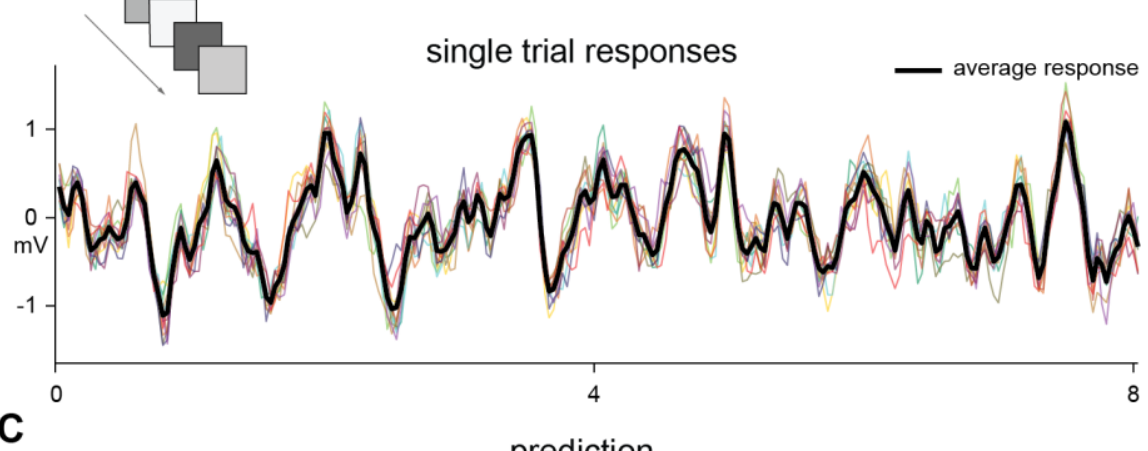

2. output function
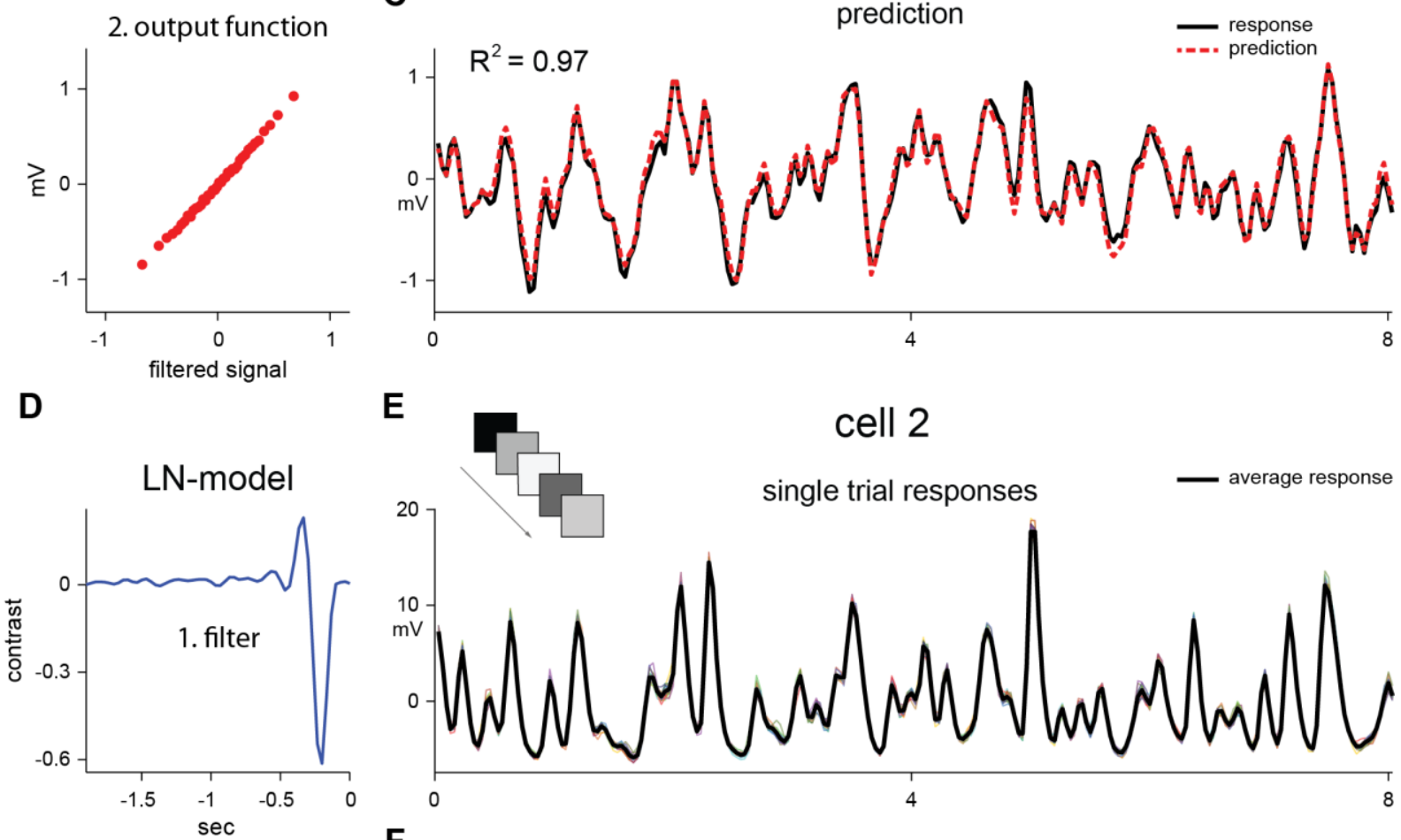

E
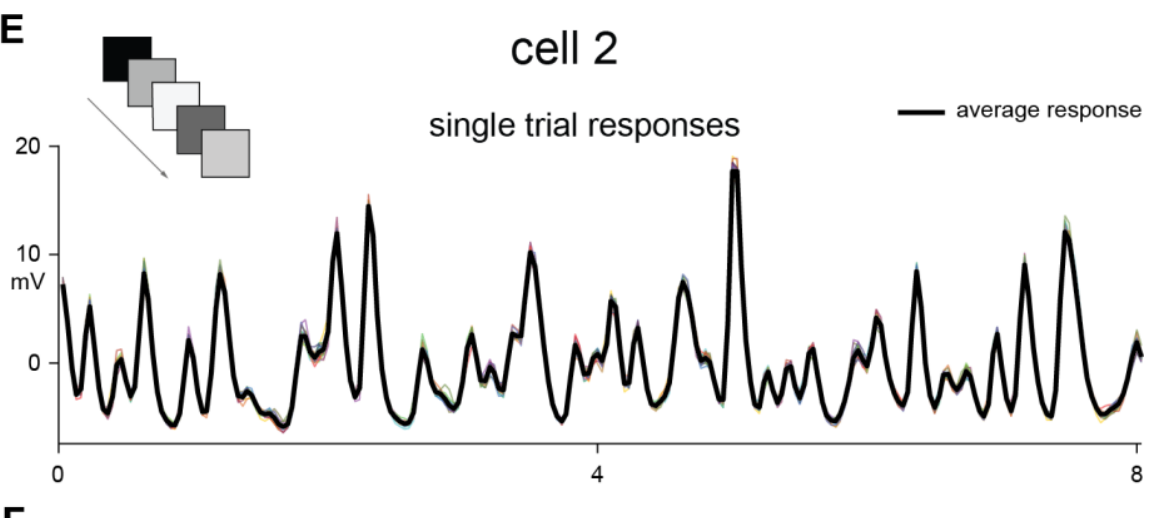

2. output function

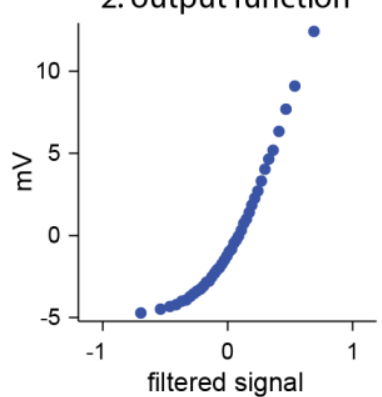

$\mathbf{F}$

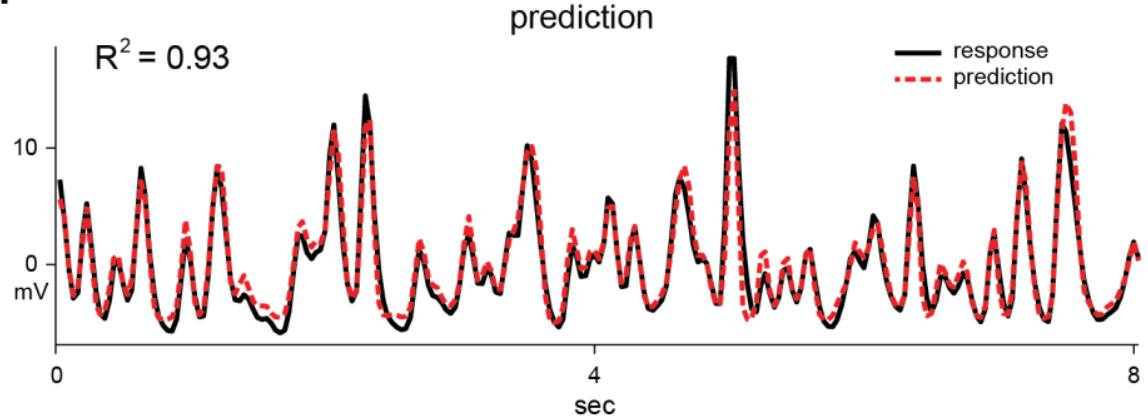

Figure 6.1. Linear-nonlinear model ( $L N$-model) under a full-field white noise stimulation. A. Two stages of the linearnonlinear model (LN-model): filter and output function for example cell 1. B. Single trial responses to the new contrast sequence (colorful traces) as well as the average response (black trace) for the same example cell. C. Predicted response with the LN-model (red dashed line) and the average response (black line) for the cell 1 . The prediction accuracy is $97 \%$. D. LN-model for the example cell 2 with a nonlinear output function. E. Single trial and average responses for the example cell 2. F. Predicted and average response for the example cell match by $93 \%$. 
The population distribution of the prediction performance is shown in Figure 6.2 and varied between $82-98 \%(n=10)$. The mean is around $90 \% \pm 0.05(S D)$, which shows that the responses of bipolar cells to the uniform contrast stimulus can be predicted accurately with the linear-nonlinear model.

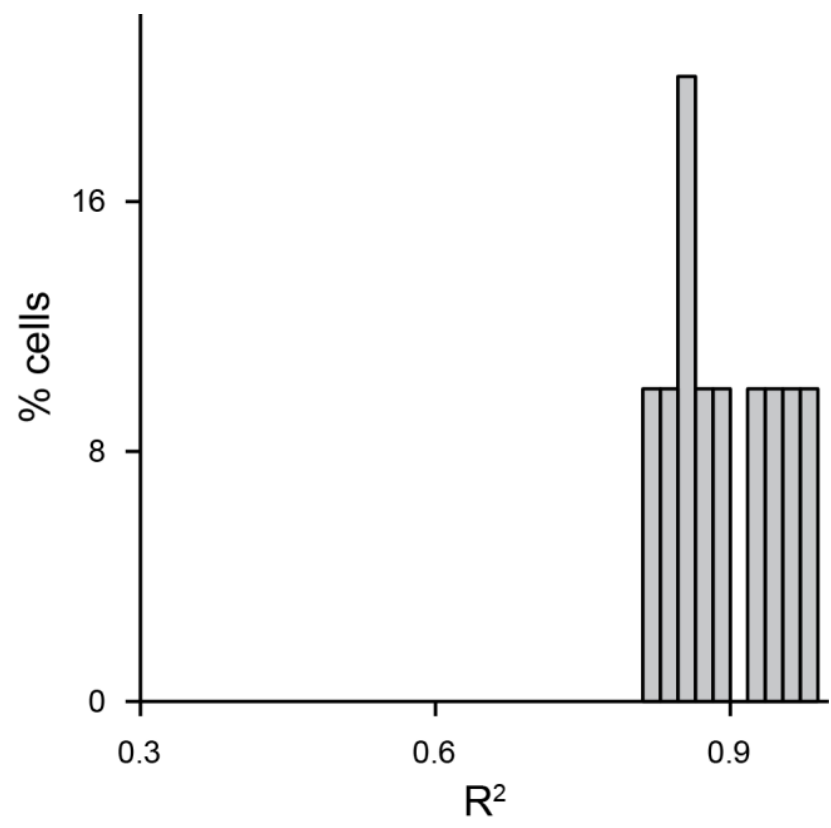

Figure 6.2. Population distribution of the prediction performance with uniform stimuli. Prediction performance is defined as $\mathrm{R}^{2}$. The range is between $82-98 \%$ with 10 bipolar cells.

\subsubsection{Is the prediction performance related to the observed nonlinearities?}

In a next step, we were wondering, whether bipolar cells with a linear output function and a linear spatial integration can be better predicted than nonlinear bipolar cells. Yet, we did not find any correlation between the degree of nonlinearity in the output function and the prediction performance of the linear-nonlinear model. Linear as well as nonlinear cells showed good prediction performances under full-field light stimulation (Fig.6.3A). Further, we also did not find a relation to the degree of nonlinear spatial integration. Cells that integrated information linearly as well as cells that integrated information nonlinearly, showed good prediction performance (Fig.6.3B). We therefore concluded that the degree of nonlinearity in the output function as well as in the spatial integration does not influence the model performance under a full-field light stimulation. 
A

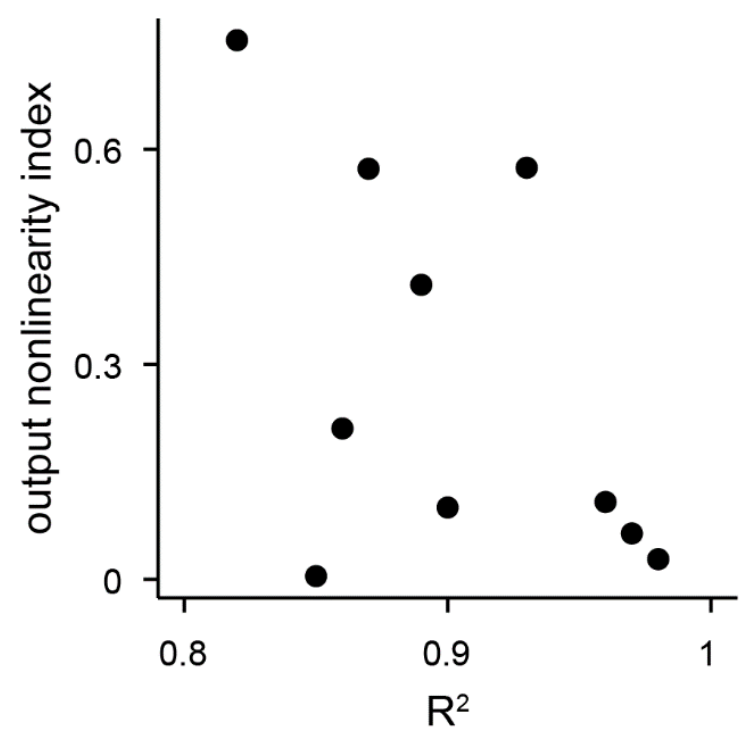

B

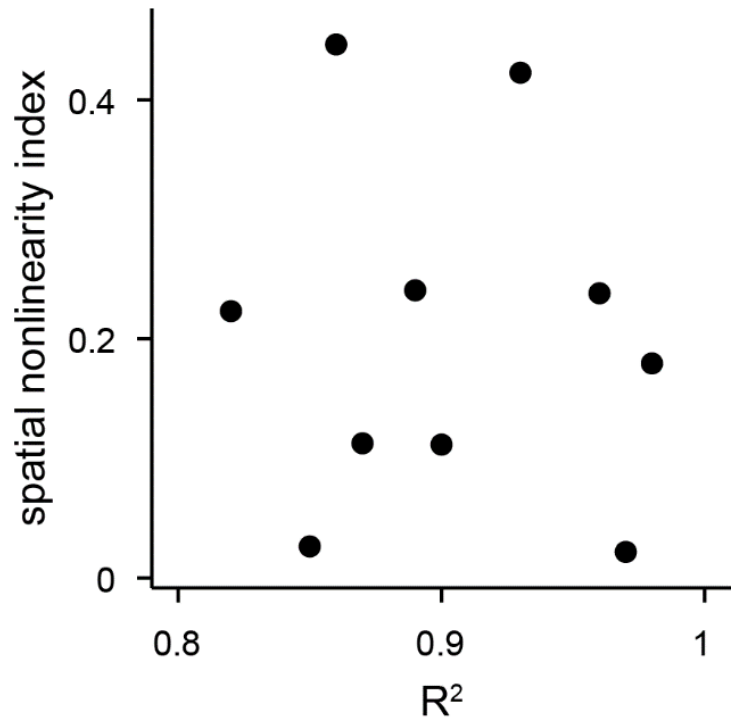

Figure 6.3. Comparison of the prediction performance and the nonlinearities. A. Comparison to the output nonlinearity index. No significant correlation was observed $(\mathrm{r}=-0.51, p=0.15, \mathrm{n}=10)$. B. Comparison to the spatial nonlinearity index, again no significant correlation could be observed $(\mathrm{r}=-0.08, p=0.82, \mathrm{n}=10)$.

\subsubsection{Is the prediction performance related to the general response characteristics?}

Further, we were curious whether the performance of the model is related to the response properties observed in bipolar cells (e.g. fast vs. slow). Indeed, we found a significant correlation between the response latencies under a full-field light stimulation and the performance of the model. Both, for the latency of the filter (Fig.6.4A, $\mathrm{r}=-0.79, p=0.01, \mathrm{n}=10$ ) as well as the latency of the full-field light steps (Fig.6.4B, $\mathrm{r}=-0.76, p=0.01, \mathrm{n}=10$ ), we found that faster bipolar cells exhibited higher performance values than slower bipolar cells. However, for the latency during a center spot activation, we did not find a connection $(\mathrm{r}=-0.46, p=0.36, \mathrm{n}=6)$. Thus, we were curious, whether this relationship is mediated through a surround that influences the speed of the bipolar cells. Yet, we did not find a clear connection between the prediction performance and the surround strength (Fig.6.4C, $\mathrm{r}=0.5, p=0.14, \mathrm{n}=10$ ). To test further whether the surround influences the prediction performance of the model, one would have to compare the performance under a center and full-field white noise stimulation. In Figure 6.4E-F, we show the results of one cell that was stimulated with full-field white noise (E) and center white noise (F). For this particular cell, we did not find a difference in the model performance (85\% vs. 84\%). Further experiments with center and full-field white noise stimulation would be needed to study 
whether the surround could explain the slight differences in the performance of the linearnonlinear model.

Another explanation for the relationship between the latency and the prediction performance could be, that the latency itself is a quality measure of the cell (i.e. the reliable the cell the faster the cell). We tested this idea by comparing the trial-to-trial deviation (computed as the standard-deviation over trials) (see Fig.6.4D) with the latency $(r=0.5, p=0.13, \mathrm{n}=10)$. Here, we find that slow cells can show low and higher trial-to-trial variation (red circles). Thus, we do not believe that the latency is affected by the quality of the cell. The other response characteristics e.g. duration of the response, diameter etc. were not connected to the model performance.

A

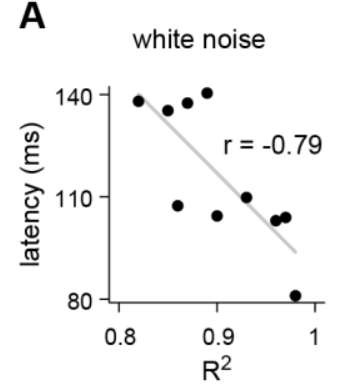

E

\section{B}

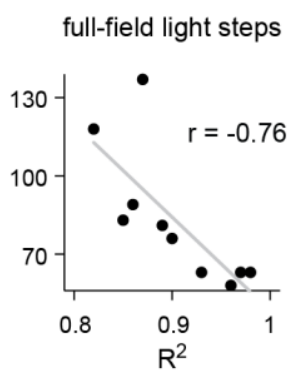

C

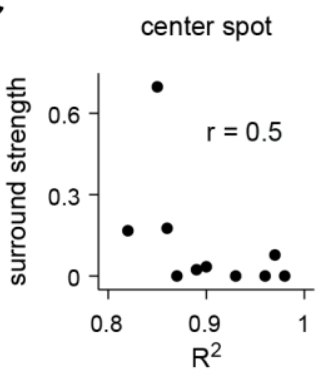

D

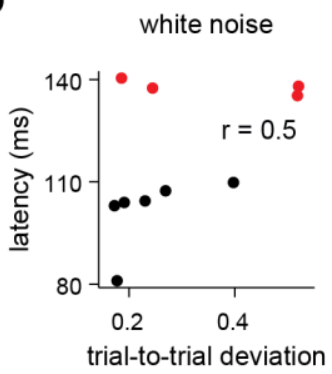

cell 1
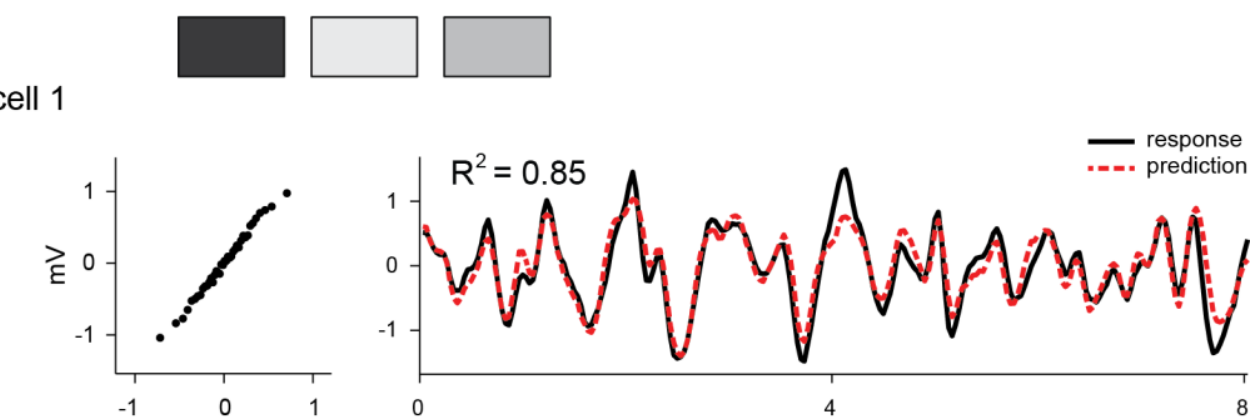

$\mathbf{F}$

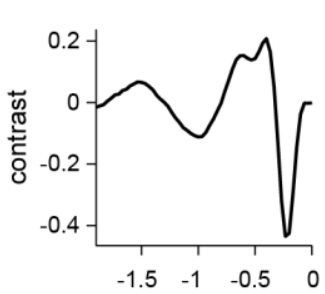

cell 1
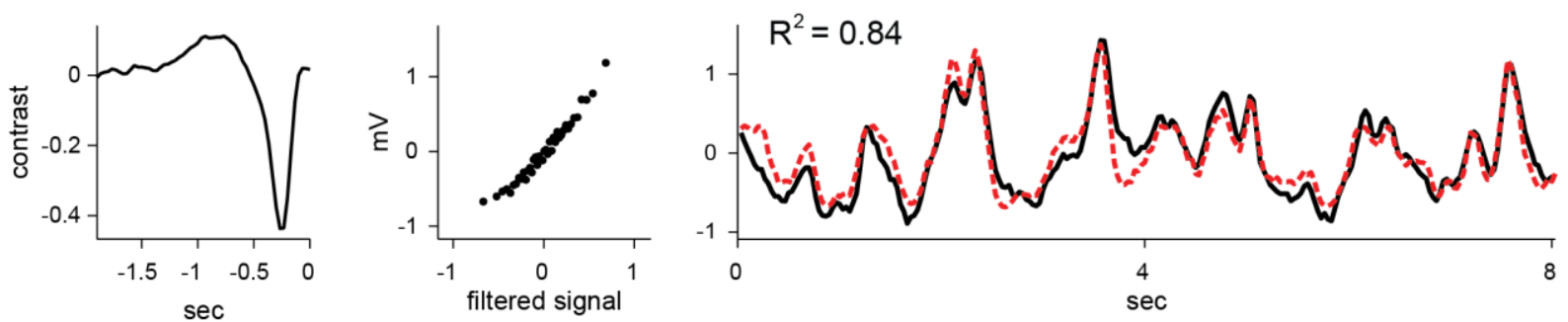

Figure 6.4. Comparison of the prediction performance and the general response properties. A. The latency of the white noise filter was negatively correlated with the prediction performance $(r=-0.79, p=0.01, n=10)$. B. Further, the latency of the full-field light steps was negatively correlated with the prediction performance $(r=-0.76$, $p=0.01, \mathrm{n}=10)$. C. The surround strength measured with the spot stimulus was not correlated to the prediction performance $(\mathrm{r}=0.5, p=0.14, \mathrm{n}=10)$. $\mathrm{D}$. The trial-to-trial variation was not correlated to the latency of the white-noise filter $(\mathrm{r}=0.5, p=0.13, \mathrm{n}=10)$. E. Liner-nonlinear model and prediction of an 
example cell under full-field Gaussian white noise (prediction matches by $85 \%$ ). F. Liner-nonlinear model and prediction of the same cell under center Gaussian white noise (prediction matches by $84 \%$ ).

To conclude, we found high prediction performance values of the linear-nonlinear model under a uniform light stimulation. The close match between predicted and real responses of the cells points out that the responses to a spatially uniform stimulus can be accurately described through a model that assumed a linear integration in space and time. Because the stimulus is spatially uniform, the spatial integration describes mainly the combination of center and surround signals. Here, we think that the influence of the surround is small and a linear combination of signals in the surround might happen. Further, there seems to be no obvious influence of temporal nonlinearities like the account for history-dependent voltage changes such as a refractory-period, bursts or adaptation as proposed for retinal ganglion cells (Pillow et al., 2005; Pillow et al., 2008).

\subsection{Encoding of spatially structured light stimuli}

In the previous subchapter, we have looked at the performance of the linear-nonlinear model with uniform stimuli. Uniform stimuli are simplified artificial stimuli. To study complex artificial stimuli, we added spatial structure. For retinal ganglion cells, the model fails to predict responses under such artificial spatially structured stimuli. Thus, we were curious whether for bipolar cells the linear-nonlinear model predicts the responses accurately.

Our spatially structured stimulus was a binary white noise sequence (Fig.6.5). Thus, the screen was subdivided into small squares that randomly changed to either black or white (see also methods 3.5). The implementation of the linear-nonlinear model was similar to the previously described uniform light stimuli, except that now, our stimulus has a spatial and temporal dimension (see methods 3.7.2).

In this subchapter, we first describe the prediction performance of the model for two single bipolar cells as well as the population data (section 6.2.1). Then, we investigate the relation of the model performance to the nonlinearities (section 6.2.2). Thereby, we focus on the spatial integration. Finally, we examine the relationship of the model performance to the general response characteristics (section 6.2.3). 


\subsubsection{Prediction performance with spatially structured light stimuli}

In Figure 6.5A and $\mathrm{C}$, we see the two stages of the linear-nonlinear model (i.e. filter and output function) for two bipolar cells. In contrast to the uniform stimulus, the filter is built of a spatial (receptive field) and temporal component. The bipolar cell in Figure 6.5A showed a linear output transformation, the bipolar cell in Figure 6.5C exhibited a nonlinear transformation. From the built model, we then predicted the responses to a new stimulus segments (red dashed traces in Fig.6.5B and D). The predicted responses matched the real responses for cell 1 by $\sim 70 \%$ (black and red trace, Fig.6.5B). However, the predicted and real responses mismatched for cell 2 (black and red trace, $\mathrm{R}^{2}=40 \%$, Fig.6.5D). 


\section{A}

Cell 1

\section{LN-model}

1. filter

2. output function
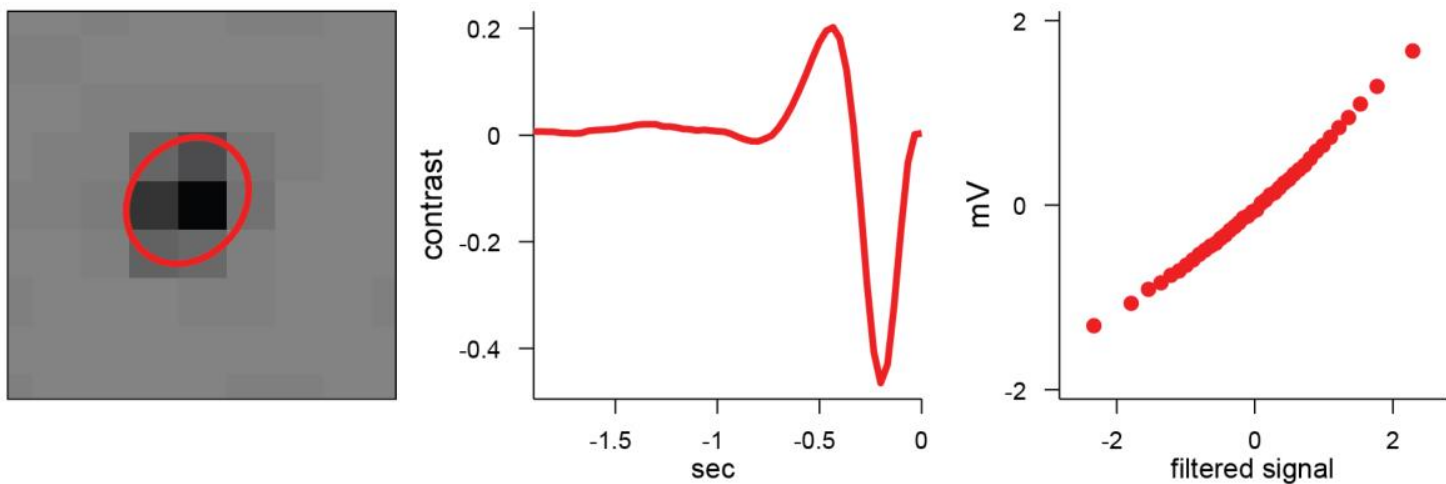

B

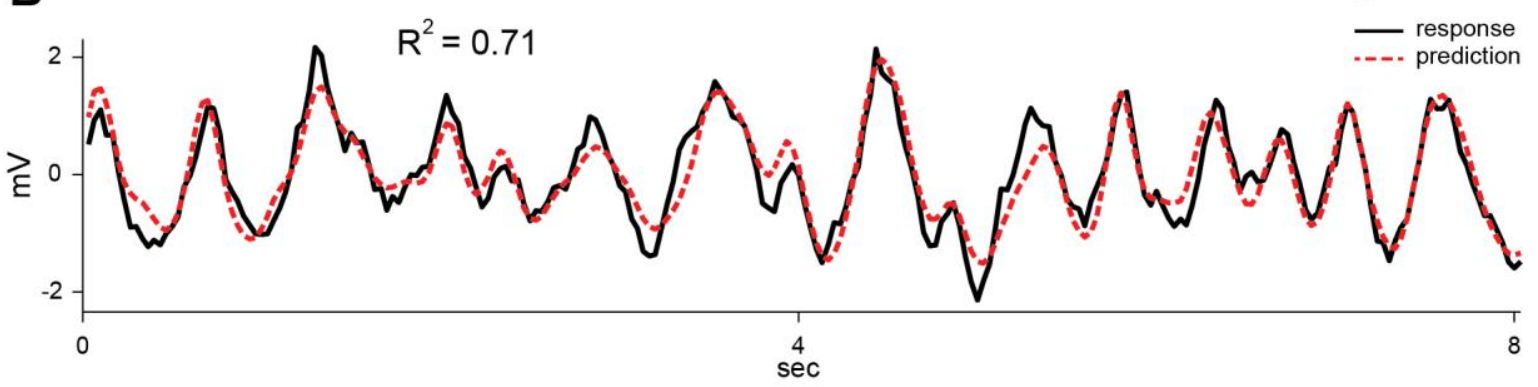

C

Cell 2

1. filter

LN-model

2. output function
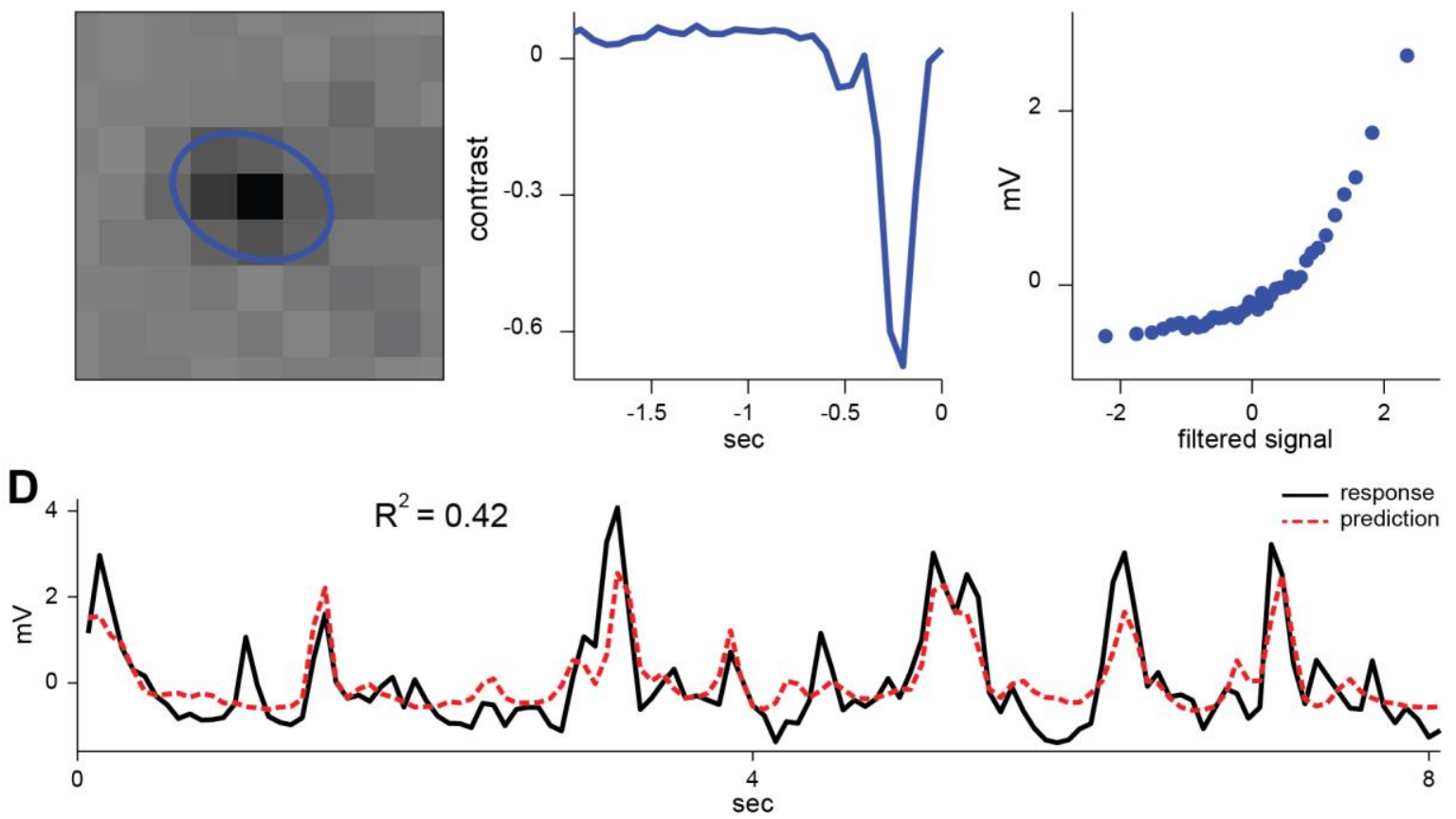

Figure 6.5. Linear-nonlinear model (LN-model) with binary white noise. A. Two stages of the linear-nonlinear model (LN-model): 1. spatial and temporal filter and 2. output function for example cell one. The cell showed a linear output function. B. Response (black) and prediction (red dashed) trace to a binary white noise 
sequence. The average performance was $\sim 71 \%$. C. Same as in A for an example cell with a nonlinear output function. D. Same as in B with an average prediction performance of $42 \%$.

The population distribution of the prediction performance is shown in Figure 6.6 and varied between $36-82 \%(\mathrm{n}=22)$. The distribution is much broader than for the previously documented uniform stimulus of $82-97 \%$. Furthermore, with the stimulus with spatial structure, only for $\sim 30 \%$ of the data the prediction is higher than $70 \%$. Thus, the linear-nonlinear model is much less accurate in predicting the responses to a spatially structured stimulus.

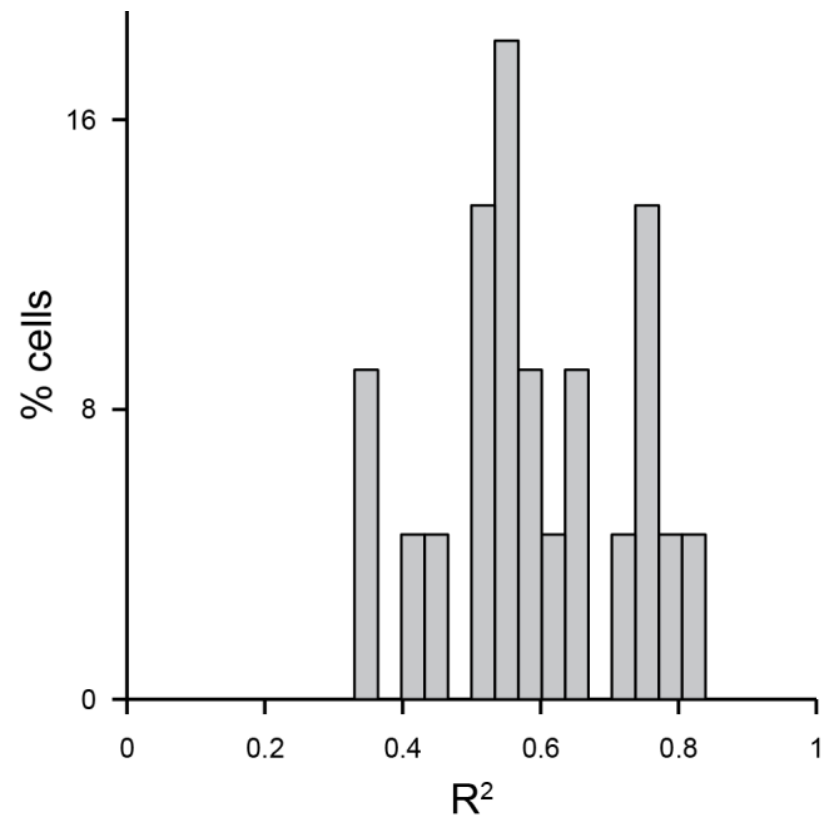

Figure 6.6. Population distribution of the prediction performance with spatial stimulus. The range of the prediction performance $\left(\mathrm{R}^{2}\right)$ is between $36-82 \%$ with 22 bipolar cells.

\subsubsection{Is the prediction performance related to the observed nonlinearities?}

In a next step, we investigated the reasons for the failure of the linear-nonlinear model for predicting stimuli with spatial structure. Thereby, we focused on one theoretically deduced hypothesis, elucidated in the following paragraph.

In Figure 6.7, we illustrated a simplified bipolar cell with a receptive field filter and an output function. The contrast is quantified as the Weber contrast between -1 and 1 (see corresponding red values in Fig.6.7). If we present to this cell a gray stimulus, the filtered stimulus signal is zero (receptive field filter multiplied to gray stimulus). In the second stage, the filtered output is passed through the output function to get the predicted membrane potential 
and here, again, the predicted value is zero (see red circle in Fig. 6.7). There are further stimulus combinations that produce the exact same prediction output of zero. For example, the stimulus arrangement with half black and half white inside the receptive field or with four quarters produces output values of zero. Thus, for all these different stimulus combination, the linearnonlinear model, predicts a membrane potential change of zero. However, do bipolar cells truly not respond to such stimuli? Moreover, if some cells respond to such stimuli, is this the reason for the failure of the model?

spatial filter
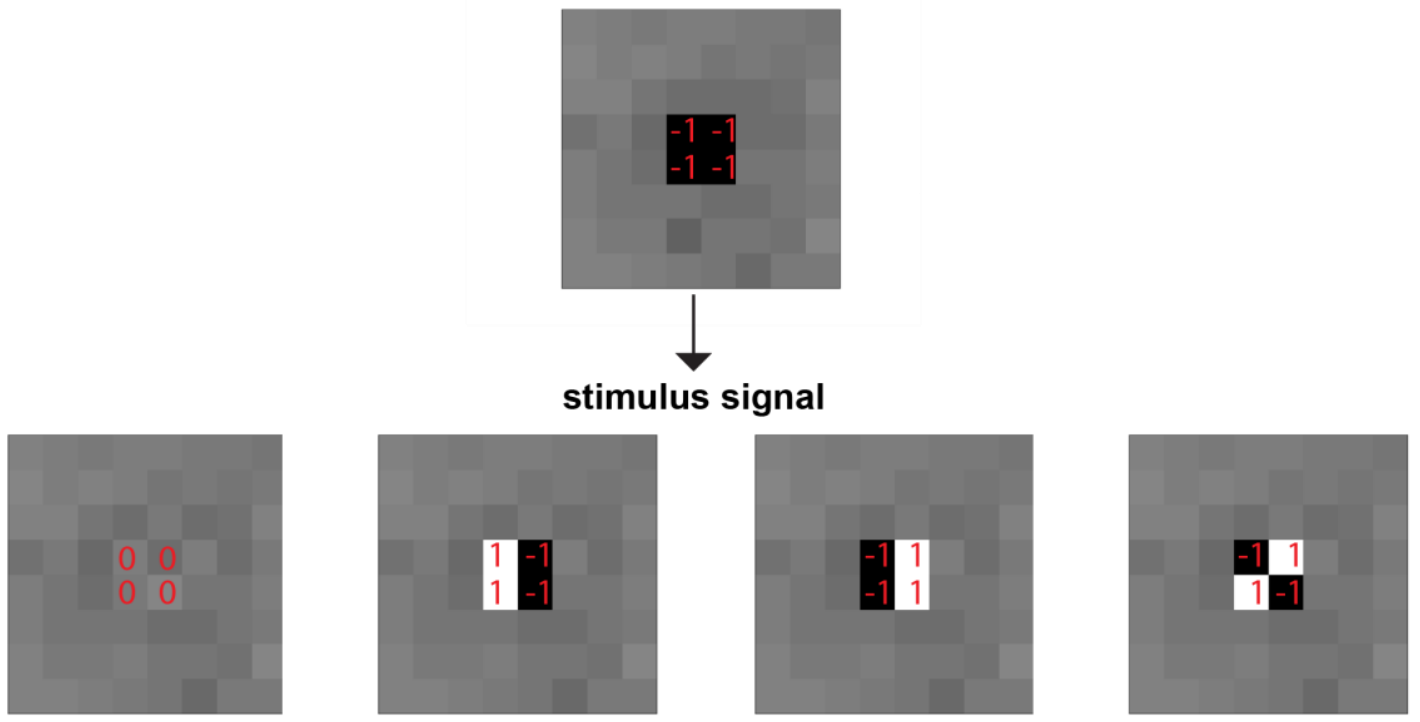

\section{filtered stimulus signal $=0$}

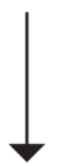

output function

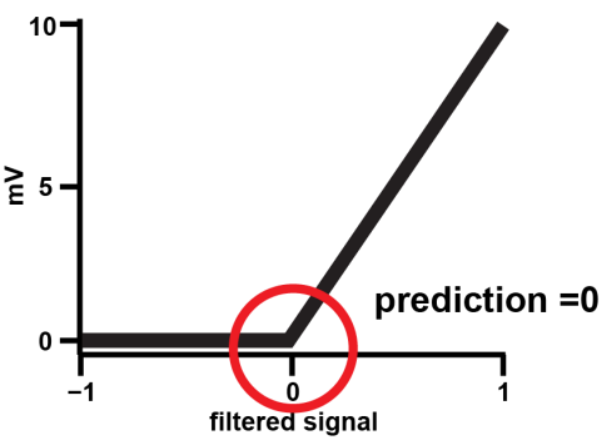

Figure 6.7 Nonlinear spatial integration leads to wrong prediction outputs.

We have seen in chapter 5 that some bipolar cells indeed responded to stimuli with spatial arrangements similar to the once shown in Figure 6.7. Hence, in a next step, we investigated how 
the performance outcome of the linear-nonlinear model is connected to the nonlinear spatial integration. We hypothesized that bipolar cells exhibiting nonlinear spatial integration, also show lower prediction performance and bipolar cells performing linear integration show higher prediction performance. In Figure 6.8A, we illustrate the responses to the reversing grating for the same cells as previously described in Figure 6.5. For the spatially linear bipolar cell (cell 1, red traces) we found a good prediction performance (illustrated in Fig.6.5B); while, for the spatially nonlinear bipolar cell (cell 2, blue traces) the prediction performance was low (illustrated in Fig.6.5D). In Figure 6.8B, we show the relationship on a population level, by depicting the performance of the linear-nonlinear model against the spatial nonlinear index. The two are strongly negatively correlated ( $\mathrm{r}=-0.71, p=0.01, \mathrm{n}=13$, colored points mark the two cells). Thus, as we hypothesized, we found that spatially linear bipolar cells exhibited higher prediction performance than spatially nonlinear bipolar cells.

A

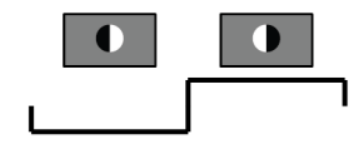

Cell 1

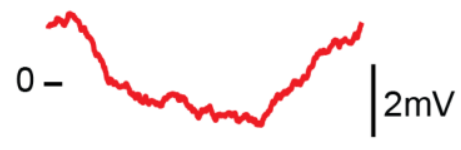

Cell 2

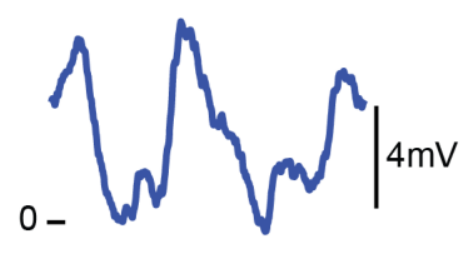

B

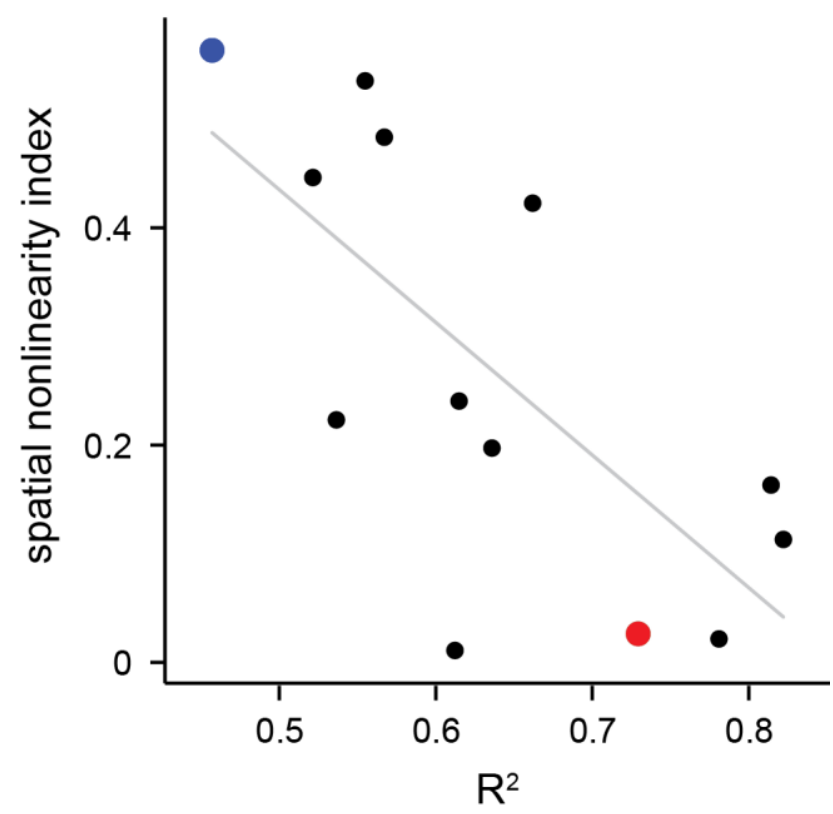

Figure 6.8. Comparison of the prediction performance to the spatial integration. A. Response to the reversing grating of the same cells as in Figure 6.5. Blue cell showed a low prediction performance of 44\%, the red cell showed a high prediction performance of $71 \%$. B. Population comparison between the spatial nonlinearity index and the prediction performance $\mathrm{R}^{2}$. The negative correlation is significant $(\mathrm{r}=-0.71, p=0.01, \mathrm{n}=13)$.

A further way to study the effect of nonlinear spatial integration on the model performance is by inspecting the stimulus-response function. We have seen in Figure 6.7 that the model leads to a filtered output of zero for different spatial arrangements. Further, as we have seen in chapter 5 , some bipolar cells responded to such different spatial arrangements. Thus, in 
the output function, one would expect to see a higher range of membrane potential changes ( $\mathrm{y}$ axis) at filtered output values of around 0 (x-axis), for nonlinear compared to linear cells. Figure 6.9, shows the output function and the prediction performance for a linear (A) and nonlinear (B) cell. Here, the output function not simply contains the averaged responses (red dots, this is how we have plotted the response function until now), but all membrane potential changes (black dots). If we look at the range of the black dots, one might see that for the linear cell in Figure 6.9A, the distribution in the $\mathrm{y}$-axis (membrane potential change in $\mathrm{mV}$ ) is less broad and similar over all x-values. For the nonlinear cell in Figure 6.9B however, we see much broader distribution in the membrane potential of the black dots starting from $\mathrm{x}$-values at around 0 . At filtered values of $\sim 0$ (x-axis), we compared the range of the membrane potential changes (y-axis) (see the pink dots in Fig.6.9A\&B). We indeed found that the higher the range of membrane potential changes at around 0 , the lower also the prediction performance (Fig.6.9C, $\mathrm{r}=-0.51, p=0.02, \mathrm{n}=22$, red dots are the example cells). These findings further indicate that under nonlinear spatial integration, stimuli with different spatial arrangements are assigned to mismatched filtered output values of zero. The mismatched filtered output values lead to higher membrane potential ranges per average response and therefore to a higher error. 
A

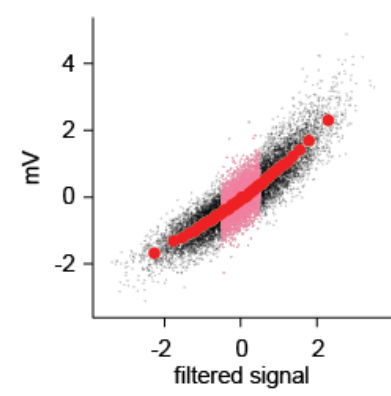

B

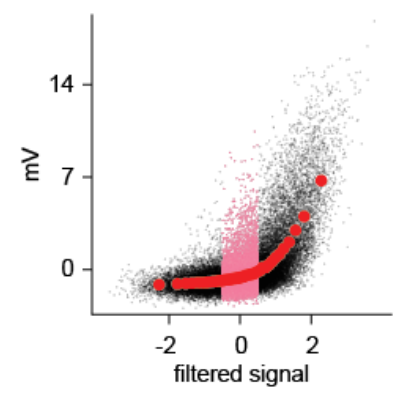

cell 1

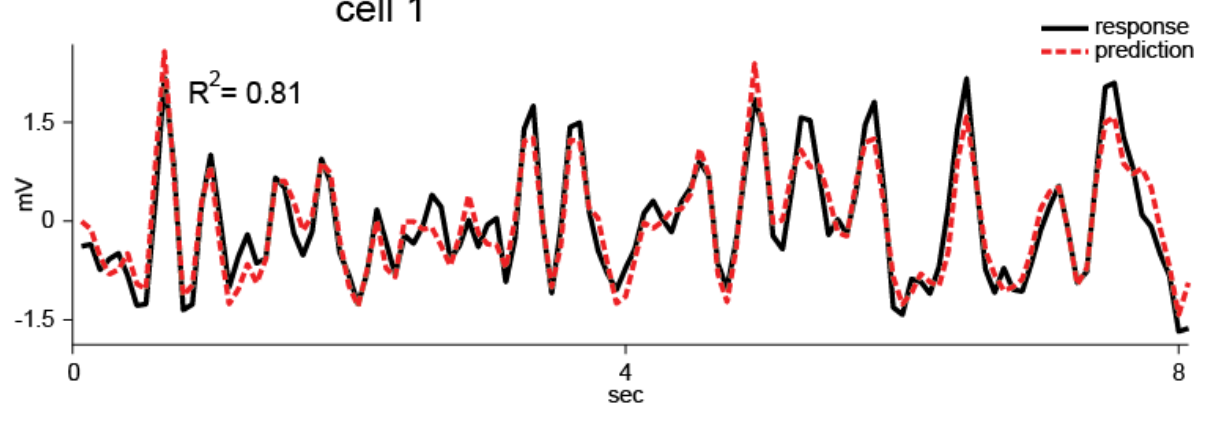

cell 2
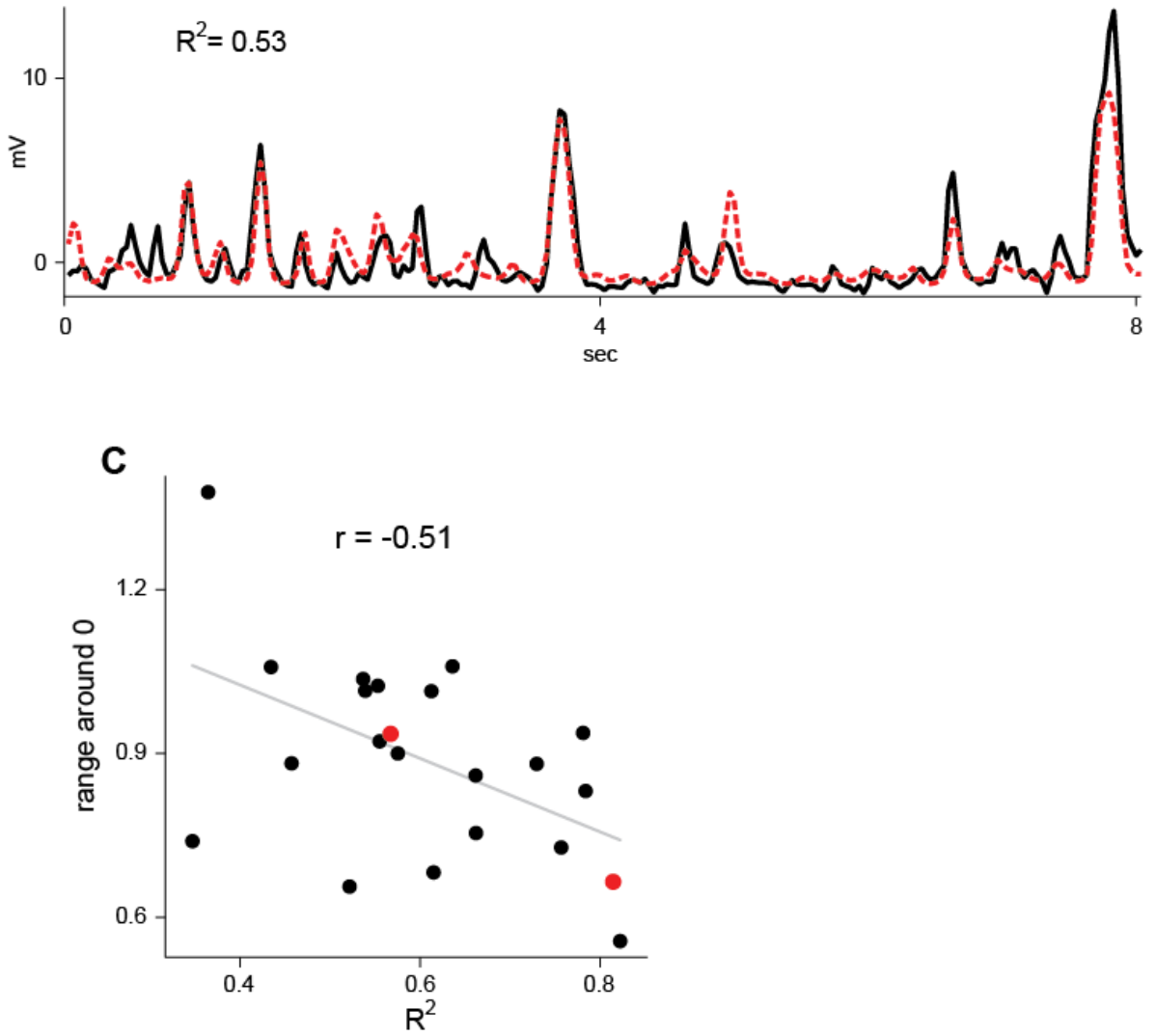

Figure 6.9. The membrane potential range at around zero in the output function. A. Example cell with a linear output function and a good prediction. The pink shaded dots at a filter output of $\sim 0$ show the range of the membrane potential on the y-axis. B. Example cell with a nonlinear output function and a worse prediction. Here the distribution of the black dots are much broader along the y-axis, as one can see from the pink shaded dots for the filtered signal of $\sim 0$. C. Population data, where the prediction performance computed as $\mathrm{R}^{2}$ is plotted against the range in the membrane potential around the filtered output of zero. We observed a significant negative correlation $(\mathrm{r}=-0.51, p=0.02, \mathrm{n}=22)$. 


\subsubsection{Is the prediction performance related to the general response characteristics?}

We further investigated whether the model performance to spatially inhomogeneous stimuli is connected to the described general response characteristics in chapter 4 . We found a significant connection between the sustained-transient index of the bipolar cells under a center spot stimulation and the performance of the model. The more sustained the response of a bipolar cell was, the higher the prediction performance (Fig.6.10A, r=0.83, $p=0.02, \mathrm{n}=7$ ). Further, we found that the faster the bipolar cell responded to the center spot stimulus, the higher again the prediction performance (Fig.6.10C, $\mathrm{r}=-0.92, p=0.01, \mathrm{n}=7$ ). These relationships were expected, given the significant correlations between the nonlinear spatial integration and the temporal properties of the center spot (see subchapter 5.2.4). Similar to there, the clear relationships were only present under a center stimulation. When comparing the performance of the model to the sustained-transient index of the large spot the relationship was less pronounced (Fig.6.10B; $\mathrm{r}=0.75, p=0.05, \mathrm{n}=7)$ and for the full-field light steps the relationship was absent $(\mathrm{r}=0.32, p=0.2$, $\mathrm{n}=17)$. Further, the relationship of the model performance with the latency completely disappeared when comparing it to the large spot (Fig.6.10D; $\mathrm{r}=0, p=0.85, \mathrm{n}=7$ ) or full-field light steps $(\mathrm{r}=-0.23, p=0.38, \mathrm{n}=17)$.

To finalize, for the other response characteristics e.g. diameter size, surround strength, oscillations, we did not find a connection. For example, both cells in Figure 6.5 have a small receptive field, yet one of them shows a high prediction performance the other a low performance.

These results again highlight that the temporal characteristics of the receptive field center are crucial to understand the origin of spatial nonlinear information integration, which is here the main reason for the failure of the model. Further, the results point out that other response characteristics (e.g. surround strength) are not majorly involved in the failure of the linearnonlinear model under spatially structured stimuli. To conclude, our results show that the linearnonlinear model can predict responses of bipolar cells accurately if they integrate their inputs linearly. The linear bipolar cells are the sustained and fast cells. The model on the other hand fails to predict responses of bipolar cells that integrate their inputs nonlinearly. The nonlinear bipolar cells are the transient and slow cells. 
A

center spot

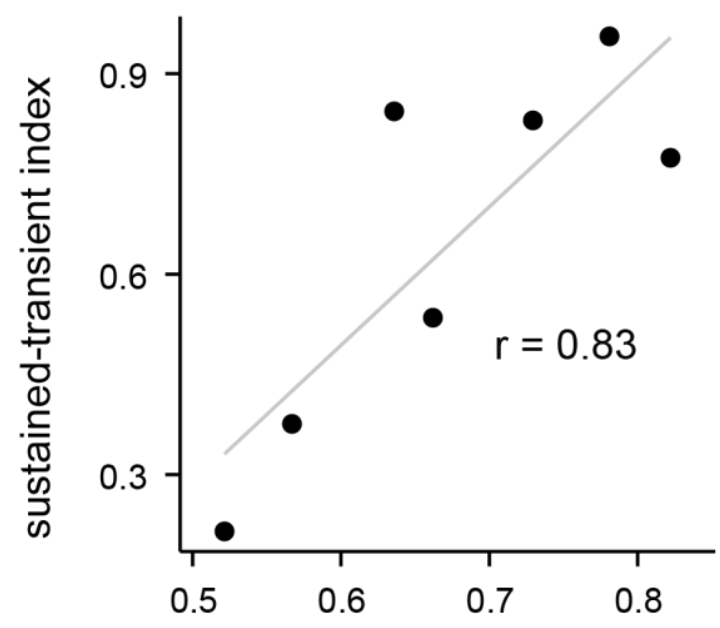

C

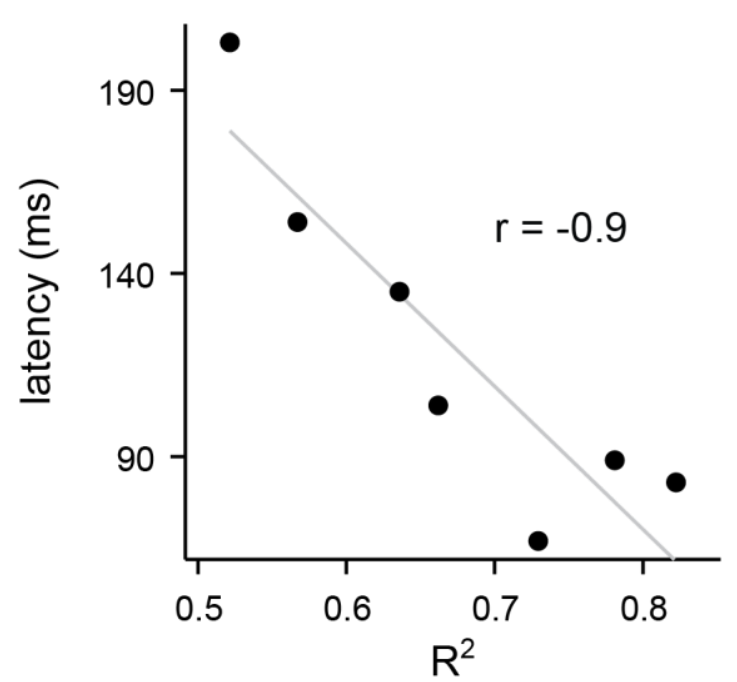

B

\section{large spot}

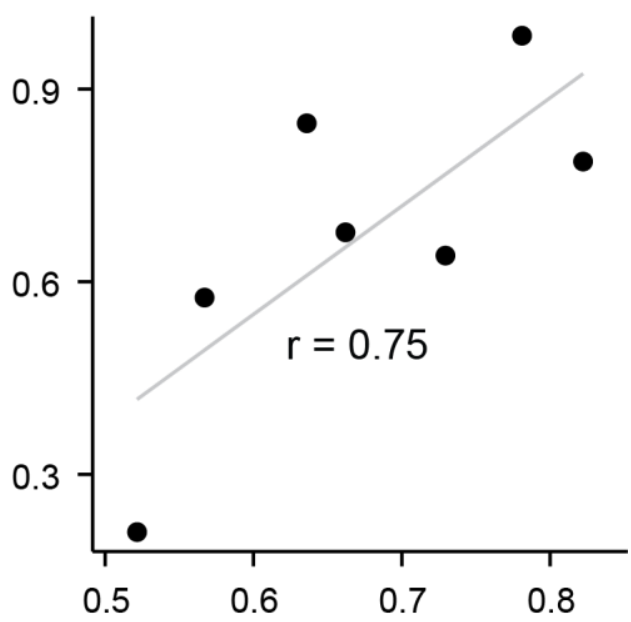

D

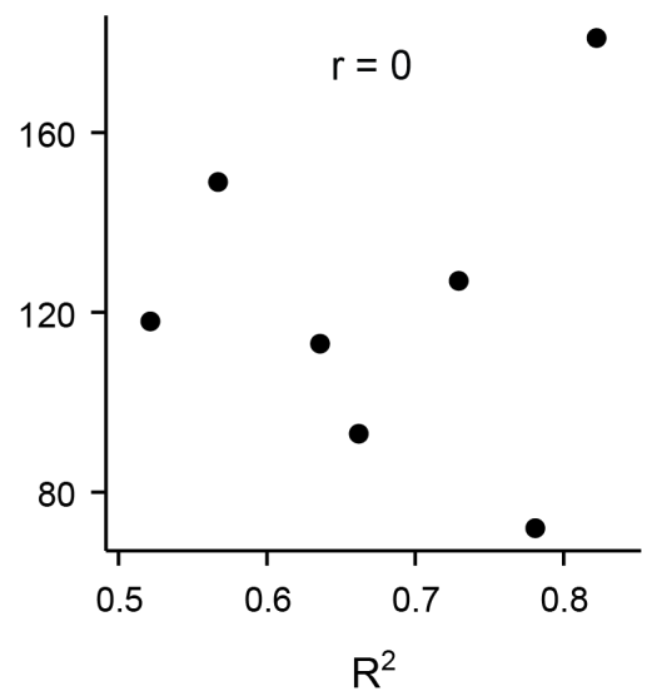

Figure 6.10. Performance outcome correlated with center and surround temporal properties. A. Clear negative correlation of the prediction performance with the sustained-transient index under a center spot stimulation $(\mathrm{r}=-0.83$, $p=0.02, \mathrm{n}=7)$. B. Under the large spot the relationship was less pronounced $(\mathrm{r}=0.75, p=0.05, \mathrm{n}=7)$. C. Negative correlation between the response latency and the model performance under a center spot stimulation $(\mathrm{r}=-0.9, p=0.01, \mathrm{n}=7)$. D. The relationship was not visible under a large spot light stimulation $(\mathrm{r}=0, p=0.85, \mathrm{n}=7)$. 


\subsection{Encoding of natural light stimuli}

In the previous chapters, we have studied how bipolar cells encode artificial light stimuli. We have seen that for uniform light stimuli the linear-nonlinear model predicts responses very accurately. However, for stimuli with spatial structure, the model failed when bipolar cells showed nonlinear spatial integration. In this subchapter, we were curious about the encoding of natural light stimuli. To our knowledge, natural light stimuli have not yet been used for bipolar cells.

Therefore, we first studied whether bipolar cells responded to natural light stimuli (section 6.3.1). Then, we investigated whether we can predict their responses with the linearnonlinear model (section 6.3.2). Further, we studied the role of spatial integration. Here, we were curious whether bipolar cells also integrate information nonlinearly under natural light stimuli or if nonlinear spatial integration is an "artifact" of artificial stimuli (section 6.3.3). Finally, we have a brief glance at the role of adaptation under natural stimuli (section 6.3.4).

\subsubsection{Do bipolar cells respond to natural light stimuli?}

First, we inspected, how bipolar cells responded to natural light stimuli. To do so, we showed natural movies to the bipolar cells (see example frames of two movies in Figure 6.11). The natural movies were selected from the standardized "CatCam" database (Betsch et al., 2004) (see methods 3.7.3). We showed between 1-5 different natural movies to one bipolar cell and repeated each movie $\sim 10$ times.

In Figure 6.11A, we see the receptive field location of a bipolar cell for two different natural movies. Further, we see the responses of the cell to the single trials (colored traces) as well as the average (black traces). The cell responded very reliably over the different trials for both movies. Further, by comparing the response traces between movie 1 and movie 2, one can clearly see that the cell responded differently to the two movies. In Figure 6.11B, we see the receptive field location as well as the responses to the same two movies for another bipolar cell. Again, for both movies, the bipolar cell responded very accurately over trials. In addition, when comparing the traces of the two bipolar cells for the same movie (e.g. compare Fig.6.11A and B for movie 1); it becomes clearly visible that the different bipolar cells responded differently to the same movie. 
A

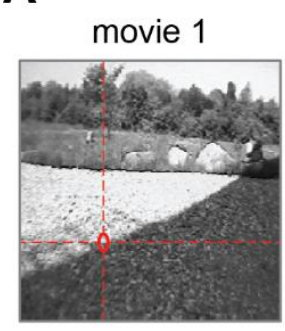

movie 2
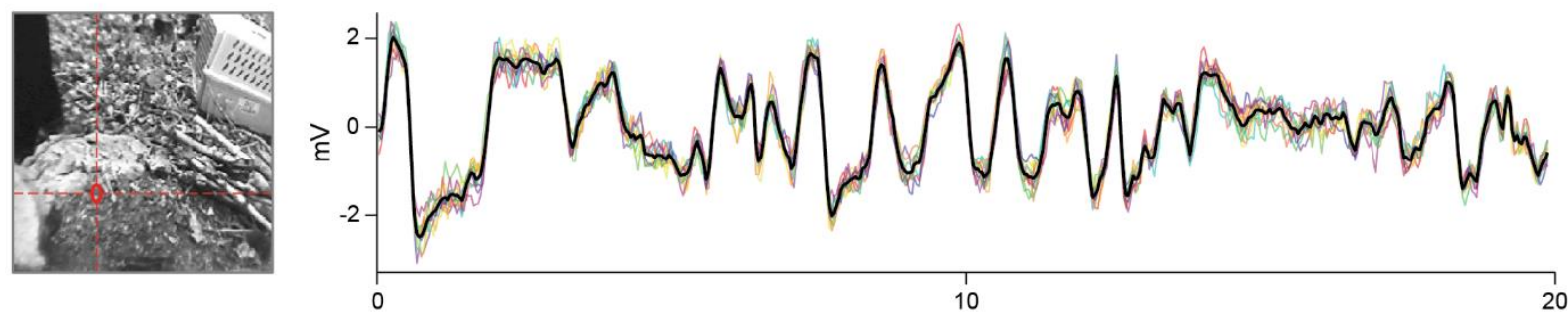

cell 1
B

movie 1

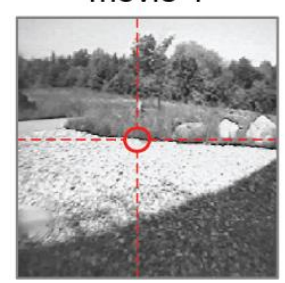

movie 2

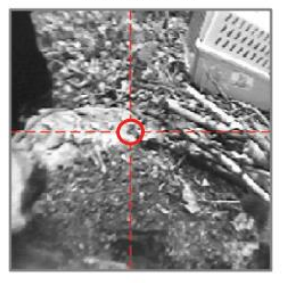

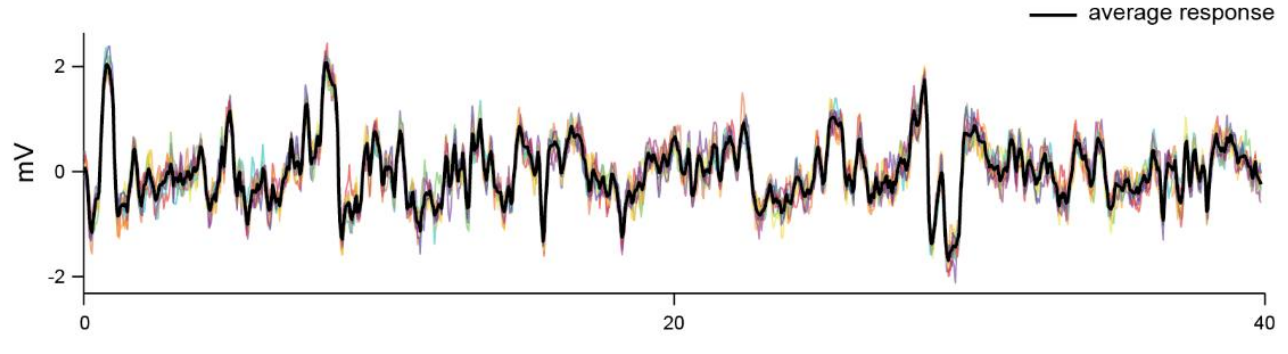

cell 2
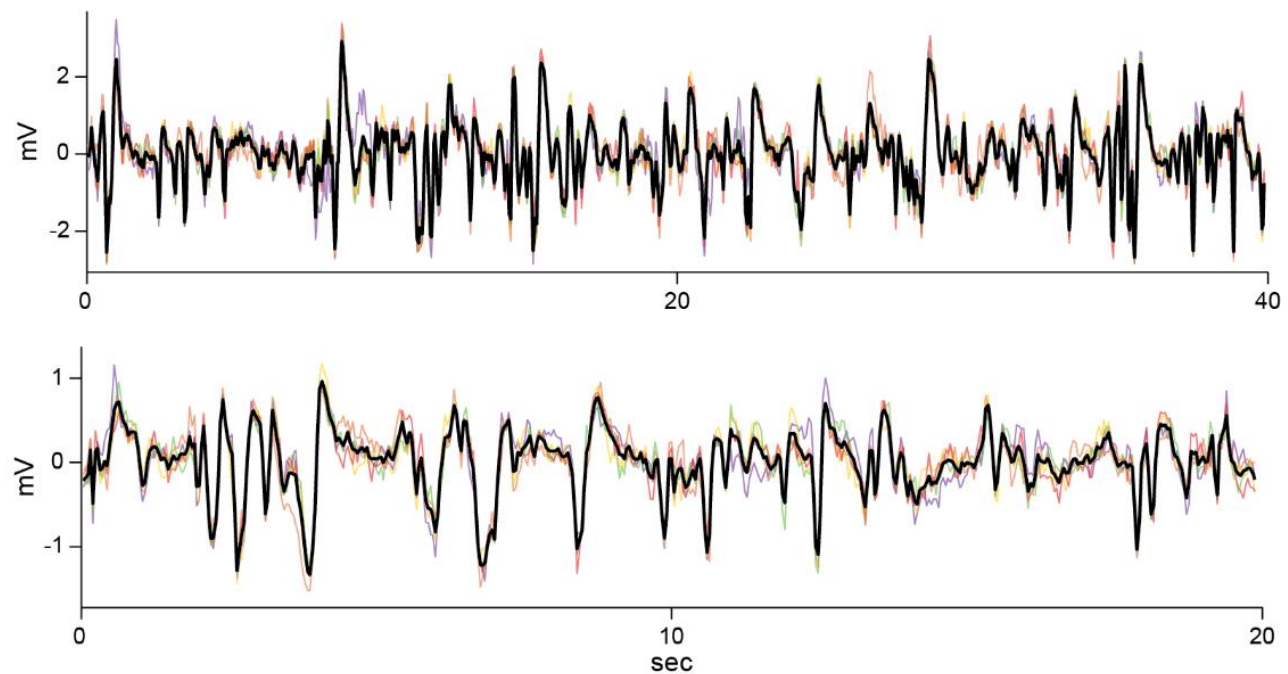

Figure 6.11. Response traces to natural movies. A. Receptive field location and response traces of example cell 1 to two different natural movies. Red circle represents the receptive field location of the cell on two movie frames. The colorful response traces are the responses to the single trials, the black trace is the average response B. Receptive field location and response traces of another example cell to the same natural movies as in A.

In total, we recorded natural movies for 8 bipolar cells. For all bipolar cells, we found clear responses to the natural movies. To quantify how reliable the bipolar cells responded to the natural movies, we computed the trial-to-trial deviation (mean standard-deviation over trials). The trial-to-trial deviation varied between $0.13-0.5$ (mean $=0.26, \mathrm{SD}=0.1)$. Thus, very similar to the uniform light stimulus with a trial-to-trial deviation between $0.17-0.5$ (mean=0.3, $\mathrm{SD}=0.14$ ). From these results, we concluded that bipolar cells clearly responded reliably to natural movies. Further, the same bipolar cell seemed to respond differently to different natural movies and 
different bipolar cells seemed to respond differently to the same movie. This diversity in encoding makes it interesting to investigate with the linear-nonlinear model.

\subsubsection{Prediction performance with natural light stimuli}

To predict bipolar cell responses to natural stimuli, the two stages of the linear-nonlinear model (i.e. filter and stimulus-response function) are first built from the responses to the binary white noise stimulus (see methods). Then, the filter is compared to the natural movie sequence and the output is passed through the stimulus-response function to predict the membrane potential change.

In Figure 6.12A and D, we see the two stages of the linear-nonlinear model (i.e. filter and stimulus-response function) for two bipolar cells. The filter is built of a spatial (receptive field) and temporal component. Further, we see the location of the receptive field on a natural movie frame. From the built model, we then predicted the responses to the natural movie (dashed traces in Fig.6.12C and F). The predicted and real response for cell 1 only matched by $\sim 62 \%$ (black and red traces, Fig.6.12C). The predicted response matched the real response for cell 2 by $\sim 88 \%$ (black and blue traces, Fig.6.12F).

From 8 recorded bipolar cells, only 4 showed reliable responses to the binary white noise stimulus (see methods 3.7.2 for quality criteria). In total, we recorded around 10 natural movies from the 4 bipolar cells. Our population distribution varied between 44-91\% for the 10 movies. From a first glance, the distribution looks broader than for the uniform stimulus with $82-97 \%$ and more similar to the stimulus with spatial structure of 36-82\%. However, more bipolar cells are needed to compare the distributions. 
A

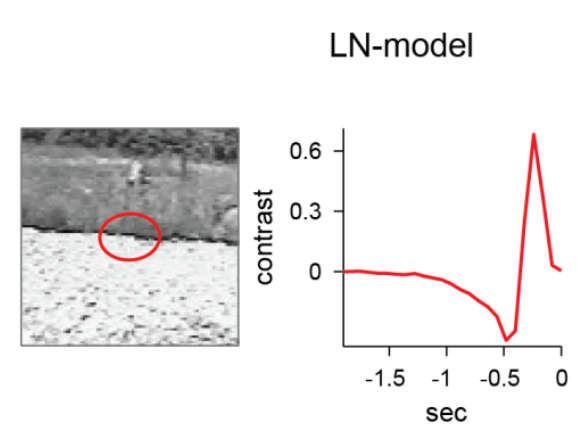

cell 1

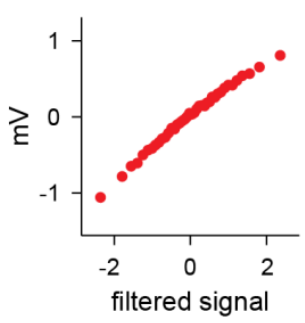

B

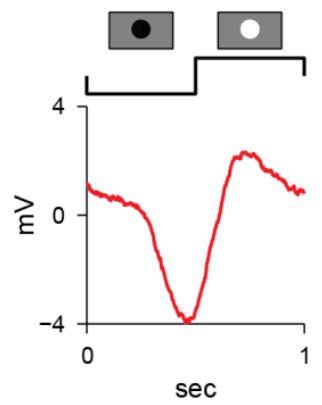

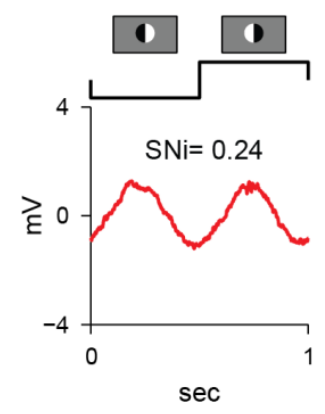

C

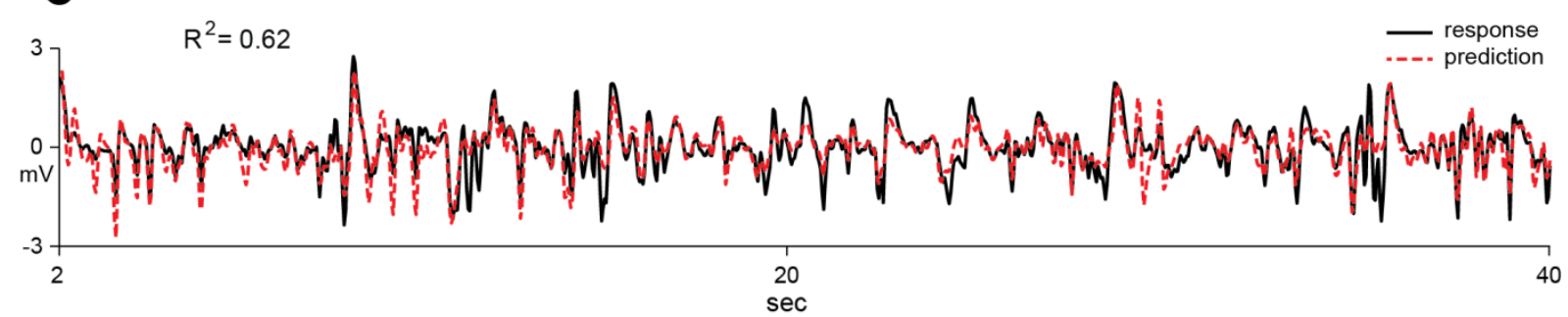

D

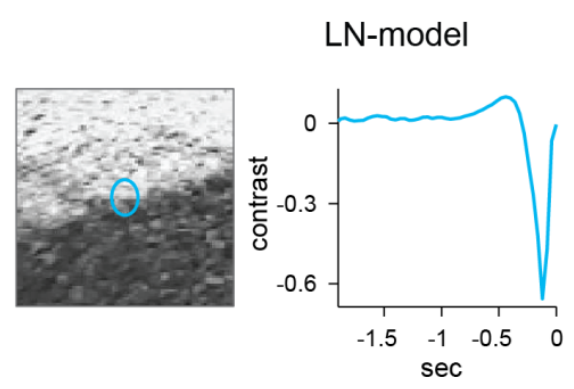

cell 2

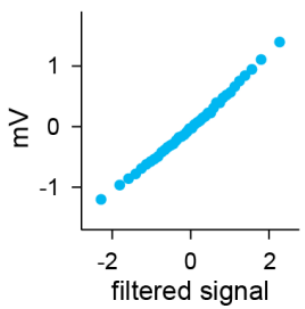

E

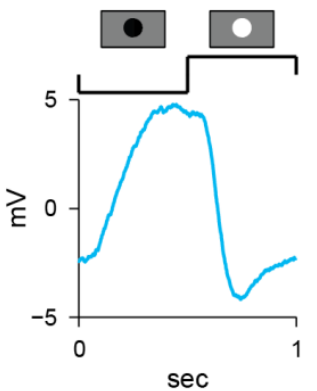

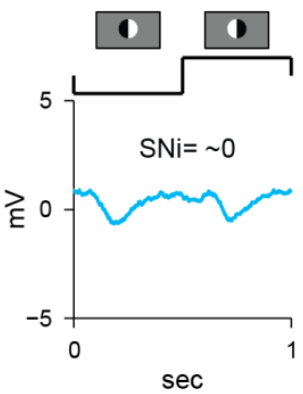

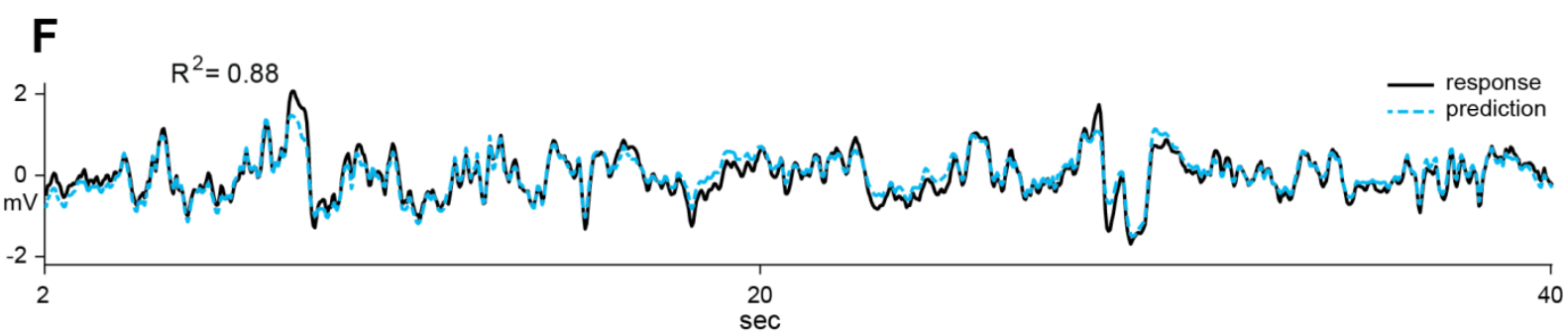

Figure 6.12. Prediction of natural movies' responses with the linear-nonlinear model. A. The two stages of the LNmodel with the filter and nonlinearity. The example cell is an ON cell with a saturation in the nonlinearity. B. Traces of cell 1 to the black-white spot and the grating. The cell responded to the grating and showed a spatial nonlinearity index ( $\mathrm{SNi}$ ) of $\sim 0.24$. C. Original (black) and predicted (dashed red line) response traces of the cell to the natural movie, the accuracy of the prediction is $\sim 62 \%$. D. LN-model for another example cell. The cell is an OFF cell with a linear stimulus-response transformation. E. Response traces to the black-white and grating spot. The cell strongly reduced the response to the grating and showed a SNi of almost 0. F. Predicted (blue dashed line) and original (black line) response traces to the natural movie, the match is $\sim 88 \%$. 


\subsubsection{Is the prediction performance connected to the observed nonlinearities?}

In a next step, we investigated whether the performance outcome of the linear-nonlinear model is connected to the nonlinear spatial integration. For the bipolar cell with a lower prediction performance of $62 \%$ in Figure 6.12C, we found a more nonlinear response to the spatial grating with a spatial nonlinearity index ( $\mathrm{SNi}$ ) of 0.24 (see Fig.6.12B). For the cell with an accurate prediction of $88 \%$ in Figure $6.12 \mathrm{~F}$, we found a linear response to the spatial grating with a SNi of 0 (see Fig.6.12E). In Figure 6.13, we show the relationship on a population level; by depicting the performance of the linear-nonlinear model against the spatial nonlinear index (red dots are the example cells of Fig.6.12). For those bipolar cells with multiple natural movies, we chose the movie with the highest prediction performance. We observed that the performance and spatial nonlinearity are strongly negatively correlated, spatially linear bipolar cells exhibited higher prediction performance than spatially nonlinear bipolar cells. However, the correlation was not statistically significant $(\mathrm{r}=-0.82, p=0.18, \mathrm{n}=4)$. Clearly, more bipolar cells are needed to confirm the negative correlation. Despite the low number, we have an indication that nonlinear spatial integration is not exclusive to artificial stimuli but also present under natural stimulation and leads to lower prediction performance.

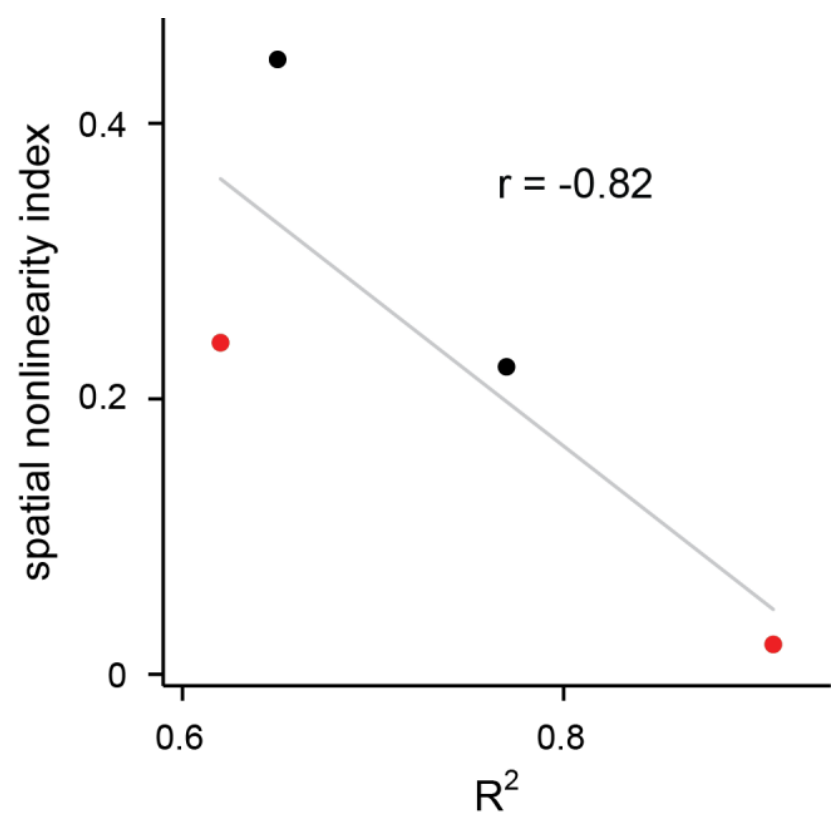

Figure 6.13. Natural movies prediction performance and nonlinear spatial integration. We observed a high correlation coefficient between the performance of the linear-nonlinear model for natural movies and the spatial nonlinearity index $(\mathrm{r}=-0.82, p=0.18, \mathrm{n}=4)$. Yet, the correlation is not statistically significant. 
Another interesting observation is illustrated in Figure 6.14. Here, we show the real and predicted responses for another spatially nonlinear bipolar cell for two movies. For movie 1 (Fig.6.14A), the prediction performance is around 65\%, while for movie 2, the performance is $\sim 44 \%$ (Fig.6.14C). A decrease in the quality of the membrane potential could not explain this difference, for both movies, the membrane potential was very stable. We were therefore wondering, whether the spatial structure that the bipolar cell was seeing inside its receptive field, was different for the two movies. For example, it could be that in movie 1, the bipolar cell was exposed more often to uniform contrast, which would lead to a higher prediction performance, because the nonlinear spatial integration has no effect (similar to the uniform contrast stimulus in subchapter 6.1). In movie 2 , on the other hand, the bipolar cell would be exposed to spatial structure and therefore, the effect of nonlinear spatial integration is leading to a worse prediction performance. To study this idea, we quantified the spatial structure of the natural movies. To do so, we calculated the standard deviation of the contrast inside a bipolar cell's receptive field. For example, the standard deviation for the frame of movie 1 in Figure $6.14 \mathrm{~B}$ is very low $(\sim 0)$, because the cell mainly sees homogenous black. For the movie frame in Figure 6.14D, however, the standard deviation is higher $(\sim 0.4)$, because the cell is exposed to dark and bright regions. For this particular cell, we indeed found that on average the standard deviation was lower in the movie 1 compared to movie 2 ( 0.12 vs. 0.18$)$. This observation could be an indication that for natural movies, the outcome of the prediction performance depends on the spatial structure a cell is exposed to inside the receptive field. The hypothesis is that the more homogenous the light signal inside the receptive field the better the prediction for a nonlinear cell and vice versa.

For the remaining three cells, we could not study this question. One cell was spatially linear and therefore showed good performance in all natural movies, independent of the seen spatial structure. For another cell, the difference in prediction performance between the movies was most likely due to lower quality of the membrane potential signal. For the last cell, we only recorded one movie. To inspect the influence of the spatial structure inside a natural movie, more experiments, with stable recording for different natural movies, are needed. 
A
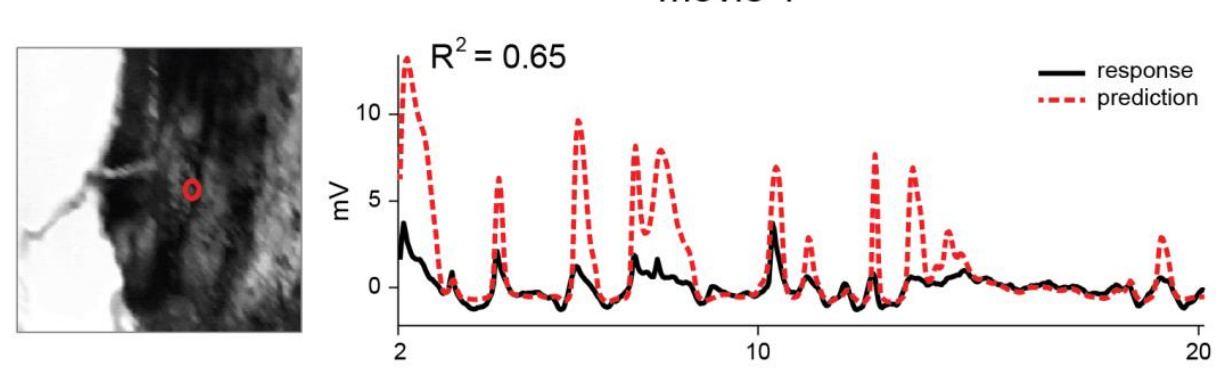

C
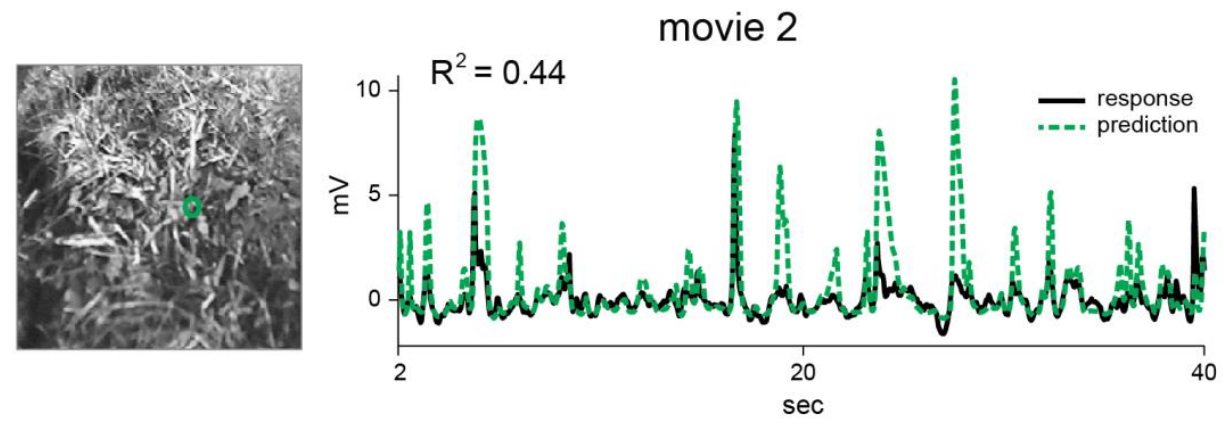

B spatial structure $=\sim 0$

D spatial structure $=0.4$

Figure 6.14. Prediction performance of two natural movies for the same cell. A. Location of the receptive field (red circle) on the movie 1 and the original (black) and predicted (dashed red line) response traces to the natural movie. The accuracy of the prediction is $\sim 65 \%$. B. Zoom into the receptive field location and the spatial structure the cell sees. Here, the standard deviation of the contrast inside the receptive field is $\sim 0$. C. Location of the receptive field (green circle) of the same cell as in A on the movie 2 and the original (black) and predicted (dashed green line) response traces to the natural movie. The accuracy of the prediction is $\sim 44 \%$. D. For the example frame, the spatial structure has a standard deviation of $\sim 0.4$.

To finalize, we compared the performance of the linear-nonlinear model for natural movies and binary white noise. Curiously, we found that the performance was for all cells better with natural movies compared to the binary white noise stimulus (see Fig.6.15, dashed line is unity line). The artificial stimulus has more spatial structure than the natural stimuli and we think that this explains the lower performance of the artificial compared to the natural stimulus. 


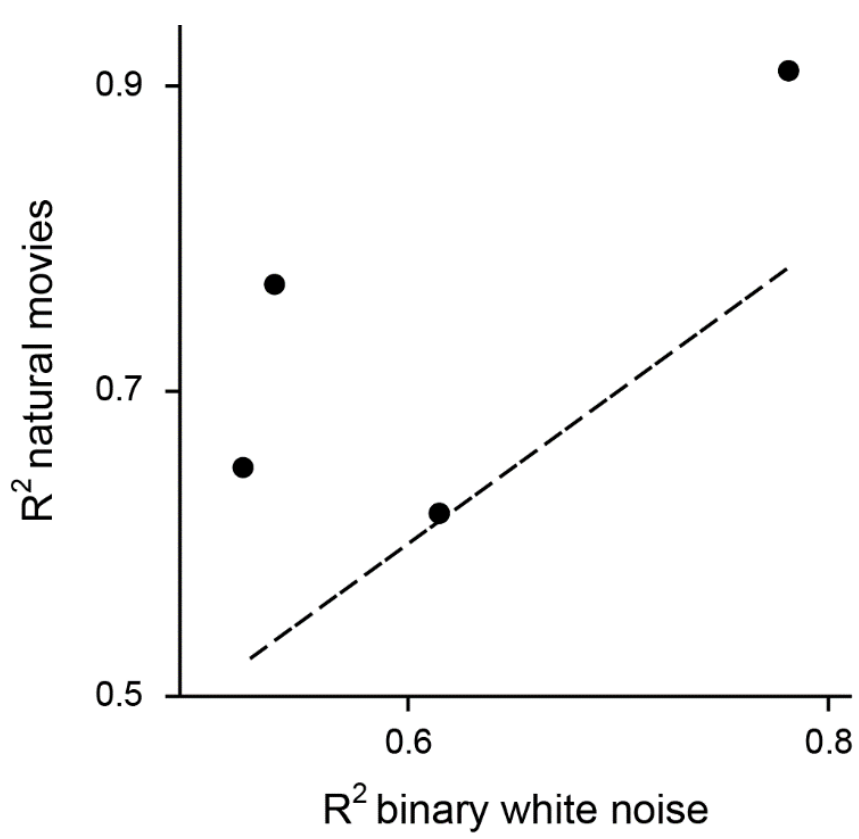

Figure 6.15. Prediction performance with natural movies and binary white noise. For all four bipolar cells, the prediction performance under the natural movie was higher than under the binary white noise stimulation. Black dashed line is the unity line (i.e. where the points would be if the prediction performance under the natural movie were identical to the binary white noise stimulus).

\subsubsection{Adaptation under natural light stimulation}

When carefully inspecting Figure 6.12 and 6.14, one might realize that for example in Figure 6.14A, the predicted amplitude is much higher than the amplitude of the real response. For the bipolar cell in Figure 6.12C, on the other hand, the predicted amplitude is closer to the amplitude of the real response. Yet, both cells exhibit a similar performance outcome $\sim 60 \%$. We were wondering, what makes the linear-nonlinear model to sometimes overestimate the amplitude?

Natural stimuli might change the adaptation process of the cells, because of longer exposures to scenes with fixed contrast (Heitman et al., 2016). Further, adaptation can change the filter and the output nonlinearity (Baccus and Meister, 2002; Zaghloul et al., 2005). Thus, we wonder whether under natural stimuli, adaptation causes the overestimation of the model by changing the output function. To test this idea, we compared the stimulus-response function from the binary white noise, where we assumed no adaptation (because of fast contrast changes), to the stimulus-response function of the natural movies, where adaptation might happen (see methods 3.7.3). In Figure 6.16A and C, we plotted the output functions for two different bipolar cells. Both cells have the same performance output, but the predicted amplitude for cell 1 is overestimated (Fig.6.16B). We indeed found, that for cell 1, the stimulus-response function with natural movies changed to a smaller range (blue dots, -0.5 to +1 , Figure $6.16 \mathrm{~A}$ ) compared to the 
response function with the binary white noise (red dots, range -0.5 to +3 , Fig.6.16A). For the other cell, the stimulus-response functions stayed very similar (compare blue and red dots in Fig.6.16C). Thus, we think that adaptation leads to an overestimation in the model by reducing the range of the membrane potential fluctuations. For example, cell 1 only showed a maximum membrane potential change of $1 \mathrm{mV}$ for natural movies compared to the $3 \mathrm{mV}$ for binary white noise. When predicting the responses to the natural movie of $1 \mathrm{mV}$ with the response function of the binary white noise of $3 \mathrm{mV}$, we overestimate the membrane potential. For both, binary white noise as well as natural movie, the cell was recorded stably at an average potential of $\sim-57 \mathrm{mV}$.

A way to account for the adaptation under natural stimuli is by predicting the bipolar cell responses from the stimulus-response function of the natural movies (Heitman et al., 2016). Here, the stimulus statistics (e.g. temporal dynamics) for the model as well as for the predicted sequence are similar. In Figure 6.16 B and D, we show the prediction with the stimulus-response function of the natural movie as blue traces. As one can see for cell 1, the amplitude becomes closer to the original trace. The explained variance however stayed similar between the prediction with the binary white noise $(65 \%)$ and natural movie response function $(63 \%)$. For cell 2 , the prediction amplitude does not change (62\% vs. 65\%). For the particular cell 1, adaptation may influence mainly the amplitude, however not the spatial integration or temporal properties and therefore does not influence the general performance of the model. However, further experiments, to increase our low number of bipolar cells, would help to study the effect of adaptation on the model performance. 
A

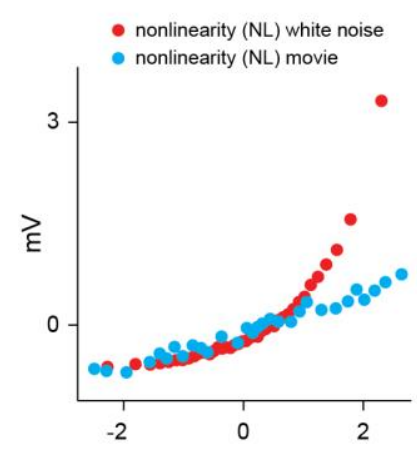

C

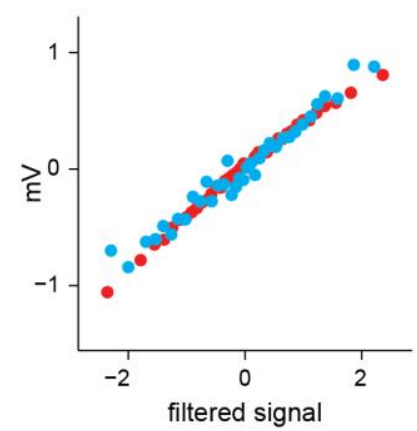

B

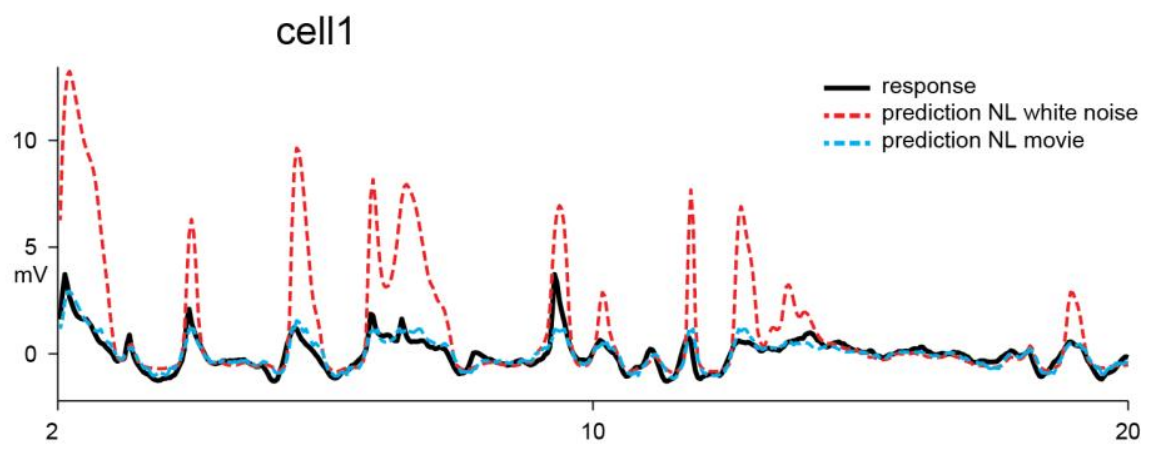

cell2

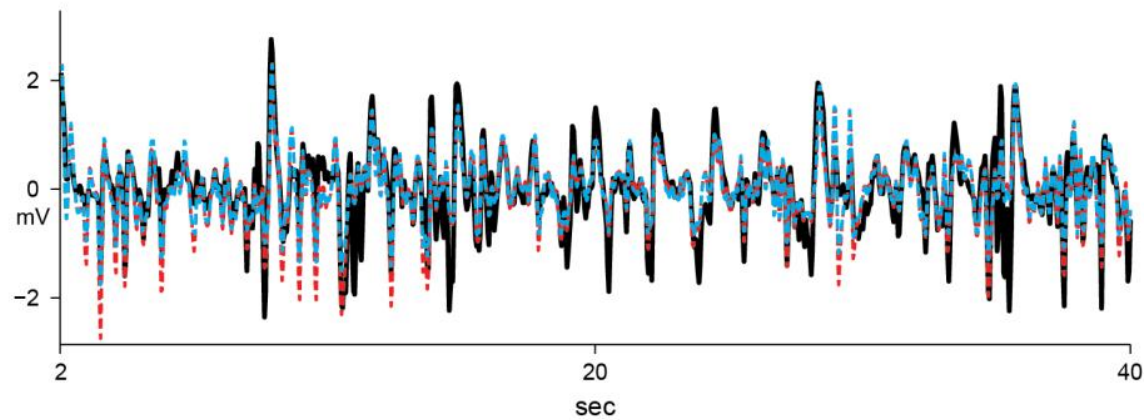

Figure 6.16. Linear-nonlinear model fitted with natural movies and white noise. A. Nonlinear output function (NL) fitted with white noise (red) and natural movie (blue). B. Prediction traces of the same cell fitted with white noise (red) and natural movie (blue). The performance is very similar (65\% vs. 63\%). C. Same as in A for another example cell, the output function fitted with white noise is very similar to the output function fitted with natural movie. D. Same as in B, the prediction performance with white noise and natural movie is very similar $62 \%$ vs. $65 \%$. 


\section{Discussion and Outlook}

\subsection{Summary}

Retinal ganglion cells come in about 30 different types (Baden et al., 2016). The diverse cell types evolve from photoreceptors to ganglion cells. However, the contribution of the different intermediate cell types is not well understood. Bipolar cells represent pivotal elements by connecting the outer and inner layers of the retina.

It has been proposed that at the heart of the observed response diversity in retinal ganglion cells lie nonlinear signal transformations from bipolar cells to ganglion cells (Gollisch, 2013; Gollisch and Meister, 2010; Roska and Meister, 2014). Yet, how the nonlinear signal transformations manifest themselves in bipolar cells is not understood. Nonlinear signal transformation can occur at two stages in a neuron: at the inputs, also termed spatial nonlinearity and at the output, termed output nonlinearity.

Traditionally, bipolar cells are assumed to be linear, both in the integration of their inputs as well as at their output at the soma. Thus, in this thesis, we investigated the rumor of linear signal encoding in bipolar cells. To do so, we worked on three main goals:

- Goal 1: Establishing a general characterization of bipolar cells

- Goal 2: Assessing nonlinearities in bipolar cells

- Goal 3: Predicting bipolar cells' responses with the linear-nonlinear model

In our first goal, we studied bipolar cells' responses to classical light stimuli. We found that bipolar cells did not respond homogenously to light but showed a diversity in their responses. We observed bipolar cells with different receptive field sizes, with and without inhibitory surround as well as with diverse temporal response profiles. For example, we found transient and sustained 
bipolar cells as well as bipolar cells with a plateau after an initial peak and bipolar cells with oscillations. Thus, bipolar cells do not simply passively transmit the photoreceptor signals to the ganglion cells, like an extended axon, but process and split the input light signal into parallel channels. Characterizing bipolar cells' responses was an important first step before studying their nonlinearities.

In the second goal of the thesis, we investigated two types of nonlinearities in bipolar cells: output nonlinearity and spatial nonlinearity. Curiously, for both types, we observed linear and nonlinear response properties. Bipolar cells showed nonlinear output transformations when probing them with simple flashing spot stimuli and flickering contrast stimuli. Thus, bipolar cells do not simply transmit light in a pixel-by-pixel fashion to the ganglion cells, but nonlinearly enhance or reduce certain aspects of the visual scene. Furthermore, we found that bipolar cells themselves already integrate their inputs nonlinearly. Thus, they do not simply sum dark and light patches inside their receptive fields to gray, rather, they are sensitive to fine spatial structures and report them to downstream neurons. Furthermore, we found that the two types of nonlinearities are related among each other. The more nonlinear the output function the more nonlinear the spatial integration. This finding indicates that the spatial nonlinearity strongly influences the output nonlinearity. In addition, we observed that the nonlinear properties are cell type specific. We observed that the more transient the bipolar cell responded to light, the more nonlinear the output and spatial integration properties.

In the third goal, we studied the bipolar cell responses with the linear-nonlinear model. The linear-nonlinear model assumes a linear signal transmission from the photoreceptors to the bipolar cells and thus, if this assumption holds, we would expect that the model accurately describes the response of bipolar cells. Yet, we observed that this was not the case. Both artificial and natural stimuli with spatial structure lead to low prediction performance of the model. Curiously, we observed that the failure of the model was related to the degree of nonlinear spatial integration. The more nonlinear the spatial integration in bipolar cells the higher the model failure. The findings suggest that nonlinear signal integration starts already at the level of bipolar cells and that nonlinear computations are crucial properties that mathematical models in the retina have to take into consideration for predicting responses to artificial and natural stimuli. 


\subsection{Role of nonlinearities for encoding visual signals in bipolar cells}

Bipolar cells are broadly assumed to respond to light in a linear fashion; both in their output signals measured at the soma as well as at their inputs, measured through their spatial integration properties. In Figure 7.1A, we picture how bipolar cells are modelled traditionally. They sum light linearly over a single filter and then pass the integrated signal in a linear way to their terminals. Thus, they are pictured as an extended axon of the photoreceptors. If this assumption holds, the linear-nonlinear model would be expected to describe the responses to stimuli with spatial structure accurately. Yet, when introducing spatial structure into the light stimulus, the model failed to reproduce responses for around $70 \%$ of the recorded bipolar cells. Furthermore, we observed that the failure of the model was related to the spatial integration properties. The more nonlinearly the spatial integration in bipolar cells, the higher the model failure. What are the implication of these findings on the linear-nonlinear model applied onto bipolar cells?

\section{Nonlinear models in bipolar cells}

Analog to the Y-type retinal ganglion cells, the findings point out that the presynaptic signals are first nonlinearly transformed before being pooled by the bipolar cell. The nonlinear transformation before the summation assures that presynaptic signals do not cancel. In Figure $7.1 \mathrm{~B}$, we picture how the linear-nonlinear model could be extended to include the observed nonlinear properties. To prevent cancellation, it is important for the model that a nonlinear transformation occurs before the summation of the signal by the bipolar cell. Thus, the input light has to be first nonlinearly transformed, then summed by the bipolar cell and again nonlinearly transformed by cell internal processes before sent to the terminals. The potential mechanism can be viewed as an extension of the $\mathrm{LN}$-model by one additional nonlinear stage, to an LN-LN cascade model. The light input is first sampled by the photoreceptor (1. linear stage) and is passed through a nonlinear function (2. nonlinear stage). Then the signal is weighted and linearly summed by the bipolar cell (3. linear stage) and transformed through the bipolar cells own nonlinear processing stage to generate a response (4. nonlinear stage). Note, our results cannot tell where the exact nonlinear signal transformation stage takes place. To prevent cancellation, it is important for the model that the nonlinear transformation occurs before the summation of the signal by the bipolar cell, yet where exactly is secondary. The nonlinear transformation could for example occur at the level of the photoreceptors or at the bipolar cell dendrites. In the next section 7.3 , we will discuss the potential biological mechanism in detail. 
We have shown that the stronger the bipolar cell nonlinear summation, the higher the failure of the classical LN-model. Thus, we hypothesize that an extension of the LN-model to an LN-LN model would lead to higher prediction performance under stimuli with spatial structure. In the LN-model pictured in Figure 7.1A, the presynaptic signals cancel out toward zero if both dark and white pixels are shown inside the receptive field. Thus, in those cases, the model does not predict a response and fails to describe the bipolar cell. In an LN-LN model as pictured in Figure 7.1B, the presynaptic signals do not cancel out toward zero, because the dark pixels are amplified while the negative effect of the bright pixels is reduced (rectified). Here, the model predicts a response and might therefore correctly describe the bipolar cells. For retinal ganglion cells, the extension of the LN-model with an additional nonlinear transformation before the summation leads to higher prediction performance (Freeman et al., 2015; Liu et al., 2017; Maheswaranathan et al., 2017; Schwartz et al., 2012). Yet, implementing an additional nonlinear transformation comes with challenges. Classically, the problem is approached by subdividing the receptive field into small subunits that first filter and integrate the light stimulus and then transform it nonlinearly. This approach poses diverse questions. For example, how to choose the subunits location and size inside the bipolar cell receptive field? Do the subunits correspond to photoreceptors or to a mixture between horizontal and photoreceptors? Do the subunits even correspond to individual synapses between photoreceptor-horizontal-bipolar cells? To formulate the problem differently, when we stimulate the bipolar cells with pixels of light, do we transform each pixel first nonlinearly before the summation? Or are some neighboring pixels first summed (e.g. by the photoreceptor e.g. due to gap junctions among them) and then nonlinearly transformed? If yes, how to choose which pixels are summed together? For retinal ganglion cells, the problem is approached from different sides, by building for example a model based on anatomical and physiological connectivity maps between bipolar and single retinal ganglion cells (Schwartz et al., 2012). Yet, anatomical and physiological mapping is experimentally challenging and only feasible on a single cell level. An alternative approach to assess the subfields inside the receptive field is individual photoreceptor stimulation (Freeman et al., 2015). This approach could be further combined with an adapted version of our introduced method of spike-triggered non-negative matrix factorization (Liu et al., 2017). As we have shown in Liu et al. (2017), the method of spike-triggered non-negative matrix factorization was successful in locating the bipolar cells inside the retinal ganglion cell receptive fields. Thus, it might be interesting to extend the method to voltage recordings from bipolar cells and try to assess the subfields inside the bipolar cells' receptive fields. A further challenge is to define the shape of the nonlinear transform by the subunits. Is it threshold nonlinear? Threshold quadratic? Does a thresholding include zero hyperpolarization or unequal hyperpolarization and depolarization? Do all subunits exhibit the 
same nonlinear transformation? Here, direct recordings of the output nonlinearities from the neurons presynaptic to bipolar cells might help to clarify which nonlinear transformation should be chosen. Yet, the nonlinear transformation might occur at the bipolar cell dendrites, here, isoresponse measurements could help to assess the nonlinear transformation indirectly at the bipolar cells (Bolinger and Gollisch, 2012).

Note, before trying out new experimental or computational methods, the intuitively simplest option is to try out whether the model performance increases by simply transforming each pixel nonlinearly before the summation inside the bipolar cell. As a nonlinear transformation, the same as the output nonlinearity of the bipolar cell could be chosen.

A

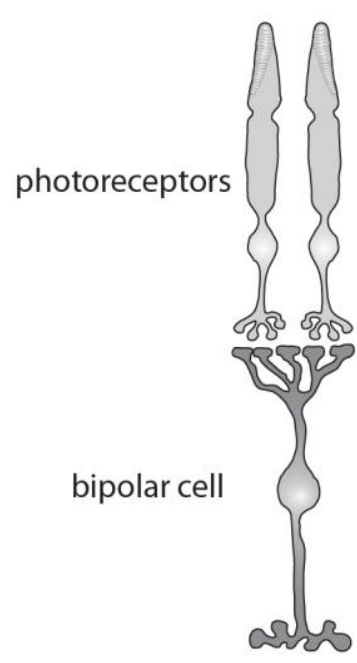

Assumed LN-model

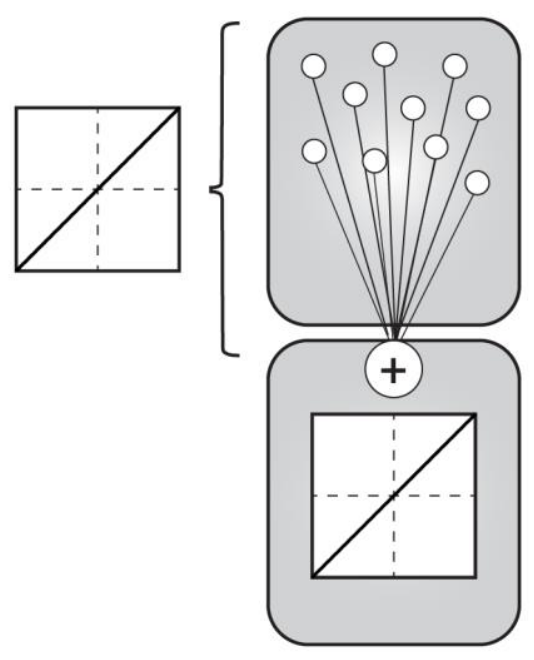

stimulus

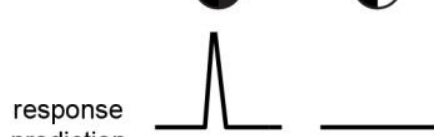

B

Proposed LN-LN-model
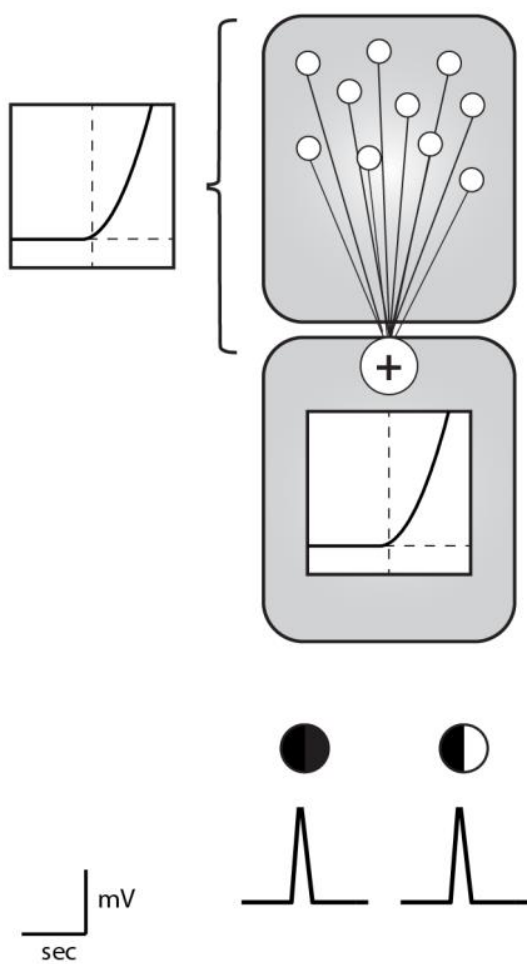

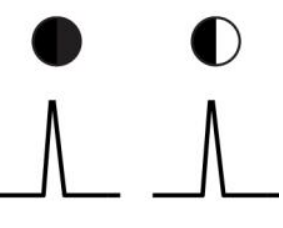

Figure 7.1 Assumed and proposed linear-nonlinear (LN) model. A. Assumed linear-nonlinear model in bipolar cells. Classically bipolar cells are assumed to be linear both in the integration of their inputs as well as in the output transformation at their soma. In such a model, the bipolar cell would not respond to a spot with half black and half white inside the receptive field, because input signals of opposite sign would cancel out to zero B. Proposed extension of the linear-nonlinear model to an LN-LN-model to capture the nonlinear transformations of the inputs before the summation by the bipolar cell. In such a model, the bipolar cell would respond to a spot with half black and half white, because the inputs are first rectified and then summed. 


\section{The degree of spatial structure inside the light stimulus matters!}

For the uniform contrast flicker, the model performance was very high for all bipolar cells. Further, we observed a tendency that the prediction performance is higher under natural stimuli than under artificial stimuli. Might it be the case that the inputs are linearly summed in some conditions and nonlinearly under others? We think that the main difference in the performance comes from the degree of spatial structure inside the stimuli, but the spatial summation stays the same under all type of stimuli. For a uniform contrast sequence, all subunits inside the receptive field are driven in the same direction, either positively or negatively. In such a scenario, no signals can be cancelled and both the LN-model as well as the proposed LN-LNmodel would predict the same response amplitude (Fig.7.1A and B). This is different from an inhomogeneous illumination, here, light and dark regions are presented inside the receptive field. In such a scenario, the $\mathrm{LN}$-model predicts a cancellation of the response, because it assumes that the inputs are changed positively and negatively by the same amount (Fig.7.1A). The LN-LNmodel on the other hand would predict a response. Here, the inputs are first nonlinearly transformed and the negative activation is put to zero while the positive activation from the stimulus is amplified and leads to a response (Fig.7.1B).

For natural movies, we have observed higher prediction performance compared to binary white noise stimulus. Two reasons could explain these findings. First, the degree of spatial structure inside the natural movie seem to influence the prediction performance. For example, our results indicate that bipolar cells can show different prediction performance under different natural movies and movies with higher spatial structure lead to lower performance of the model. Another reason is that the spatial structure the bipolar cells were exposed to under the artificial binary white noise stimuli was generally higher than under natural stimuli. For retinal ganglion cells, it has originally been speculated, that the performance of the LN-model might be lower under natural stimuli than artificial stimuli due to the more frequent presence of dark and light boundaries in natural scenes (Gollisch, 2013; Schwartz and Rieke, 2011). Yet, the amount of dark and light boundaries (spatial structure) seems to depend on the type of natural stimuli presented inside the receptive field. When cells are presented with more homogenous natural stimuli inside their receptive field, they can exhibit higher prediction performance similar to the uniform contrast sequence. Furthermore, bipolar cells exhibit small receptive field compared to ganglion cells, thus it might be, that under natural stimuli, bipolar cells see more homogenous patterns, while under the artificial stimulus (which have size of 22-30 $\mu \mathrm{m}$ ), they are more exposed to spatial structure. Yet, we only have recordings of four good bipolar cells with all three types of stimuli (uniform, binary white noise and natural movies). Thus, to inspect further the relationship 
between the prediction performance and the degree of spatial structure in artificial and natural stimuli, more experiments, with stable recording for different natural movies, are highly desired. In our analysis, we only investigated the average spatial structure of a movie and the binary white noise stimulus. A further way to study the effect of spatial structure on the model performance is by inspecting each sequence of the binary white noise and natural movies separately. One could imagine that some binary white noise or natural movie sequences are more homogenous while others are more heterogeneous. We would hypothesize that the responses to sequences with more spatial structure are more difficult to predict and vice versa. For binary white noise stimuli, an approach to analyze the model performance on single stimulus sequences was introduced by Freeman et al. (2015). They identified sequences for which the prediction of the LN- and LN-LN-model differ most strongly (they termed those "maximally differentiating stimuli”). These sequences could be interpreted as the sequences with higher spatial structure. Yet, the measurement should be combined with the standard deviation or spatial frequency of each sequence to make this conclusion. Here, a similar analysis like Turner and Rieke (2016) would be interesting to perform, they found that the LN-LN-model mainly improves prediction to natural images with higher spatial structure. Another option to study the spatial structure and model performance might be by performing principal components analysis of all stimulus sequences similar to Maheswaranathan et al. (2017).

\section{What about the linear bipolar cells?}

What about the other $30 \%$ of bipolar cells that showed a good performance with the LNmodel? Does the assumed linear transmission from the LN-model correctly describe the findings? We think yes! For spatially linear cells, the linear-nonlinear model correctly describes their responses to all three different type of stimuli (uniform, binary white noise and natural movies). Further, we have observed that the higher the prediction performance the more linear the bipolar cells summed dark and bright light signals over their receptive field. Thus, a linear signal transmission from photoreceptors and horizontal cells to bipolar cells as pictured in Figure 7.1A might be a possible mechanistic explanation for the results.

Our results suggest that dependent on the cell type a different model should be used to predict the bipolar cell response. For example, spatially linear bipolar cells are more sustained and can be predicted by the LN-model without extensions. On the other hand, spatially nonlinear bipolar cells are transient and the LN-LN-model might be used to accurately predict their responses. 


\subsection{Nonlinearities in bipolar cells: why they have not been observed before?}

Traditionally bipolar cells were viewed as linear cells, both in their stimulus-response transformation as well as in their spatial integration. Therefore, in diverse mathematical models (e.g. LN-model) the bipolar cells were approximated as linear neurons that linearly transmit increase and decrease of light. How did the linear rumor become established?

\section{Output nonlinearity}

Diverse previous studies showed approximately linear stimulus-response transformations when recording from bipolar cell somas (Baccus and Meister, 2002; Rieke, 2001; Sakai and Naka, 1987a, b; Sakai et al., 1995; Toyoda, 1974). Here, mainly white-noise stimulation was used, thus the contrast changed randomly. Other studies that also recorded signals from bipolar cell somas reported nonlinear stimulus-response transformations (Burkhardt and Fahey, 1998; Euler and Masland, 2000; Fahey and Burkhardt, 2003). Here, mainly contrast steps were used, thus the contrast increased and decreased from a steady background illumination by fixed amounts. They reported nonlinearities in the form of saturation to very high contrast changes as well as small or almost zero hyperpolarization to contrast decrease (rectification). One might wonder why those studies did not change the view about linear bipolar cells. On the one hand, Burkhardt and colleagues used very high contrast changes which probably explain the saturation $(+10000 \%$ (Burkhardt and Fahey, 1998) and $+1000 \%$ (Fahey and Burkhardt, 2003) compared to the $\sim \pm$ 30\% from the white noise analysis (Baccus and Meister, 2002)). On the other hand, they used an asymmetric increase $(+10000 \%$ or $1000 \%)$ and decrease (-100\%) of contrast, which further might explain the small hyperpolarization compared to the depolarization. The stimulus design from Burkhardt and colleagues might be criticized and considered as "unnatural"; however, they observed nonlinear effects in the bipolar cells already at small contrast steps of $\pm 20 \%$.

To investigate the output function in bipolar cells, we used both white noise stimuli and contrast steps. The white-noise stimuli were similar to the previously mentioned studies $( \pm 30 \%$ contrast) (Baccus and Meister, 2002; Rieke, 2001). For the contrast steps, we chose symmetric steps with an increase and decrease of $100 \%$ contrast. We observed nonlinear stimulus-response transformation with both white noise as well as contrast steps. Further, we also found that cells showing a nonlinear transformation in the white noise stimulus also showed nonlinear responses for the contrast steps. Thus, overall we think that the nonlinearities can be observed with both types of stimuli. 
Why do we see nonlinearities with white noise stimulation while previous studies mainly reported linear transformation? We think the main reason for the difference is that previous studies only portrait few examples of bipolar cells and never systematically quantified and studied the stimulus-response transformation over a larger population. For example, when looking at the three examples in Baccus and Meister (2002) one can observe unequal amount of hyper- and depolarization in at least one cell.

\section{Spatial nonlinearity}

How bipolar cells integrate their presynaptic inputs was largely ignored and broadly assumed to be linear, due to the common view that photoreceptors are linear. Further, several studies supported the idea of linear spatial integration in bipolar cells indirectly. For example, Baccus et al. (2008) predicted the responses of bipolar cells to jittering gratings with high accuracy of $\sim 70 \%$ from the linear spatiotemporal filter. They concluded that if the bipolar cell would show a nonlinear spatial integration, the linear spatiotemporal filter could not describe the findings accurately. Yet, they only studied four bipolar cells and the grating width was $184 \mu \mathrm{m}$, which is much larger than the receptive field of bipolar cells. Thus, the position of half black and half white inside the receptive field might be rare, which increases the chance of a good prediction similar to a full-field stimulation. Further, Bolinger and Gollisch (2012) investigated the spatial scale of the integration in retinal ganglion cells. They found that squares with diameters below $50 \mu \mathrm{m}$, which is roughly below the receptive field size of bipolar cells, lead to linear spatial integration in ganglion cells. Thus, the results were interpreted as a sign that bipolar cells integrate linearly, because if they do a nonlinear integration, a nonlinear response in retinal ganglion cells to diameters below $50 \mu \mathrm{m}$ could be expected. A reason for the findings in Bolinger and Gollisch (2012) might be that they only focused on retinal ganglion cells with large, homogenous spike shapes. Thus, other retinal ganglion cells could have shown nonlinear responses to small squares below $50 \mu \mathrm{m}$. Further, for us $\sim 50 \%$ of the bipolar cells had receptive field sizes between $100-150 \mu \mathrm{m}$, thus square sizes above $50 \mu \mathrm{m}$ could already subdivide the receptive fields of bipolar cells and lead to nonlinear integration. Similarly, Ölveczky et al. (2003) concluded that bipolar cells integrate linearly because the minimum width of the grating for a retinal ganglion cell to get activated is $\sim 20-40 \mu \mathrm{m}$. Yet, a size of $20-40 \mu \mathrm{m}$ is for $90 \%$ of our recorded bipolar cells clearly below their receptive field size and already subdivides the receptive field into two halves. Thus, from recordings of retinal ganglion cells it might not be straightforward to infer whether bipolar cells integrate linearly or nonlinearly.

To our knowledge, only one study tested for explicit nulling of the bipolar cell response to a grating stimulus. Borghuis et al. (2013) measured the glutamate release from bipolar cell 
terminals to a reversing grating of $650 \mu \mathrm{m}$ diameter that was centered onto a simultaneous recorded retinal ganglion cell. They found that bipolar cells did not respond with frequency doubling to the grating and at certain spatial phase, they stayed silent. That bipolar cells would not show frequency doubling over all spatial phases can be easily explain by the width of the grating that is much larger than the receptive field of bipolar cells. Thus, bipolar cells would either be covered by the bright or dark bar and therefore not show a frequency doubling. Yet, that they found a nulling of the response to some phases is a strong sign for a linear integration. It seems accurate to assume that at this phase, both dark and white bars were present inside the receptive field and therefore bipolar cells did not respond. The results could be explain by a small amount of recorded cells. Further, they did not show population analysis of their findings and for the single examples, they only showed the F1 amplitude and not the F2 amplitude. The F2 amplitude is the important frequency to study whether frequency doubling i.e. nonlinear integration occurred. Other critics could be that they only used a UV light (peak $395 \mathrm{~nm}$ ) source that equally stimulated M and S cones ( $\sim 20 \%$ absorption for both). If a UV and green light source would be used with peak wavelength closer to the s-cones $(306 \mathrm{~nm}$ peak sensitivity) and m-cones (508 nm peak sensitivity), the reported nonlinear release at the photoreceptors might be triggered more effectively (Baden et al., 2013c).

\subsection{Circuit mechanisms for nonlinearities in bipolar cell}

Here, we discuss potential biological mechanism that might underlie the nonlinearities observed in bipolar cells. We first investigate the stimulus-response transformation, then the spatial integration. Yet, as we will see, the mechanism for the two types of nonlinearities are strongly connected to each other. Note, even though our focus is on nonlinearities, we briefly discuss potential mechanism for linear signal transmission too.

\section{Nonlinear stimulus-response transformation}

Diverse studies showed approximately linear stimulus-response transformations when recording from bipolar cell somas (Baccus and Meister, 2002; Rieke, 2001; Sakai and Naka, 1987a, b; Sakai et al., 1995; Toyoda, 1974). ${ }^{5}$ Yet, measurements of excitatory currents in ganglion cells indicated (Liang and Freed, 2010; Molnar et al., 2009; Zaghloul et al., 2003) and direct

\footnotetext{
${ }^{5}$ When looking closer at the reported linear transformations, saturations can be observed for preferred stimuli. Thus, the bipolar cells had at certain point stronger hyperpolarization than depolarization. Yet, rectification or in other words, higher depolarization than hyperpolarization was not reported.
} 
glutamate imaging from the axon terminals of bipolar cells showed, nonlinear neurotransmitter release (Borghuis et al., 2013; Franke et al., 2017). Thus, it was proposed that the nonlinear effect of bipolar on retinal ganglion cells occur at bipolar cells' axon terminals after the soma (Baccus et al., 2008). The idea is that at bipolar cell somas equal amounts of hyper- and depolarization are present, yet at the synapse only the depolarization leads to neurotransmitter release, while the hyperpolarization has no effect and does not reduce the neurotransmitter release (Roska and Meister, 2014). Such a rectification at the synapse needs a low baseline transmitter release, which can arise from resting potentials that are below the activation of voltage-dependent calcium channels (Matsui et al., 1998; Palmer, 2010). Moreover, spike-like events were observed in bipolar cell terminals under calcium imaging that were more transient than those from voltage depolarization, which would further add to nonlinear transmitter release (Baden et al., 2013a; Baden et al., 2011; Dreosti et al., 2011), for a review see also (Baden et al., 2013b). However, in our study, as well as other studies, nonlinear signal transmission was already observed when recording signals from the soma of bipolar cells (Burkhardt and Fahey, 1998; Euler and Masland, 2000; Fahey and Burkhardt, 2003). How to explain such nonlinear transformations at the soma of bipolar cells? Roughly, four mechanism can be summarized from the literature. 1. Nonlinear mechanism at photoreceptor-horizontal cell neurotransmitter release 2. Nonlinear mechanism at the bipolar cell dendrites. 3. Nonlinear mechanism at the bipolar cell soma. 4. Nonlinear mechanism at the bipolar cell axonal terminals. See Figure 7.2 for a summary of the four different mechanism. We briefly explain each mechanism separately.

\section{Nonlinear transmitter release at photoreceptor-horizontal cells. The first mechanism proposes a} nonlinear release in photoreceptors. Commonly, photoreceptors are viewed as linear and several studies showed linear voltage responses in cone photoreceptors (Baccus and Meister, 2002; Baylor et al., 1974; Burkhardt and Fahey, 1998; Rieke, 2001). Yet, recent studies that imaged calcium-signals at the photoreceptor terminals found strong rectification in some subtypes of cones in the mouse (Baden et al., 2013c). Further, even spike-like events have been claimed in photoreceptors from amphibians to humans, for a review see (Baden et al., 2013b). Yet, the recording conditions were rather unnatural, for example spike-like events were observed in isolated cone photoreceptors (Maricq and Korenbrot, 1988) or after strong hyperpolarization in rods (Kawai et al., 2001). For rods, it is broadly accepted that they have a nonlinear stimulus-response transformation, which allows them to detect dim light (Dunn and Rieke, 2008; Field and Rieke, 2002; van Rossum and Smith, 1998). Commonly it is thought that rods are not active under daylight conditions (similar to the one used in our experiments), yet this view has been challenged (Tikidji-Hamburyan et al., 2017). Thus, the nonlinear signal 
release from rods could be a potential source of nonlinearities also under daylight conditions. Horizontal cells could be another source for nonlinearities in bipolar cells, either through direct or indirect influence over the photoreceptors. Yet, the importance of horizontal cells for the retina is not well understood. However, in some studies the stimulus-response transformation in horizontal cells showed a degree of nonlinearity (Baccus and Meister, 2002; Rieke, 2001).

2. Nonlinear mechanism at dendrites. It has been proposed that the nonlinear transformation originates at different receptor types in bipolar cell dendrites and that ionotropic glutamate receptors might be the main source for the nonlinear transformation (Demb et al., 2001a). The idea gains support by diverse studies showing that OFF bipolar cells (ionotropic receptors) exhibit more nonlinear glutamate release compared to ON bipolar cells (metabotropic receptors). The results were observed under direct measure of glutamate release at bipolar cell terminals (Borghuis et al., 2013; Franke et al., 2017) as well as when measuring excitatory currents in retina ganglion cells (Liang and Freed, 2010; Molnar et al., 2009; Zaghloul et al., 2003). Further, there is some indication that the nonlinear bipolar cells are more transient (which might come from AMPA receptors, see subchapter 2.1) and stratify in the center of the inner plexiform layer (Borghuis et al., 2013). Other potential, but less understood mechanism, could be voltage-dependent sodium (Zenisek et al., 2001), potassium (Klumpp et al., 1995; Yazulla and Studholme, 1998; Yu et al., 2009) or calcium channels (Nawy, 2000; Rieke, 2001; Shiells and Falk, 1999) that were localized at the bipolar cell dendrites.

3. Nonlinear mechanism at soma. Voltage-dependent sodium (Zenisek et al., 2001), potassium (Yazulla and Studholme, 1998) and calcium (Satoh et al., 1998) channels were observed in the soma of bipolar cells. Thus, the voltage-gated channels at the soma may play an additional role in shaping the bipolar cell response, for example by enhancing small depolarized potentials (Zenisek et al., 2001). Further, Mao et al. (1998) showed a degree of nonlinear dependency between the current injected and voltage measured at the soma of bipolar cells.

4. Nonlinear mechanism at axon terminals. Bipolar cells are short and compact neurons and therefore it has been proposed that a recording from the bipolar cell soma, does not simply show the response to the input signals at the dendrites but also the effects of amacrine cells (Eggers and Lukasiewicz, 2010; Euler and Masland, 2000; Masland, 2012a). Further, it was also speculated that the diverse voltage-dependent calcium (Satoh et al., 1998) and potassium (Klumpp et al., 1995) channels could influence the signals at the soma of bipolar cells (Euler and Masland, 2000). Thus, it might be possible that the nonlinear transformation measured in the soma comes from the effects in the axon terminals. Yet, diverse studies that pharmacologically 
blocked different kinds of amacrine cells showed that amacrine cells are not involved in shaping the output nonlinearity in bipolar cells (Demb et al., 1999; Demb et al., 2001a; Euler and Masland, 2000). However, in a recent study the blocking of glycinergic amacrine cells reduced but did not abolish the rectification in OFF bipolar cells (Franke et al., 2017).

Just from our observed nonlinear output functions, it is difficult to exclude or favor one of the proposed mechanism. Yet still, the findings provide enough material for speculations. For example, a potential candidate could be mechanism 2: the receptors of dendrites. We found a difference among OFF bipolar cell types, the more transient the cells were in the center, the more nonlinear their response. As we have reviewed in subchapter 2.1, transient OFF bipolar cells are shown to exhibit different receptor-types at their dendrites compared to sustained OFF bipolar cells (AMPA vs. kainate). Furthermore, we found that ON bipolar cells showed more linear output functions with saturation, while OFF bipolar cells showed linear and nonlinear responses without saturation. These findings are in line with mechanism 2, because $\mathrm{ON}$ and OFF bipolar cells exhibit different receptor types (metabotropic vs. ionotropic).

Mechanism 3, the nonlinear transformation at the soma might be another candidate for nonlinear output functions observed in bipolar cells. The reported voltage-gated channels at bipolar cell somas might lead to a nonlinear transformation of the signal, after the integration. For example, it could be that if the membrane potential of the bipolar cell reaches a certain level, voltage-gated channels at the soma open and amplify the signal.

Mechanism 4, the amacrine cell influence on the axon terminal that back-propagates to the soma might be less involved. Usually the influence of amacrine cells is considered as more pronounced under stimulation that exceed the receptive field center size. For example, a recent study documented that bipolar cells exhibit very different and more diverse response profiles under full-field compared to center stimulation (Franke et al., 2017). Further, pharmacological blocking of amacrine cells had a strong effect on responses to full-field light stimulation but little effect under center stimulation. We found rectification both under full-field and center stimulation, thus our results were quite stable and did not change with the activation of amacrine cells. Yet, in the same study from Franke et al. (2017), it was shown that narrow-field glycinergic amacrine cells can play a role also under local stimulation by changing the ratio between hyperand depolarization. However, the hyper- and depolarization ratio was still quite nonlinear even under the blocking of narrow-field amacrine cells. At this point, only pharmacologically blocking of different amacrine cell types could reveal their influence on the nonlinear stimulus-response transformation. 
For mechanism 1, the photoreceptor-horizontal cell release side, we found that the recorded photoreceptors in our experiments $(n=10)$ showed very linear stimulus-response transformations. Thus, mechanism 1 might be less plausible. However, nonlinear release at the photoreceptor side could still be possible. On the one hand, we only recorded few photoreceptors and on the other hand, the neurotransmitter release might differ from the recorded voltage signal. Thus, further recordings from different cone photoreceptors as well as simultaneous recordings from one photoreceptor and one bipolar cell could shine light on the role of photoreceptors for nonlinear signal transmission. For example, there are indication, that the photoreceptors are linear, yet from the transmission to the bipolar cells the signal becomes nonlinear (Burkhardt and Fahey, 1998).

Another way to disentangle the four mechanism is by looking at the prediction they make about how the bipolar cells integrate signals in space. The degree of nonlinearity in the spatial integration is strongly correlated with the nonlinearity observed in the stimulus-response transformation. Thus, it might be that the mechanisms for the two types of nonlinearities are similar. In a next step, we are going to inspect the mechanisms in the light of the spatial integration.

\section{Nonlinear spatial integration}

We observed bipolar cells that sum presynaptic inputs linearly and bipolar cells that sum the inputs nonlinearly. Further, we found that bipolar cells with transient responses in the center showed more nonlinear spatial integration response and bipolar cells with sustained responses in the center were more linear in their input integration.

Again, we think that our results favor mechanism 2, a nonlinear transformation at the bipolar cell dendrites. We favor mechanism 2 because we found that different bipolar cell types (e.g. sustained vs. transient) exhibited different nonlinear spatial integration properties. Furthermore, the temporal properties of the center and not the surround were related to the spatial nonlinear integration properties. As we have seen in subchapter 2.1, the different receptors and channels in bipolar cells form different response properties like sustained and transient (kainate vs. AMPA receptors). Further, the center of a bipolar cell is mainly driven by direct excitatory inputs to the dendrites. Thus, it might be that the different temporal dynamics of the receptors at the bipolar cell dendrites lead to a nonlinear transformation and to a subsequent nonlinear integration.

For mechanism 1, it has been speculated that if photoreceptors would respond nonlinearly, then all bipolar cells and ganglion cells should respond nonlinearly (Demb et al., 2001b). We clearly see linear and nonlinear bipolar cells, which might indicate that a mechanism 
at the bipolar cell dendrites separates the photoreceptor signal in different ways. Yet, a recent study showed that not all photoreceptors respond to light in the same way and that some types can show rectification (Baden et al., 2013c). Thus, it could be that the observed nonlinear bipolar cells make specific synaptic connections to photoreceptors with nonlinear response properties. Further, horizontal cells might influence the signal of bipolar or photoreceptors nonlinearly for different bipolar cell types. To disentangle the influence of horizontal cells, pharmacological experiments would be needed (Purgert and Lukasiewicz, 2015; Rieke, 2001), for a review see also (Thoreson and Mangel, 2012). However blocking horizontal cells especially through inhibition of GABA receptors, like for example in Rieke (2001), also effects amacrine cells that exhibit similar receptor types. Another, maybe "cleaner" way to study the signal transmission in the outer retina would be to directly record the signals from horizontal cells or even combined recordings from horizontal and bipolar cells or photoreceptors.

Both mechanism 3 and 4 would predict a linear spatial integration, because the nonlinear transformation occurs after the summation of the input signals by the bipolar cells. Therefore, they seem to be less likely involved in the spatial integration. If the nonlinearity observed in the stimulus-response transformation were only due to voltage-gated channels at the soma or axon, we would not observe a nonlinear spatial integration and no correlation between the two types of nonlinearities. Thus, our results indicate that most of the observed nonlinear stimulus-response transformation occurs before the summation of the signals by the bipolar cells. Similar has been proposed for retina ganglion cells, where the excitatory input closely matches the output nonlinearity after spike generation (Schwartz and Rieke, 2011). However, it might still be that channels at the soma or axon, as well as amacrine cells, influence the signal recorded at the soma and add additional nonlinear effects measured in the output nonlinearity, yet not influencing the spatial integration. Pharmacological blocking of amacrine cells might help to study the influence of different amacrine cell types (Eggers and Lukasiewicz, 2011; Franke et al., 2017; Kuhn and Gollisch, 2016; Purgert and Lukasiewicz, 2015).

Note, the common belief is that nonlinear spatial integration can only occur through a nonlinear transformation before the summation. Yet, as has been recently shown, it is possible to get a nonlinear spatial integration that occur from a linear transformation before the summation (Borghuis et al., 2013). Borghuis et al. (2013) investigated how from a linear release in ON bipolar cells, retinal ganglion cells can show nonlinear spatial integration. They found a temporal asymmetry in the increase of glutamate release form the baseline compared to the decrease of glutamate release. The idea is shown in Figure 7.2, as an additional mechanism 5. Thus, it might be possible, that the photoreceptor release is linear and also the dendrite of bipolar cells are linear, yet the bipolar cell responds nonlinearly to the inputs, because there is a temporal 
difference between the increase and decrease of neurotransmitter release. The temporal asymmetry might originate from the photoreceptor or dendrite side of the bipolar cells.

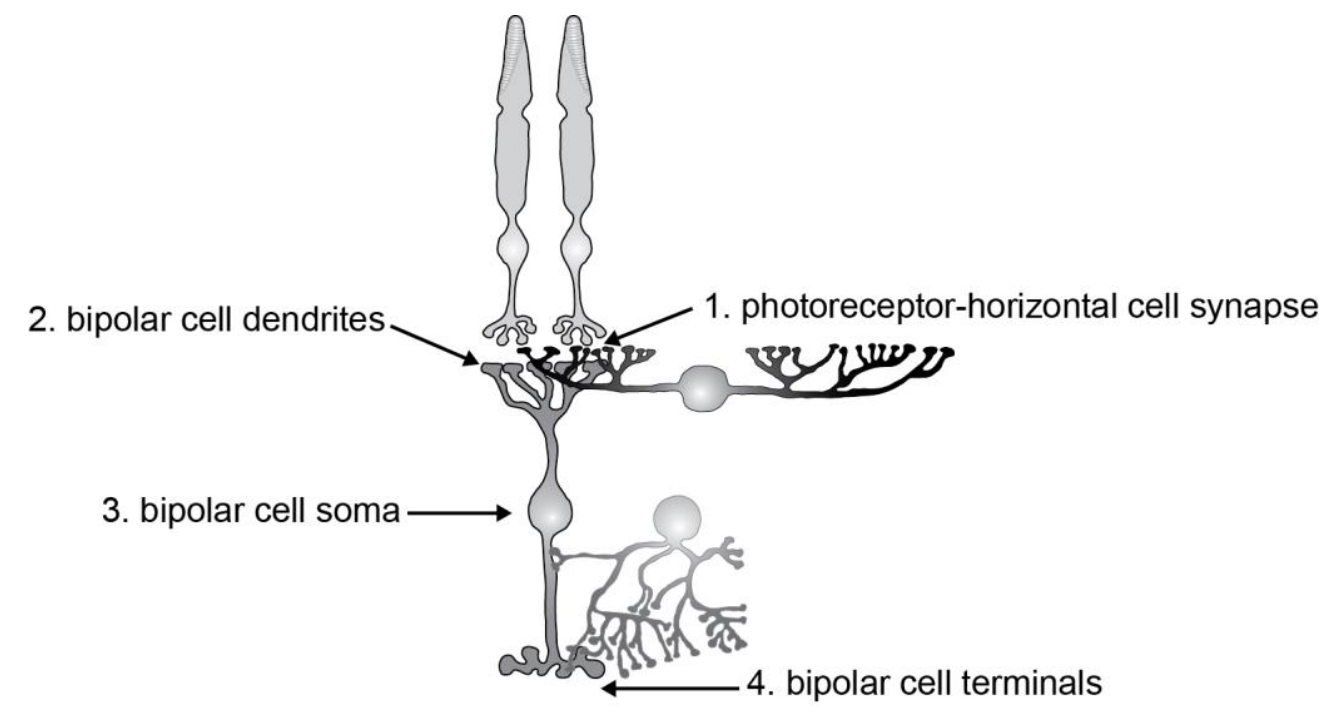

5. temporal delay

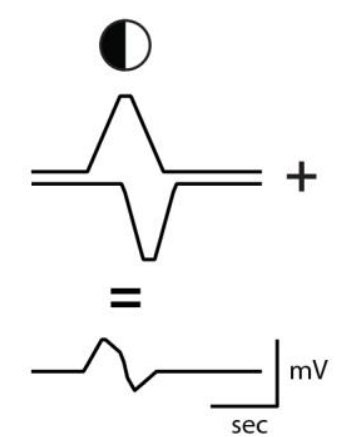

Figure 7.2. Five possible mechanism for the nonlinearities observed in bipolar cells. Mechanism 1 describes a nonlinear signal transformation at the photoreceptor-horizontal cell side. Mechanism 2 a nonlinear transformation at the bipolar cell dendrites. Mechanism 3 a nonlinear signal transformation at the soma, after the integration and mechanism 4 a nonlinear transformation at the bipolar cell terminals that back propagates to the soma. Mechanism 1-2 are candidates for nonlinear spatial integration. For the nonlinear output transformation, mechanism 1-4 could be involved. Mechanism 5 shows the idea of a temporal delay in the input signals proposed by Borghuis et al. (2013). The mechanism is an additional explanation for nonlinear spatial integration.

\section{Linear bipolar cell responses}

Around 30\% of our recorded bipolar cells showed linear stimulus-response transformation and spatial integration. What causes bipolar cells to be linear? In principal, all presented mechanism could be candidates for a linearization of the bipolar cell response. We favor mechanism 2, the dendrites. We have found more linear responses in ON bipolar cells and sustained OFF bipolar cells, both of them exhibit different receptor types (metabotropic and 
ionotropic kainate receptors, compared to the ionotropic AMPA receptors in the nonlinear cells). Thus, the receptor types at the dendrite might play a key role (Demb et al., 2001a). For the third and fourth mechanism, it might be that the linear cells exhibit different voltage-gated channels at their soma or axon. For example, ON bipolar cells show linear glutamate release, which needs a high baseline level so that a hyperpolarization can reduce the glutamate release by roughly the same amount that a depolarization increases the glutamate release (Borghuis et al., 2013; Zaghloul et al., 2003). Thus, the resting potential of a linear cell should not be below the activation of voltage-dependent calcium channels or the voltage-dependent calcium channels should have a different threshold. Generally, it is reported that ON bipolar cells show a higher resting potential than OFF bipolar cells. For mechanism 1, the release site of photoreceptor-horizontal cells it could be that the linear bipolar cells only connect to linear photoreceptors. Further, one might speculate that horizontal cells could linearize the photoreceptor release similar to amacrine cells that linearize the bipolar cell release. Furthermore, it could be possible that we record the linearization from amacrine cells at the bipolar cell soma. A linearization of the bipolar cell release by an amacrine cell is termed crossover inhibition (Molnar et al., 2009; Werblin, 2010). The idea is that a rectified OFF bipolar cell that shows little hyperpolarization to light is influenced at the terminal by an amacrine cell that itself is activated from an ON bipolar cell. The amacrine cell would provide the inhibitory effect, similar to the missing hyperpolarization. Yet, in a recent study it was shown that the cross-over inhibition from the ON bipolar cells to the OFF bipolar cells via a glycinergic amacrine cells, does not linearize but rectifies the release in the bipolar cells (Franke et al., 2017). Thus, the crossover inhibition as a mechanism for linearization might be less relevant for bipolar cells directly. Yet, pharmacological experiments might clarify whether there is a linearization and whether amacrine cells are involved.

\subsection{Consequences of nonlinearities in bipolar cells for models in retinal ganglion cells}

In the previous sections, we investigated the roles of nonlinearities for bipolar cell encoding. Here, we discuss the implications of nonlinearities in bipolar cells for modelling retinal ganglion cell responses. 
Impact of the output nonlinearities in bipolar cells on models in retinal ganglion cells

The classical linear-nonlinear model for ganglion cells (Fig.7.3A) assumes a linear signal transmission from the photoreceptors to bipolar to retinal ganglion cells (first stage). Then, the signals are summed by the ganglion cells and transformed through a cell internal nonlinear process (spike threshold and saturation) to generate a response (second stage). The model is quite successful at predicting retinal ganglion cell responses to uniform contrast stimuli. Yet, the model fails for artificial and natural stimuli (Heitman et al., 2016; Liu et al., 2017; Turner and Rieke, 2016). It is hypothesized that the model fails because of nonlinear spatial integration in retinal ganglion cells (Gollisch, 2013; Schwartz and Rieke, 2011). Nonlinear spatial integration is attributed to rectifying bipolar cells (Borghuis et al., 2013; Demb et al., 2001a), which contradict the assumed linear signal transmission from bipolar to retinal ganglion cells proposed by the linear-nonlinear model.

In our study, we show that some bipolar cells indeed do a nonlinear transformation of the input signals. We found nonlinear stimulus-response relationship in diverse shapes, for example threshold-linear, threshold-quadratic, partially rectifying linear, partially rectifying quadratic. Thus, our findings agree with the general notion that the linear-nonlinear model has to include an additional nonlinear transformation at the bipolar cells before the summation by retinal ganglion cells. The LN-model for retinal ganglion cells should therefore be extended to a LN-LN-model (see Fig.7.3B). The input signals are first nonlinearly transformed by the bipolar cells before summed by the ganglion cell. Yet, as we have already mentioned, implementing an additional nonlinear transformation comes with challenges.

Classically, the receptive field of retinal ganglion cells is subdivided into small subunits that first filter and integrate the light stimulus and then transform it nonlinearly. Such models are also termed subunit models and face certain challenges: How to choose the subunits location and size inside the ganglion cell receptive field? Does the subunits correspond to bipolar cells or to a mixture between bipolar and amacrine cell signals? In a recent study from our group, we introduced a new method termed spike-triggered non-negative matrix factorization (STNMF) that identifies the location and size of individual subunits (Liu et al., 2017). By combined recordings from retinal ganglion cells and bipolar cells, we found that the retrieved subunits correspond to individual receptive fields of presynaptic bipolar cells. We also found that the prediction of retinal ganglion cell responses improves with an additional nonlinear transformation at those subunits. Thus, our method can recover properties of unobserved upstream neurons without the need of anatomical reconstruction as done in Schwartz et al. (2012).

However, certain challenges are left. For example, currently the bipolar cell nonlinear transformation is often approximated as threshold-linear in the subunit model. Further, for all 
bipolar cell subunits the same nonlinear transformation is chosen. Yet, as we have seen, different bipolar cells show different transformations, ranging from fully linear to threshold-quadratic transformations. A next stage would be to improve the subunit model for each retinal ganglion cell by using the "real" nonlinear transformation of the connected bipolar cells. The introduced method of combined recordings in our goal 4 (see method subchapter 3.3) provide a technical approach to investigate the connections between bipolar and retinal ganglion cells. For example, one could measure the stimulus-response transformation from multiple bipolar cells in one region and assess their connections to the simultaneous recorded retinal ganglion cells by current injection. Then, one calculates the subunits of the retinal ganglion cells with the STNMF method and for the prediction one uses the "real" measured nonlinear transformation of the corresponding bipolar cells. Yet, this approach depends strongly on the experimental success of recording most connected bipolar cells in a small region.

Another approach could be to infer from the type of recorded retinal ganglion cell the type of connected bipolar cells. Herefor, one first has to quantify the connections between bipolar cell types and retinal ganglion cells. The introduced combined recording technique provides a possible approach. For example, if all sustained retinal ganglion cells are connected with threshold-linear bipolar cells, the subunit model could be generalized to use only thresholdlinear subunits for sustained ganglion cells. On the other hand, if all transient retinal ganglion cells are connected to threshold-quadratic bipolar cells, the subunit model could be generalized to include threshold-quadratic transformations only for those cell types. In all our experiments, we recorded simultaneously the bipolar cell signals and ganglion cell responses. Thus, with our recorded data it would be interesting to study the connections between the different observed bipolar cell types and the known ganglion cell types.

A critical aspect when studying these questions is that we measure signals from the soma. However, the signals that influence the retinal ganglion cells come from the bipolar cell terminals. Here, it was proposed that the signal transmission at the terminals can be nonlinear even though the soma is linear (Baccus et al., 2008). Thus, it might well be that the linear or nonlinear transformation measured at the soma does not reflect the influence of the bipolar cell onto the retinal ganglion cell. Further, the influence of amacrine cells might linearize (Molnar et al., 2009; Werblin, 2010) or even enhance nonlinear signal transmission (Franke et al., 2017). It would be interesting to study how the signals at the soma differ from the vesicle release at the bipolar cell terminals. To study this question, simultaneous recordings at the soma and glutamate or calcium imaging at the terminals would be valuable. 
Impact of spatial nonlinearities in bipolar cells on models in retinal ganglion cells

Until now, we have looked at the role of nonlinear bipolar cells in respect to their output function. Yet, as we have further seen, bipolar cells themselves integrate information nonlinearly. This calls for an even further change of the subunits model to an LN-LN-LN model to capture retinal ganglion cell responses. The idea is pictured in Figure 7.3C. The light signal is first filtered by the photoreceptors and then nonlinearly transformed, either at the photoreceptor-horizontal cell synapse or at the bipolar cell dendrites (1.LN). In a next step, the signals are summed by the bipolar cells and again nonlinearly transformed at the soma and terminals of bipolar cells (2.LN). In the last step, retinal ganglion cells sum the signals and pass them through their own nonlinear transformation to generate spikes (3.LN).

A

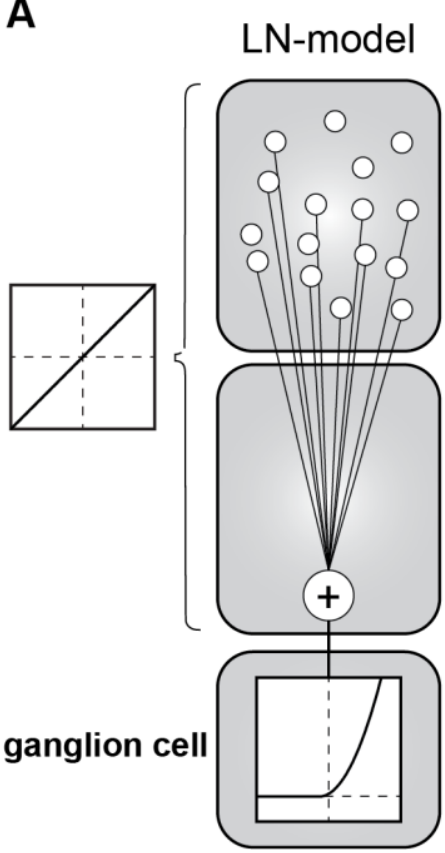

B

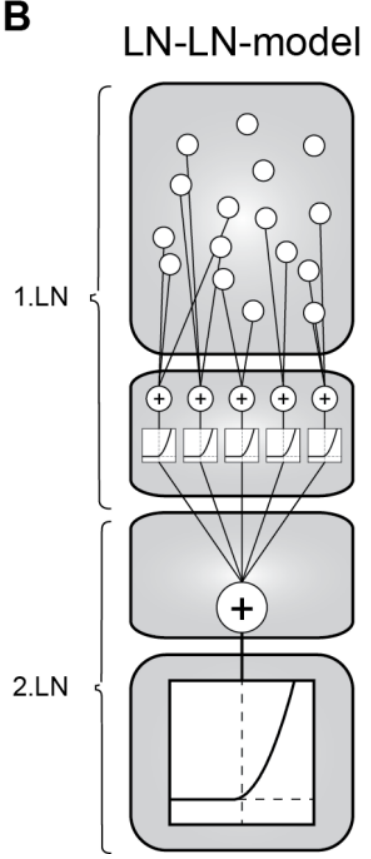

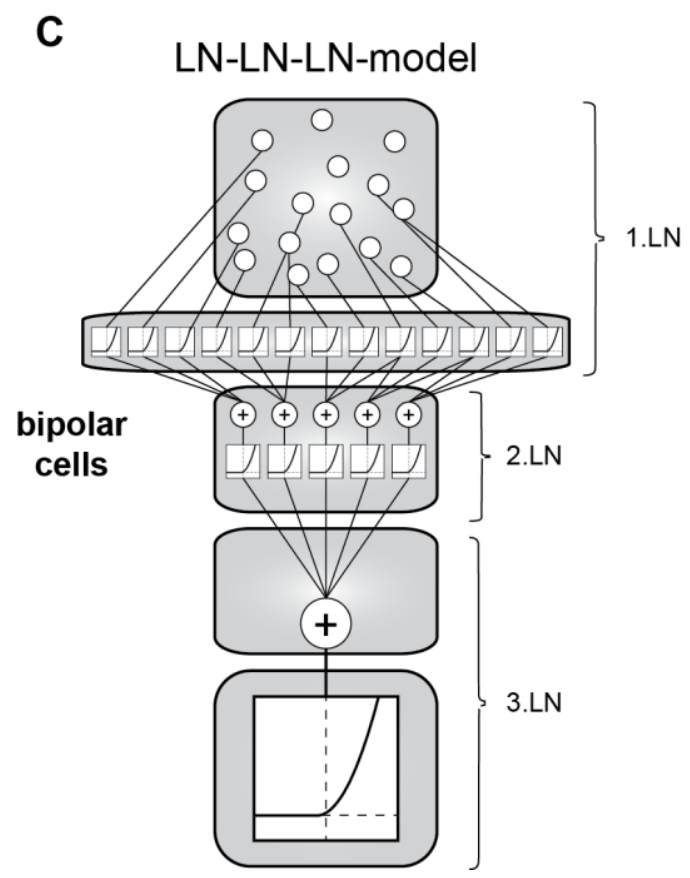

C

Figure 7.3. Implication of nonlinearities in bipolar cells for models in retinal ganglion cells. A. Simple linear-nonlinear model (LN-model) for retinal ganglion cells (RGC). The signals are linearly filtered, summed by the ganglion cell and then nonlinearly transformed by the output function of the ganglion cell. B. Proposed extension of the $\mathrm{LN}$-model with an additional nonlinear transformation at the level of the bipolar cells before the summation (LN-LN-model). C. Proposes extension to an LN-LN-LN model, the signals are first nonlinearly transformed before the summation by the bipolar cells. Then, the signals are passed through the nonlinear transformation of the bipolar cells and summed by the ganglion cell. At the last stage, the signals are passed through the ganglion cell's own nonlinear function. The subfigure A \& B was inspired by Freeman et al. (2015). 
One might wonder, whether the nonlinear integration by bipolar cells is at all relevant for retinal ganglion cells. In the next section, we are going to discuss the general function of nonlinearities in bipolar cells.

\subsection{Possible functions of bipolar cells' nonlinearities and linearities}

Until now, we discuss the role of nonlinearities in bipolar cells for encoding in the context of the linear-nonlinear model. Yet, what function is served by the nonlinearities for vision? Here, we discuss possible functional roles of the bipolar cells' nonlinearities and linearities for the visual feature extraction by retinal ganglion cells.

\section{What function is served by the nonlinear stimulus-response transformation in bipolar cells?}

It has been proposed, that the nonlinear transformation at the output of bipolar cells lead to the so-called Y-retinal ganglion cells. A possible function of Y-retinal ganglion cells is to extract patterns with high spatial structure or small high-contrast objects inside their receptive fields (Demb et al., 2001a; Gollisch, 2013). For example, through nonlinear transformation a white object that is the size of the bipolar cell receptive field can be detected on a black background by ganglion cells. If the signals are not nonlinearly transformed at the level of bipolar cells, they would cancel with the opposite background signals and the retinal ganglion cells would stay silent. Yet, whether the Y-type retinal ganglion cells are only connected to bipolar cells with nonlinear output functions has not been studied. Here, the combined recordings, introduced as goal 4 in our method section, would provide an approach to study the connections between the nonlinear bipolar and ganglion cells. Surprisingly, it has recently been shown, that even linear bipolar cells can create Y-type retinal ganglion cell responses through temporal delays between the increase and decrease of neurotransmitter release (Borghuis et al., 2013). Thus, if linear bipolar cells can also create higher sensitivity to spatial patterns, what is then the role of nonlinear bipolar cells? Diverse other roles are proposed, for example the extraction of small objects that move differently from the background (Baccus et al., 2008), the extraction of expanding objects (Munch et al., 2009) or the extraction of repeated images (Krishnamoorthy et al., 2017). Yet, it has never been studied whether those retinal ganglion cells are connected only to nonlinear bipolar cells or whether also linear bipolar cells could generate such feature extraction. Thus, it 
would be interesting to systematically study with the combined recordings whether the different ganglion cell types are connected to linear or nonlinear bipolar cells.

A further way to assess the role of nonlinear signal transmission between bipolar and ganglion cells would be to manipulate the bipolar signal directly. For example through current injection one could try to linearize the recorded bipolar cell and study whether the retinal ganglion cells change their response properties. One could hypothesize that a white object of the size of the recorded bipolar cell would cancel with the black background if the signal transmission from bipolar to ganglion cells would be linearized through current injection. Only through such direct manipulation, one can finally assess the functional roles of nonlinearities. However, as we have seen, the signals that influence the retinal ganglion cells come from the bipolar cell terminals. Thus, it might well be that a linearization at the soma through current injection would still lead to a nonlinear release. It would be interesting to study how the signal at the soma differ from the vesicle release at the bipolar cell terminals and if through current injection the release at the terminals could be linearized. Further, amacrine cells might linearize (Molnar et al., 2009; Werblin, 2010) or even enhance nonlinear signal transmission (Franke et al., 2017). Thus, it could be that the mentioned retinal ganglion cells are connected to linear bipolar cells and the amacrine cell is creating the nonlinear transformation at the synapse.

We have observed different shapes of nonlinear transformations at the bipolar cells e.g. threshold-linear, threshold-quadratic or partially rectifying transformations. One could imagine that the different transformations have different effects on feature extraction in retinal ganglion cells. For example, a threshold-quadratic transformation might also lead to a sensitivity of lowcontrast objects, while a partially rectifying transformation needs high-contrast objects to avoid cancellation of the input signals. Generally, it would be interesting to study, whether the different types of ganglion cells are connected to the different observed shapes of nonlinear transformations in bipolar cells. However, again it might be that an observed partial rectification at the soma becomes a full rectification at the terminals.

\section{What function is served by the nonlinear spatial integration in bipolar cells?}

Similar to retinal ganglion cells, one could speculate that the nonlinear transformation before the summation allows bipolar cells to be sensitive to patterns with high spatial structure or small high-contrast objects inside their receptive field. For example, the nonlinear transformation of the signals would allow bipolar cells to detect a black object that is half the size of the receptive field from a white background (see for example Figure 7.4, where a nonlinear bipolar cell would detect the head of the penguin in the snow). Yet, what does such a spatial nonlinear integration by bipolar cells mean for retinal ganglion cells? 
A nonlinear spatial integration in bipolar cells would increase the spatial sensitivity of retinal ganglion cells to patterns that are even smaller than the bipolar cell receptive fields. Currently, the spatial sensitivity of retinal ganglion cells is interpreted to be roughly at the size of bipolar cell receptive fields (Jacoby and Schwartz, 2017; Krieger et al., 2017; Mani and Schwartz, 2017; Schwartz et al., 2012). Yet, there is growing evidence that the spatial sensitivity of ganglion cells can be even below the receptive field size of bipolar cells. For example, Freeman et al. (2015) stimulated single cone photoreceptors individually and recorded the responses of ganglion cells. They found that when stimulating two cones that projected onto the same bipolar cell simultaneously with a dark and a light spot, the ganglion cell did not cancel its response. The finding is a strong sign that retinal ganglion cells are sensitive to spatial structure at a photoreceptor level. Freeman et al. (2015) also discussed that nonlinearities in cone signals might be important to incorporate into the LN-model.

Diverse other studies showed sensitivity of retinal ganglion cells to spatial structures below the size of bipolar cell receptive fields (Jacoby and Schwartz, 2017; Krieger et al., 2017; Mani and Schwartz, 2017; Schwartz et al., 2012). However, they all interpreted their findings as an indication that the retinal ganglion cell spatial resolution is limited by the bipolar cell receptive field and not by structures that are below the bipolar cell receptive field size. For example, Krieger et al. (2017) and Jacoby and Schwartz (2017) reported responses in retinal ganglion from spatial structures of $\sim 25 \mu \mathrm{m}$. Schwartz et al. (2012) and Mani and Schwartz (2017) reported responses in retinal ganglion cells to spatial structures of even $\sim 10 \mu \mathrm{m}$. Their interpretation that the responses of retinal ganglion cells to the small structure is driven by the resolution of the receptive field size of bipolar cells can be criticized from two points. First, in the mouse retina, the bipolar cell receptive field sizes are reported between $40-80 \mu \mathrm{m}$ with white-noise (Franke et al., 2017), around 66-80 $\mu \mathrm{m}$ with spots (Borghuis et al., 2013) and with bars around $44 \mu \mathrm{m}$ in whole mount (Schwartz et al., 2012) and 43-67 $\mu \mathrm{m}$ in slice (Berntson and Taylor, 2000). Thus, the 10 or $25 \mu \mathrm{m}$ probably already subdivided the bipolar cell receptive field into two or more halves and the responses of the ganglion cells could as well be triggered by a nonlinear integration in the bipolar cells. Here, the authors of the mentioned studies might counter argue that at least the 25 $\mu \mathrm{m}$ is roughly bigger than the smallest bipolar cell receptive field of $40 \mu \mathrm{m}$ and therefore activates the bipolar cells enough without the need of a nonlinear integration. As a second argument, the difference between the receptive field sizes of $\mathrm{ON}$ and OFF bipolar cells has to be considered. ON bipolar cells are reported to have larger receptive fields than OFF bipolar cells (Borghuis et al., 2013; Franke et al., 2017). For Krieger et al. (2017) two of the reported ganglion cell classes that responded to $25 \mu \mathrm{m}$ were $\mathrm{ON}$ retinal ganglion cells. Thus, the $25 \mu \mathrm{m}$ would clearly subdivide the receptive fields of the $\mathrm{ON}$ bipolar cells, which are reported at around $80 \mu \mathrm{m}$ with spots and 
50-80 $\mu \mathrm{m}$ with white-noise (Borghuis et al., 2013; Franke et al., 2017). The studies with $10 \mu \mathrm{m}$ spatial resolution in ganglion cells attributed the effect to the spatial resolution of bipolar cells, because the maximum response in the ganglion cell was reported between 20-30 $\mu \mathrm{m}$. Yet, they did not explain the responses to $10 \mu \mathrm{m}$ spatial scale, which is clearly below the bipolar cell spatial resolution. Our results open the possibility for a new interpretation of these findings. Mainly that the responses of retinal ganglion cells to spatial structures of $10 \mu \mathrm{m}$ originate from nonlinear spatial integration in bipolar cells. Is the proposed nonlinear integration in bipolar cells relevant for the brain and behavior?

In dim light conditions, retinal ganglion cells can report the signal of single photons absorbed by rods to the brain (Barlow et al., 1971). Further, humans can report single photons absorbed by rods (Hecht et al., 1941). The pathway is achieved by nonlinear signal transmission from rods to bipolar to ganglion cells. If bipolar cells would sum the signals of rods linearly, a single photon would easily be canceled by the surrounding activity. Our findings could point to a similar pathway during daylight. The mechanism would allow transmitting the activation of single or multiple close by photoreceptors to the brain during daylight. The nonlinear signal transmission from photoreceptors to bipolar cells could help that signals do not cancel out, but can be further processed. It could even provide an alternative mechanism to keep a higher spatial resolution without the need of a fovea. The mouse as well as the salamander do not possess a fovea. The fovea has a one-to-one connection between photoreceptors, bipolar and ganglion cells, and underlies high spatial resolution. In the mouse and salamander, multiple photoreceptors synapse onto bipolar cells and ganglion cells and therefore the spatial resolution decreases. Thus, the nonlinear transformation from photoreceptors to bipolar cells could provide a way to allow higher spatial resolution than expected from pure averaging of the signals (see example of nonlinear cell in Figure 7.4).

What is the spatial resolution of mouse vision measured with behavior experiments? The highest spatial resolution was reported between $\sim 25-30 \mu \mathrm{m}$ (Sinex et al., 1979; Umino et al., 2008). Thus, below the receptive field size of bipolar cells. Therefore, it might well be that the reported findings of nonlinear spatial integration in bipolar cells have concrete behavior effects. Also for humans, behavior experiments suggested a nonlinear transformation at cones for highcontrasts (He and Macleod, 1998).

\section{What function is served by the linear bipolar cells?}

Neurons that integrate their signals linearly are sometimes referred to as pixel-sensors (Roska and Meister, 2014) or pixel-encoder (Johnson et al., 2018). What does a pixel-sensor do? The idea is that the neuron simply senses the light intensity inside its receptive field, similar to a 
digital camera and reports the value to the brain. For example, for a linear bipolar or ganglion cell, a stimulus with spatial structure would simply be reported as the sum over the different location with variable light intensities, sometime also referred to as averaging (see example of a linear cell that reports the penguin's head as gray Figure 7.4). For bipolar cells, it is generally assumed that they report the summed light intensity over the connected photoreceptors linearly. In our study, we showed that $30 \%$ of the bipolar cells perform such a linear summation. Thus, those bipolar cells might provide the basis for the reported idea of pixel sensors.

For the retinal ganglion cells, the X-cell was reported to linearly integrate light. The linear bipolar cells (both in the output and spatial nonlinearity) are speculated to be the source of the linear integration observed in the X- retinal ganglion cells. Yet, it has never been systematically studied whether linear bipolar cells are only connected to retinal ganglion cells that show a linear spatial integration. The introduced combined recording method provides an approach to study this question. Yet, as we have already mentioned, the connections between bipolar and ganglion cells might not be as straightforward as expected. For example, there is evidence that linear bipolar cells are connected to nonlinear ganglion cells (Borghuis et al., 2013). Further, it is speculated that bipolar cells with nonlinear release are connected to linear ganglion cells and their signals become linearized through amacrine cells (Werblin, 2010).

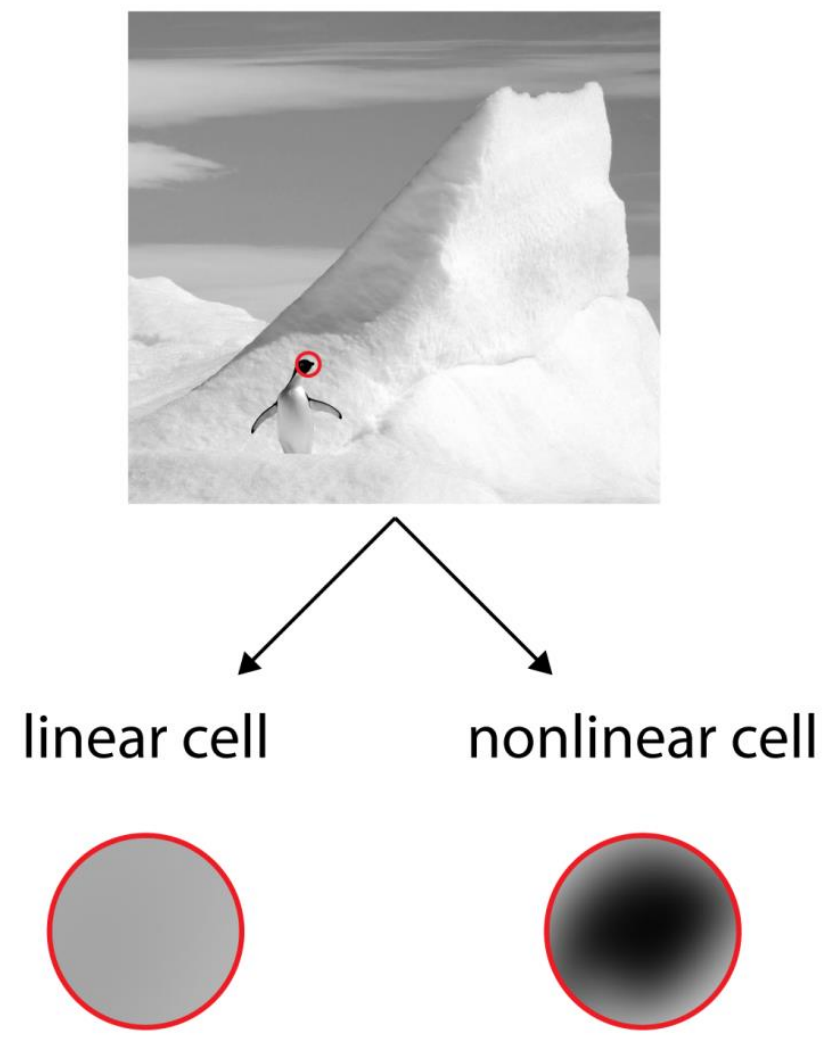


Figure 7.4. Possible function of linear and nonlinear spatial integration. Red circle shows the position of a bipolar cell's receptive field. The bipolar cell looks at the penguin's head where it is exposed to black and white contrast. The linear bipolar cell would report such a contrast combination as gray. The nonlinear bipolar cell would enhance the preferred contrast (black in the example) and report a dark image. Image was kindly provided by Julia Sondermann.

\subsection{Challenges in quantifying bipolar cell response types}

Here, we discuss some of the challenges encountered when quantifying the general response properties in bipolar cells. Characterizing general response properties in bipolar cells is often taken for granted. For example, knowing whether a bipolar cell is fast or slow or measuring the receptive field size, sounds like easy tasks. However, it turns out to be less straightforward and much more stimulus-dependent than conceived.

\section{Response polarity}

We found that for most bipolar cells the response polarity was stable over the different tested light stimuli (e.g. light steps with gray, light steps without gray, white-noise). Yet, curiously, we observed for some ON bipolar cells that the response can differ. For those cells, we found almost zero responses to a change of light from gray to white (light increment), however from dark to gray (again light increment) we observed strong responses. This was surprising because one usually expects that an $\mathrm{ON}$ cell would respond to a light increment to white. Experiments in the retina that try to assess the response polarity are either using light increment or decrement from a baseline background (usually gray) (Burkhardt and Fahey, 1998; Fahey and Burkhardt, 2003) or they use a switch between black and white, without a baseline background (Baden et al., 2013a; Borghuis et al., 2013; Franke et al., 2017). Our reported "anomaly" in ON cells could only be observed under a light step that starts from a baseline level. When probing the same cells with a light stimulus that switches between black and white directly, the trace of the cells looked like clear $\mathrm{ON}$ cells that responded to the white step of the stimulus. Thus, the results point out that simple black-white switches might not show the full scope of the response properties of bipolar cells and using light steps with intermediate gray might further subdivide the response polarity of bipolar cells. Further, some of these ON cells showed after an initial hyperpolarization to black a small positive peak that is again followed by further hyperpolarization. This patter was also present in the switch between black and white, however here, it sometimes led to the misinterpretation of an ON-OFF cell. Thus, it might be that the reported ON-OFF responses in 
bipolar cell terminals with the black-white switch (Baden et al., 2013a; Franke et al., 2017) are due to release of hyperpolarization and with an intermediate gray screen the ON-OFF response would disappear.

\section{Receptive field estimation}

We estimated the size of the receptive field center both with classical spots and with the binary white noise stimulus. Curiously, we found that most of the cells ( 95\%) have larger diameters when measured with spots compared to the binary white noise estimation. As we already mentioned, a similar discrepancy between the diameters is found in the literature both for salamander (compare for example Fahey and Burkhardt (2003) for spots and Ölveczky et al. (2003) for white noise) and mouse (compare Franke et al. (2017)for white noise and Borghuis et al. (2013) for spots). The described diameter sizes measured with binary white noise usually match the dendritic field sizes of bipolar cells. Yet, the diameter sizes with spot stimuli are much larger than the dendritic fields. How to explain such discrepancy?

A possible explanation might be light scattering in the retina. The idea is that through light scattering the spot edges become fuzzy. The light scattering could originate from photoreceptors outer segments (Borges and Wilson, 1987) or from the other neurons if light first has to pass through the entire retina (Hare and Owen, 1990). Hare and Owen (1990) tested the role of light scattering inside the retina by comparing the receptive field sizes of bipolar cells when projecting the light directly on the photoreceptors (from above) vs. projecting the light first through the retina (from below). They did not observe a difference in the receptive field size and therefore concluded that light scattering is insignificant. Borges and Wilson (1987) studied the light scattering at the photoreceptor outer segments and found that light scattering at the photoreceptors is not a significant problem for receptive field measurements. Both studies explain their large receptive field sizes through internal coupling among bipolar cells. Because we see a clear differences in the diameter size measured with spots vs. binary white noise, it might well be that the binary white noise stimulus does not activate the coupling as strongly as the spots. The idea would be that the small squares of the binary white noise stimulus are not strong enough to influence the signals among neigbouring bipolar cells and therefore represent the dentritic field size of bipolar cells. The increasing spot stimulus on the other hand clearly activates with increasing size more and more bipolar cells and therefore reflects the excitatory receptive field of the bipolar cell beyond its dentritic tree.

To study these findings further, it would be interesting to experimentally block the gap junctions among the bipolar cells and measure the changes in receptive field size. A possible hypothesis is that the measured differences between binary white noise and spots is due to gap 
7. Discussion and Outlook

junctions and through the blocking of the coupling, the diameters measured with spots might become closer to the white-noise estimation. 


\section{References}

Aguilar, M., and Stiles, W. (1954). Saturation of the rod mechanism of the retina at high levels of stimulation. Optica Acta: International Journal of Optics 1, 59-65.

Applebury, M.L., Antoch, M.P., Baxter, L.C., Chun, L.L., Falk, J.D., Farhangfar, F., Kage, K., Krzystolik, M.G., Lyass, L.A., and Robbins, J.T. (2000). The murine cone photoreceptor: a single cone type expresses both $\mathrm{S}$ and $\mathrm{M}$ opsins with retinal spatial patterning. Neuron 27, 513-523.

Asari, H., and Meister, M. (2012). Divergence of visual channels in the inner retina. Nat Neurosci 15, 1581-1589.

Asari, H., and Meister, M. (2014). The projective field of retinal bipolar cells and its modulation by visual context. Neuron $81,641-652$.

Ashmore, J.F., and Falk, G. (1980). Responses of rod bipolar cells in the dark-adapted retina of the dogfish, Scyliorhinus canicula. J Physiol 300, 115-150.

Attwell, D., Mobbs, P., Tessier-Lavigne, M., and Wilson, M. (1987). Neurotransmitter-induced currents in retinal bipolar cells of the axolotl, Ambystoma mexicanum. J Physiol 387, 125-161.

Awatramani, G.B., and Slaughter, M.M. (2000). Origin of transient and sustained responses in ganglion cells of the retina. J Neurosci 20, 7087-7095.

Baccus, S.A. (2007). Timing and computation in inner retinal circuitry. Annu Rev Physiol 69, 271290.

Baccus, S.A., and Meister, M. (2002). Fast and slow contrast adaptation in retinal circuitry. Neuron 36, 909-919.

Baccus, S.A., Olveczky, B.P., Manu, M., and Meister, M. (2008). A retinal circuit that computes object motion. J Neurosci 28, 6807-6817.

Baden, T., Berens, P., Bethge, M., and Euler, T. (2013a). Spikes in mammalian bipolar cells support temporal layering of the inner retina. Curr Biol 23, 48-52.

Baden, T., Berens, P., Franke, K., Roman Roson, M., Bethge, M., and Euler, T. (2016). The functional diversity of retinal ganglion cells in the mouse. Nature 529, 345-350. 
Baden, T., Esposti, F., Nikolaev, A., and Lagnado, L. (2011). Spikes in retinal bipolar cells phaselock to visual stimuli with millisecond precision. Curr Biol 21, 1859-1869.

Baden, T., Euler, T., Weckstrom, M., and Lagnado, L. (2013b). Spikes and ribbon synapses in early vision. Trends Neurosci 36, 480-488.

Baden, T., Schubert, T., Chang, L., Wei, T., Zaichuk, M., Wissinger, B., and Euler, T. (2013c). A tale of two retinal domains: near-optimal sampling of achromatic contrasts in natural scenes through asymmetric photoreceptor distribution. Neuron 80, 1206-1217.

Barlow, H.B. (1953). Summation and inhibition in the frog's retina. J Physiol 119, 69-88.

Barlow, H.B., Levick, W.R., and Yoon, M. (1971). Responses to single quanta of light in retinal ganglion cells of the cat. Vision Res Suppl 3, 87-101.

Baylor, D.A., Fuortes, M.G., and O'Bryan, P.M. (1971). Receptive fields of cones in the retina of the turtle. J Physiol 214, 265-294.

Baylor, D.A., Hodgkin, A.L., and Lamb, T.D. (1974). The electrical response of turtle cones to flashes and steps of light. J Physiol 242, 685-727.

Berntson, A., and Taylor, W.R. (2000). Response characteristics and receptive field widths of onbipolar cells in the mouse retina. J Physiol 524 Pt 3, 879-889.

Betsch, B.Y., Einhauser, W., Kording, K.P., and Konig, P. (2004). The world from a cat's perspective--statistics of natural videos. Biol Cybern 90, 41-50.

Bolinger, D., and Gollisch, T. (2012). Closed-loop measurements of iso-response stimuli reveal dynamic nonlinear stimulus integration in the retina. Neuron 73, 333-346.

Borges, S., and Wilson, M. (1987). Structure of the receptive fields of bipolar cells in the salamander retina. J Neurophysiol 58, 1275-1291.

Borghuis, B.G., Looger, L.L., Tomita, S., and Demb, J.B. (2014). Kainate receptors mediate signaling in both transient and sustained OFF bipolar cell pathways in mouse retina. J Neurosci 34, 6128-6139.

Borghuis, B.G., Marvin, J.S., Looger, L.L., and Demb, J.B. (2013). Two-photon imaging of nonlinear glutamate release dynamics at bipolar cell synapses in the mouse retina. J Neurosci 33, 10972-10985.

Bortoff, A. (1964). Localization of slow potential responses in the Necturus retina. Vision Res 4, 627-635.

Bortoff, A., and Norton, A.L. (1965). Positive and negative potential responses associated with vertebrate photoreceptor cells. Nature 206, 626-627.

Boycott, B.B., and Wassle, H. (1974). The morphological types of ganglion cells of the domestic cat's retina. J Physiol 240, 397-419.

Brandstatter, J.H., Koulen, P., and Wassle, H. (1997). Selective synaptic distribution of kainate receptor subunits in the two plexiform layers of the rat retina. J Neurosci 17, 9298-9307. 
Brandstatter, J.H., Koulen, P., and Wassle, H. (1998). Diversity of glutamate receptors in the mammalian retina. Vision Res 38, 1385-1397.

Breuninger, T., Puller, C., Haverkamp, S., and Euler, T. (2011). Chromatic bipolar cell pathways in the mouse retina. J Neurosci 31, 6504-6517.

Burkhardt, D.A. (1974). Sensitization and centre-surround antagonism in Necturus retina. J Physiol 236, 593-610.

Burkhardt, D.A., Bartoletti, T.M., and Thoreson, W.B. (2011). Center/surround organization of retinal bipolar cells: High correlation of fundamental responses of center and surround to sinusoidal contrasts. Vis Neurosci 28, 183-192.

Burkhardt, D.A., and Fahey, P.K. (1998). Contrast enhancement and distributed encoding by bipolar cells in the retina. J Neurophysiol 80, 1070-1081.

Burkhardt, D.A., Fahey, P.K., and Sikora, M. (1998). Responses of ganglion cells to contrast steps in the light-adapted retina of the tiger salamander. Vis Neurosci 15, 219-229.

Burrone, J., and Lagnado, L. (1997). Electrical resonance and Ca2+ influx in the synaptic terminal of depolarizing bipolar cells from the goldfish retina. J Physiol 505 (Pt 3), 571-584.

Carcieri, S.M., Jacobs, A.L., and Nirenberg, S. (2003). Classification of retinal ganglion cells: a statistical approach. J Neurophysiol 90, 1704-1713.

Chapot, C.A., Euler, T., and Schubert, T. (2017). How do horizontal cells 'talk' to cone photoreceptors? Different levels of complexity at the cone-horizontal cell synapse. J Physiol 595, 5495-5506.

Chichilnisky, E.J. (2001). A simple white noise analysis of neuronal light responses. Network 12, 199-213.

Cleland, B.G., Dubin, M.W., and Levick, W.R. (1971). Sustained and transient neurones in the cat's retina and lateral geniculate nucleus. J Physiol 217, 473-496.

Cleland, B.G., and Levick, W.R. (1974). Properties of rarely encountered types of ganglion cells in the cat's retina and an overall classification. J Physiol 240, 457-492.

Cleland, B.G., Levick, W.R., and Sanderson, K.J. (1973). Properties of sustained and transient ganglion cells in the cat retina. J Physiol 228, 649-680.

Contini, M., and Raviola, E. (2003). GABAergic synapses made by a retinal dopaminergic neuron. Proc Natl Acad Sci U S A 100, 1358-1363.

Cook, P.B., and McReynolds, J.S. (1998). Lateral inhibition in the inner retina is important for spatial tuning of ganglion cells. Nat Neurosci 1, 714-719.

Coombs, J., van der List, D., Wang, G.Y., and Chalupa, L.M. (2006). Morphological properties of mouse retinal ganglion cells. Neuroscience 140, 123-136.

Crook, J.D., Peterson, B.B., Packer, O.S., Robinson, F.R., Troy, J.B., and Dacey, D.M. (2008). Ycell receptive field and collicular projection of parasol ganglion cells in macaque monkey retina. J Neurosci 28, 11277-11291. 
Dacey, D., Packer, O.S., Diller, L., Brainard, D., Peterson, B., and Lee, B. (2000). Center surround receptive field structure of cone bipolar cells in primate retina. Vision Res 40, 1801 1811.

Dacey, D.M., Lee, B.B., Stafford, D.K., Pokorny, J., and Smith, V.C. (1996). Horizontal cells of the primate retina: cone specificity without spectral opponency. Science 271, 656-659.

Dayan, P., and Abbott, L.F. (2001). Theoretical neuroscience: computational and mathematical modeling of neural systems (Cambridge, MA: MIT Press).

de Monasterio, F.M. (1978). Properties of concentrically organized X and Y ganglion cells of macaque retina. J Neurophysiol 41, 1394-1417.

de Vries, S.E., Baccus, S.A., and Meister, M. (2011). The projective field of a retinal amacrine cell. J Neurosci 31, 8595-8604.

Demb, J.B., Haarsma, L., Freed, M.A., and Sterling, P. (1999). Functional circuitry of the retinal ganglion cell's nonlinear receptive field. J Neurosci 19, 9756-9767.

Demb, J.B., and Singer, J.H. (2015). Functional Circuitry of the Retina. Annu Rev Vis Sci 1, 263289.

Demb, J.B., Zaghloul, K., Haarsma, L., and Sterling, P. (2001a). Bipolar cells contribute to nonlinear spatial summation in the brisk-transient $(\mathrm{Y})$ ganglion cell in mammalian retina. $\mathrm{J}$ Neurosci 21, 7447-7454.

Demb, J.B., Zaghloul, K., and Sterling, P. (2001b). Cellular basis for the response to second-order motion cues in Y retinal ganglion cells. Neuron 32, 711-721.

DeVries, S.H. (2000). Bipolar cells use kainate and AMPA receptors to filter visual information into separate channels. Neuron 28, 847-856.

DeVries, S.H., and Schwartz, E.A. (1999). Kainate receptors mediate synaptic transmission between cones and 'Off' bipolar cells in a mammalian retina. Nature 397, 157-160.

Dong, C.J., and Werblin, F.S. (1998). Temporal contrast enhancement via GABAC feedback at bipolar terminals in the tiger salamander retina. J Neurophysiol 79, 2171-2180.

Donner, K., and Gronholm, M.L. (1984). Center and surround excitation in the receptive fields of frog retinal ganglion cells. Vision Res 24, 1807-1819.

Dreosti, E., Esposti, F., Baden, T., and Lagnado, L. (2011). In vivo evidence that retinal bipolar cells generate spikes modulated by light. Nat Neurosci 14, 951-952.

Dumitrescu, O.N., Protti, D.A., Majumdar, S., Zeilhofer, H.U., and Wassle, H. (2006). Ionotropic glutamate receptors of amacrine cells of the mouse retina. Vis Neurosci 23, 79-90.

Dunn, F.A., Lankheet, M.J., and Rieke, F. (2007). Light adaptation in cone vision involves switching between receptor and post-receptor sites. Nature 449, 603-606.

Dunn, F.A., and Rieke, F. (2008). Single-photon absorptions evoke synaptic depression in the retina to extend the operational range of rod vision. Neuron 57, 894-904. 
Eggers, E.D., and Lukasiewicz, P.D. (2010). Interneuron circuits tune inhibition in retinal bipolar cells. J Neurophysiol 103, 25-37.

Eggers, E.D., and Lukasiewicz, P.D. (2011). Multiple pathways of inhibition shape bipolar cell responses in the retina. Vis Neurosci 28, 95-108.

Enroth-Cugell, C., and Robson, J.G. (1966). The contrast sensitivity of retinal ganglion cells of the cat. J Physiol 187, 517-552.

Enroth-Cugell, C., and Robson, J.G. (1984). Functional characteristics and diversity of cat retinal ganglion cells. Basic characteristics and quantitative description. Investigative ophthalmology \& visual science $25,250-267$.

Euler, T., and Denk, W. (2001). Dendritic processing. Curr Opin Neurobiol 11, 415-422.

Euler, T., Haverkamp, S., Schubert, T., and Baden, T. (2014). Retinal bipolar cells: elementary building blocks of vision. Nat Rev Neurosci 15, 507-519.

Euler, T., and Masland, R.H. (2000). Light-evoked responses of bipolar cells in a mammalian retina. J Neurophysiol 83, 1817-1829.

Fahey, P.K., and Burkhardt, D.A. (2003). Center-surround organization in bipolar cells: symmetry for opposing contrasts. Vis Neurosci 20, 1-10.

Fain, G.L., and Dowling, J.E. (1973). Intracellular recordings from single rods and cones in the mudpuppy retina. Science 180,1178-1181.

Famiglietti, E., and Kolb, H. (1976). Structural basis for ON-and OFF-center responses in retinal ganglion cells. Science 194, 193-195.

Farrow, K., Teixeira, M., Szikra, T., Viney, T.J., Balint, K., Yonehara, K., and Roska, B. (2013). Ambient illumination toggles a neuronal circuit switch in the retina and visual perception at cone threshold. Neuron 78, 325-338.

Field, G.D., and Chichilnisky, E.J. (2007). Information processing in the primate retina: circuitry and coding. Annu Rev Neurosci 30, 1-30.

Field, G.D., and Rieke, F. (2002). Nonlinear signal transfer from mouse rods to bipolar cells and implications for visual sensitivity. Neuron 34, 773-785.

Franke, K., and Baden, T. (2017). General features of inhibition in the inner retina. J Physiol 595, 5507-5515.

Franke, K., Berens, P., Schubert, T., Bethge, M., Euler, T., and Baden, T. (2017). Inhibition decorrelates visual feature representations in the inner retina. Nature 542, 439-444.

Freed, M.A. (2000). Parallel cone bipolar pathways to a ganglion cell use different rates and amplitudes of quantal excitation. J Neurosci 20, 3956-3963.

Freed, M.A., Pflug, R., Kolb, H., and Nelson, R. (1996). ON-OFF amacrine cells in cat retina. J Comp Neurol 364, 556-566. 
Freeman, J., Field, G.D., Li, P.H., Greschner, M., Gunning, D.E., Mathieson, K., Sher, A., Litke, A.M., Paninski, L., Simoncelli, E.P., and Chichilnisky, E.J. (2015). Mapping nonlinear receptive field structure in primate retina at single cone resolution. Elife 4, e05241.

Gauthier, J.L., Field, G.D., Sher, A., Greschner, M., Shlens, J., Litke, A.M., and Chichilnisky, E.J. (2009). Receptive fields in primate retina are coordinated to sample visual space more uniformly. PLoS Biol 7, e1000063.

Ghosh, K.K., Bujan, S., Haverkamp, S., Feigenspan, A., and Wassle, H. (2004). Types of bipolar cells in the mouse retina. J Comp Neurol 469, 70-82.

Gold, C., Henze, D.A., Koch, C., and Buzsaki, G. (2006). On the origin of the extracellular action potential waveform: A modeling study. J Neurophysiol 95, 3113-3128.

Gollisch, T. (2013). Features and functions of nonlinear spatial integration by retinal ganglion cells. J Physiol Paris 107, 338-348.

Gollisch, T., and Meister, M. (2010). Eye smarter than scientists believed: neural computations in circuits of the retina. Neuron 65, 150-164.

Hare, W.A., Lowe, J.S., and Owen, G. (1986). Morphology of physiologically identified bipolar cells in the retina of the tiger salamander, Ambystoma tigrinum. J Comp Neurol 252, 130-138.

Hare, W.A., and Owen, W.G. (1990). Spatial organization of the bipolar cell's receptive field in the retina of the tiger salamander. J Physiol 421, 223-245.

Hare, W.A., and Owen, W.G. (1996). Receptive field of the retinal bipolar cell: a pharmacological study in the tiger salamander. J Neurophysiol 76, 2005-2019.

Hartline, H.K. (1938). The response of single optic nerve fibers of the vertebrate eye to illumination of the retina. American Journal of Physiology-Legacy Content 121, 400-415.

Haverkamp, S., Specht, D., Majumdar, S., Zaidi, N.F., Brandstatter, J.H., Wasco, W., Wassle, H., and Tom Dieck, S. (2008). Type 4 OFF cone bipolar cells of the mouse retina express calsenilin and contact cones as well as rods. J Comp Neurol 507, 1087-1101.

Haverkamp, S., Wassle, H., Duebel, J., Kuner, T., Augustine, G.J., Feng, G., and Euler, T. (2005). The primordial, blue-cone color system of the mouse retina. J Neurosci 25, 5438-5445.

He, S., and Macleod, D.I. (1998). Contrast-modulation flicker: dynamics and spatial resolution of the light adaptation process. Vision Res 38, 985-1000.

Hecht, S., Shlaer, S., and Pirenne, M.H. (1941). Energy at the Threshold of Vision. Science 93, 585-587.

Heine, W.F., and Passaglia, C.L. (2011). Spatial receptive field properties of rat retinal ganglion cells. Vis Neurosci 28, 403-417.

Heitman, A., Brackbill, N., Greschner, M., Sher, A., Litke, A.M., and Chichilnisky, E. (2016). Testing pseudo-linear models of responses to natural scenes in primate retina. bioRxiv, 045336 .

Helmstaedter, M., Briggman, K.L., Turaga, S.C., Jain, V., Seung, H.S., and Denk, W. (2013).

Connectomic reconstruction of the inner plexiform layer in the mouse retina. Nature 500, 168174. 
Hochstein, S., and Shapley, R.M. (1976a). Linear and nonlinear spatial subunits in Y cat retinal ganglion cells. J Physiol 262, 265-284.

Hochstein, S., and Shapley, R.M. (1976b). Quantitative analysis of retinal ganglion cell classifications. J Physiol 262, 237-264.

Hoon, M., Sinha, R., Okawa, H., Suzuki, S.C., Hirano, A.A., Brecha, N., Rieke, F., and Wong, R.O. (2015). Neurotransmission plays contrasting roles in the maturation of inhibitory synapses on axons and dendrites of retinal bipolar cells. Proc Natl Acad Sci U S A 112, 12840-12845.

Hou, M., Duan, L., and Slaughter, M.M. (2008). Synaptic inhibition by glycine acting at a metabotropic receptor in tiger salamander retina. J Physiol 586, 2913-2926.

Ingram, N.T., Sampath, A.P., and Fain, G.L. (2016). Why are rods more sensitive than cones? J Physiol 594, 5415-5426.

Jacoby, J., and Schwartz, G.W. (2017). Three Small-Receptive-Field Ganglion Cells in the Mouse Retina Are Distinctly Tuned to Size, Speed, and Object Motion. J Neurosci 37, 610-625.

Jeon, C.J., Strettoi, E., and Masland, R.H. (1998). The major cell populations of the mouse retina. J Neurosci 18, 8936-8946.

Johnson, K.P., Zhao, L., and Kerschensteiner, D. (2018). A Pixel-Encoder Retinal Ganglion Cell with Spatially Offset Excitatory and Inhibitory Receptive Fields. Cell Rep 22, 1462-1472.

Kaneko, A. (1970). Physiological and morphological identification of horizontal, bipolar and amacrine cells in goldfish retina. J Physiol 207, 623-633.

Katz, M.L., Viney, T.J., and Nikolic, K. (2016). Receptive Field Vectors of Genetically-Identified Retinal Ganglion Cells Reveal Cell-Type-Dependent Visual Functions. PLoS One 11, e0147738.

Kawai, F., Horiguchi, M., Suzuki, H., and Miyachi, E. (2001). $\mathrm{Na}(+)$ action potentials in human photoreceptors. Neuron 30, 451-458.

Kayser, C., Salazar, R.F., and Konig, P. (2003). Responses to natural scenes in cat V1. J

Neurophysiol 90, 1910-1920.

Kemmler, R., Schultz, K., Dedek, K., Euler, T., and Schubert, T. (2014). Differential regulation of cone calcium signals by different horizontal cell feedback mechanisms in the mouse retina. J Neurosci 34, 11826-11843.

Klumpp, D.J., Song, E.J., Ito, S., Sheng, M.H., Jan, L.Y., and Pinto, L.H. (1995). The Shaker-like potassium channels of the mouse rod bipolar cell and their contributions to the membrane current. J Neurosci 15, 5004-5013.

Kolb, H., Linberg, K.A., and Fisher, S.K. (1992). Neurons of the human retina: a Golgi study. J Comp Neurol 318, 147-187.

Kong, J.H., Fish, D.R., Rockhill, R.L., and Masland, R.H. (2005). Diversity of ganglion cells in the mouse retina: unsupervised morphological classification and its limits. J Comp Neurol 489, 293310.

Krieger, B., Qiao, M., Rousso, D.L., Sanes, J.R., and Meister, M. (2017). Four alpha ganglion cell types in mouse retina: Function, structure, and molecular signatures. PLoS One 12, e0180091. 
Krishnamoorthy, V., Weick, M., and Gollisch, T. (2017). Sensitivity to image recurrence across eye-movement-like image transitions through local serial inhibition in the retina. Elife 6, e22431.

Kuffler, S.W. (1953). Discharge patterns and functional organization of mammalian retina. J Neurophysiol 16, 37-68.

Kuhn, N.K., and Gollisch, T. (2016). Joint Encoding of Object Motion and Motion Direction in the Salamander Retina. J Neurosci 36, 12203-12216.

Lee, S., Chen, L., Chen, M., Ye, M., Seal, R.P., and Zhou, Z.J. (2014). An unconventional glutamatergic circuit in the retina formed by vGluT3 amacrine cells. Neuron 84, 708-715.

Lettvin, J.Y., Maturana, H.R., McCulloch, W.S., and Pitts, W.H. (1959). What the frog's eye tells the frog's brain. Proceedings of the IRE 47, 1940-1951.

Lewicki, M.S. (1998). A review of methods for spike sorting: the detection and classification of neural action potentials. Network 9, R53-78.

Li, T., Wu, S.M., Lam, D.M., and Watt, C.B. (1990). Localization of classical neurotransmitters in interneurons of the larval tiger salamander retina. Invest Ophthalmol Vis Sci 31, 262-271.

Liang, Z., and Freed, M.A. (2010). The ON pathway rectifies the OFF pathway of the mammalian retina. J Neurosci 30, 5533-5543.

Lindstrom, S.H., Ryan, D.G., Shi, J., and DeVries, S.H. (2014). Kainate receptor subunit diversity underlying response diversity in retinal off bipolar cells. J Physiol 592, 1457-1477.

Liu, J.K., Schreyer, H.M., Onken, A., Rozenblit, F., Khani, M.H., Krishnamoorthy, V., Panzeri, S., and Gollisch, T. (2017). Inference of neuronal functional circuitry with spike-triggered nonnegative matrix factorization. Nat Commun $8,149$.

Maheswaranathan, N., Baccus, S.A., and Ganguli, S. (2017). Inferring hidden structure in multilayered neural circuits. bioRxiv, 120956.

Mangel, S.C. (1991). Analysis of the horizontal cell contribution to the receptive field surround of ganglion cells in the rabbit retina. J Physiol 442, 211-234.

Mangel, S.C., and Miller, R.F. (1987). Horizontal cells contribute to the receptive field surround of ganglion cells in the rabbit retina. Brain Res 414, 182-186.

Mani, A., and Schwartz, G.W. (2017). Circuit Mechanisms of a Retinal Ganglion Cell with Stimulus-Dependent Response Latency and Activation Beyond Its Dendrites. Curr Biol 27, 471482.

Manookin, M.B., Beaudoin, D.L., Ernst, Z.R., Flagel, L.J., and Demb, J.B. (2008). Disinhibition combines with excitation to extend the operating range of the OFF visual pathway in daylight. J Neurosci 28, 4136-4150.

Mante, V., Bonin, V., and Carandini, M. (2008). Functional mechanisms shaping lateral geniculate responses to artificial and natural stimuli. Neuron 58, 625-638.

Mao, B.Q., MacLeish, P.R., and Victor, J.D. (1998). The intrinsic dynamics of retinal bipolar cells isolated from tiger salamander. Visual neuroscience 15, 425-438. 
Maricq, A.V., and Korenbrot, J.I. (1988). Calcium and calcium-dependent chloride currents generate action potentials in solitary cone photoreceptors. Neuron 1, 503-515.

Masland, R.H. (2001). The fundamental plan of the retina. Nat Neurosci 4, 877-886.

Masland, R.H. (2004). Neuronal cell types. Curr Biol 14, R497-500.

Masland, R.H. (2012a). The neuronal organization of the retina. Neuron 76, 266-280.

Masland, R.H. (2012b). The tasks of amacrine cells. Vis Neurosci 29, 3-9.

Masland, R.H., and Mills, J.W. (1979). Autoradiographic identification of acetylcholine in the rabbit retina. J Cell Biol 83, 159-178.

Massey, S.C., and Miller, R.F. (1988). Glutamate receptors of ganglion cells in the rabbit retina: evidence for glutamate as a bipolar cell transmitter. J Physiol 405, 635-655.

Mataruga, A., Kremmer, E., and Muller, F. (2007). Type 3a and type 3b OFF cone bipolar cells provide for the alternative rod pathway in the mouse retina. J Comp Neurol 502, 1123-1137.

Matsui, K., Hosoi, N., and Tachibana, M. (1998). Excitatory synaptic transmission in the inner retina: paired recordings of bipolar cells and neurons of the ganglion cell layer. J Neurosci 18 , $4500-4510$.

Menger, N., Pow, D.V., and Wassle, H. (1998). Glycinergic amacrine cells of the rat retina. J Comp Neurol 401, 34-46.

Molnar, A., Hsueh, H.A., Roska, B., and Werblin, F.S. (2009). Crossover inhibition in the retina: circuitry that compensates for nonlinear rectifying synaptic transmission. J Comput Neurosci 27, 569-590.

Morigiwa, K., and Vardi, N. (1999). Differential expression of ionotropic glutamate receptor subunits in the outer retina. J Comp Neurol 405, 173-184.

Munch, T.A., da Silveira, R.A., Siegert, S., Viney, T.J., Awatramani, G.B., and Roska, B. (2009). Approach sensitivity in the retina processed by a multifunctional neural circuit. Nat Neurosci 12, 1308-1316.

Naka, K.I., Itoh, M.A., and Chappell, R.L. (1987). Dynamics of turtle cones. J Gen Physiol 89, 321-337.

Nawy, S. (2000). Regulation of the on bipolar cell mGluR6 pathway by Ca2+. J Neurosci 20, 4471-4479.

Nirenberg, S., and Meister, M. (1997). The light response of retinal ganglion cells is truncated by a displaced amacrine circuit. Neuron 18, 637-650.

Nomura, A., Shigemoto, R., Nakamura, Y., Okamoto, N., Mizuno, N., and Nakanishi, S. (1994). Developmentally regulated postsynaptic localization of a metabotropic glutamate receptor in rat rod bipolar cells. Cell 77, 361-369.

Ölveczky, B.P., Baccus, S.A., and Meister, M. (2003). Segregation of object and background motion in the retina. Nature 423, 401-408. 
Palmer, M.J. (2010). Characterisation of bipolar cell synaptic transmission in goldfish retina using paired recordings. J Physiol 588, 1489-1498.

Pang, J.J., Gao, F., and Wu, S.M. (2004). Stratum-by-stratum projection of light response attributes by retinal bipolar cells of Ambystoma. J Physiol 558, 249-262.

Peichl, L., and Gonzalez-Soriano, J. (1994). Morphological types of horizontal cell in rodent retinae: a comparison of rat, mouse, gerbil, and guinea pig. Vis Neurosci 11, 501-517.

Peng, Y.W., Blackstone, C.D., Huganir, R.L., and Yau, K.W. (1995). Distribution of glutamate receptor subtypes in the vertebrate retina. Neuroscience 66, 483-497.

Petrusca, D., Grivich, M.I., Sher, A., Field, G.D., Gauthier, J.L., Greschner, M., Shlens, J., Chichilnisky, E.J., and Litke, A.M. (2007). Identification and characterization of a Y-like primate retinal ganglion cell type. J Neurosci 27, 11019-11027.

Pillow, J.W., Paninski, L., Uzzell, V.J., Simoncelli, E.P., and Chichilnisky, E.J. (2005). Prediction and decoding of retinal ganglion cell responses with a probabilistic spiking model. J Neurosci 25, 11003-11013.

Pillow, J.W., Shlens, J., Paninski, L., Sher, A., Litke, A.M., Chichilnisky, E.J., and Simoncelli, E.P. (2008). Spatio-temporal correlations and visual signalling in a complete neuronal population. Nature 454, 995-999.

Pourcho, R.G., and Goebel, D.J. (1983). Neuronal subpopulations in cat retina which accumulate the GABA agonist, $(3 \mathrm{H})$ muscimol: a combined Golgi and autoradiographic study. J Comp Neurol 219, 25-35.

Pouzat, C., Mazor, O., and Laurent, G. (2002). Using noise signature to optimize spike-sorting and to assess neuronal classification quality. J Neurosci Methods 122, 43-57.

Puller, C., Haverkamp, S., Neitz, M., and Neitz, J. (2014). Synaptic elements for GABAergic feedforward signaling between HII horizontal cells and blue cone bipolar cells are enriched beneath primate S-cones. PLoS One 9, e88963.

Puller, C., Ivanova, E., Euler, T., Haverkamp, S., and Schubert, T. (2013). OFF bipolar cells express distinct types of dendritic glutamate receptors in the mouse retina. Neuroscience 243, 136-148.

Purgert, R.J., and Lukasiewicz, P.D. (2015). Differential encoding of spatial information among retinal on cone bipolar cells. J Neurophysiol 114, 1757-1772.

Qin, P., and Pourcho, R.G. (1996). Distribution of AMPA-selective glutamate receptor subunits in the cat retina. Brain Res 710, 303-307.

Quiroga, R.Q., Nadasdy, Z., and Ben-Shaul, Y. (2004). Unsupervised spike detection and sorting with wavelets and superparamagnetic clustering. Neural Comput 16, 1661-1687.

Reinhard, K., Tikidji-Hamburyan, A., Seitter, H., Idrees, S., Mutter, M., Benkner, B., and Munch, T.A. (2014). Step-by-step instructions for retina recordings with perforated multi electrode arrays. PLoS One 9, e106148.

Rieke, F. (2001). Temporal contrast adaptation in salamander bipolar cells. J Neurosci 21, 94459454. 
Rodieck, R.W. (1998). The first steps in seeing (Sunderland, Mass.: Sinauer Associates).

Roska, B., and Meister, M. (2014). The retina dissects the visual scene into distinct features. In The New Visual Neurosciences, J.S. Werner, and L.M. Chalupa, eds. (Cambridge, MA: MIT Press), pp. 163-182.

Roska, B., Nemeth, E., Orzo, L., and Werblin, F.S. (2000). Three levels of lateral inhibition: A space-time study of the retina of the tiger salamander. J Neurosci 20, 1941-1951.

Roska, B., and Werblin, F. (2001). Vertical interactions across ten parallel, stacked representations in the mammalian retina. Nature 410, 583-587.

Roth, G. (1987). Visual Behavior in Salamanders (Germany: Springer-Verlag).

Saito, H.A. (1983). Morphology of physiologically identified X-, Y-, and W-type retinal ganglion cells of the cat. J Comp Neurol 221, 279-288.

Sakai, H.M., and Naka, K. (1987a). Signal transmission in the catfish retina. IV. Transmission to ganglion cells. J Neurophysiol 58, 1307-1328.

Sakai, H.M., and Naka, K. (1987b). Signal transmission in the catfish retina. V. Sensitivity and circuit. J Neurophysiol 58, 1329-1350.

Sakai, H.M., Wang, J.L., and Naka, K. (1995). Contrast gain control in the lower vertebrate retinas. J Gen Physiol 105, 815-835.

Satoh, H., Aoki, K., Watanabe, S.I., and Kaneko, A. (1998). L-type calcium channels in the axon terminal of mouse bipolar cells. Neuroreport 9, 2161-2165.

Schultze, M. (1866). Zur anatomie und physiologie der retina. Archiv für mikroskopische Anatomie 2, 175-286.

Schwartz, G., and Rieke, F. (2011). Perspectives on: information and coding in mammalian sensory physiology: nonlinear spatial encoding by retinal ganglion cells: when $1+1$ not equal 2 . J Gen Physiol 138, 283-290.

Schwartz, G.W., Okawa, H., Dunn, F.A., Morgan, J.L., Kerschensteiner, D., Wong, R.O., and Rieke, F. (2012). The spatial structure of a nonlinear receptive field. Nat Neurosci 15, 1572-1580.

Segev, R., Puchalla, J., and Berry, M.J., 2nd (2006). Functional organization of ganglion cells in the salamander retina. J Neurophysiol 95, 2277-2292.

Shapley, R. (2009). Linear and nonlinear systems analysis of the visual system: why does it seem so linear? A review dedicated to the memory of Henk Spekreijse. Vision Res 49, 907-921.

Sherry, D.M., Bui, D.D., and Degrip, W.J. (1998). Identification and distribution of photoreceptor subtypes in the neotenic tiger salamander retina. Vis Neurosci 15, 1175-1187.

Shields, C.R., Tran, M.N., Wong, R.O., and Lukasiewicz, P.D. (2000). Distinct ionotropic GABA receptors mediate presynaptic and postsynaptic inhibition in retinal bipolar cells. J Neurosci 20, 2673-2682.

Shiells, R.A., and Falk, G. (1999). A rise in intracellular Ca2+ underlies light adaptation in dogfish retinal 'on' bipolar cells. J Physiol 514 ( Pt 2), 343-350. 
Simmons, P.J., and Young, D. (1999). Nerve cells and animal behaviour (Cambridge (UK):

Cambridge University Press).

Sinex, D.G., Burdette, L.J., and Pearlman, A.L. (1979). A psychophysical investigation of spatial vision in the normal and reeler mutant mouse. Vision Res 19, 853-857.

Skrzypek, J., and Werblin, F. (1983). Lateral interactions in absence of feedback to cones. J Neurophysiol 49, 1007-1016.

Stafford, D.K., and Dacey, D.M. (1997). Physiology of the A1 amacrine: a spiking, axon-bearing interneuron of the macaque monkey retina. Vis Neurosci 14, 507-522.

Sterling, P., and Demb, J.B. (2004). Retina. In The synaptic organization of the brain, G.M. Shepherd, ed. (New York, USA: Oxford university press), pp. 217-269.

Stone, C., and Pinto, L.H. (1993). Response properties of ganglion cells in the isolated mouse retina. Vis Neurosci 10, 31-39.

Stone, J., and Hoffmann, K.P. (1972). Very slow-conducting ganglion cells in the cat's retina: a major, new functional type? Brain Res 43, 610-616.

Sumbul, U., Song, S., McCulloch, K., Becker, M., Lin, B., Sanes, J.R., Masland, R.H., and Seung, H.S. (2014). A genetic and computational approach to structurally classify neuronal types. Nat Commun 5, 3512 .

Swaroop, A., Kim, D., and Forrest, D. (2010). Transcriptional regulation of photoreceptor development and homeostasis in the mammalian retina. Nature reviews. Neuroscience 11, 563576.

Szikra, T., Trenholm, S., Drinnenberg, A., Juttner, J., Raics, Z., Farrow, K., Biel, M., Awatramani, G., Clark, D.A., Sahel, J.A., et al. (2014). Rods in daylight act as relay cells for cone-driven horizontal cell-mediated surround inhibition. Nat Neurosci 17, 1728-1735.

Thibos, L.N., and Werblin, F.S. (1978). The response properties of the steady antagonistic surround in the mudpuppy retina. J Physiol 278, 79-99.

Thoreson, W.B., and Mangel, S.C. (2012). Lateral interactions in the outer retina. Prog Retin Eye Res 31, 407-441.

Thoreson, W.B., Tranchina, D., and Witkovsky, P. (2003). Kinetics of synaptic transfer from rods and cones to horizontal cells in the salamander retina. Neuroscience 122, 785-798.

Tikidji-Hamburyan, A., Reinhard, K., Seitter, H., Hovhannisyan, A., Procyk, C.A., Allen, A.E., Schenk, M., Lucas, R.J., and Munch, T.A. (2015). Retinal output changes qualitatively with every change in ambient illuminance. Nat Neurosci 18, 66-74.

Tikidji-Hamburyan, A., Reinhard, K., Storchi, R., Dietter, J., Seitter, H., Davis, K.E., Idrees, S., Mutter, M., Walmsley, L., Bedford, R.A., et al. (2017). Rods progressively escape saturation to drive visual responses in daylight conditions. Nat Commun 8, 1813.

Toris, C.B., Eiesland, J.L., and Miller, R.F. (1995). Morphology of ganglion cells in the neotenous tiger salamander retina. J Comp Neurol 352, 535-559. 
Toyoda, J. (1974). Frequency characteristics of retinal neurons in the carp. J Gen Physiol 63, 214234.

Troy, J.B., Schweitzer-Tong, D.E., and Enroth-Cugell, C. (1995). Receptive-field properties of Q retinal ganglion cells of the cat. Vis Neurosci 12, 285-300.

Tsukamoto, Y., and Omi, N. (2017). Classification of Mouse Retinal Bipolar Cells: Type-Specific Connectivity with Special Reference to Rod-Driven AII Amacrine Pathways. Front Neuroanat $11,92$.

Turner, M.H., and Rieke, F. (2016). Synaptic Rectification Controls Nonlinear Spatial Integration of Natural Visual Inputs. Neuron 90, 1257-1271.

Umino, Y., Solessio, E., and Barlow, R.B. (2008). Speed, spatial, and temporal tuning of rod and cone vision in mouse. J Neurosci 28, 189-198.

Van den Bergh, G., Zhang, B., Arckens, L., and Chino, Y.M. (2010). Receptive-field properties of V1 and V2 neurons in mice and macaque monkeys. J Comp Neurol 518, 2051-2070.

van Rossum, M.C., and Smith, R.G. (1998). Noise removal at the rod synapse of mammalian retina. Vis Neurosci 15, 809-821.

Veleri, S., Lazar, C.H., Chang, B., Sieving, P.A., Banin, E., and Swaroop, A. (2015). Biology and therapy of inherited retinal degenerative disease: insights from mouse models. Dis Model Mech 8 , 109-129.

Victor, J.D., and Shapley, R.M. (1979). The nonlinear pathway of Y ganglion cells in the cat retina. J Gen Physiol 74, 671-689.

Victor, J.D., Shapley, R.M., and Knight, B.W. (1977). Nonlinear analysis of cat retinal ganglion cells in the frequency domain. Proc Natl Acad Sci U S A 74, 3068-3072.

Volgyi, B., Chheda, S., and Bloomfield, S.A. (2009). Tracer coupling patterns of the ganglion cell subtypes in the mouse retina. J Comp Neurol 512, 664-687.

Wang, J., Jacoby, R., and Wu, S.M. (2016). Physiological and morphological characterization of ganglion cells in the salamander retina. Vision Res 119, 60-72.

Wassle, H. (2004). Parallel processing in the mammalian retina. Nat Rev Neurosci 5, 747-757.

Wassle, H., and Boycott, B.B. (1991). Functional architecture of the mammalian retina. Physiol Rev 71, 447-480.

Wassle, H., Puller, C., Muller, F., and Haverkamp, S. (2009). Cone contacts, mosaics, and territories of bipolar cells in the mouse retina. J Neurosci 29, 106-117.

Werblin, F.S. (2010). Six different roles for crossover inhibition in the retina: correcting the nonlinearities of synaptic transmission. Vis Neurosci 27, 1-8.

Werblin, F.S., and Dowling, J.E. (1969). Organization of the retina of the mudpuppy, Necturus maculosus. II. Intracellular recording. J Neurophysiol 32, 339-355. 
Witkovsky, P., Schmitz, Y., Akopian, A., Krizaj, D., and Tranchina, D. (1997). Gain of rod to horizontal cell synaptic transfer: relation to glutamate release and a dihydropyridine-sensitive calcium current. J Neurosci 17, 7297-7306.

Wu, S.M., Gao, F., and Maple, B.R. (2000). Functional architecture of synapses in the inner retina: segregation of visual signals by stratification of bipolar cell axon terminals. J Neurosci 20, $4462-4470$.

Yang, C.Y., Lukasiewicz, P., Maguire, G., Werblin, F.S., and Yazulla, S. (1991). Amacrine cells in the tiger salamander retina: morphology, physiology, and neurotransmitter identification. J Comp Neurol 312, 19-32.

Yang, X.L. (2004). Characterization of receptors for glutamate and GABA in retinal neurons. Prog Neurobiol 73, 127-150.

Yang, X.L., and Wu, S.M. (1991). Feedforward lateral inhibition in retinal bipolar cells: inputoutput relation of the horizontal cell-depolarizing bipolar cell synapse. Proc Natl Acad Sci U S A $88,3310-3313$.

Yazulla, S., and Studholme, K.M. (1998). Differential distribution of Shaker-like and Shab-like $\mathrm{K}+$-channel subunits in goldfish retina and retinal bipolar cells. J Comp Neurol 396, 131-140.

Yu, Y.C., Satoh, H., Wu, S.M., and Marshak, D.W. (2009). Histamine enhances voltage-gated potassium currents of ON bipolar cells in macaque retina. Invest Ophthalmol Vis Sci 50, 959965.

Zaghloul, K.A., Boahen, K., and Demb, J.B. (2003). Different circuits for ON and OFF retinal ganglion cells cause different contrast sensitivities. J Neurosci 23, 2645-2654.

Zaghloul, K.A., Boahen, K., and Demb, J.B. (2005). Contrast adaptation in subthreshold and spiking responses of mammalian Y-type retinal ganglion cells. J Neurosci 25, 860-868.

Zaghloul, K.A., Manookin, M.B., Borghuis, B.G., Boahen, K., and Demb, J.B. (2007). Functional circuitry for peripheral suppression in Mammalian Y-type retinal ganglion cells. J Neurophysiol 97, 4327-4340.

Zenisek, D., Henry, D., Studholme, K., Yazulla, S., and Matthews, G. (2001). Voltage-dependent sodium channels are expressed in nonspiking retinal bipolar neurons. J Neurosci 21, 4543-4550.

Zhang, A.J., and Wu, S.M. (2009). Receptive fields of retinal bipolar cells are mediated by heterogeneous synaptic circuitry. J Neurosci 29, 789-797.

Zhang, A.J., Zhang, J., and Wu, S.M. (2006). Electrical coupling, receptive fields, and relative $\mathrm{rod} /$ cone inputs of horizontal cells in the tiger salamander retina. J Comp Neurol 499, 422-431.

Zhang, C., and McCall, M.A. (2012). Receptor targets of amacrine cells. Vis Neurosci 29, 11-29.

Zhang, Y.F., Kim, I.J., Sanes, J.R., and Meister, M. (2012). The most numerous ganglion cell type of the mouse retina is a selective feature detector. P Natl Acad Sci USA 109, E2391-E2398.

Zucker, C., and Yazulla, S. (1982). Localization of synaptic and nonsynaptic nicotinicacetylcholine receptors in the goldfish retina. J Comp Neurol 204, 188-195. 